Portland State University

PDXScholar

Fall 12-3-2019

\title{
The Process of Establishing Theoretically Grounded Measures of Science and Chemistry Identity for Use in Undergraduate Chemistry Courses
}

Kathryn Nicole Hosbein

Portland State University

Follow this and additional works at: https://pdxscholar.library.pdx.edu/open_access_etds

Part of the Chemistry Commons

Let us know how access to this document benefits you.

\section{Recommended Citation}

Hosbein, Kathryn Nicole, "The Process of Establishing Theoretically Grounded Measures of Science and Chemistry Identity for Use in Undergraduate Chemistry Courses" (2019). Dissertations and Theses. Paper 5352.

https://doi.org/10.15760/etd.7225

This Dissertation is brought to you for free and open access. It has been accepted for inclusion in Dissertations and Theses by an authorized administrator of PDXScholar. Please contact us if we can make this document more accessible: pdxscholar@pdx.edu. 
The Process of Establishing Theoretically Grounded Measures of Science and Chemistry Identity for Use in Undergraduate Chemistry Courses

by

Kathryn Nicole Hosbein

A dissertation submitted in partial fulfillment of the requirements for the degree of

Doctor of Philosophy

in

Chemistry

\section{Dissertation Committee: \\ Jack Barbera, Chair \\ Dean B. Atkinson \\ Erin E. Shortlidge \\ Jason T. Newsom}

Portland State University

2019 
(C) 2019 Kathryn Nicole Hosbein 


\begin{abstract}
Identity has been theorized to aid in student persistence toward science, engineering, technology, and math (STEM) degrees. However, before we can explore the impacts of learning environments on identity, and subsequently persistence, robust measures that can aid in understanding how identity is fostered need to be available. This research study aims to create measures of identity by expanding an existing physics identity framework and contextualizing it to science and chemistry identities.

Development of the measures was carried out through two distinct phases that provided evidence for reliability and multiple aspects of validity.
\end{abstract}

The first phase of the study used qualitative methods to build upon an existing physics identity framework in order to support content validity for the new measures. Semi-structured interviews were performed with nine students from Portland State University using questions built from the physics identity framework and contextualized to science and chemistry. Thematic analysis was subsequently used to define themes that occurred throughout the interviews. The final themes were then aligned with theoretically supported constructs to build a novel framework for science and chemistry identities that included the constructs of mindset, situational interest, verbal persuasion, vicarious experiences, and mastery experiences.

The second phase of the study built upon phase one by utilizing quantitative methods to support response process, structural, and relational validity as well as reliability of the novel measures. This phase continued to build upon the physics identity framework by mirroring previous quantitative analyses with the constructs of situational 
interest, verbal persuasion, and mastery experiences. Surveys measuring these constructs, using both science and chemistry identity wording were distributed to students at five US institutions enrolled in either general $(n=341)$ or organic chemistry $(n=226)$ at both the beginning and end of the courses. Response process validity was established by performing cognitive interviews with a subset of the sample from one institution $(n=8)$. The structural validity of the measures was analyzed using confirmatory factor analysis (CFA) within each course for both wording types at each time point. Singleadministration reliability was estimated by omega. Relational validity was supported by mirroring relations between the physics identity constructs with situational interest, verbal persuasion, and mastery experiences through structural equation modeling (SEM). Issues with the science wording was discussed. A final structural model for chemistry was described and parameters within general and organic chemistry were discussed. Key findings for this final model showed that verbal persuasion and situational interest are directly related to chemistry identity while mastery experiences is indirectly related to chemistry identity through verbal persuasion and situational interest.

By creating a robust measure of chemistry identity and understanding the relations among the constructs involved in identity formation, researchers can now implement interventions to target relevant aspects of chemistry identity and measure the impact. While a final measure of science identity was not presented due to complications with the science-worded version of the measure, valuable implications were drawn from phase one using the theoretically supported constructs that represented both science and chemistry identity. Specifically, interventions were suggested that have been previously 
shown to positively influence the proposed constructs involved in science and chemistry identity formation. 


\section{Acknowledgements}

First and foremost, I need to say thank you to the instructors who welcomed me into their courses, either physically or virtually, to collect data in their classrooms. Time is precious in the classroom, and the two minutes it takes for student recruitment is priceless for researchers like me. Without your willingness to participate, this research would not be possible.

To my incredible advisor, Dr. Jack Barbera, I will always be grateful for the support you provided while I transitioned from analytical chemistry to chemistry education. You introduced me to a field that I absolutely fell in love with. I hope to stick around for a while and hope that our work together continues.

To my committee, thank you for your contributions to this work and to my overall experience in the Ph.D. program. Specifically, Dr. Erin Shortlidge for modeling the importance of qualitative work, Dr. Dean Atkinson for providing mentorship while allowing me to teach alongside you, and Dr. Jason Newsom for always making yourself available to my panic-ridden questions surrounding structural equation modeling. You all provided invaluable insight that helped me to grow as both a researcher and educator.

Chemistry education research doesn't always provide opportunities to be in the classroom. Thank you to my research assistantship advisor, Dr. Gwen Shusterman, for providing me with funding through the HHMI to work on a project that gave me many opportunities to implement activities within large classrooms, reminding me how much I love interacting with students. On top of providing me with research support, you were oftentimes a rock for me, helping to validate feelings that I often had trouble working 
through. I will always be thankful for the professional and emotional support you provided me.

To my DBER family, thank you for making research fun. The Barbera CER group consisting of Dr. Regis Komperda, Dr. Cory Hensen, Emryse Geye, Nicole Naibert, Andrew Isom, Amanda Schmidt, Gosia Cox and the BER group consisting of Brie Tripp, Emma Goodwin, and Liz Rain, it's been a pleasure going through this process with all of you and the feedback you've provided along the way has made my work so much better. Amanda, thank you for your contribution to phase one of this project. It was a pleasure working (and chatting about life) with you. Brie and Andrew, thank you for providing the validation and strength I needed when I was struggling with my mental health. Cory, thank you for making sure the CER and BER groups gather periodically so that we never felt like we were alone. Thank you Regis, for being the role model I desperately needed and the best mentor anyone could ask for.

To my grad-school sisters, Dr. Capri Price, Lyndsay Kissel, and Dr. Natasja Swartz, thank for your being my cheerleaders. Capri and Lyndsay, your daily chats made sure I was connected to the outside world, which can be easily forgotten in this process. Tasja, thank you for keeping in touch and periodically checking in to provide motivational support. To my village of friends and family, thank you for always providing a shoulder to lean (or cry) on. Christie and Brian, thank you for continuing to be our local family. Thank you to my mom, dad, Renée, Chris, Ben, Jessica, Annie, Dave, Ant (yes, ant) Karen, and Grandma for always providing safe havens when I visited and not asking (too many) anxiety inducing questions about grad school. To my 
four nieces, only two of who may actually remember my time in grad school, thank you for making me feel like a rockstar aunty and scientist. To my post-doc advisor, Dr. Joi Walker, thank you for allowing me the flexibility to focus on my dissertation while starting on an exciting new journey.

To my partner in life, Dr. Eric Young, there are so many things I could thank you for, but I'll try to keep it short before this acknowledgements section gets out of hand. We both started this journey together, but separate. Although two people in graduate school under one roof could be hell at times, I am so glad that we were able to go through this process together. I've learned so many things from you about discipline, hard work, and dedication. The best thing that this Ph.D. experience has given me, is you (and of course, our non-human children, Me-Mow, Mia, and Lindy).

Last, but absolutely not least, thank you to my therapist, for keeping me grounded when I was floundering. If you're reading this, you're probably aware that graduate school is hard. If you're struggling, talk about those struggles. If you find that your feelings aren't being validated, keep talking until you find someone who validates them. Hang on to that person. Graduate school can be an isolating endeavor, but it doesn't have to be. The most important lesson I've learned, from all of my experiences within this $\mathrm{Ph} . \mathrm{D}$. work, is that no matter how alone you feel, you are not alone. 


\section{Table of Contents}

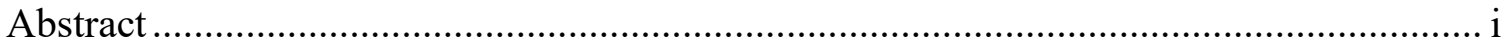

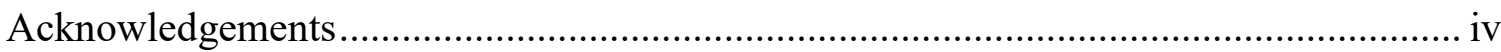

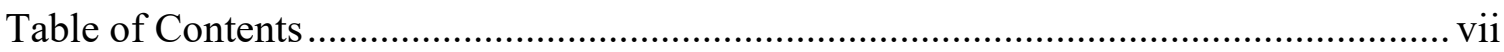

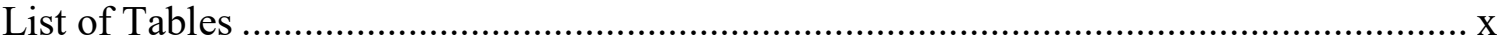

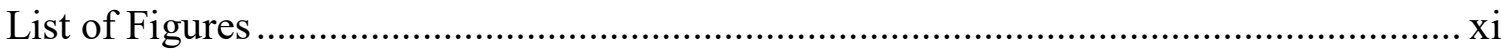

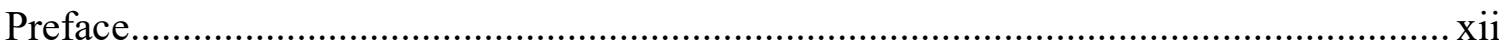

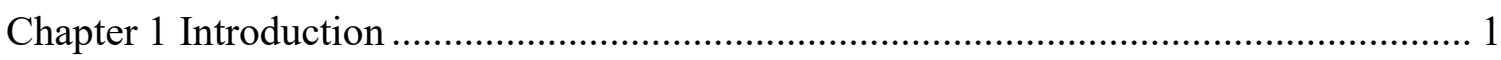

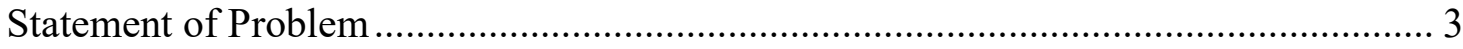

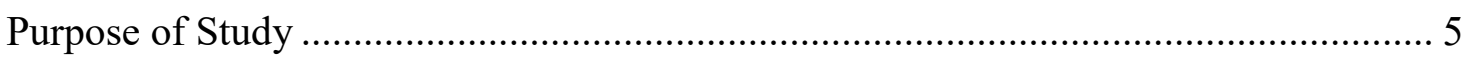

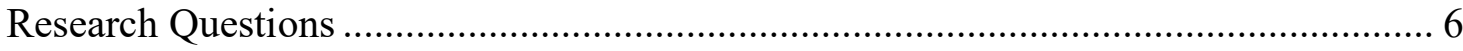

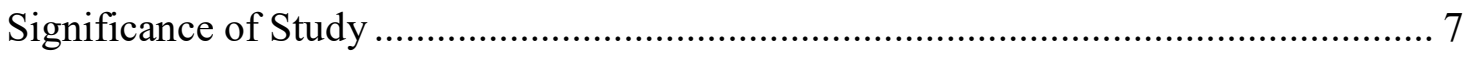

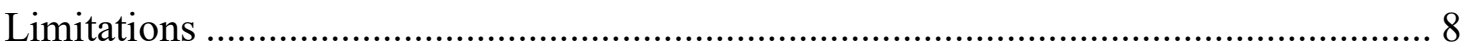

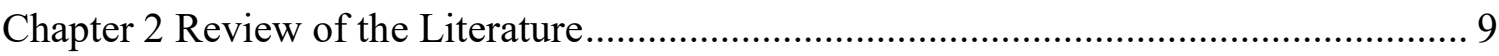

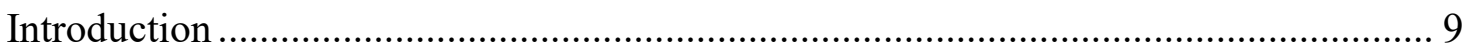

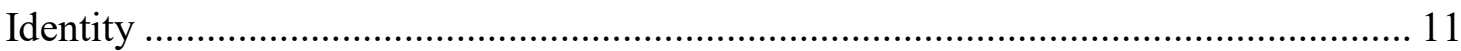

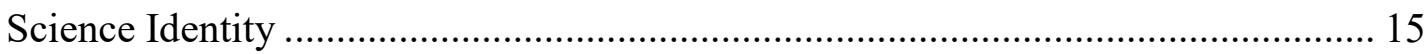

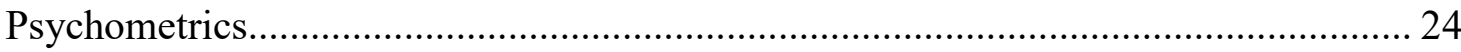

Validity ...

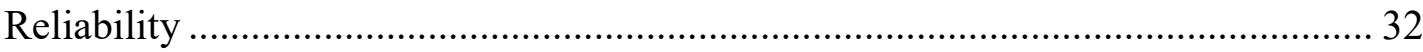

Existing Science and Discipline-Specific Identity Scales and Measures ................... 37

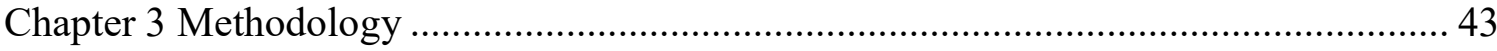

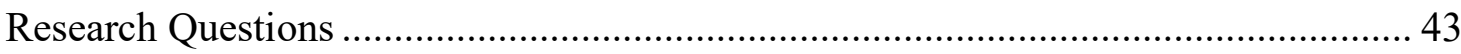

Phase One: Investigating the Overlap of the Sub-Constructs of the Physics Identity Framework With Established Affective Constructs......................................................... 44

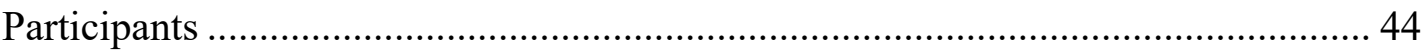

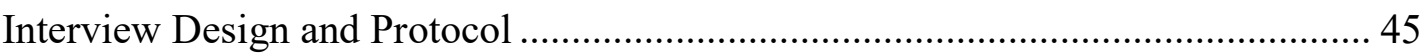

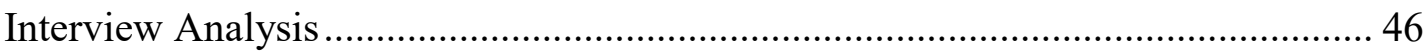

Phase Two: Testing the Psychometric Properties of and Relations Between Scales of Mastery Experience, Verbal Persuasion, and Situational Interest in Undergraduate

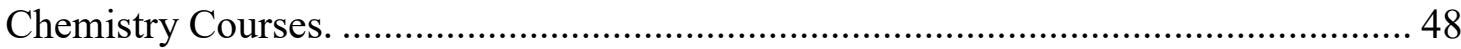

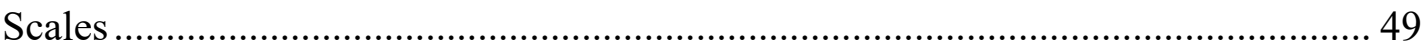

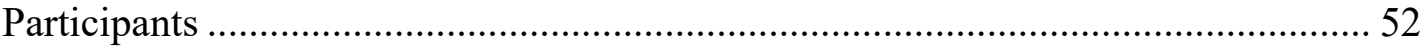

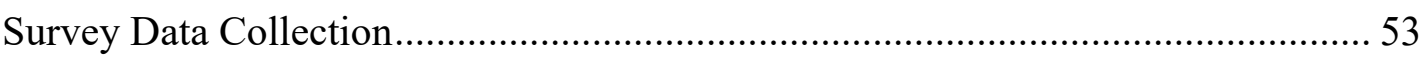

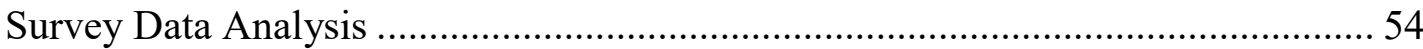

Chapter 4 Alignment of Theoretically Grounded Constructs for the Measurement of

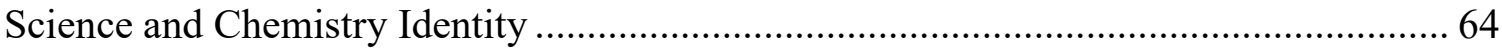

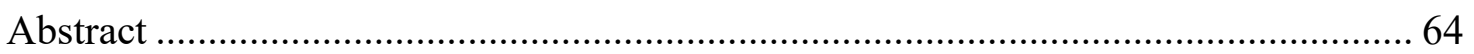




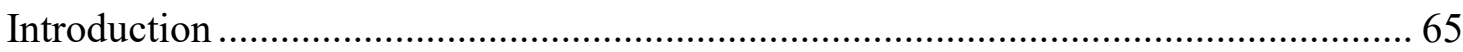

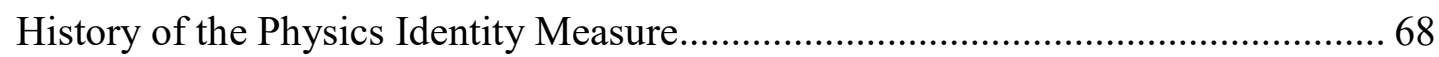

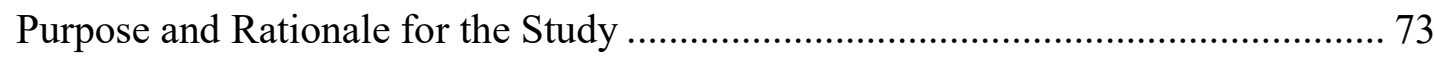

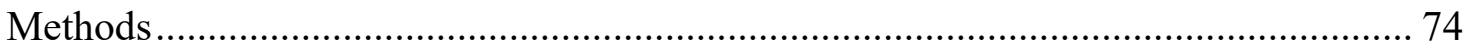

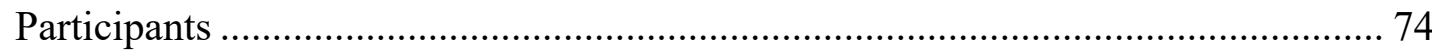

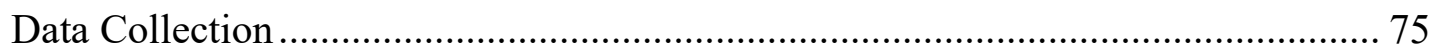

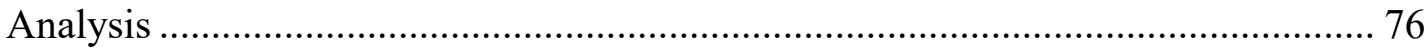

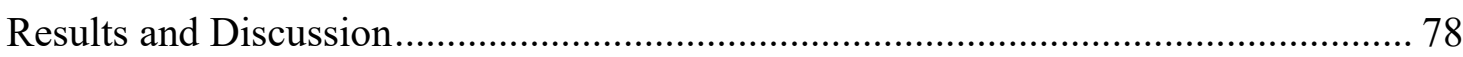

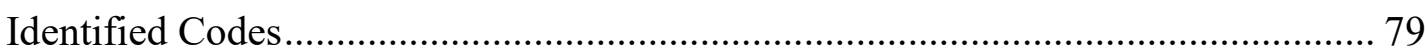

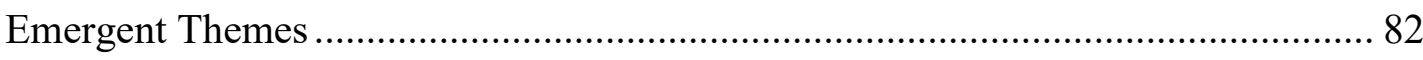

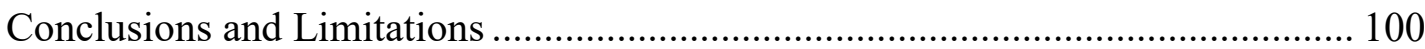

Chapter 5 Development and Evaluation of Novel Science and Chemistry Identity

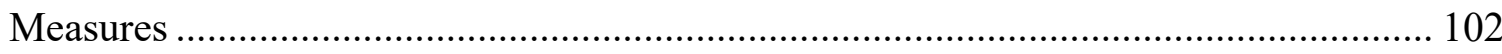

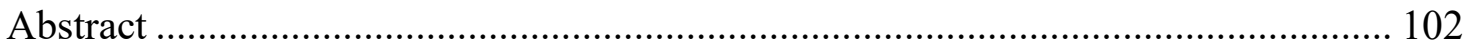

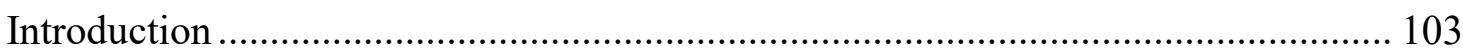

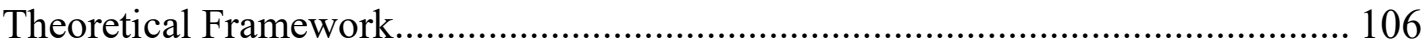

Purpose and Rationale for the Study ............................................................... 110

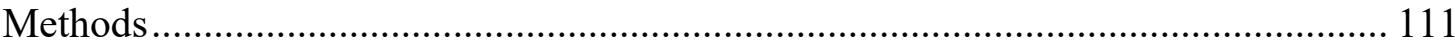

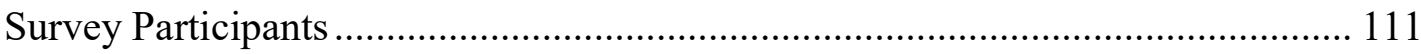

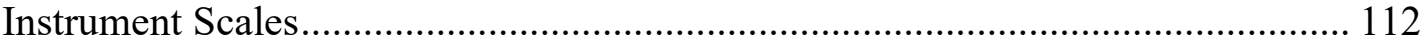

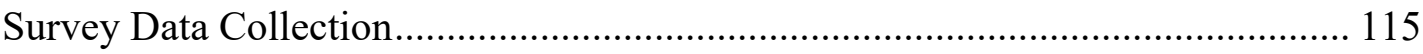

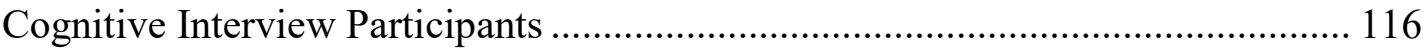

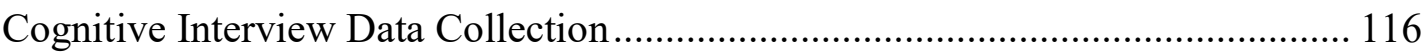

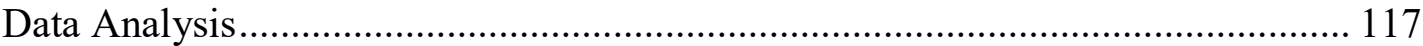

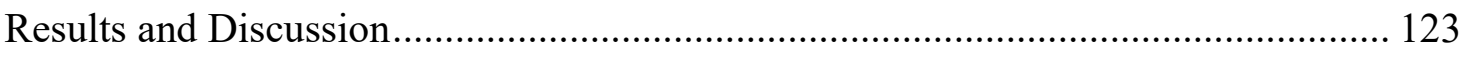

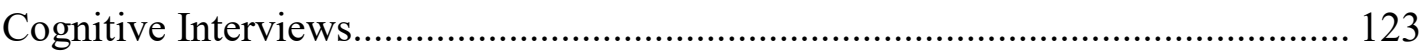

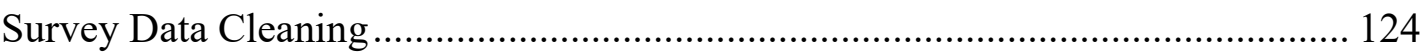

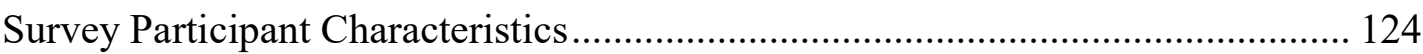

Descriptive Statistics and Response Patterns ...................................................... 124

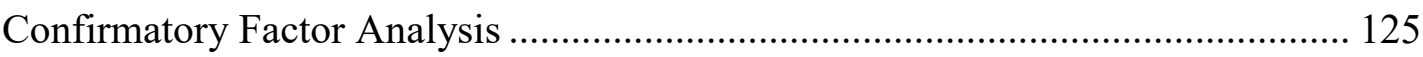

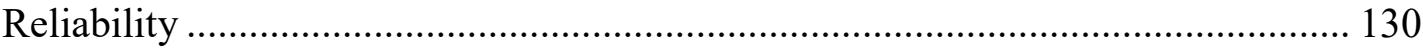

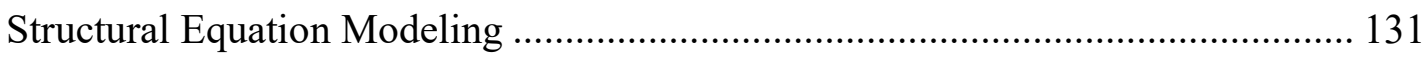

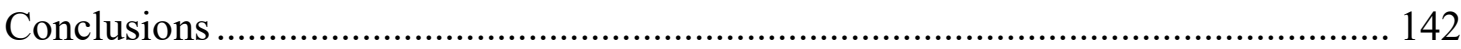

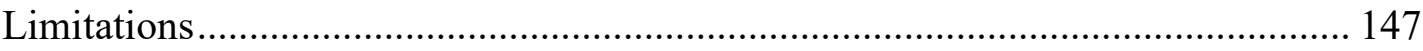

Chapter 6 Conclusions, Implications, Limitations and Future Research........................ 149

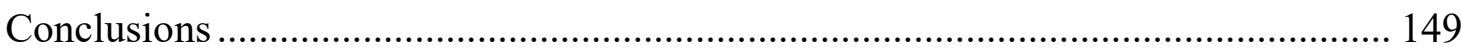

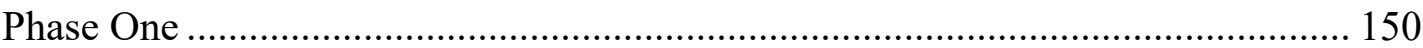

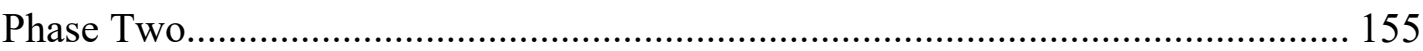

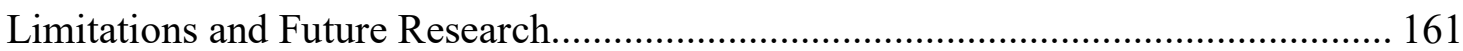


Implications for Researchers ..................................................................... 165

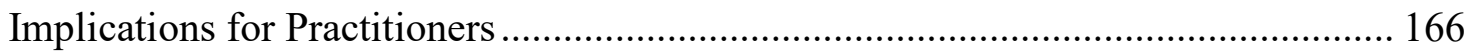

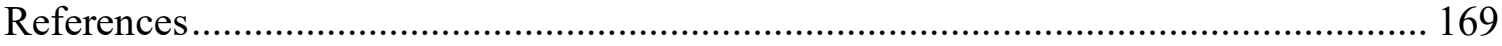

Appendix A: List of Questions and Demographics Used in Phase One....................... 180

Appendix B: Phase Two Additional Information ........................................................ 182 


\section{List of Tables}

Table 4.1. Division of interviews into sections by grouped items. The word [science] was replaced with chemistry for the chemistry-worded version of each question.. 78

Table 4.2. Pseudonyms and demographics for interview participants.......................... 79

Table 4.3. Codes, their definitions, and examples of codes within the text..................... 80

Table 4.4. The number of interviews and sections that contained each individual code.. 82

Table 4.5. Themes, codes contained within each theme, and a description of each theme that are present throughout the interviews. ................................................. 83

Table 4.6. Student quotes pertaining to feeling- and value-related interest.................... 85

Table 4.7. Student quotes pertaining to varying stages of interest. ............................... 87

Table 4.8. Student quotes pertaining to educational experiences in relation to science or chemistry identity.............................................................................. 92

Table 4.9. Student quotes pertaining to their interactions with others to navigate their science or chemistry identity..................................................................... 94

Table 4.10. Student quotes pertaining to the types of people who participate in science or

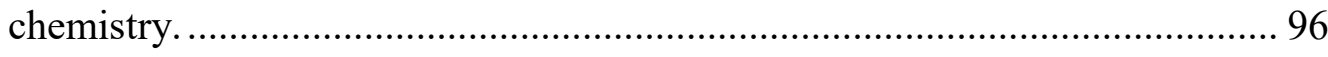

Table 5.1. Model fit for correlated two-factor feeling- and value-related interest models with error correlations.

Table 5.2. Model fit for correlated two-factor mastery experience and verbal persuasion models with error correlation.

Table 5.3. Model fit for correlated three-factor measurement models consisting of feeling-related initial or maintained interest, verbal persuasion, and mastery experiences.

Table 5.4. Omega values for time 1 and time 2 one-factor models.

Table 5.5. Fit indices for metric invariance testing between correlated one-factor models at time 1 and time 2 . 133

Table 5.6. Model fit for baseline and alternative structural equation models................ 134

Table 5.7. Fit indices for full SEM model metric invariance testing between courses.. 136 


\section{List of Figures}

Figure 2.1 A snapshot of the contemporary view of validity. ................................... 26

Figure 3.1. Proposed Phase Two SEM models ............................................................. 63

Figure 4.1. The theoretical frameworks for A) science identity, B) physics identity, and

C) physics identity after modification.................................................... 70

Figure 4.2. The alignment and modification of identity theories. ............................... 99

Figure 5.1. Affective constructs proposed to have an important role in identity formation as proposed by A) Carlone and Johnson (2007), B) Hazari and colleagues (2010), and C) Hosbein and Barbera (2020)............................................... 105

Figure 5.2. A) Baseline and B) alternative models with proposed relations between recognition, performance/competence, interest, and identity based on the physics identity framework................................................................. 108

Figure 5.3. Proposed baseline and alternative models for SEM................................ 121

Figure 5.4. Baseline and alternative SEMs after the removal of initial and maintained value-related interest based on CFA results............................................... 134

Figure 5.5. Metric invariant alternative SEMs for science worded identity constructs for general and organic chemistry. 138

Figure 5.6. Metric invariant alternative SEMs for chemistry worded identity constructs

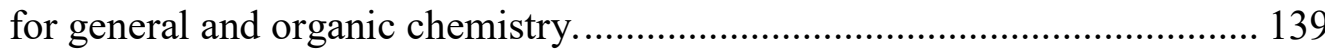


Identity, as an analytic lens, challenges and excites science education researchers. The challenge lies in the difficulty of theorizing it in empirically accessible and conceptually rigorous ways. The excitement lies in its explanatory potential for equity in science education.

-Heidi Carlone, 2017 


\section{Chapter 1}

\section{Introduction}

The phrase "I hated chemistry in school," is a very common response I hear when someone asks what I study. This is part of the reason why the research contained in this dissertation sparked my interest. It initiated the questions of, "Why do many people find it hard to identify with chemistry and other scientific fields in the way that they would with other popular fields such as art and business? Does this play a large part in why students either choose not to pursue or persist within STEM fields?" What type of person are you? An art person? A business person? A science person? The groups with which you identify define a portion of your overall identity as a human situated within society (Abrams \& Hogg, 1990; Gee, 2000; Lave \& Wenger, 2002; Stets \& Burke, 2000). This, in turn, may influence the way you navigate through life e.g., the career path you take.

What makes someone an art person, a business person, or a science person? The answer to these questions lies within the study of identity. Identity research has been prominent over the past couple of decades, specifically within education, (Barton et al., 2013; Brickhouse, Lowery, \& Schultz, 2000; Carlone \& Johnson, 2007; Chemers, Zurbriggen, Syed, Goza, \& Bearman, 2011; Gee, 2000; Graven \& Heyd-Metzuyanim, 2019; Hazari, Sonnert, Sadler, \& Shanahan, 2010; Kim, Sinatra, \& Seyranian, 2018; Nasir \& Saxe, 2003; Stets, Brenner, Burke, \& Serpe, 2017; Verhoeven, Poorthuis, \& Volman, 2018) as education plays a large part in what type of person one will become, in terms of their place within society (Chickering \& Reisser, 1993). Additionally, there are several recent dissertations that focus on identity (Hall, 2018; Johansson, 2018; Laskasky, 
2018; Palmer, 2018; Pelaez, 2017; Quan, 2017; Quon, 2018; Vincent-Ruz, 2019), indicating that research on identity is still very active.

There are many different ways that identity has been conceptualized (Gee, 2000; Hogg \& Terry, 2000; Lave \& Wenger, 2002) but a commonality that lies within each of these specific theories is that a portion of one's overall identity is socially constructed. These socially constructed identities have been referred to with terms such as social identity (Hogg \& Terry, 2000), role identity (Stets, 1995), and collective identity (Eccles, 2009), but the definition of these terms are all based on the concept of social formation of a particular identity rooted within membership to a specific group.

In order study the change in identity, i.e., to measure it, theory must describe identity in a clear and concise way, known as operationalization. An individual's overall identity contains many different facets and would be too complicated to operationalize in simplistic terms but, if we narrow it down and have enough information about an individual's identity situated within a specific context, such as a science, we can begin to operationalize these types of identities. Different microclimates are theorized to affect science identity, for example, at home, in the classroom, and outside of school (Aschbacher, Li, \& Roth, 2010). Support from multiple microclimates can help to bolster science identity. If an identity, such as science identity, is operationalized in a way that can be assessed, we can study what types of microclimates foster science identity and if differences in science identity formation exist between different populations.

This research within this study was focused on science and chemistry identity. Science and chemistry identity being defined as whether or not someone feels like a 
"science" or "chemistry" person (Gee, 2000). The focus of the studies contained within this dissertation was to contribute to the understanding of science and chemistry identity formation. More specifically, to more firmly situate an existing discipline-specific identity theory, the physics identity theory (Hazari et al., 2010), into established theoretical frameworks. Until high-quality instruments exist to assess aspects of science and chemistry identities, we cannot answer questions about how these identities vary across subpopulations of students or what type of learning environments can aid in fostering these identities.

\section{Statement of Problem}

While students often select their career trajectory before entering college (Bandura, Barbaranelli, Caprara, \& Pastorelli, 2001; Eccles, 2007), there is still a drastic discrepancy between the number of students entering college with a declared STEM major and graduating with a STEM degree (National Research Council, 2012; President's Council of Advisors on Science and Technology, 2012). This is especially an issue for underrepresented groups such as women, black, and Hispanic students (BonousHammarth, 2000; Koenig, 2009; Penner, 2015; Seymour, Hewitt, \& Friend, 1997). One of the proposed mechanisms to increase persistence within the STEM fields is to foster science identity (Estrada, Woodcock, Hernandez, \& Schultz, 2011; Flowers \& Banda, 2016; Graham, Frederick, Byars-Winston, Hunter, \& Handelsman, 2013; ShedloskyShoemaker \& Fautch, 2015).

Instruments that take the form of surveys are used to assess psychological constructs such as science and chemistry identities. There are several instruments that aim to measure a type of science or STEM discipline-specific identity. These include 
scientist identity (Chemers et al., 2011; Estrada et al., 2011), science student identity (Stets et al., 2017), or discipline-specific identities such as physics, math, and engineering (Cass, Hazari, Cribbs, Sadler, \& Sonnert, 2011; Godwin, Potvin, \& Hazari, 2013; Hazari et al., 2010). One identity measure was designed to address one particular intervention and is therefore useful for assessing the impact of this very specific intervention rather than assessment of the overall learning environment (Childers \& Jones, 2017). Brickhouse and colleagues (2000) have stressed the importance of studying appropriate communities of practice in which science identities can form. For example, the term scientist could pose a narrow view of what it means to engage in science as it implies that an individual is actively engaging in science outside of the classroom. Studying a research scientist community of practice (i.e., scientist rather than science student or science person) in relation to the formation of students' science identity could be problematic as it could be irrelevant to students, depending on their experiences with science outside of school. Although science and discipline-specific identity measures currently exist, many lack the appropriate context or are not designed to specifically measure chemistry identity.

A crucial aspect of assessment instrument development is to have evidence that the instrument is measuring what it is intended to measure. Therefore, the first step in instrument development is to choose a theoretical framework on which to base the measure (Kline, 2016). In the past, science identity was broadly defined and therefore, not operationalized in a way that aligned with creating a measure. Carlone and Johnson (2007) attempted to present a more clearly defined theory of science identity as it 
pertained to women of color but not for the intention of measurement. Hazari and colleagues, (2010) modified and operationalized this theory to a physics identity framework for the purpose of measuring physics identity. While the physics identity framework was based on an established theory of science identity, the alignment of the items used to measure identity was unclear. This led to a measure that was designed without clear connections to a theoretical framework.

\section{Purpose of Study}

There is evidence to show that different learning environments can aid in fostering aspects of science identity (Kim et al., 2018). Much of this evidence has been provided based on in depth qualitative research and through a critical theory lens, meaning that each study is very individualized. The goal within this research, was to provide initial steps toward a more theoretically grounded and generalizable quantitative measure that could be used to evaluate multiple aspects of science and chemistry identities within different learning environments at the college level.

While the physics identity framework (Hazari et al., 2010) was built upon a science identity theoretical framework (Carlone \& Johnson, 2007), the development of the items to measure identity, i.e., the alignment between items and theory, was unclear. The physics identity framework aimed to measure three aspects of identity: performance/competence, interest, and recognition. The lack of alignment between items designed to measure these constructs and corresponding theoretical frameworks provided a starting point for this investigation.

The overlap of performance/competence, interest, and recognition with corresponding theoretical frameworks was investigated through four objectives. The first 
and second objectives were explored through a qualitative study that consisted of, first, determining themes that arose when general and organic chemistry students were asked about the constructs within the physics identity framework and, second, exploring overlap between the themes and corresponding established theoretical frameworks. The third and fourth objectives of the study were investigated quantitatively. Measures of the established theoretical frameworks from the second objectives were chosen and modified to fit the context of this study. The psychometric properties of these measures were then investigated before moving to the fourth objective, which was to study the relations between the measures and the extent to which a student felt like a "science" or "chemistry" person. Structural equation modeling (SEM) was used to create models through which to investigate these relations.

\section{Research Questions}

The primary goal of this study was to more clearly align the physics identity framework with constructs that are prominent in psychological literature. The secondary goal of the project was to operationalize the instrument to science and chemistry identities. The objectives of this project were carried out by the following research questions:

Q1. What themes arise when students are asked questions reflecting performance/competence, recognition, and interest, pertaining to science or chemistry, as described by the physics identity framework?

Q2. To what extent do reported themes align with proposed and other affective constructs? 
Q3. To what degree will an instrument containing items designed to measure mastery experiences, verbal persuasion, and situational interest show psychometric functionality in undergraduate chemistry courses with

a. science-specific wording?

b. chemistry-specific wording?

Q4. What are the relations between mastery experiences, verbal persuasion, situational interest and a

a. science identity indicator?

b. chemistry identity indicator?

\section{Significance of Study}

This study contributes to the chemistry education community by expanding on the understanding of science identity formation and investigating chemistry identity formation. According to the narrative theory of identity, which defines identity in terms of stories told by those during their identity formation, "It is now not unreasonable to conjecture that identities are crucial to learning. With their tendency to act as selffulfilling prophecies, identities are likely to play a critical role in determining whether the process of learning will end with what counts as success or with what is regarded as failure (Sfard \& Prusak, 2005, p. 19)." While there are identity measures in the literature, the most prominent measure (Godwin, Potvin, \& Hazari, 2013; Hazari et al., 2010), based on the physics identity framework, lacks a clear connection between the items used to measure identity and established theoretical frameworks, which is a fundamental aspect of instrument development. Identity, as defined within this study depends on group membership. Based on this definition, it is important to investigate whether "science" and 
"chemistry" identities differ. A chemistry-specific identity measure, to use for comparison to science identity, does not currently exist. Studying the relations of constructs within science and chemistry identity can provide insight into the types of learning environments that may help to foster identity formation.

\section{Limitations}

There are multiple limitations within the present study. This research was performed with convenience sampling from general and organic chemistry classrooms at five U.S. universities and therefore, has limited generalizability to other chemistry classrooms across the U.S. Additionally, interview participants consisted of a small sample from a various chemistry courses at a single institution. Although data saturation was reached, the views of these students may not represent the entire population under study. Lastly, this research is operationalized within a specific theoretical framework. It is possible that valuable constructs related to science and chemistry identity will be left out, such as a sense of belonging (Cheryan, Master, \& Meltzoff, 2015), stereotype threat (Shapiro \& Williams, 2012), and the specific environments that inform identity development (Kim et al., 2018). 


\section{Chapter 2}

\section{Review of the Literature}

\section{Introduction}

The fact that a majority of STEM students do not complete their STEM degree has been documented for some time. In 2012 , less than $40 \%$ of students who began college with the intent of majoring in STEM actually obtained a STEM degree (President's Council of Advisors on Science and Technology, 2012). This issue has been so prominent that the President's Council of Advisors on Science and Technology (PCAST) compiled a report to the President calling for an additional one million college graduates in STEM by 2022 to keep up the need predicted by economic forecasts (2012).

Those leaving are already disproportionately under-represented in the STEM population, including groups such as black and Hispanic students and women (BonousHammarth, 2000; Koenig, 2009; Penner, 2015; Seymour et al., 1997). The PCAST report (2012) made a stunning observation. While minority groups made up approximately $70 \%$ of all college students, they made up less than $45 \%$ of those who obtained STEM degrees. This statistic adds a layer of complexity to understanding why students leave STEM and how to help them persist.

The PCAST report proposed ways to increase student retention in STEM. One of the recommendations within the report included catalyzing widespread adoption of evidence-based teaching practices. As the name implies, evidence based-teaching practices are teaching practices that have evidence to support their effectiveness. Active learning is commonly cited as an evidence-based teaching practice. Active learning is a broad term used to describe activities that involve students engaging directly with 
learning material, as opposed to being passively 'delivered' information. While there are still many questions about what types of active learning are the most effective, there is strong evidence that active learning modalities are more effective than traditional (passive) lectures in general (Freeman et al., 2014)). The persistence framework (Graham et al., 2013) proposes using active learning along with early research and learning communities to encourage learning and identification with being a scientist, which in turn will provide confidence and motivation for students to persist in STEM.

While evidence supports that active learning is more effective than traditional learning, the specific characteristics of active learning that are responsible for this difference in effectiveness are not as clear. One of the aims of Discipline-Based Education Research (DBER) is to provide high quality assessments to measure the impacts of active learning (National Research Council, 2012, p. 3). There are two domains that are commonly assessed in order to provide evidence of effective teaching practices: cognitive and affective (Bloom, 1956; Kratwohl, Bloom, \& Masia, 1964). The cognitive domain includes assessment of the acquisition of knowledge. This evidence can be presented in the form of assessments that measure certain learning gains. The affective domain is not concerned with the acquisition of knowledge directly but focuses on psychological constructs such as attitudes, interests, and beliefs that are related to the acquisition of knowledge. Changes in the affective domain have been shown to relate to multiple variables such as student performance as well as persistence in college (Robbins et al., 2004). 
Affect plays such a crucial role in learning and persistence that an increase in research on affective domains within STEM education has been called for (Fortus, 2014; Graham et al., 2013; Jones, Corin, Andre, Childers, \& Stevens, 2017; President's Council of Advisors on Science and Technology, 2012; Trujillo \& Tanner, 2014). It is not realistic to ask every student why they persist or do not persist within STEM. In order to understand the impacts that learning environments have on changes in affect in a somewhat generalizable way, high quality instruments must be used. Some aspects of the affective domain such as motivation, interest, and self-efficacy have multiple instruments associated with them (Ferrell \& Barbera, 2015; Glynn, Brickman, Armstrong, \& Taasoobshirazi, 2011; Liu, Ferrell, Barbera, \& Lewis, 2017). These affective constructs have varying but strong theoretical backgrounds, rooted in psychology literature, which provides a basis for high quality instrumentation. Motivation, for example, can be examined through the lens of several theories including self-determination (Ryan \& Deci, 2000), expectancy value (Eccles \& Wigfield, 2002), or social cognitive theory (Bandura \& National Inst of Mental Health, 1986) perspectives to name a few. While instruments pertaining to some affects exist, there is still a lack of high quality instruments pertaining to affective constructs that can influence identity, which has been theorized to be critical for persistence in STEM (Estrada et al., 2016; Flowers \& Banda, 2016; Graham et al., 2013).

\section{Identity}

If I were to ask, "What makes you, you?" it may take a while to come up with an answer. This is because an individual's identity is complicated. An individual can have multiple identities based on their race (Sellers, Smith, Shelton, Rowley, \& Chavous, 
1998), gender (Davidoff \& Hall, 1987; Lorber, 1994), social status (Davidoff \& Hall, 1987), etcetera. Identity can be socially constructed (Abrams \& Hogg, 1990) as well as individually constructed (Burke \& Stets, 2009). The complexity of identity has made it a focus in psychological research and there are several lenses through which to view identity. Theories that are most relevant to this dissertation are highlighted within this section.

Identity was initially proposed as a lens for education research by Gee (2000). Within this proposal was an approach to conceptualizing identity while recognizing that alternate approaches exist within the literature.

"When any human being acts and interacts in a given context, others recognize that person as acting and interacting as a certain "kind of person" or even as several different "kinds" at once... A person might be recognized as being a certain kind of radical feminist, homeless person, overly macho male, "yuppie," street gang member, community activist, academic, kindergarten teacher, "at risk" student, and so on and so forth, through countless possibilities. The "kind of person" one is recognized as "being," at a given time and place, can change from moment to moment in the interaction, can change from context to context, and, of course, can be ambiguous or unstable.

Being recognized as a certain "kind of person," in a given context, is what I mean here by "identity." In this sense of the term, all people have multiple identities connected not to their "internal states" but to their performances in society. This is not to deny that each of us has what we might call a "core identity" that holds more uniformly, for ourselves and others, across contexts (p. 99)."

Gee then went on to describe four perspectives through which identity could be viewed. These four perspectives are not separate from one another but rather give perspectives through which to formulate questions about identity formation.

The four perspectives within Gee's (2000) description of identity include natureidentity, institution-identity, discourse-identity, and affinity identity. All four perspectives 
include a process, a power, and a source of power. Nature-identities (N-identities) are considered a state (process) developed from forces (power) in nature (source of power). For example, an individual's identity as a twin would be considered an $\mathrm{N}$-identity. This identity type cannot be controlled by the person or society, as it was pre-determined by nature. Institution-identities (I-identities) consist of a position authorized by authorities within institutions, such as an individual's identity as a professor. This identity was authorized by (process) authorities (power) to the individual through a university (source of power). Discourse-identities (D-identities) consist of individual traits recognized in the discourse or dialogue of/with "rational" individuals. For example, an individual can identify as a funny person and can gain this identity by recognition (process) of their actions (power) by others who surround them (source of power). Affinity-identities (Aidentities) are comprised of experiences shared in the practice of "affinity groups", where an affinity group is defined as a group made up of people who may be separated physically but share a common interest and participate in practices that define the group. An example of an A-identity would be a "Trekkie" identity. A Trekkie is someone who is a super-fan of the television series Star Trek. These specific fans participate in (process) practices such as attending Star Trek conventions (power), participating in online forums about Star Trek, meeting actors from the show, and dressing up as characters from Star Trek and therefore recognize each other (source of power) as Trekkies. The participants in this group may hold little else in common except for their love of Star Trek and this is what holds together their A-identity as a Trekkie. 
Gee's four perspectives of identity are not necessarily neatly divided into separate identities for an individual. For example, someone who has a professor identity may originally have this identity because a university deemed them a professor (I-identity). However, over time, their actions may be recognized by others (such as students and other professors) as those that reflect a type of person who is a professor (D-identity). Therefore, this I-identity could shift to a D-identity as the individual is recognized by others surrounding them. While these four perspectives differ in the power that recognizes the identities, recognition is a common thread throughout the perspectives. Identities must be recognized in order for them to exist at all. Recognition is tied to "Discourse" with a capital "D", which is a concept that weaves through all types of identity perspectives. Discourse is described as the "ways of being 'certain kinds of people (p. 110).' " This concept is similar to communities of practice (Wenger, 2000) and includes the way someone presents themselves in the context of their identity. For example, being a professor is a different Discourse than being a student.

Gee (2000) then proposes the implications of the four perspectives of identity on identities such as race, gender, class, and ability. Identities are interactional achievements where individuals can gain and lose recognition as certain "types of people". This is the realm of D-identities, where the identities are negotiated across differing social groups. Identities also involve positions in society deemed by institutions. For example, universities assign what an acceptable student should be and therefore play a part in shaping a "student identity". This is where I-identities come in. N-identities address the fact that individuals can be recognized based on "natural categories" such as their 
biology, which can include things such as race, or ability. Lastly, individuals can build identities based on group activities to form A-identities. These perspectives of identity work both separately and together to form the different identities that an individual can hold.

The complexity of identity makes it a construct that is hard to operationalize, leading many researchers to focus on multiple identity frameworks within each research study. The aforementioned conceptualization of identity involves social construction within a contextualized setting. When conceptualizing identity in this way, we can start thinking of contexts that exist within an individual's education in which they may form a specific identity.

\section{Science Identity}

Researchers use many different terms for what will be referred to from here on as 'role identity'. This type of identity is contextual to a specific domain such as a Discourse (Gee, 2000) or community of practice (Wenger, 2000). Examples of role identities include gender identity (Stets, 1995), academic identity (Osborne, 1997), science identity (Brickhouse, 2000; Carlone \& Johnson, 2007), and discipline-specific identities such as physics (Hazari et al., 2010), engineering (Godwin, Potvin, \& Hazari, 2013), and math (Cribbs, Hazari, Sonnert, \& Sadler, 2015) identities.

\section{Science Identity Formation}

The study of the process by which identity is formed is prevalent in education literature. Many studies that focus on science or discipline-specific identity formation are qualitative and longitudinal. These studies have historically focused on middle school (Barton et al., 2013; Brickhouse, 2000) and high school students (Aschbacher et al., 
2010; Basu, Barton, Clairmont, \& Locke, 2009; Brickhouse \& Potter, 2001; Sfard \& Prusak, 2005), however, the number of studies focused on post-secondary students and beyond are on the rise (Carlone \& Johnson, 2007; Godwin \& Lee, 2017; Hyater-Adams, Fracchiolla, Finkelstein, \& Hinko, 2018; Jackson \& Seiler, 2013; Li \& Loverude, 2013; Robinson, Perez, Nuttall, Roseth, \& Linnenbrink-Garcia, 2018). A majority of qualitative studies within the literature surrounding identity formation follow students who belong to an underrepresented minority population including women and ethnic minorities, as these are populations most likely to leave STEM fields. Poststructural feminist epistemologies are commonly used in science identity formation and therefore these studies often raise questions about scientific knowledge, objectivity and the oversimplifications of gender (Brotman \& Moore, 2008). These types of studies were, in part, a response to critiques that state that "white females are the norm for gender issues" when intersectionality, such as race, class, gender, religion etc., was not considered (Brotman \& Moore, 2008; Monroe, 2000). Understanding how science identity is formed and sustained, with intersectionality considered, is crucial to providing learning environments that could aid in improving the retention of underrepresented populations within the STEM pipeline.

Brickhouse, Lowery, and Schultz (2000) published a seminal paper that followed four African-American girls in $7^{\text {th }}$ grade for 18 months to explore the formation of science identity within the classroom in relation to the girls' social identities (e.g., race, gender, and class). The four girls identified positively with science in the classroom, but their social identities were found to conflict with the formation of science identity when the girls did not have a strong "good student" identity. For example, a "good student" 
identity would consist of doing what was asked by the teacher or staying silent throughout most of class. It was concluded that the girls may have benefitted from a "curriculum that permitted more diversity in the ways students might engage in and use science content (p. 456)" to avoid being forced to fulfill the stereotypical "good student" identity. This conclusion provides support for the need to understand how different groups of students identify with science to better create supportive learning environments.

Many in depth studies of science and discipline-specific identity formation have been conducted following the Brickhouse and colleagues (2000) study. At the postsecondary level, these studies focus on role identity formation both inside (Brickhouse \& Potter, 2001; Tonso, 2006) and outside (Hunter, Laursen, \& Seymour, 2007; Tate \& Linn, 2005) of the classroom. Despite the differences between identity perspectives used within each study, the part that identity formation plays in learning and persistence within STEM domains is a common link.

The qualitative studies mentioned here explored the formation of science identity but failed to give an expanded theory of what science identity consisted of beyond the strength of an individual identifying with science or seeing oneself as a person who engages in science. While qualitative studies on small samples provide rich and detailed personal experiences, which provide support for theories, it is not logical to perform these detailed studies with larger populations to make more generalizable conclusions about certain groups. These studies provided a strong foundation from which to build a science 
identity theory, taking intersectionality into consideration, but to move toward a theory that could provide a clearer analytic direction, these works needed to be expanded upon.

\section{Science Identity Expanded}

Responding to the amorphous nature of the way in which science identity had been described within studies, Carlone and Johnson (2007) proposed a science identity theory that involved an expanded definition. This theoretical framework was informed by both practical and theoretical sources as well as Gee's theory of identity (2000) and was developed to understand the experiences of 15 women of color through undergraduate, graduate, and the beginning of their science-related careers. Their initial hypothesized framework incorporated the constructs of performance, competence, and recognition into the 'facets' of an individual's science identity. Performance was defined as "social performance of relevant scientific practices-e.g., ways of talking and using tools (p. 1191)." Competence was defined as "knowledge and understanding of science content (may be less publicly visible than performance) (p. 1191).” Recognition was defined as "recognizing oneself and getting recognized by others as a 'science person' (p. 1191)." The three constructs were hypothesized to work together in a way that a person would perform tasks that illustrate their competence in a way that an individual would be recognized by others as a credible science person. This theory accounts for the socially constructed nature of science identity, as there are certain norms associated with science performance, competence, and recognition and therefore assumes that an individual's racial, ethnic, and gender identity may also influence science identity.

The women in the Carlone and Johnson (2007) study were recruited through a program supporting high-achieving students of color in science. Participants were 
ethnically and racially diverse including four Latinas, four black women, two American Indian women, and four Asian American women. Data was collected through ethnographic interviews during their sophomore (three participants) and junior or senior (twelve participants) years as well as follow up e-mail interviews six years later. Semantic structure analysis (Spradley, 1980) was used to search for patterns in the data that could be separated into categories with cultural meaning. Through taxonomic analysis (dividing the data into specific classifications), different types of recognition such as "recognition by others outside of science" and "recognition by meaningful scientific others" were found to be a crucial component to the formation of the women's science identities. Public performance of relevant scientific skills could not be analyzed through interviews and is why performance of the women was not analyzed. Recognition was chosen as the focus of further analysis rather than competence (measured by GPA), as it was the most distinctively different among women and could be used to distinguish experiences. Componential analysis (selecting the most relevant classifications) was then used to divide the types of recognition into three different types of science identity trajectories: research, altruistic, and disrupted science identities. Women in the research science identity trajectory had careers as research scientists. Altruistic science identity trajectories lead to careers in the fields of health. The women who had disrupted science identities did not enter doctoral programs and had varying careers in science, although they still had a strong orientation toward research. By comparing the varying science identities, Carlone and Johnson concluded that "developing a satisfactory science identity hinges not only upon having competence and interest in science, but also, critically, upon 
recognition by others as someone with talent and potential in science. Our focus on women of color, those who have not been recognized historically as 'science people,' brings into relief the importance and problematic nature of recognition by others in cultivating satisfying science identities (p. 1197)".

\section{The physics identity framework}

The Carlone and Johnson (2007) study highlights the intersectionality of science identity with other identities that may conflict with one another, such as racial identity. While the study developed a more clearly outlined science identity theory, it could not be generalized to larger populations. Carlone and Johnson's (2007) science identity theory was expanded and modified to create a physics identity theoretical framework (Hazari et al., 2010) with the aim of making more generalizations about underrepresented populations in STEM, such as women. This novel framework was strongly based on the theory of science identity introduced by Carlone and Johnson but also situated within the social cognitive career theory (SCCT) (Lent, Brown, \& Hackett, 1994) which was built upon social cognitive theory (SCT) (Bandura \& National Inst of Mental Health, 1986). The physics identity framework recognizes that a student's full identity is composed of personal identity, social identity, and identification with physics (role identity). The specific measure focuses on identification with physics. The sub-constructs of physics identity in this measure included recognition by others, competence, performance, and interest. The science identity sub-constructs (Carlone \& Johnson, 2007) were modified in three distinct ways to create the physics identity sub-constructs. First, within the science identity framework, recognition was conceptualized as a combination of self-recognition and recognition by others (Carlone \& Johnson, 2007). Within the physics identity 
framework, self-recognition was viewed as a core feature that could be influenced by whether an individual is recognized by others. Therefore, self-recognition was theorized as a separate and holistic identity variables consisting of a single item that asked, "Do you see yourself as a physics person?" (Potvin \& Hazari, 2013; Shanahan, 2009). Second, performance and competence were re-worded to reflect student confidence in ability (similar to self-efficacy) (Hazari et al., 2010) rather than actual ability (Carlone \& Johnson, 2007). The third modification was the addition of the construct of interest. Interest was not originally included in the science identity framework because the women who participated in the study were already on an established path to become scientists and therefore their interest did not need support (Carlone \& Johnson, 2007). However, Carlone and Johnson did describe interest as important to identity formation. The subconstruct of interest was added because their participants were not necessarily on an established path of science and interest has been shown to predict student career choices based on the SCCT (Hazari et al., 2010). Items based on these constructs have been developed and tested using exploratory factor analysis (EFA). After EFA analysis, a revised framework was proposed that included performance/competence as a single construct, recognition by others, the identity variables (i.e., self-recognition), and interest. This conceptualization of the physics identity framework has been subsequently used in further studies (Cass et al., 2011; Cheng et al., 2018; Cribbs et al., 2015; Godwin, Potvin, \& Hazari, 2013; Verdín, Godwin, Kirn, Benson, \& Potvin, 2018). While the discussion of identity is prevalent in education and psychological literature, it is not often the focus of study within chemistry education. As of October, 
2019, a search of the term "science identity", within the Journal of Chemical Education, brings up only five manuscripts, with all five only mentioning the term and not specifically using it in their study. A similar search for the term "chemistry identity" brought up zero manuscripts. Within the journal Chemistry Education Research and Practice, a search for the term "science identity" resulted in five manuscripts, with only one manuscript including science identity within their investigations. A search for "chemistry identity" resulted in four manuscripts, with zero investigating chemistry identity within the studies.

\section{Limitations of the Existing Science and Physics Identity Theories}

The physics identity framework include the constructs of recognition, performance/competence, and interest (Hazari et al., 2010). However, it is not clear in which theoretical frameworks these proposed constructs are grounded. Carlone and Johnson assigned definitions to the constructs of performance, competence, and recognition but did not ground each construct within a specific theoretical framework. The physics identity framework (Hazari et al., 2010) was then developed to operationalize the constructs to a discipline-specific framework, with slightly modified definitions, and added the construct of interest. In the physics identity framework, social cognitive career theory (SCCT) (Lent et al., 1994) was used to somewhat ground the entirety of physics identity, but the grounding of each specific construct in the context of SCCT is not discussed. SCCT is heavily based on Bandura's social cognitive theory (SCT) (Bandura \& National Inst of Mental Health, 1986). SCCT "emphasizes the means by which individuals exercise personal agency in the career development process, as well as extra-personal factors that enhance or constrain agency (Lent et al., 1994)". Within 
SCCT, performance and competence are not distinguished and are usually measured using grades. In the physics identity framework, performance was predicted to increase self-efficacy, which in turn could increase persistence, with does align with the description of performance within SCCT (Hazari et al., 2010). Within SCCT, selfefficacy and outcome expectations were proposed to influence interest, which in turn influenced goals (Lent et al., 1994). The items used to measure the construct of interest in the original physics identity measure (Hazari et al., 2010) reflect interest in specific tasks associated with a domain rather than containing interest items rooted in a specified theory of interest. In one study that uses the physics identity measure (Verdín et al., 2018), the author's quote a relevant interest description and state that learners who are interested in specific tasks "are likely to be able to self-regulate and persist to complete tasks even when they are challenged, whereas learners with little interest typically have difficulty engaging and continuing to work with tasks (Renninger, Nieswandt, \& Hidi, 2015, p. 2)”. While this statement generally spans multiple theoretical frameworks of interest, it does not specify a specific framework of interest from which this statement originated. Recognition is not specifically mentioned in SCCT but does align well with verbal persuasion source of self-efficacy within SCT (Bandura \& National Inst of Mental Health, 1986, pp. 405-406), although this is not mentioned by the authors. Because of the lack of clear theoretical connections to the sub-constructs of identity proposed within the science (Carlone \& Johnson, 2007) and physics (Hazari et al., 2010) identity frameworks, it is worth exploring the connection of other affective constructs within the literature to these constructs of identity. 


\section{Psychometrics}

We are assessed in various ways throughout our lives whether it be in the classroom as a student or teacher or in our general lives such as tests an individual must pass to obtain a driver's license. When a psychological attribute such as intelligence is measured, the instrument with which is it measured must be "calibrated". This is similar to the way that any analytical instrument in bench chemistry must be calibrated to the specific sample of interest. The way that psychological instruments (or measures) are "calibrated" involves the field of psychometrics.

Psychometrics is the science concerned with the attributes of psychological assessment. Systematic and random errors need to be accounted for every time an instrument is used with a new sample. When an analytical instrument is used to make chemical measures, accuracy would be used to assess systematic error and precision used to assess random error. Within the psychometric realm, accuracy and precision are analogous to validity and reliability. All instruments should show evidence of producing data that is both valid and reliable. A psychological instrument should also have attributes including standardization and a lack of bias (Rust \& Golombok, 2014). From here on, the term instrument will be used to describe a tool used to assess psychological attributes unless otherwise noted.

Psychological attributes can either be observable or not observable. For example, you can directly observe and measure certain behavioral traits such as facial expressions while it is not possible to directly measure IQ. Attributes that cannot be directly measured are called latent variables, or constructs as they will be referred to from here on (Furr \& Bacharach, 2008, p. 5). 


\section{Validity}

Validity is analogous to the accuracy of the data provided by an instrument in the sense that it accounts for systematic error contributing to the total measurement accuracy. There needs to be evidence to show that the data provided by an instrument is measuring what is was set out to measure. In other words, when an assessment is used to measure a latent construct, and the data shows evidence of validity, the interpretations of the assessment are more robust. It is important to note that validity evidence pertains to the data produced by an instrument, and not the instrument itself. Each time an instrument is used within a new environment, the data provided by the instrument needs to show evidence of validity. The conceptualization of validity has changed over time. In the past, validity was divided into distinct types (criterion, construct, content, etc,) (Cronbach \& Meehl, 1955). The contemporary view has been shaped into a single umbrella term called construct validity with multiple categories of validity evidence contained within (Geisinger et al., 2013), as shown in Figure 1. The categories contain evidence sources based on assessment content, response processes, internal structure, relations of the assessment with other variables, and consequences of the assessment. 


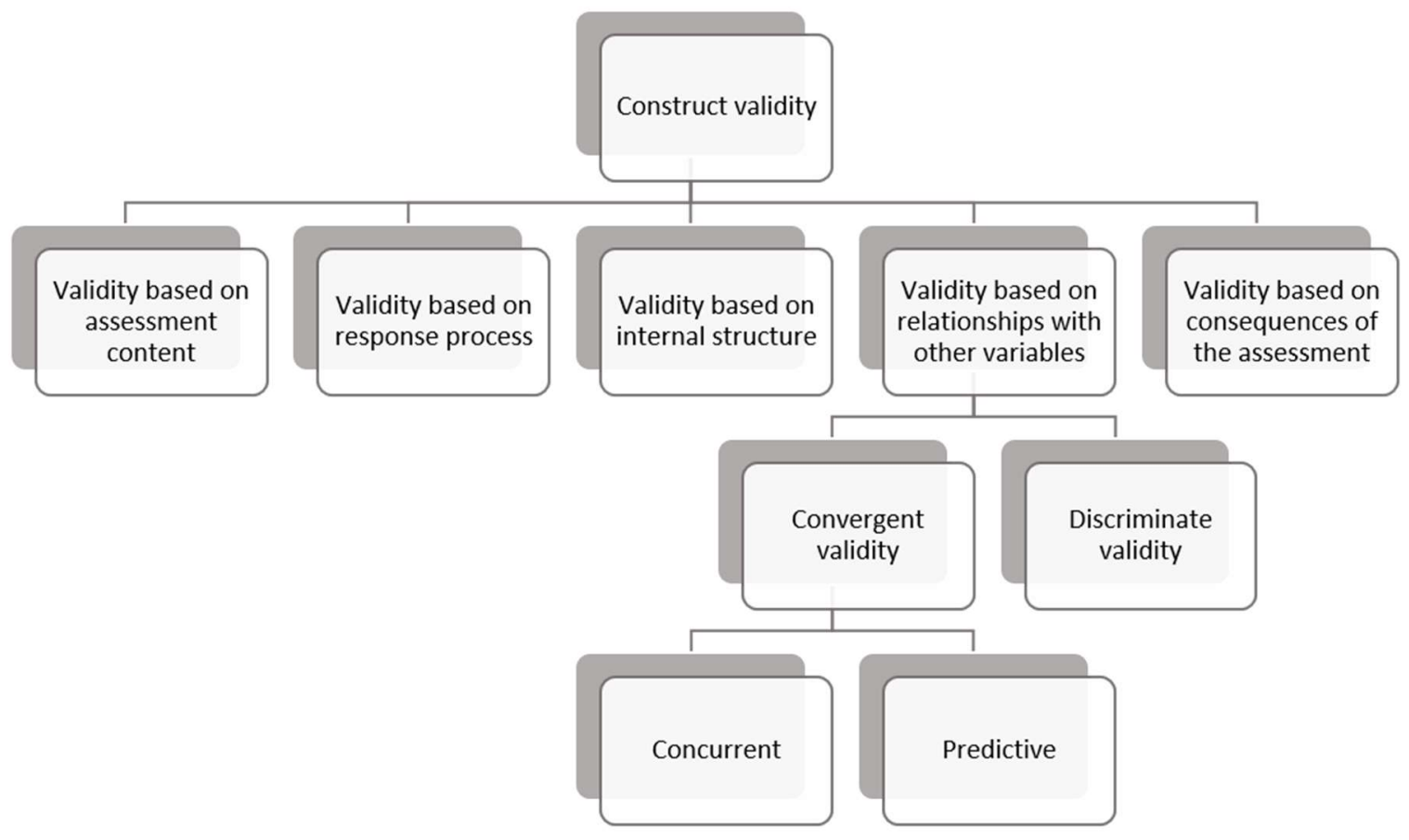

Figure 2.1 A snapshot of the contemporary view of validity.

\section{Validity Evidence Based on Assessment Content}

An instrument designed to measure a psychological construct should contain

items that are reflective of that certain construct. If the construct is latent, meaning that it cannot be directly measured, the theory behind the latent construct should dictate what items are on the instrument. The items on the instrument should capture a well-rounded view, meaning that each item should capture a unique facet of the construct. This type of validity is sometimes known as content validity (Furr \& Bacharach, 2008).

There are two main threats to content validity (Furr \& Bacharach, 2008, pp. 172173). The first involves construct-irrelevant items. If construct-irrelevant items are present on the instrument, the validity of the interpretation of the data would be threatened, as the instrument would not be measuring a single construct. For example, if 
an instrument designed to measure IQ asked the respondent to agree or disagree with the item "My favorite color is blue". If this item was included in a total score for IQ, it would not make interpretable sense. The second threat to content validity is construct underrepresentation. That is, if the items on an instrument capture a too-narrow view of the construct, the entirety of the construct is not being measured and therefore the interpretation of the data will not provide a complete picture of the construct. However, instrument developers face a challenge when creating items to wholly capture a construct, as there is a trade-off between construct coverage and realistic assessment conditions. If there are too many items on an assessment, respondents may experience fatigue and may not put much thought into items that occur after fatigue has set in. To help with instrument development and content validity, experts of the particular construct of interest should be consulted to ensure that items are relevant and capture enough of the construct to represent a well-rounded view.

Another aspect of content validity is known as face validity (Furr \& Bacharach, 2008, pp. 173-174). The instrument must appear to be measuring the construct of interest according to both experts of the construct and by non-experts, such as the participants of a study. If a participant's IQ is being measured and there is an item relating to an individual's favorite meal, the validity of the instrument may be questioned by both the participant and an expert in this field. Face validity from the non-expert point of view is not necessarily considered a crucial psychometric piece of validity but can affect the data provided by the instrument. 


\section{Validity Evidence Based on Response Processes}

When an instrument is distributed to a target sample, it is presumed that

respondents go through a psychological process that includes four steps when responding in an optimal manner (Krosnick \& Presser, 2010). First, a respondent must read and interpret the intent of the item. Second, they must access and connect relevant memories. Third, they need to organize these memories into a single judgement. Lastly, they need to convert this judgement into one of the responses on the response scale of the instrument and select the most appropriate choice. This process can provide respondents with a high cognitive load. Therefore, items should be designed in a way to provide the least amount of cognitive load and the instrument should provide clear instructions on how to respond to the items so they are less likely to be misinterpreted. If respondents interpret items in different ways, this threatens the response processes validity of the instrument, as responses will have different meanings for individuals and this will muddy the interpretation of the data. To provide evidence of response process validity, cognitive interviews are often conducted (Arjoon, Xu, \& Lewis, 2013; Willis, 2005).

\section{Validity Evidence Based on the Internal Structure}

Validity evidence based on the internal structure of an instrument involves providing evidence that data from the items on an instrument are structured the way they were intended (Furr \& Bacharach, 2008, pp. 174-177). If the instrument was designed to capture a single latent construct based on theory, there needs to be evidence that the structure of the instrument data is indeed unidimensional. Additionally, there are instruments designed to capture multiple related latent constructs or sub-constructs of a single latent construct. Data from these instruments need to have evidence of 
multidimensionality, with item data organized in their respective constructs or subconstructs. Dimensionality also has an influence on the way data is interpreted. If an instrument measures multiple dimensions, each dimension could be totaled and averaged to create a score for each individual dimension. It is inappropriate to total and average scores across dimensions if the instrument is shown to in fact be multidimensional.

The most common way to provide evidence of uni- or multidimensionality is through methods of factor analysis. The goal of factor analytic methods are to reveal the relations between the indicator variables (item responses) and their corresponding constructs (Furr \& Bacharach, 2008). The strength of the relation between an observed variable and the corresponding construct is represented by its factor loading; a value that ranges from 0 to 1 . The square of this value indicates how much variance within the indicator variable is explained by the construct. For example, if an indicator variable has a factor loading of 0.80 , this underlying construct explains $64 \%$ of the variance within that indicator variable. The variance unexplained by the construct is attributed to systematic and random error.

There are two types of factor analysis: exploratory and confirmatory. Exploratory factor analysis (EFA) is used to investigate how many underlying constructs are present within a set of indicator variables. EFA is traditionally used in the early stages of instrument development to investigate whether data from the indicator items, created to provide information about specified constructs, are loading on the correct number of factors. EFA uses a similar process to principle component analysis (PCA) but it is theoretically different (Cai, 2013; Preacher \& MacCallum, 2003). PCA is commonly used 
as a data reduction technique that assumes no measurement error and therefore uses weighted composites to separate indicator variables into "components". EFA does assume measurement error and estimates measurement error for each indicator in addition to separating indicator variables into "factors". EFA can provide information about each indicators' relation with underlying constructs but there is no specified $a$ priori model assigned and therefore, EFA does not provide useful global model fit information. To obtain this type of information, confirmatory factor analysis (CFA) is used. CFA provides information about the fit of a hypothesized model to the collected data (Brown, 2014, p. 40). This method is commonly used after EFA has been performed on a previously collected sample in order to confirm the structure of the assessment within a population. CFA is an incredibly valuable tool in providing validity evidence to support the internal structure of an assessment across differing populations once a model has been established. It is important to recognize that the terms exploratory and confirmatory should not necessarily be taken at face value in relation to the factor analysis methods they describe (Kline, 2016, p. 197). EFA can be used as a more confirmatory method by specifying the number of factors during the analysis and confirming that the indicator items show high loadings on their intended factors while CFA can be used as a more exploratory method by comparing multiple hypothesized models. Both EFA and CFA are commonly used in instrument development and either can help to provide validity support for the internal structure of an assessment within a specific population. 


\section{Validity Evidence Based on Relations of the Assessment with Other Variables}

As stated previously, instruments are created with guidance from a theoretical

framework to measure a construct or constructs. Relations between constructs described by the theoretical framework of interest should hold true when operationalized to a measurement. Providing evidence that the theorized relations and the actual relations provided by the instrument are in alignment provides support for validity based on relations of the assessment with other variables (Furr \& Bacharach, 2008, pp. 179-182).

There are two types of validity evidence based on relations of the assessment with other variables; convergent and discriminant. Convergent validity evidence is provided by demonstrating that the measures of two related constructs are correlated. Convergent validity encompasses both predictive and concurrent validity as well. Predictive validity is supported when variables that are theorized to predict other variables are shown to in fact be predictive. For example, self-efficacy at the beginning of a course predicting success in that particular course. For validity evidence to qualify as predictive, the variables need to be collected successively. Concurrent validity is similar to predictive validity; however, the measured variables are collected concurrently. For example, selfefficacy measured at the end of a course predicting the final exam grade. Discriminant validity evidence is supported by demonstrating that two variables that are theorized to have no relation are in fact not correlated to one another. For example, intelligence should not be highly correlated with an individual's favorite color.

\section{Validity Evidence Based on Consequences of the Assessment}

This last facet of validity pertains to the practical use of an instrument and specifically the consequences of its use. There should be no bias against specific groups 
when an attribute is being measured among multiple groups. For example, if females are assessed on job performance and score lower than their male counterparts on average, it needs to be shown that this is not an artifact of the instrument itself. While the consequences of instrument use are important to consider, it is debated whether or not this should be considered a facet of validity (Furr \& Bacharach, 2008, p. 182). It is not commonly considered in chemistry education research but is stated by the Standards for Educational and Psychological Testing as a facet of validity (American Educational Research Association, American Psychological Association, \& National Council on Measurement in Education, 2014).

\section{Reliability}

It is a simple task to calculate reliability, or precision, when using a UV-Vis spectrophotometer to measure the absorbance of a sample. To do this, the measurement would be repeated multiple times and the standard deviation of the data calculated. If the standard deviation value falls within an acceptable range, there is evidence that the UVVis spectrophotometer is providing reliable data. This becomes much more complicated when assessing the reliability of data obtained from measuring a psychological attribute within a human sample. Psychological attributes are commonly measured using instruments containing self-report items. If we follow the UV-Vis spectrophotometer example, these items would be given to a person multiple times and we would hope they respond in the same way each time. But what if the participant responds in the same way simply because they remember their responses from the previous time? Or what if the responses change because the psychological attribute is not stable? What if the participant becomes bored and stops taking the responses seriously? This is where the analogy of 
reliability to precision falls apart. While reliability of a psychological instrument still pertains to random error, it is not handled in the same way as precision of a chemical instrument (Komperda, Pentecost, \& Barbera, 2018).

To discuss reliability pertaining to a psychological instrument, it is necessary to introduce classical test theory (CTT) (Novick, 1966). When a psychological attribute is measured by an item, the score of that item is known as an observed score. One cannot know the true value of an attribute, such as intelligence, and therefore error will always be associated with an observed score. This is reflected in CTT by Equation 2.1.

$$
\text { Observed Score }=\text { True Score }+ \text { Error }
$$

The purpose of reliability is to determine the amount of random error contributing to the overall measurement error. Reliability values have to be estimated. They are not true values because the true score will never be known. Note that reliability is not a measure of an instrument itself but instead a characteristic of the data. The consequence of this statement is that reliability needs to be determined every time the instrument is used. Another important characteristic of reliability is that it does not take dimensionality into account. If an instrument is multi-dimensional, reliability needs to be calculated for every dimension (American Educational Research Association et al., 2014).

There are at least three different methods for estimating reliability within CTT (Furr \& Bacharach, 2008): alternate forms, test-retest, and internal consistency. Internal consistency reliability estimates are the most commonly reported in the field of psychology as they only require a single administration of an instrument (Furr \& Bacharach, 2008, p. 111; Henson, 2001). While there are multiple ways to estimate 
reliability, it is important to choose the estimate that is the most appropriate given the associated assumptions.

The alternate forms reliability estimate (or parallel forms reliability) assumes that two instruments can be designed in a parallel manner. That is, they measure the same true scores of a construct but with different items and have the same amount of error variance. The scores from the two parallel instruments are correlated and the correlation value provides one interpretation of the reliability of the scores. Although theoretically sound, there are multiple issues with alternate forms reliability. It is impossible to know whether the forms are truly parallel and measure the same true scores. Alternate forms of an instrument are sometimes administered during one administration (e.g., version A and version B of a test). According to CTT, error scores from one test are not correlated with error scores on another test so if the instruments are administered at the same time, this could confound the error occurring within each instrument and the assessments would not be truly parallel (Furr \& Bacharach, 2008, p. 106). While it cannot be proven that instruments are parallel, there can be instruments that fit the assumptions of parallel assessments. If this is the case, the alternate forms reliability estimate could be deemed appropriate.

The test-retest reliability estimate avoids the requirement of designing parallel instruments. Within test-retest, the same instrument would be administered to the same sample on different occasions. The correlation between the scores would provide an interpretation of reliability. For this to be true, there are two assumptions that underlie test-retest reliability, the construct of interest must remain stable between the two 
administrations and the error variance of the tests must be equal. The first assumption highlights the requirement of a psychological construct that would remain stable between test administrations. Even with a stable construct, there can be variability between scores of the separate administrations. One issue that threatens test-retest reliability is the timing between test administrations (Furr \& Bacharach, 2008, p. 109). If the span between administrations is too long, it is possible for the psychological construct to change. For example, if the construct of interest was depression and a respondent started therapy in between administrations, that construct may not be considered a stable state over that time. If the span between administrations is too short, carryover effects can occur where participants may simply remember and re-record their responses from the previous administration. If the assumption of equal error variance is met, there is confidence that the psychological construct of interest is stable within the retest period, and carryover effects are not occurring between administrations, test-retest reliability may be an appropriate interpretation of reliability.

Internal consistency reliability estimates are used for the purpose of demonstrating that a group of items measure a single psychological attribute. While alternate forms and test-retest have assumptions that can limit their practicality, internal consistency reliability estimates may be more practical for a wide variety of data. Additionally, while the two previous forms of reliability consist of correlations between two independent scores, internal consistency is comprised of multiple statistics to choose from. While there are multiple computations of internal consistency, they all operate under the assumption that a group of items administered once and designed to measure a 
single construct can be split to create parallel sub-forms. One view of internal consistency relies on split-half estimates. These estimates operate under the assumption that a group of items can be split in half to create two parallel sub-forms. The correlation of these two forms is calculated and then used in a formula to compute a reliability estimate. There are multiple split-half reliability formulas available including the Spearman-Brown formula, the Spearman-Brown prophecy formula, and the Spearman-Brown split-half formula (Furr \& Bacharach, 2008, p. 112). A downfall of split-half reliability estimates is that, similar to alternate forms reliability, they rely on the assumption that the sub-forms are parallel. Cronbach's coefficient alpha and the Kuder-Richardson 20 formula $\left(\mathrm{KR}_{20}\right)$ are estimates of internal consistency reliability at the item-level (Cronbach, 1951; Kuder \& Richardson, 1937). Alpha is used with continuous items while $\mathrm{KR}_{20}$ is used with binary items, and is a special case of Cronbach's alpha. Alpha is the most common way that reliability is reported (Furr \& Bacharach, 2008, p. 115). It is calculated by first finding the variance of scores on the entire instrument as well as the covariances between each set of items (pairwise covariances). These values are then placed in the coefficient alpha formula to provide an estimate of reliability. The $\mathrm{KR}_{20}$ formula is a simpler computational formula for binary items that is algebraically equivalent to alpha (Furr \& Bacharach, 2008, p. 119).

While alpha is the most commonly calculated measure of reliability, it is often used inappropriately (Komperda, Pentecost, et al., 2018; Sijtsma, 2008). An important assumption for alpha that is often ignored lies within a factor analysis framework. Alpha has the underlying assumption that the data fits a parallel or tau-equivalent model. A 
parallel model assumes identical loadings and error terms for a group of items that measure a single construct. A tau-equivalent model relaxes this assumption slightly and assumes identical loadings with varied error terms. These assumptions oftentimes do not hold up in practice (Komperda, Pentecost, et al., 2018). There is an alternate measure to alpha called omega (McDonald, 1999, p. 89). Omega is equivalent to alpha for parallel and tau-equivalent models but can also function under the assumption of a congeneric model, which is most often prevalent in practice. A congeneric model has the most relaxed assumptions, allowing for varied loadings as well as varied error terms within a set of items. While alpha can be appropriate when the required assumptions are met, it is crucial to test those assumptions before choosing the appropriate reliability estimate to report.

\section{Existing Science and Discipline-Specific Identity Scales and Measures}

To support construct validity of a measure, the measure needs to be

operationalized specifically for its intended use. There are existing science and disciplinespecific identity measures within the education literature, however, they have been operationalized to roles other than "science" or "chemistry person" such as scientist identity (Chemers et al., 2011; Estrada et al., 2011) and science student identity (Stets et al., 2017) or have not been operationalized specifically to chemistry(Cass et al., 2011; Godwin \& Potvin, 2013; Hazari et al., 2010). Brickhouse and colleagues (2000) have stressed the importance of studying appropriate communities of practice in which science identities can form. For example, the term scientist could pose a narrow view of what it means to engage in science as it implies that an individual is actively engaging in science outside of the classroom. Studying a research scientist community of practice (e.g., 
scientist rather than science student or science person) in relation to the formation of students' science identity could be problematic as it could be irrelevant to students depending on their experience with science outside of school. While the Brickhouse and colleagues study was performed with middle school students, the issue of studying science identity formation in the context of a research scientist community of practice could pose the same problems with college students, especially in the beginning of their undergraduate career when students may have zero research experience. Measures of research scientist identity have been used to study the impacts of science support experiences such as research and mentoring that occur outside of the classroom (Chemers et al., 2011; Estrada, Hernandez, \& Schultz, 2018; Robnett, Chemers, \& Zurbriggen, 2015) but may not be appropriate for identity changes within the classroom. There is one measure of science identity designed to measure the impact that a specific intervention has on identity within a course (Childers \& Jones, 2017) but this science identity measure is designed to addresses one intervention and is therefore useful for very specific intervention assessment. Although science and discipline-specific identity measures currently exist, many lack the appropriate context or are not designed to specifically measure chemistry identity.

The most widely used identity measure has been based off of the physics identity framework (Hazari et al., 2010). In addition to physics, these measures have been operationalized to math (Cribbs et al., 2015), science (Godwin, Potvin, \& Hazari, 2013) as well as combined to measure engineering identity (Godwin, Potvin, Hazari, \& Lock, 2013).These measures have been used to explore topics such as gender differences in 
both the impacts of high school pedagogy on physics (Hazari et al., 2010) as well as the impacts of physics identity on career choice (Lock, Castillo, Hazari, \& Potvin, 2015). Portions of the scale have been used to study the importance of recognition in science identity formation in women (Hazari, Brewe, Goertzen, \& Hodapp, 2017). The measure has also been used to study choice of engineering based on math-identity (Cass et al., 2011; Godwin, Potvin, Hazari, \& Lock, 2016).

The first measure of the physics identity framework, consisting of the subconstructs of performance/competence, recognition, and interest was first tested by to measure the experiences of high school physics courses and career outcome expectations on physics identity (Hazari et al., 2010). This instrument was distributed to 6,722 undergraduate students in introductory English courses at 24 universities via the Persistence Research in Science and Engineering (PRiSE) project. The PRiSE survey set out to explore factors from high school that influence persistence of females within STEM and was developed using three methods: a literature review of constructs that affect persistence, open-ended responses from 259 secondary science teachers and 153 scientists on what they believe influences persistence in college, and extraction of items from a previously used national survey (Factors Influencing College Success-FICSS). In total, the PRiSE survey was comprised of 50 items across 6 sections, requiring more than 250 individual responses from students. The original physics identity survey was compiled from a range of PRiSE items. However, few details were provided regarding why the items were chosen or how they were assigned to each sub-construct of identity through the theoretical framework of SCCT. 
Performance was measured using five PRiSE items about middle school and high school grades along with SAT scores. It is of note here that the construct of performance was measured using student ability in the form of past grades, whereas the definition of performance within the physics identity framework described performance as belief in ability. Competence was measured using two PRiSE items about students' perceived confidence in middle school math and science. Within SCCT, the construct of performance is theorized to influence one's self-efficacy and therefore career goals and choices while the construct of competence is not specifically mentioned.

Nineteen PRiSE items were used to measure the construct of interest. These items reflected interest in specific tasks associated with three domains: physics interest (e.g., mechanics and electromagnetism), science interest (e.g., understanding natural phenomena and using mathematics), and science activity (e.g., participation in science groups/clubs/camps and science/math competitions). Within SCCT, interest is defined as “...likes, dislikes, and indifferences regarding career-relevant activities and occupations"(Lent et al., 1994, p. 88). The interest items on the PRiSE survey were not explicitly tied to career-relevant activities or occupations.

Items measuring recognition on the PRiSE survey included items where students rated whether certain groups (i.e., their science teacher or their parents/relatives/friends) saw them as a physics person. These two items of recognition were retained for the physics survey, however, the definition of recognition within the physics identity framework pertains to whether a student is recognized as a good "physics student". These wording differences could conflict based on students' definitions of "physics person". 
Recognition is not specifically mentioned in SCCT but does align well with the verbal persuasion source of self-efficacy defined within SCT (Bandura \& National Inst of Mental Health, 1986, pp. 405-406), although this alignment is not mentioned by Hazari and colleagues (2010).

Validity based on the internal structure of the instrument was provided through EFA. The analysis showed that the constructs of performance and competence were not separately distinguished by students and were therefore combined to form a single construct termed 'performance/competence'. Within this study, the items pertaining to each construct were averaged to create a composite score. The composite scores were then correlated to a single physics identity item "Do you see yourself as a physics person?" that was ranked by respondents from $0-5$, with 0 being "No, not at all" to 5 being, "Yes, very much". All composite scores were highly positively correlated.

After the constructs of performance/competence, recognition, and interest were shown to predict physics identity through the PRiSE survey study (Hazari et al., 2010), the items used to measure the sub-constructs of identity were modified. Items from the PRiSE study were no longer used and new items were created for each scale and have been used in multiple studies to measure physics and math identity (Cass et al., 2011; Cribbs et al., 2015; Godwin, Potvin, \& Hazari, 2013; Verdín \& Godwin, 2017). EFA has been performed on these items within each study to show that they indeed load on the three distinct factors of performance/competence, recognition, and interest although there has been no explanation for the change in item modifications for each scale. 
While these measures have gone through extensive use, multiple iterations, and basic psychometric evaluation, the instruments that have been designed based off of the physics identity framework lack validity evidence based on assessment content. The constructs of performance/competence, recognition, and interest are defined in terms of a wider science identity theory (Carlone \& Johnson, 2007; Hazari et al., 2010), but each construct lacks a clear theoretical grounding of the items used to measure them, which is an absolute necessity of instrumentation design (Kline, 2016) . 


\section{Chapter 3}

\section{Methodology}

\section{Research Questions}

The primary goal of this study was to more strongly align an existing disciplinespecific identity instrument with constructs that are prominent in psychological literature. The secondary goal of the project was to operationalize the instrument to science and chemistry identities. The goals of this project were addressed by the following research questions:

Q1. What themes arise when students are asked questions reflecting performance/competence, recognition, and interest, pertaining to science or chemistry, as described by the physics identity framework?

Q2. To what extent do reported themes align with proposed and other affective constructs?

Q3. To what degree will an instrument containing items designed to measure mastery experiences, verbal persuasion, and situational interest show psychometric functionality in undergraduate chemistry courses with

a. science-specific wording?

b. chemistry-specific wording?

Q4. What are the relations between mastery experiences, verbal persuasion, situational interest and a

a. science identity indicator?

b. chemistry identity indicator? 
In order to address these research questions, the study was broken into two phases. The first phase consisted of the first two research questions, where qualitative methods were used to investigate themes within the physics identity framework and to subsequently investigate overlapping affective constructs with established theoretical frameworks. The second phase of the study addressed the final two research questions which were built directly upon the results of the first phase and explains the specificity of research questions three and four.

\section{Phase One: Investigating the Overlap of the Sub-Constructs of the Physics Identity Framework With Established Affective Constructs.}

The purpose of this phase was to address research questions one and two; what themes arise when students are asked questions reflecting the physics identity framework (Hazari et al., 2010) and do those themes overlap with constructs that have established theoretical frameworks? The constructs outlined by the physics identity framework included performance/competence, recognition, and interest. Performance/competence was defined as the "belief in the ability to perform on physics tasks and the belief in ability to understand physics content." Recognition was defined as "identification from others as being a physics person." The final construct of interest was defined as "desire/curiosity to think about and understand physics." Semi-structured interviews and thematic analysis were used in order to investigate these research questions.

\section{Participants}

Research conducted in this portion of the study was approved under PSU IRB

\#174340. The sample for this study consisted of students enrolled in selected undergraduate chemistry courses Portland State University. To sample a range of student levels (e.g., by major and year in degree), the selected courses included an off-sequence 
general chemistry course for science majors, two sections of an organic chemistry course, and a biochemistry course for non-biochemistry majors. Enrollment for the four courses were $233,162,131$, and 235 , respectively. A final question on a previously administered pilot survey asked students if they were willing to participate in a follow-up interview. Students who responded positively to the question were recruited for interviews. In order to capture a range of responses, quota sampling (Tourangeau \& Yan, 2012) was used, where an equal number of participants per specified responses were selected. Responses to the two identity items, "I see myself as a science person" and "I see myself as a chemistry person" from the previously administered survey, were used to select participants. Students who agreed to participate in an interview were separated into three groups: 1) those who agreed with both statements (by selecting "agree" or "strongly agree" on the Likert scale for both items), 2) those who did not agree with both statements (by selecting "neither agree or disagree", "disagree", or "strongly disagree" for both items), and 3) those with mixed responses across the two identity items. One student from each group was then randomly selected using Google's random number generator for an interview. This was repeated for each course type for a total of nine interviews.

\section{Interview Design and Protocol}

Participants were interviewed in a private office space and audio recorded for transcription purposes. Before audio recording began, participants were given the approved IRB consent form to read and sign and provided with the opportunity to ask any questions about the study prior to consenting. A semi-structured interview format (Smith, 1995) was used to investigate the sub-constructs of the physics identity framework 
(Hazari et al., 2010)contextualized to science and chemistry; performance/competence, recognition, and interest. Items from a survey designed from the physics identity framework (Godwin, Potvin, \& Hazari, 2013) were used as a template for the interviews as these items have been the most widely used to measure performance/competence, recognition, and interest. These items were modified for the purpose of being posed as open-ended questions to students. These open-ended questions formed the semistructured interview protocol (Appendix A). Follow up questions were asked as necessary for clarification of responses. The participants' responses to the semi-structured interview questions were used as data to search for themes and their overlap with established affective constructs.

\section{Interview Analysis}

Interviews were transcribed verbatim and subsequently analyzed using reflexive thematic analysis (Braun, Clarke, Hayfield, \& Terry, 2019) along with guidelines for semi-structured interview analysis (Smith, 1995). Thematic analysis is a method used to describe themes (patterns of meaning) within the data. There are many ways to utilize thematic analysis, but regardless of how it is utilized, it is crucial for researchers to report the way the method was used so that the assumptions of the method are clear (Braun \& Clarke, 2006). The thematic analysis was performed through an essentialist lens (Braun $\&$ Clarke, 2006), which assumes the responses of participants directly reflect their experiences. Reflexive thematic analysis (Braun et al., 2019) was used as an inductive analysis method where themes are derived from the collected data as compared to a deductive analysis, where pre-conceived themes are applied to the data (Hesse-Biber, 2017, pp. 11-12). Inductive analysis was chosen in order to extract all possible themes 
from the data. While inductive analysis assumes that there is no preconceived hypothesis when analyzing the data, it does not assume that the researcher is approaching the data as a "blank slate"(Braun et al., 2019). It simply means that the researcher is starting the analysis with the data rather than searching for specific hypotheses within the data (Braun \& Clarke, 2006; Braun et al., 2019). In addition to inductive coding, coding was carried out in a reflexive manner, where themes arise out of meaning-based patterns within codes. Instead of "accurately" summarizing the data, as methods that use inter-raterreliability aim to do, reflexive analysis aims to provide a compelling interpretation of the patterns within the data, grounded in the data (Braun et al., 2019). All coding was carried out through the software MAXQDA (Version 18.2.0).

The process of data analysis was carried out both independently and collaboratively by the first author and an undergraduate researcher. The reflexive thematic analysis used in this study followed five steps as described by Braun and colleagues (2019): 1) familiarization, 2) generating codes, 3) constructing themes, 4) revising themes, and 5) defining themes. First, two of the nine transcripts were read independently and multiple times to gain familiarity with the data. During these reads, each researcher recorded notes on patterns within the two transcripts. The second step contained multiple sub-steps. The two researchers came together to record all identified patterns from their notes and combined similar patterns when necessary. A codebook with codes and definitions was then created based on the final list of patterns. This codebook was then used by the researchers to independently code two additional transcripts and notes of new codes were recorded. Once again, the researchers came 
together to refine codes and edit the codebook. This process was repeated one more time with two additional transcripts until a final codebook was established. Reflexive thematic analysis does not use strict measures of inter-rater reliability. Coding is considered an iterative process where codes are continually developed throughout analysis(Braun et al., 2019). In this case, the codebook was considered complete when the two researchers reached a consensus that there were no more unique codes. The final codebook was used by the researchers to independently code two new transcripts to confirm that no new codes were discovered. Once the final codebook was confirmed, the last transcript was coded and all transcripts were re-coded to consensus. After all transcripts were coded, the final three steps of analysis consisted of evaluating the generated codes for themes, revising, and defining codes. Codes were rearranged into groups multiple times until the first author decided on a final set of groupings that resulted in themes. The themes were then defined by the first author, reviewed by the second author, and subsequently discussed with secondary researchers. The final themes were used to explore connections of the sub-constructs of performance/competence, recognition, and interest with established affective constructs.

Phase Two: Testing the Psychometric Properties of and Relations Between Scales of Mastery Experience, Verbal Persuasion, and Situational Interest in Undergraduate Chemistry Courses.

Phase two directly built upon the results of phase one. The purpose of phase two of this study was to address research questions three and four; to what degree will a measure containing the constructs of mastery experiences, verbal persuasion, and situational interest show psychometric functionality in undergraduate chemistry courses with both science- and chemistry-specific worded versions and what are the relations 
between the constructs? Scales from previously used measures were revised to fit the context of a science and chemistry undergraduate setting and distributed in two different chemistry course types. A variety of quantitative methods were then used to analyze the validity and reliability of the data produced by the instrument. After investigating the psychometric properties of the scales, the relations between the constructs of science and chemistry identity were modeled and tested using structural equation modeling (SEM).

\section{Scales}

The scales chosen for the study were not all originally developed to measure constructs in a science- or chemistry-specific context, therefore, scale items were modified to include the context of science and chemistry undergraduate courses. The constructs that these scales measure have been hypothesized to influence science and chemistry identity. The specific scales were designed to measure the constructs of mastery experiences, verbal persuasion, and situational interest.

\section{Mastery Experience and Verbal Persuasion Scales}

The mastery experience and verbal persuasion scales were taken from the Sources of Middle School Mathematics Self-Efficacy Scale (Usher \& Pajares, 2009). The instrument was developed in response to the lack of a measure targeted toward the four sources of self-efficacy (mastery experiences, verbal persuasion, vicarious experiences, and physiological state) in middle school mathematics. Items for these sources of were developed according to Bandura's social cognitive theory (SCT) (1986), which encompasses the theory of self-efficacy. Scales aligned with these sources were iteratively developed over three rounds of data collection and psychometric analysis. A Likert-scale is a five-point response scale that ranges from strongly disagree to strongly 
agree and also contains a "neutral" response. Any scale similar to this but modified is known as a Liker-type response scale. Each finalized scale contained six items and were on a six-point Likert-type response scale ranging from definitely false to definitely true.

While thorough psychometric analysis was performed on the Sources of Middle School Mathematics Self-Efficacy scale in the context of middle school students in Grades 6-8, the authors state that next steps would be to test the measure across different populations and domains (Usher \& Pajares, 2009). Although the measure was tailored in wording for middle school students, the theory was rooted in SCT (measure reviewed by Bandura himself) and items on the measure mirrored already existing sources of selfefficacy measures developed in a college setting (Fencl \& Scheel, 2003; Lent, Lopez, Brown, \& Gore, 1996). For this reason, as well as the support provided by the psychometric analysis performed on the measure, the social persuasion and mastery experience subscales from the Sources of Middle School Mathematics Self-Efficacy scale were chosen.

\section{Situational Interest Scales}

The initial and maintained situational interest scales developed by Ferrell and Barbera (2015) were used in this study. The two measures were originally operationalized in psychology (Harackiewicz, Durik, Barron, Linnenbrink-Garcia, \& Tauer, 2008) and adapted to a General Chemistry context (Ferrell \& Barbera, 2015). Both initial and maintained interest measures were chosen in order to capture the interest of students at the beginning of a course and to measure the "hold" portion of situational interest (Linnenbrink-Garcia et al., 2010), respectively. 
Both measures contained items from two constructs: feeling- and value-related interest (Schiefele, 1999). Feeling-related interest items on the initial interest scale were tied to emotional arousal, for example, "I am excited about taking this class". Valuerelated items were tied to importance/utility, for example, "I think the field of chemistry is an important discipline". The initial interest measure contained seven items; four feeling- and three value-related interest items. The maintained interest measure contained eight items; four feeling- and four value-related interest items. All items were on fivepoint Likert-scale ranging from strongly disagree to strongly agree with a "neutral" response.

\section{Preliminary Wording and Scale Changes}

All scales were revised to created separate "science" and "chemistry" versions. Items that were revised to contain the phrase, "this class" were included in both science and chemistry versions during analysis. Wording changes were made to each scale, as needed, to reflect the domains of science and chemistry, specifically in an undergraduate course setting. The mastery experience and verbal persuasion scales were originally operationalized for middle school mathematics. To modify these items, the word "math" was modified to "science" and "chemistry", for example, "Even when I study very hard, I do poorly in math" was revised to "Even when I study very hard, I do poorly in science" and "Even when I study very hard, I do poorly in chemistry". Additionally, items containing words in the context of middle school were changed to reflect undergraduate courses. For example, the word "tests" was modified to "exams". The situational interest scales were originally operationalized specifically for General Chemistry; therefore, for the purpose of using the scale in multiple undergraduate chemistry courses, items were 
modified to remove this specificity. For example, the item "I think that what we will study in General Chemistry will be important for me to know" was modified to "I think that what we will study in this class will be important for me to know". Phase two original and revised survey items are included in Appendix B.

The mastery experience and verbal persuasion response scales were modified from a six-point Likert-type scale to a five-point Likert scale to align with the situational interest scales and for the purpose of including a neutral mid-point within the scale. The authors of the Sources of Middle School Mathematics Self-Efficacy Scale did not give an argument for the number of response choices on their Likert-type scale. It is unadvisable to include a mid-point in a Likert-scale for measures that contain items that could be socially undesirable because participants may select a neutral option to avoid responding honestly to the item (Johns, 2005). The mastery experience and verbal persuasion scales do not have items that could be deemed socially undesirable and therefore a mid-point was included to capture participants who had a legitimate neutral response to the items. Additionally, the change in wording of the Likert-type response scale (definitely false to definitely true) to the Likert response scale (strongly agree to strongly disagree) did not change the meaning of any of the items.

\section{Participants}

Research conducted in this portion of the study was approved under PSU IRB \#184548. The sample for this study consisted of students in undergraduate chemistry courses at three Northwestern universities, a Southwestern university, and two Midwestern universities. Convenience sampling (Tourangeau \& Yan, 2012, p. 229) was used for this study. Students enrolled in specific courses, volunteered to participate in the 
study. To sample a range of student levels (e.g., by major and year in degree), the selected courses included organic and general chemistry courses targeted to science majors. A total of 855 organic and 2,324 general chemistry students were recruited for participation in the survey. Nominal extra credit was offered in all courses according to the approved IRB.

\section{Survey Data Collection}

Survey distribution occurred during the first and last weeks of the courses during Fall 2018. Students were recruited by the primary researcher through an in-class announcement delivered either through a recorded video or in person. The survey was hosted on the website Qualtrics and the link to the survey was posted to the respective course websites after each in-class announcement. The survey links lead students to the consent form for the study where they could either enter their identifying information and either accept or decline participation. Students who completed the Informed Consent page and either declined or agreed to participate obtained extra credit in the courses. The first survey (time 1) contained the mastery experiences, verbal persuasion, initial interest, and the additional identity indicator (i.e., self-recognition). In the last survey (time 2), the initial interest scale was replaced with the maintained interest scale. Both "science" and “chemistry" versions of each scale and identity indicator were included on the survey with a five-point Likert scale at both time points. Each "science" item was immediately followed by its "chemistry" item counterpart excluding items that asked about "this class". This was done so that students would directly compare their responses for the chemistry and science wording of the survey. Each of the pairs of items were randomized for each student to avoid any order effects that could be present. 


\section{Survey Data Analysis}

Data Cleaning

Only students who completed both time 1 and time 2 measures were included in the dataset. A self-check item was included in the survey that read "Please select 'Disagree' for this item'. Students who selected a response other than 'Disagree' were removed from the dataset. Missing data was deleted based on wording type responses. For example, if a student had completed all of the science-worded items at times 1 and 2, but did not complete an item within the chemistry wording at time 1 , their chemistry responses from both times 1 and 2 were removed and science responses retained.

Responses from the time 1 and time 2 measures were then matched. After matching complete datasets, duplicated responses were removed based on the self-reported name of the respondents. If duplicated responses were present, the first entry was retained. Descriptive Statistics

Descriptive statistics were computed using the open source software R (Version

3.4.4). Means and standard deviations of all items were analyzed to assess the central tendency and variability of the data (Allen \& Yen, 2001, pp. 18-19). Correlation coefficients were calculated to observe the initial strength of item relations (Allen \& Yen, 2001, pp. 23-31) within the science and chemistry worded versions of the survey. Skew and kurtosis were analyzed to assess the data for non-normality. Although there is no universal consensus on cutoffs for the degree of non-normality, data was described as non-normal if skew and kurtosis values -1 and 1(Huck, 2012). Analyzing data for skew and kurtosis is critical as it provides information on whether non-normal data corrections are needed in further statistical analysis. Reliability was provided for each scale with the reliability estimate omega (Komperda, Pentecost, et al., 2018). 


\section{Confirmatory Factor Analysis}

In order to make meaningful comparisons between groups, evidence must be

presented to show that the measure used for comparison is functioning in the same way in every context that is to be compared. For example, the internal structure of the instrument must be the same when measuring multiple groups if comparisons are to be made i.e., the instrument is measuring the same construct in both groups. Factor analysis is a group of methods commonly used to assess the structure of an instrument. Confirmatory factor analysis (CFA) is used to assess how well a proposed model of latent factors fits observed data as compared to Exploratory Factor Analysis (EFA) which is used to discover the number of factors and how observed variables are organized into latent factors (Kline, 2016, pp. 190-191). CFA was chosen over EFA because the scales used in this study were based on a strong a priori hypothesis for the factor structure of each scale. CFA was used to assess validity of the data based on the internal structure of the science and chemistry worded versions of the scales in both general and organic chemistry. These analyses were conducted using the lavaan package (Version 0.523.1097) for $\mathrm{R}$ (Version 3.4.4). The scales of mastery experiences, verbal persuasion, and the two constructs of initial interest (value- and feeling- related interest) measured at time 1 were each considered separate latent variables (or factors). CFAs were conducted in multiple steps, building up to the a priori correlated multi-factor model. Two-factor CFAs were run, initial feeling- with initial value-related interest, and mastery experiences with verbal persuasion, in order to mimic previous analyses (Ferrell \& Barbera, 2015; Usher \& Pajares, 2009), before combining all of the scales into a multi-factor model. Note that item error and factor variances are omitted in the models for clarity. All models 
were tested separately for the chemistry and science wording with initial interest items containing "this class" contained in both model versions. CFAs were conducted separately for each course type. To confirm the structure of the instrument at both time points, this was repeated for time 2 scales, with maintained interest replacing initial interest.

Multiple estimators are available for CFA based on the characteristics of the data (e.g., continuous, categorical, normally or non-normally distributed). Continuous data is an assumption of maximum likelihood estimators and while the Likert-scale technically provides ordinal data, the scale can be thought of as continuous when it contains five or more response options and is approximately normally distributed (Dolan, 1994). There is also evidence that non-normal ordinal data can be treated as continuous with robust-error corrections but is not always appropriate as kurtosis increases (Finney \& DiStefano, 2006; Rhemtulla, Brosseau-Liard, \& Savalei, 2012). When data met the appropriate ranges for skew and kurtosis based on the descriptive statistics, the maximum likelihood estimator with the Satorra-Bentler adjustments and robust standard error corrections (MLM) (Satorra \& Bentler, 1988) was the first estimator considered, as it is recommended over ordinary maximum likelihood (ML) for moderate nonnormality coupled with small sample sizes (Yu \& Muthén, 2002) and the chi-square statistic simplifies to the ML estimates under multivariate normality (Curran, West, \& Finch, 1996).

In order to determine if there was acceptable global model fit to the data, a combination of fit indices were used. There are three common types of fit indices: 
absolute, incremental (or relative), and parsimonious (Kline, 2016, pp. 266-267).

Absolute fit indices measure how well the proposed model fits the data with no other point of reference. Incremental fit indices compare the proposed model to a baseline model and assess the relative improvement in fit. Parsimonious models are indices that provide a "penalty" for model complexity, as the most parsimonious model is desired. Although there is no one consensus to which model-fit statistics should be reported, it is recommended to use a combination of fit indices to assess model fit (Hu \& Bentler, 1999; Schermelleh-Engel, Moosbrugger, \& Müller, 2003). Reported indices within this study include chi-square with its degrees of freedom and $p$ value, the standardized root mean squared residual (SRMR), the root mean squared error of approximation (RMSEA) and its $90 \%$ confidence interval, and the comparative fit index (CFI). These indices were chosen based on guidelines by Kline (2016, pg. 269). The chi-square test statistic was the original model fit statistic and is a measure of how well the population covariance matrix is reproduced by the model. A nonsignificant chi-square indicates that there is no significant difference between the covariance matrix of the population and model (Kline, 2016). Chi-square along with its degrees of freedom and $p$ value are usually reported for "historical significance” (Mueller \& Hancock, 2008) and was not used to solely assess model fit due to its sensitivity to sample size (Schermelleh-Engel et al., 2003). The SRMR is an absolute fit statistic that is a "badness-of-fit" statistic. It is the standardized version of the root mean square residual (RMR), which is a measure of the mean covariance residual with a value of 0 indicating perfect model fit and poorer fit as values increase (Kline, 2016, p. 277). The RMSEA (Steiger, 1990) has been labeled both an 
absolute (Hu \& Bentler, 1999; Kline, 2016, p. 273) and parsimonious fit statistic (Mueller \& Hancock, 2008). It is another "badness-of-fit" statistic and is a measure of how well the population matrix is reproduced by the model matrix, while accounting for complexity of the model with a value of 0 indicating perfect model fit and poorer fit as values increase. The CFI (Bentler, 1990) is an incremental "goodness-of-fit" statistic that compares closeness of fit between the model and a null model. This statistic ranges from 0 to 1 with values closest to 1 indicating better model fit. All of the reported statistics should be within an assigned cutoff range to indicate good model fit.

There is no consensus on absolute cutoff values for global-model fit statistics. The most commonly used recommendations are from $\mathrm{Hu}$ and Bentler (1999) with the use of the ordinary ML estimator. They recommend cutoff values near $0.95,0.06$, and 0.08 for the CFI, RMSEA, and SRMR respectively. Values of 0.95 and 0.05 for the CFI and RMSEA have been recommended for uses specifically with MLM (Yu \& Muthén, 2002) and are similar to the cutoffs recommended by $\mathrm{Hu}$ and Bentler. Joint criteria can also be used to asses model fit such as a SRMR $\leq 0.09$ and $\mathrm{CFI} \geq 0.96$ or RMSEA $\leq 0.06$ (Mueller \& Hancock, 2008). When the MLM estimator was used in this study, values of 0.95, 0.06, and 0.08 were set as the cutoff criteria for the CFI, RMSEA, and SRMR, respectively. If the values did not fit this criterion, the joint criteria suggested by Mueller and Hancock were taken into consideration. Studies of WLSMV have indicated that global fit statistics can be inflated, especially with less than four response categories (Hutchinson \& Olmos, 1998). Values of 0.95 and 0.05 were used as cutoff values for the CFI and RMSEA, respectively when the WLSMV estimator was used. 
If a model does not fit the data based on the defined cutoff criteria for an estimator, the model may be re-specified based on localized fit parameters. As stated by Kline (2016), “...respecification in CFA should be guided as much as possible by substantive considerations; otherwise, respecification could put the researcher in the same situation as the sailor in this adage attributed to Leonardo da Vinci: One who loves practice without theory is like a sailor who boards a ship without a rudder and compass and never knows where he or she may be cast (pgs. 309-310).” Re-specification should be done cautiously and guided by theory as to not over-fit the model to the specific data collected because this can lead to the model being more indicative of the characteristics of the specific sample versus the more general population (Kline, 2016, p. 310). Respecifications such as item modifications or deletions were decided on by inspecting the factor loadings, modification indices, and residuals. Low factor loadings can be indicative of item misfit (Kline, 2016, p. 310). Modification indices (MI) reflect the difference in chi-square of nested models for one degree of freedom based on one parameter change (Brown, 2014). Within the CFA framework, MI usually involve correlations between the residuals of two items, an item and a factor, or two factors if they were not originally allowed to correlate in the specified model. A high MI can indicate items that are similar or items that belong on a different factor. In order to make a change to the model based on the MI, the value should be significant according to the chi-square distribution for the degrees of freedom for the entire model (Hancock, 1999). Residuals were used to assess model misfit. When two items on different factors have large and positive residuals, this can be an indicator that an item is cross-loading on a separate factor (Kline, 2016, p. 
310). These localized statistics were used to assess problematic items. These criteria were then used to guide deletion or modification of items for use in all CFA analyses.

\section{Structural Equation Modeling}

Structural equation modeling (SEM) is a family of related measurement methods that can provide information about causal inference. These methods rely on a prioi models grounded in theory. While SEM is a method of causal inference, it does not provide an absolute model of causal relations between variables. Instead, it can provide support for a theory that is described in the literature and the causal relations described by that theory (Kline, 2016, pp. 9-12).

SEM is similar to path analysis, where causal relations between observed variables are modeled through a series of multiple regressions. The difference between the two techniques is that SEM allows for the study of relations between both observed and latent constructs. Therefore, SEM has both a measurement component of analysis and a structural component of analysis.

A 2-step SEM process was performed, where the measurement component was tested through the CFAs and the structural component was subsequently tested (Mueller \& Hancock, 2008). If there is misfit within a full SEM model, it is hard to decipher whether the misfit is originating from the measurement or structural component. This 2step technique allowed the measurement component of SEM to be analyzed separately from the entire model. If CFA showed that the measurement model was acceptable, any misfit in the full SEM model could be attributed to the structural component of the model. Once the measurement models were analyzed through CFA, the next step was to test longitudinal invariance between time 1 and time 2 measures. 
Before testing the full SEMs, it was necessary to show evidence of longitudinal invariance, i.e., that the repeated measures from times 1 and 2 were measuring the same construct. There are various levels of invariance testing. The lowest level of invariance is configural invariance, where the measures within each group (in this case mastery experiences and verbal persuasion) are shown to be invariant without holding any parameter equal between groups (time 1 and time 2) (Fischer \& Karl, 2019). The next highest level of invariance is metric invariance, where measures are shown to be invariant when the factor loadings are held equal between groups. Higher levels of invariance entail holding additional parameters equal between groups, such as intercepts and latent means. These higher levels of invariance are necessary when comparing any total score of a measure between groups. It is recommended to at least provide evidence of metric invariance between repeated measures before testing a full SEM model (Newsom, 2015). Metric invariance testing was completed for the repeated measures of mastery experience and verbal persuasion for both wording types within each course. Models provided evidence of invariance if the change in chi-square was nonsignificant between configural and metric invariant models (Newsom, 2015). After showing evidence of metric invariance for repeated measures, the full SEMs within Figure 3 were tested for each wording type within each course. To compare SEM parameters between general and organic chemistry courses within each wording type, multi-group metric invariance must be tested (Fischer \& Karl, 2019). Multi-group invariance was determined by a nonsignificant change in chi-square (Cheung \& Rensvold, 2002). 
After invariance testing, full baseline and alternative SEM models (shown in Figure 3.1) were tested. These models were chosen based off of previous models tested with performance/competence, interest, and recognition within the physics identity framework (Cribbs et al., 2015) in order to provide further evidence of overlap between the physics identity framework and the established theoretical frameworks of mastery experiences, situational interest, and verbal persuasion. Each model was tested twice within general and organic chemistry courses; once for the "science" identity wording and once for the "chemistry" identity wording. Items that contain the term "this class" instead of "science" or "chemistry" were included within both models. Individual items and endogenous variable disturbances were not shown in the models (Figure 3.1) for sake of clarity. Also omitted from the figures were the correlations between individual item errors for repeated measures. For example, item 1 on the mastery experience scale from time 1 was correlated with item 1 on the mastery experience scale from time 2 . All SEM analyses were carried out through the lavaan package (Version $0.5-23.1097$ ) in $\mathrm{R}$ (Version 3.4.4). Normality in the data distributions was assessed to determine the appropriate SEM estimator. After estimation, cutoff values indicating good model fit followed that of the CFA models: $\mathrm{CFI} \geq 0.95$, RMSEA $\leq 0.06$, and $\mathrm{SRMR} \leq 0.08$ or joint criteria of SRMR $\leq 0.09$ and $\mathrm{CFI} \geq 0.96$ or RMSEA $\leq 0.06$. 


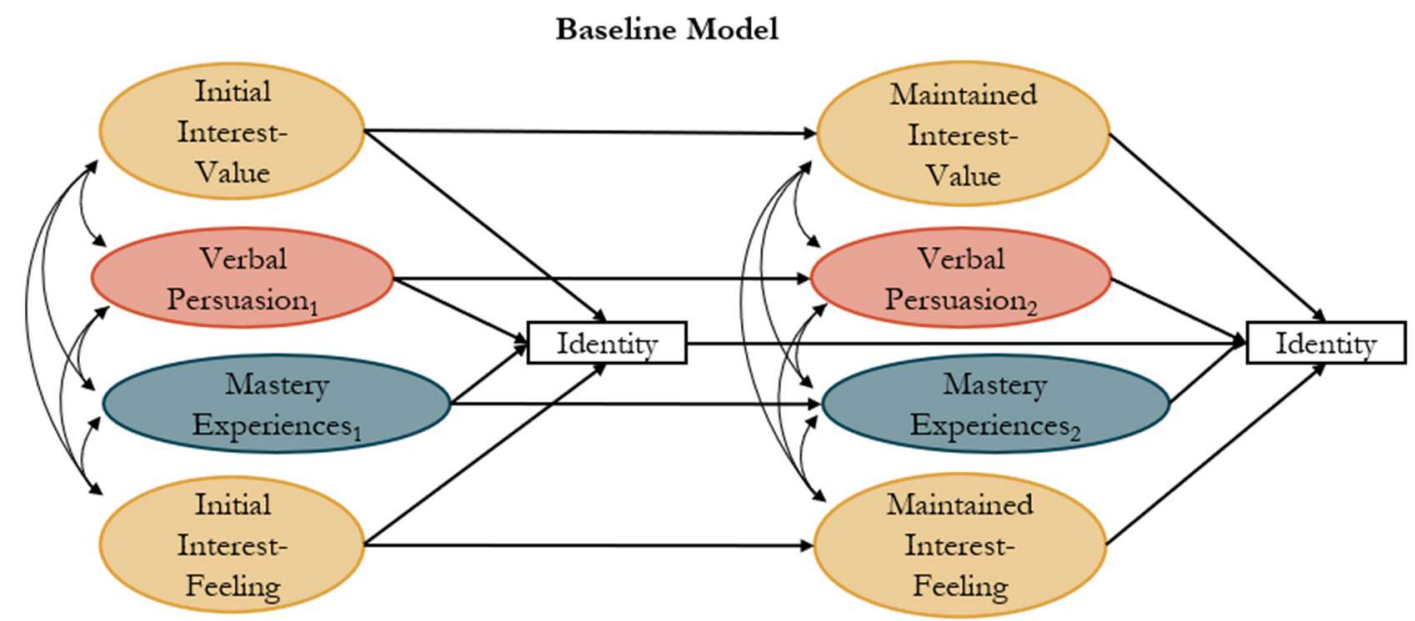

Alternative Model

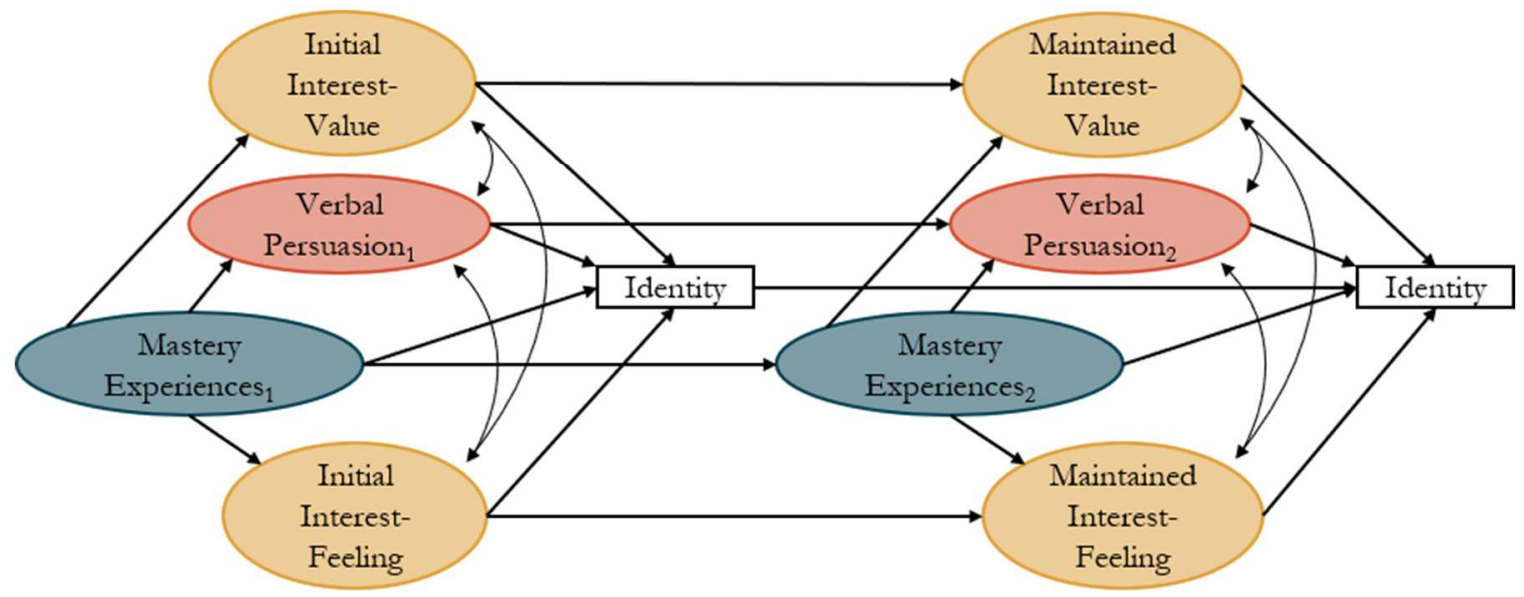

Figure 3.1. Proposed Phase Two SEM models 


\title{
Chapter 4
}

\section{Alignment of Theoretically Grounded Constructs for the Measurement of Science and Chemistry Identity}

Reprinted (adapted) with permission from Kathryn Hosbein and Jack Barbera, Alignment of Theoretically Grounded Constructs for the Measurement of Science and Chemistry Identity. Chemistry Education Research and Practice, 2020. doi:

10.1039/C9RP00193J Copyright (2020) Royal Society of Chemistry. This

manuscript cannot be reprinted or copied without permission from the publisher.

\begin{abstract}
Identity has been theorized to aid in student persistence within STEM disciplines.

In this study, science and chemistry identity were defined as being recognized as a science or chemistry person within the classroom. To generalize the effects that identity has on student persistence, a measurable construct must be defined, operationalized, and tested in multiple settings with different populations. This project addressed the first step in the process, defining the construct and grounding it in an established theoretical framework. This qualitative project utilized a previously described physics identity framework, with sub-constructs of performance/competence, recognition, and interest, as a starting point for the alignment of students' perceptions of identity to the broader theoretical frameworks of identity. Nine semi-structured interviews were conducted with students from a range of chemistry courses at Portland State University. The interviews consisted of questions pertaining to the sub-constructs of identity. Thematic analysis was used to define emerging themes within student responses. These themes were found to align with an array of affective constructs, including mastery experiences, verbal persuasion, vicarious experiences, situational interest, and mindset. These constructs will
\end{abstract}


be used to develop an identity measure for chemistry education that is grounded in the broader theoretical frameworks of identity.

\section{Introduction}

While students often select their career trajectory before entering college, (Bandura et al., 2001; Eccles, 2007) there still exists a drastic discrepancy between students entering college with a declared STEM major and graduating with a STEM degree (National Research Council, 2012; President's Council of Advisors on Science and Technology, 2012). This is especially an issue for underrepresented groups of students such as women, black, and Hispanic students (Bonous-Hammarth, 2000; Penner, 2015; Seymour et al., 1997). One of the proposed mechanisms to increase student persistence within the STEM fields is to foster science identity (Chang, Eagan, Lin, \& Hurtado, 2011; Estrada et al., 2011; Flowers \& Banda, 2016; Graham et al., 2013; President's Council of Advisors on Science and Technology, 2012). While identity has been widely proposed to increase persistence within the broad field of STEM, it has also been hypothesized to increase persistence within the more narrow discipline of chemistry (Shedlosky-Shoemaker \& Fautch, 2015).

Identity is a complex psychological construct that can be individually (Burke \& Stets, 2009) or socially (Abrams \& Hogg, 1990) constructed. An individual can have multiple identities based on characteristics such as race (Sellers et al., 1998), gender (Davidoff \& Hall, 1987; Lorber, 1994) and social status (Davidoff \& Hall, 1987). Therefore, when studying identity, it is crucial to ground identity within the specific context under study (Brickhouse et al., 2000). In 2000, James Paul Gee proposed that 
identity be used as a lens to study education and defined the construct in the following manner:

Being recognized as a certain "kind of person," in a given context, is what I mean here by "identity." In this sense of the term, all people have multiple identities connected not to their "internal states" but to their performances in society. This is not to deny that each of us has what we might call a "core identity" that holds more uniformly, for ourselves and others, across contexts (p. 99)."

Through this definition, science identity can be defined as "being recognized as a 'science person', in a science context", such as a classroom. Chemistry identity can similarly be defined as "being recognized as a "chemistry person' in a chemistry context".

Building upon Gee's definition of identity, Carlone and Johnson (2007) proposed a science identity theory that contained three sub-constructs involved in identity formation for women of color through their late college and early career paths: performance, competence, and recognition (Figure 4.1A). Performance was defined as "social performance of relevant scientific practices—e.g., ways of talking and using tools." Competence was defined as "knowledge and understanding of science content (may be less publicly visible than performance)." Recognition was defined as "recognizing oneself and getting recognized by others as a 'science person." The three constructs were hypothesized to work together such that a person would perform tasks in a science context that illustrate their competence and, in this way, the individual would be recognized by others as a credible science person. This theory accounts for the socially (as opposed to individually) constructed nature of science identity, as there are certain societal norms associated with science performance, competence, and recognition and therefore the theory assumes that one's racial, ethnic, and gender identity may overlap 
with one's science identity. While Carlone and Johnson (2007) developed a more clearly defined science identity theory through a qualitative viewpoint, it could not be generalized to larger populations because it was developed around a very specific population.

To generalize the effects that identity has on one's persistence, a measurable construct must be established and tested in multiple settings with different populations. Additionally, to support the validity of measured data, the measure needs to be operationalized specifically for its intended use. There are existing science and disciplinespecific identity measures within the education literature, however, they have been operationalized to roles other than "science person", such as "scientist" identity (Chemers et al., 2011; Estrada et al., 2011) and "science student" identity (Stets et al., 2017) or have been operationalized specifically to physics (Cass et al., 2011; Godwin \& Potvin, 2013; Hazari et al., 2010; Vincent-Ruz \& Schunn, 2018), math (Cass, et al., 2011), or science (Vincent-Ruz and Schunn, 2018). Brickhouse and colleagues (2000) have stressed the importance of studying appropriate communities of practice in which identities can form. For example, the term scientist could pose a narrow view of what it means to engage in science from a student's perspective because it implies that an individual is actively engaging in science outside of the classroom. Therefore, studying a research scientist's community of practice (e.g., using the word scientist rather than science person) when asking students questions about their science identity could be problematic. The term scientist could be irrelevant to college students depending on their experience with science outside of school, especially in the beginning of their undergraduate career when 
students may have no research experience. Measures of research scientist identity have been used to study the impacts of science support experiences such as research and mentoring that occur outside of the classroom (Chemers et al., 2011; Estrada et al., 2018; Robnett et al., 2015), but they may not be appropriate for identity changes within the classroom. One measure of science identity was designed to specifically address a single intervention within the classroom and is therefore useful for the assessment of that specific intervention (Childers \& Jones, 2017). While there are several identity measures, many of them lack the appropriate context or are not designed to specifically address chemistry identity. While a specific measure of chemistry identity has not been developed, the physics identity measure provides a starting point for its development.

\section{History of the Physics Identity Measure}

Hazari and colleagues, (2010) operationalized and modified Carlone and

Johnson's (2007) science identity theory (Figure 4.1A) and developed a theoretical framework for physics identity for the purpose of building a physics identity measure. In the physics identity framework (Hazari et al., 2010), social-cognitive career theory (SCCT) (Lent et al., 1994), which is heavily based on Bandura's social cognitive theory (SCT) (Bandura \& National Inst of Mental Health, 1986), was used to ground the overall physics identity theory. SCCT "emphasizes the means by which individuals exercise personal agency in the career development process, as well as extra-personal factors that enhance or constrain agency (Lent et al., 1994)". The proposed model (Figure 4.1B) included the constructs of performance (belief in ability to perform required physics tasks), competence (belief in ability to understand physics content), recognition by others (recognition by others as being a good physics student), and interest (desire/curiosity to 
think about and understand physics). Three distinct modifications were made to the Carlone and Johnson science identity theory to develop the physics identity theory. First, interest was not originally included in the Carlone and Johnson science identity theory because it was based on women who were already on an established path to become scientists and therefore their interest did not need support (Carlone \& Johnson, 2007). However, Carlone and Johnson did note that interest was an important factor for identity formation. The population within the Hazari and colleagues (2010) study included students who were non-physics majors or earlier in their physics academic career, where interest may not be stable and therefore would be valuable to measure. Based on this, and evidence that interest has a large impact on career choice and therefore who or what a student wants to be (Lent et al., 1994), Hazari and colleagues (2010) decided to include the construct of interest within their physics identity framework. Second, performance and competence were re-defined to reflect confidence in ability (similar to self-efficacy) rather than purely ability. The third modification between the theories was the conceptualization of recognition. Within the Carlone and Johnson identity theory, selfrecognition was combined with recognition by others, whereas Hazari and colleagues (2010) viewed self-recognition as a core feature that could be influenced by whether an individual is recognized by others. Therefore, self-recognition was theorized as a separate holistic identity variable consisting of a single item that asked "Do you see yourself as a physics person?" (Potvin \& Hazari, 2013; Shanahan, 2009). Items based on these constructs were tested and, after exploratory factor analysis (EFA), a revised version of the framework that consisted of performance/competence as a single construct, 
recognition by others, an identity variable (i.e., self-recognition) and interest (Figure

4.1C). The physics identity measure, containing items aligned to the constructs of

performance/competence, recognition, and interest along with the identity variable, has

been modified for use in other disciplines and items within the measure have gone

through multiple iterations and psychometric testing (Cass et al., 2011; Cheng et al.,

2018; Godwin, Potvin, \& Hazari, 2013; Verdín et al., 2018).

SCIENCE IDENTITY

(Carlone and Johnson 2007)
PHYSICS IDENTITY

(Hazari et. al., 2010)
B)

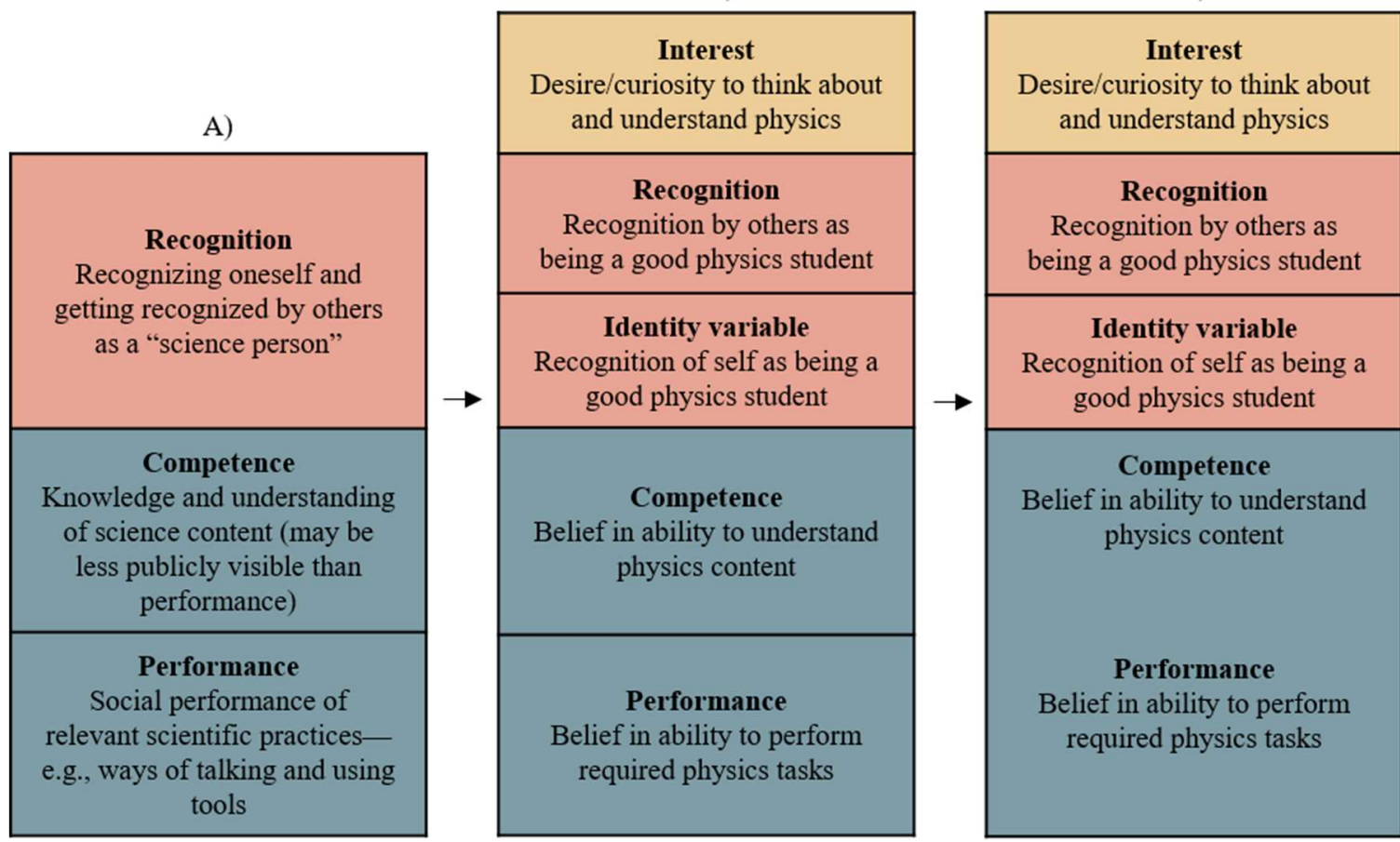

Figure 4.1. The theoretical frameworks for A) science identity, B) physics identity, and C) physics identity after modification.

\section{Initial physics identity items}

While Hazari and colleagues, (2010) described the grounding of their physics

identity theoretical framework, the process for development of the sub-construct items is somewhat unclear. The first physics identity items (associated with the constructs described in Figure 4.1B) were part of a large national survey from the Persistence 
Research in Science and Engineering (PRiSE) Project. The PRiSE survey set out to explore factors from high school that influence persistence of females within STEM and was developed using three methods: a literature review of constructs that affect persistence, open-ended responses from 259 secondary science teachers and 153 scientists on what they believe influences persistence in college, and extraction of items from a previously used national survey (Factors Influencing College Success-FICSS). In total, the PRiSE survey was comprised of 50 items across 6 sections, requiring more than 250 individual responses from students. The original physics identity survey was compiled from a range of PRiSE items. However, few details were provided regarding why the items were chosen or how they were assigned to each sub-construct of identity through the theoretical framework of SCCT.

\section{Performance/Competence}

Performance was measured using five PRiSE items about middle school and high school grades along with SAT scores. It is of note here that the construct of performance was measured using student ability in the form of past grades, whereas the definition of performance within the physics identity framework described performance as belief in ability. Competence was measured using two PRiSE items about students' perceived confidence in middle school math and science. Within SCCT, the construct of performance is theorized to influence one's self-efficacy and therefore career goals and choices while the construct of competence is not specifically mentioned.

\section{Interest}

Nineteen PRiSE items were used to measure the construct of interest. These items reflected interest in specific tasks associated with three domains: physics interest (e.g., 
mechanics and electromagnetism), science interest (e.g., understanding natural phenomena and using mathematics), and science activity (e.g., participation in science groups/clubs/camps and science/math competitions). Within SCCT, interest is defined as “...likes, dislikes, and indifferences regarding career-relevant activities and occupations (Lent et al., 1994, p. 88)". The interest items on the PRiSE survey were not explicitly tied to career-relevant activities or occupations.

\section{Recognition}

Items measuring recognition on the PRiSE survey included items where students rated whether certain groups (i.e., their science teacher or their parents/relatives/friends) saw them as a physics person. These two items of recognition were retained for the physics survey, however, the definition of recognition within the physics identity framework pertains to whether a student is recognized as a good "physics student". These wording differences could conflict based on students" definitions of "physics person". Recognition is not specifically mentioned in SCCT but does align well with the verbal persuasion source of self-efficacy defined within SCT (Bandura \& National Inst of Mental Health, 1986, pp. 405-406), although this alignment is not mentioned by Hazari and colleagues (2010).

\section{Further modifications}

Subsequent publications have utilized the Hazari and colleagues (2010) framework (Figure 4.1C) with item modifications (Cheng et al., 2018; Godwin, Potvin, Hazari, et al., 2013; Godwin et al., 2016). However, little to no explanation of the decisions to add or delete items or change item wordings has been described. For example, more recent versions contain broadly worded interest items such as "I am 
interested in learning more about the subject [physics]" (Godwin, Potvin, \& Hazari, 2013) and "I enjoy learning about physics" (Cheng et al., 2018), as compared to the original items that described interest with regard to specific content within physics. While these items were developed based on performance, competence, interest, and recognition proposed by Hazari and colleagues (2010), the process of development to ensure that items reflected each sub-construct remains unclear. Due to the lack of clear theoretical connections to the sub-constructs of identity and the items developed to measure them, it is worth exploring the sub-constructs and their connection to established affective constructs within the literature.

\section{Purpose and Rationale for the Study}

While psychometrics, such as EFA, are crucial to validity, it is equally as important to show the development of measures based on an established theory (Furr \& Bacharach, 2008, pp. 172-173; Kline, 2016, p. 94). While items designed to reflect performance/competence, interest, and recognition have been used in prior studies of student identity (Cheng et al., 2018; Cribbs et al., 2015; Godwin, Potvin, Hazari, et al., 2013; Verdín et al., 2018) and shown evidence of valid data through psychometrics, there has been little evidence to support the theoretical backing of the items used to measure these sub-constructs of the physics identity framework. Providing evidence for the alignment between items and the construct being measured is crucial to content validity (Furr \& Bacharach, 2008, pp. 172-173). Therefore, this study used semi-structured interviews to provide further evidence of content validity through two related aims. The first aim of this study was to explore the constructs of performance/competence, recognition, and interest, within the physics identity framework, through student 
responses to previously used items. The second aim was to build upon the physics identity framework by exploring the connections between the sub-constructs of identity and theoretically grounded affective constructs. Rooting the sub-constructs of identity within a defined theoretical framework is the primary step in our development of measures of identity for use within chemistry education.

The aims of this project will be carried out through addressing the following research questions:

1) What themes arise when students are asked questions reflecting performance/competence, recognition, and interest, pertaining to science or chemistry, as described by the physics identity framework?

2) To what extent do reported themes align with proposed and other affective constructs?

\section{Methods}

\section{Participants}

After obtaining institutional review board (IRB) approval for the study, the sample consisted of students in undergraduate chemistry courses at Portland State University (PSU). To sample a range of student levels (e.g., by major and year in degree), the selected courses included an off-sequence general chemistry course, two sections of an organic chemistry course, and a biochemistry course for non-biochemistry majors. Student enrollment for the four courses were 233, 162, 131, and 235, respectively. A final question on a two-question recruitment survey asked students if they were willing to participate in a follow-up interview. Students who responded positively to the question were recruited and randomly selected for interviews. To capture a range of responses, 
quota sampling was used (Tourangeau \& Yan, 2012), where an equal number of participants per specified responses were selected. Responses to the two items, "I see myself as a science person" and "I see myself as a chemistry person" from the related survey, were used to select participants. Students who agreed to participate in an interview were separated into three groups: 1) those who agreed with both statements (by selecting "agree" or "strongly agree" on the Likert scale for both items), 2) those who did not agree with both statements (by selecting "neutral", "disagree", or "strongly disagree" for both items), and 3) those who had selected "neutral", "disagree", or "strongly disagree" for the chemistry identity item and "agree" or "strongly agree" for the science identity item. Of note, there were no students who volunteered for an interview who had selected "neutral", "disagree", or "strongly disagree" for the science identity item and "agree" or "strongly agree" for the chemistry identity item. One student from each of the three groups was then randomly selected for an interview. This was repeated until there was one student per group per course type, for a total of nine interviews.

\section{Data Collection}

A semi-structured interview format was used to investigate student responses to questions designed to target performance/competence, recognition, and interest, as outlined in the physics identity framework. First, participants were asked two open-ended questions: What makes someone a science person? and What makes someone a chemistry person? Next, items from an instrument designed from the physics identity theory (Godwin, Potvin, \& Hazari, 2013) were used. Items describing each sub-construct of physics identity have gone through multiple iterations (Cheng et al., 2018; Cribbs et al., 2015; Godwin, Potvin, Hazari, et al., 2013; Verdín et al., 2018). Therefore, only the items 
that have been the most consistently used within the literature were utilized in this study. These items were modified for the purpose of being posed as open-ended questions to students. For example, the item "I see myself as a science person" was modified to "Do you see yourself as a science person?" After asking the science worded version of the question and any relevant follow-up questions, the chemistry worded version of the question was asked, i.e., "Do you see yourself as a chemistry person?" These open-ended questions formed the semi-structured interview protocol (included in the Appendix).

\section{Analysis}

Interviews were transcribed using a professional transcription service. The transcribed interviews were then analyzed using a thematic analysis framework (Braun et al., 2019; Gibson \& Brown, 2009, pp. 127-144; Saldaña, 2015, pp. 287-294) with the software MAXQDA (Version 18.2.0). Thematic analysis is a method used to describe themes (patterns of meaning) within the data. There are many ways to utilize thematic analysis, but regardless of how it is utilized, it is crucial for researchers to report the way the method was used so that the assumptions of the method are clear (Braun \& Clarke, 2006). The thematic analysis was performed through an essentialist lens (Braun \& Clarke, 2006), which assumes the responses of participants directly reflect their experiences. To obtain a holistic picture of participant responses when asked about performance/competence, recognition, and interest, the thematic analysis was used as an inductive method of analysis as compared to a deductive analysis method, where a priori codes are developed. The process of data analysis was carried out both independently and collaboratively by the first author and an undergraduate researcher trained in chemistry education. The reflexive thematic analysis used in this study followed five steps as 
described by Braun and Clarke (2019): 1) familiarization, 2) generating codes, 3) constructing themes, 4) revising themes, and 5) defining themes. First, two of the nine transcripts were read independently and multiple times to gain familiarity with the data. During these reads, each researcher recorded notes on patterns within the two transcripts. The second step contained multiple sub-steps. The two researchers came together to record all identified patterns from their notes and combined similar patterns when necessary. A codebook with codes and definitions was then created based on the final list of patterns. This codebook was then used by the researchers to independently code two additional transcripts and notes of new codes were recorded. Once again, the researchers came together to refine codes and edit the codebook. This process was repeated one more time with two additional transcripts until a final codebook was established. Reflexive thematic analysis does not use strict measures of inter-rater reliability. Coding is considered an iterative process where codes are continually developed throughout analysis (Braun et al., 2019). In this case, the codebook was considered complete when the two researchers reached a consensus that there were no more unique codes. The final codebook was used by the researchers to independently code two new transcripts to confirm that no new codes were discovered. Once the final codebook was confirmed, the last transcript was coded and all transcripts were re-coded to consensus. To explore the prevalence of codes within specific portions of the interviews, transcripts were divided into four sections according to Table 4.1. 
Table 4.1. Division of interviews into sections by grouped items. The word [science] was replaced with chemistry for the chemistry-worded version of each question.

\begin{tabular}{ll}
\hline Section & Questions included \\
\hline Attributes and Self-Recognition & What do you think makes someone a [science] person? \\
& Do you see yourself as a [science] person? \\
\hline Recognition by Others & Do your friends see you as a [science] person? \\
& Do your peers see you as a [science] person? \\
& Do people who are important to you see you as a [science] \\
& person? \\
& Have you had specific experiences that you can recall where \\
& you feel like you've been recognized as a [science] person? \\
\hline Performance/Competence & How confident are you that you can understand [science] in \\
& class? \\
& How confident are you that you can understand [science] \\
& outside of class? \\
& Do you do well on exams in [science]? \\
& Do others as you for help in [science]? \\
& Have you overcome any setbacks in [science]? \\
\hline Interest & Are you interested in learning more about [science]? \\
& Do you enjoy learning [science]? \\
& Do you find fulfillment in doing [science]? \\
\hline
\end{tabular}

After all transcripts were coded, the final three steps of analysis consisted of evaluating the generated codes for themes, revising, and defining codes. Codes were rearranged into groups multiple times until the first author decided on a final set of groupings that resulted in themes. The themes were then defined by the first author, reviewed by the second author, and subsequently discussed with secondary researchers. The final themes were used to explore connections of the sub-constructs of performance/competence, recognition, and interest with established affective constructs.

\section{Results and Discussion}

Nine students participated in semi-structured interviews that included questions about performance/competence, recognition, and interest and overall science and chemistry identity. Table 4.2 shows demographic information for each participant and the responses to the items used for interview selection. Pseudonyms were used to protect 
each participant's identity. While the participating sample is almost entirely female and Caucasian, the participant selection for interviews was randomized among those who volunteered to take place in an interview and met our quota sampling criteria. In total, seventeen females and six males were randomly recruited from the pool of volunteers. Of those recruited, nine females and five males either declined or did not respond to scheduling an interview.

Table 4.2. Pseudonyms and demographics for interview participants.

\begin{tabular}{|c|c|c|c|c|c|c|}
\hline Name & Course* & $\begin{array}{l}\text { Gender } \\
\text { Identity }\end{array}$ & Race** & $\begin{array}{l}\text { University } \\
\text { Status*** }\end{array}$ & $\begin{array}{c}\text { I see myself as } \\
\text { a science } \\
\text { person }\end{array}$ & $\begin{array}{c}\text { I see myself as } \\
\text { a chemistry } \\
\text { person }\end{array}$ \\
\hline Max & $\mathrm{GC}$ & Female & Caucasian & Undergrad & Strongly agree & Strongly agree \\
\hline Nancy & $\mathrm{OC}$ & Female & Polynesian & Undergrad & Agree & Agree \\
\hline Karen & $\mathrm{BC}$ & Female & Caucasian & Transfer/Undergrad & Strongly agree & Agree \\
\hline Barb & $\mathrm{GC}$ & Female & Caucasian & Undergrad & Agree & Disagree \\
\hline Joyce & $\mathrm{OC}$ & Female & $\begin{array}{c}\text { Caucasian } \\
\text { /Asian }\end{array}$ & Undergrad & Agree & Disagree \\
\hline Steve & $\mathrm{BC}$ & Male & Caucasian & Undergrad & Strongly agree & $\begin{array}{l}\text { Strongly } \\
\text { disagree }\end{array}$ \\
\hline Elle & GC & Female & Caucasian & Undergrad & $\begin{array}{l}\text { Strongly } \\
\text { disagree }\end{array}$ & $\begin{array}{l}\text { Strongly } \\
\text { disagree }\end{array}$ \\
\hline Erica & $\mathrm{OC}$ & Female & Slavic & Post-bac & Disagree & Disagree \\
\hline Robin & $\mathrm{BC}$ & Female & Caucasian & Post-bac & Disagree & Disagree \\
\hline \multicolumn{7}{|c|}{$\begin{array}{l}* \mathrm{GC}=\text { General Chemistry, } \mathrm{OC}=\text { Organic Chemistry, } \mathrm{BC}=\text { Biochemistry, } * * \text { race was provided through an } \\
\text { open ended, self-report format, } * * * \text { undergrad refers to students on a traditional college path, transfer refers } \\
\text { to students who transferred to PSU from a } 2 \text { or } 4 \text { year college or university, and post-bac refers to students } \\
\text { who already had a bachelor's degree and were returning to school for a secondary degree or pre-requisites } \\
\text { for a graduate level program. }\end{array}$} \\
\hline
\end{tabular}

\section{Identified Codes}

Twelve codes were determined throughout the nine semi-structured interviews.

The codebook was created using six of the nine transcripts. When using the codebook to analyze the final three transcripts, no novel codes were discovered, which provided evidence of data saturation (Guest, Bunce, \& Johnson, 2006). Codes, their definitions, and examples are shown in Table 4.3. 
Table 4.3. Codes, their definitions, and examples of codes within the text.

\begin{tabular}{|c|c|c|}
\hline$\overline{\text { Code }}$ & Definition & Example of Segment from Text \\
\hline Feelings & $\begin{array}{l}\text { Positive or negative feelings associated } \\
\text { with science or chemistry (e.g., enjoy, } \\
\text { interesting, fun, boring, overwhelming). }\end{array}$ & "...I love biology..." \\
\hline $\begin{array}{l}\text { Real-world } \\
\text { application of } \\
\text { science or } \\
\text { chemistry }\end{array}$ & $\begin{array}{l}\text { Science or chemistry in conjunction with } \\
\text { its applications to the real-world/everyday } \\
\text { life or to career. Science or chemistry with } \\
\text { technical applications (i.e., using hands to } \\
\text { perform science). Science or chemistry as a } \\
\text { form of altruism. Science or chemistry as a } \\
\text { way to create new technologies } \\
\text { (innovations) and solve problems. }\end{array}$ & $\begin{array}{l}\text { "I do enjoy learning some of the concepts } \\
\text { and some of the real-world... aspect of it." }\end{array}$ \\
\hline Goals & $\begin{array}{l}\text { Science or chemistry is used as a means to } \\
\text { an end. }\end{array}$ & $\begin{array}{l}\text { "I wanna go to medical school so that's stuff } \\
\text { I would like to learn more about." }\end{array}$ \\
\hline $\begin{array}{l}\text { Science or } \\
\text { chemistry as an } \\
\text { epistemology }\end{array}$ & $\begin{array}{l}\text { Science or chemistry used as a way of } \\
\text { obtaining knowledge about the world. }\end{array}$ & $\begin{array}{l}\text { "It's the way that the whole world is made } \\
\text { up." }\end{array}$ \\
\hline $\begin{array}{l}\text { Knowledge of } \\
\text { science or } \\
\text { chemistry }\end{array}$ & $\begin{array}{l}\text { Possessing some foundational knowledge } \\
\text { of science or chemistry. Understanding of } \\
\text { science or chemistry concepts. }\end{array}$ & $\begin{array}{l}\text { "I think they have to at least have some sort } \\
\text { of knowledge of science...to make them a } \\
\text { science person." }\end{array}$ \\
\hline $\begin{array}{l}\text { Performance in } \\
\text { school }\end{array}$ & $\begin{array}{l}\text { Discussing performance in the classroom } \\
\text { and situations involving performance (e.g., } \\
\text { grades or being stressed about courses). }\end{array}$ & $\begin{array}{l}\text { "I was not expecting to do very well in the } \\
\text { tests but I ended up getting a B on the } \\
\text { final, and I was really surprised." }\end{array}$ \\
\hline $\begin{array}{l}\text { Encouragement/ } \\
\text { Discouragement }\end{array}$ & $\begin{array}{l}\text { When participant is encouraged/affirmed } \\
\text { OR discouraged by people in their life } \\
\text { including family, teachers, friends, peers. } \\
\text { Also includes feeling encouraged by } \\
\text { receiving awards. }\end{array}$ & $\begin{array}{l}\text { "...my parents are always encouraging and } \\
\text { supportive of me in science areas and ever } \\
\text { since I was young." }\end{array}$ \\
\hline $\begin{array}{l}\text { Science or } \\
\text { chemistry in } \\
\text { conversation }\end{array}$ & $\begin{array}{l}\text { Science or chemistry is discussed in } \\
\text { various ways with others such as debating, } \\
\text { using scientific jargon, or discussing } \\
\text { science or chemistry in general. }\end{array}$ & $\begin{array}{l}\text { "Yes. I mean I'm always one to debate or } \\
\text { just discuss--go on hypothetical voyages of } \\
\text { things about science." }\end{array}$ \\
\hline $\begin{array}{l}\text { Explaining } \\
\text { science or } \\
\text { chemistry to } \\
\text { others }\end{array}$ & $\begin{array}{l}\text { The participant's competency in science or } \\
\text { chemistry is expressed to others through } \\
\text { explaining science or chemistry concepts. }\end{array}$ & $\begin{array}{l}\text { "I'm an anatomy person, so they'll ask me } \\
\text { certain things about the body or whatever. } \\
\text { And so when it comes to subjects of science } \\
\text { that I know, then they'll ask me questions } \\
\text { about that. And then they'll know that I } \\
\text { know the answers and stuff like that." }\end{array}$ \\
\hline
\end{tabular}


Table 4.3 cont.

\begin{tabular}{|c|c|c|}
\hline Code & Definition & Example of Segment from Text \\
\hline $\begin{array}{l}\text { Comparison to } \\
\text { others }\end{array}$ & $\begin{array}{l}\text { Referencing their place in science or } \\
\text { chemistry based on others. }\end{array}$ & $\begin{array}{l}\text { "My boyfriend really enjoys chemistry and } \\
\text { biology, and I'm like, 'how?' I don't } \\
\text { understand." }\end{array}$ \\
\hline $\begin{array}{l}\text { Intuition for } \\
\text { understanding } \\
\text { science or } \\
\text { chemistry }\end{array}$ & $\begin{array}{l}\text { Science or chemistry comes easily or } \\
\text { naturally. Science or chemistry takes a } \\
\text { certain type of thinking (e.g., strong } \\
\text { visual/conceptual perception of abstract } \\
\text { concepts). }\end{array}$ & "... it doesn’t come naturally." \\
\hline Determination & $\begin{array}{l}\text { Persisting through challenges in science or } \\
\text { chemistry. }\end{array}$ & $\begin{array}{l}\text { "And I think at first it was pretty } \\
\text { challenging for me and just trying to think } \\
\text { about all the new kind of concepts but I was } \\
\text { able to ask for help and work through it } \\
\text { and I was able to do it." }\end{array}$ \\
\hline
\end{tabular}

It is important to note that codes were assigned to any portion of the text that contained the description of the code throughout the entire interview, not simply in the response connected to a subset of questions. The prevalence of each code was determined for each of the four sections in the interviews as described in Table 4.1: Attributes and SelfRecognition, Recognition by Others, Performance/Competence, and Interest. In addition to the four sections, the responses to science and chemistry worded questions were combined when analyzed for the prevalence of codes. For example, the responses from "Do your friends recognize you as a science person" and "Do your friends recognize you as a chemistry person?" If a code was present within both responses, it was only counted one time. This was done because students sometimes conflated science and chemistry when asked specifically about science. Therefore, chemistry follow up questions were not always asked. The number of participants that mentioned each code within each section is shown in Table 4.4. Portions of text within responses could be coded with more than one code, sometimes resulting in multiple codes per response per participant. The constructs 
of performance/competence, recognition, and interest have been theorized to correlate, therefore it was not a concern that single codes appeared in multiple sections of the interview. Therefore, to clarify how many unique interviews contained the code, an "overall" column was added to Table 4.4. For example, in the first row of the table, the code "Goals" was applied to the discussions of six interview participants. For these participants, the "Goals" code was differentially applied within each of the four sections of the interview. It can be seen that while each of these participants discussed "Goals" during the Interest section of their interview, fewer discussed this aspect within the other sections, with only one discussing "Goals" within their Performance/Competence section.

Table 4.4. The number of interviews and sections that contained each individual code.

\begin{tabular}{|c|c|c|c|c|c|}
\hline \multirow[b]{2}{*}{ Codes } & \multirow[b]{2}{*}{$\begin{array}{c}\text { Number } \\
\text { of } \\
\text { Interviews }\end{array}$} & \multicolumn{4}{|c|}{ Interview Sections } \\
\hline & & $\begin{array}{c}\text { Attributes } \\
\text { and Self- } \\
\text { Recognition }\end{array}$ & $\begin{array}{l}\text { Recognition } \\
\text { by Others }\end{array}$ & $\begin{array}{l}\text { Performance/ } \\
\text { Competence }\end{array}$ & Interest \\
\hline Goals & 6 & 4 & 5 & 1 & 6 \\
\hline Feelings & 9 & 9 & 7 & $\mathrm{~N} / \mathrm{A}^{*}$ & 8 \\
\hline Real-world application & 9 & 7 & 4 & 6 & 9 \\
\hline Science as an epistemology & 7 & 7 & 2 & $\mathrm{~N} / \mathrm{A}$ & 5 \\
\hline $\begin{array}{l}\text { Knowledge of science or } \\
\text { chemistry }\end{array}$ & 6 & 4 & 4 & 5 & 4 \\
\hline Performance in school & 9 & 6 & 5 & 9 & 1 \\
\hline Encouragement & 6 & 1 & 5 & 1 & N/A \\
\hline $\begin{array}{l}\text { Science or chemistry in } \\
\text { conversation }\end{array}$ & 8 & 1 & 8 & 2 & 1 \\
\hline $\begin{array}{l}\text { Trusted as a science or } \\
\text { chemistry source }\end{array}$ & 9 & $\mathrm{~N} / \mathrm{A}$ & 6 & 6 & N/A \\
\hline $\begin{array}{l}\text { Comparison of self with } \\
\text { others }\end{array}$ & 8 & 2 & 7 & 3 & N/A \\
\hline Intuition for understanding & 9 & 8 & 1 & 2 & N/A \\
\hline Determination & 6 & 4 & 4 & 5 & 4 \\
\hline
\end{tabular}

*N/A indicates that the code did not appear in this section within any interviews.

\section{Emergent Themes}

Codes were combined to create overarching themes that were discussed when students were asked about performance/competence, recognition, interest, and overall science and chemistry identity. Four themes were determined from the twelve codes 
present within the semi-structured interviews. Themes, the codes contained within each theme, and an overall description of the themes are contained in Table 4.5. Each theme and how it was used within the interviews is described below.

Table 4.5. Themes, codes contained within each theme, and a description of each theme that are present throughout the interviews.

\begin{tabular}{|c|c|c|}
\hline Theme & Codes & Description \\
\hline $\begin{array}{l}\text { Interest in science or } \\
\text { chemistry is based on } \\
\text { feelings or values and } \\
\text { occurs in stages. }\end{array}$ & $\begin{array}{l}\text { Feelings, real-world } \\
\text { application, goals, science as } \\
\text { an epistemology }\end{array}$ & $\begin{array}{l}\text { Interest in science or chemistry was } \\
\text { described using feelings or interest tied to } \\
\text { values such as the real-world applications of } \\
\text { science or chemistry, goals that depend on } \\
\text { science or chemistry, or science and } \\
\text { chemistry as a tool to solve problems. } \\
\text { Amount of interest in science or chemistry } \\
\text { varied between students. }\end{array}$ \\
\hline $\begin{array}{l}\text { Educational experiences } \\
\text { contribute to student } \\
\text { science or chemistry } \\
\text { identity. }\end{array}$ & $\begin{array}{l}\text { Knowledge of science or } \\
\text { chemistry, performance in } \\
\text { school }\end{array}$ & $\begin{array}{l}\text { Participation in science or chemistry was } \\
\text { described using examples from educational } \\
\text { experiences. }\end{array}$ \\
\hline $\begin{array}{l}\text { Students gain } \\
\text { information about } \\
\text { identity through } \\
\text { interactions with others. }\end{array}$ & $\begin{array}{l}\text { Encouragement, science or } \\
\text { chemistry in conversation, } \\
\text { trusted as a science or } \\
\text { chemistry source, comparison } \\
\text { of self with others }\end{array}$ & $\begin{array}{l}\text { The relation of a participant to science or } \\
\text { chemistry was discussed in terms of gaining } \\
\text { information about oneself through } \\
\text { interacting with people in their lives. }\end{array}$ \\
\hline $\begin{array}{l}\text { Participation in science } \\
\text { or chemistry takes a } \\
\text { certain type of person. }\end{array}$ & $\begin{array}{l}\text { Intuition for science or } \\
\text { chemistry, determination }\end{array}$ & $\begin{array}{l}\text { Certain types of people are more suited } \\
\text { toward participating in science or chemistry } \\
\text { based on certain traits. }\end{array}$ \\
\hline
\end{tabular}

Theme 1: Interest in science or chemistry is based on feelings or values and occurs in stages. Interest is based on feelings and values.

Students frequently described interest in science or chemistry based on their feelings or values throughout the interviews. Representative student quotes can be found within Table 4.6. All students referenced feelings toward science throughout the Attributes and Self-Recognition section of the interviews. For example, Robin mentioned excitement when describing the attributes of a science person. Erica used words such as exciting, entertaining, and fun to describe how her professor sees chemistry and was the 
reason she saw him as a chemistry person as compared to herself. Within the Recognition section, when asked if others see them as a science or chemistry person, some students, such as Max and Joyce, thought others knew about their interest (or disinterest) in the subject and was a form of being recognized (or not recognized) as a science or chemistry person. Additionally, these feeling-related words were used by students to describe reasons they did or did not recognize themselves as science or chemistry people. Examples come from Steve and Elle within the Attributes and Self-Recognition section, as they use words such as psyched, love, and enjoy when describing their feelings toward chemistry.

In addition to feelings, students frequently mentioned real-world applications, goals, or using science as a tool to obtain knowledge when responding to questions. These types of references are reflective of their interest related to values, i.e., they are interested in science or chemistry because it is valuable, or important to them (Schiefele, 1991). For example, Robin and Barb described their career goals within the medical field as drivers of their interest in science, and Erica described real-world application in relation to her interest in chemistry. The discussion of real-world applications of science provided a link between the value of science pertaining to Barb's interest and how this affected her confidence. Within the Attributes and Self-Recognition portion of the interview, Barb described being interested in science because of the real-world application of the subject and later, in the Performance/Competence section, described how real-world applications aided in her confidence of biology. 
Table 4.6. Student quotes pertaining to feeling- and value-related interest.

\begin{tabular}{|c|c|c|}
\hline Interest Component & Student & Quote \\
\hline \multirow[t]{6}{*}{ Feeling } & Robin & $\begin{array}{l}\text { "I think if people are really excited about science that would make } \\
\text { them kind of a science person." }\end{array}$ \\
\hline & Erica & $\begin{array}{l}\text { "Yeah, I don't see myself as a chemistry person. I had a good } \\
\text { professor who I can totally...he's a chemistry person. He gets really } \\
\text { excited when he talks about it, and he finds it entertaining and fun." }\end{array}$ \\
\hline & Max & "I think that they could tell that I like [science]..." \\
\hline & Joyce & $\begin{array}{l}\text { "I complain a lot. Yeah, I don't think they would [see me as a } \\
\text { chemistry person]." }\end{array}$ \\
\hline & Steve & $\begin{array}{l}\text { "I come home every day after class just psyched. The other day in } \\
\text { biochem we were learning about Warberg shifts in cancer cells and } \\
\text { I'm just wide eyed and innocent. Oh, I love it." }\end{array}$ \\
\hline & Elle & "I don't enjoy [chemistry]." \\
\hline \multirow[t]{5}{*}{ Value } & Robin & $\begin{array}{l}\text { "For me personally I'm hoping to think about sort of medical stuff or } \\
\text { the medicine or nursing. I feel like that's really applicable to helping } \\
\text { people so that feels good and I mean I think science is very } \\
\text { important." }\end{array}$ \\
\hline & Barb & $\begin{array}{l}\text { "Doing science for me is ... Well, learning science and doing science } \\
\text { for me is for so that I can go out into the health field. And so I think } \\
\text { that that's fulfilling for me to go help people in the future." }\end{array}$ \\
\hline & Erica & $\begin{array}{l}\text { "Chemistry gives me an insight on enzymes, and how I didn't know } \\
\text { about inflammation, and why temperature goes up, and how ... It's } \\
\text { just mind blowing how our body runs on electricity. These little } \\
\text { things, I think it's truly fascinating, and I think everyone should } \\
\text { know that." }\end{array}$ \\
\hline & Barb & $\begin{array}{l}\text { "I think science is fascinating. I think just the subject of science and } \\
\text { how it relates, like I said at the beginning, how it relates to the real- } \\
\text { world...And that's something that you can apply in your every day } \\
\text { life." }\end{array}$ \\
\hline & Barb & $\begin{array}{l}\text { "...I had a biology [course] last term, so we went out and were } \\
\text { learning a lot about plants outside. And so I was able to kind of go } \\
\text { out there. Oh, I know that's this plant, or that's this plant. And so } \\
\text { that's kind of brings boost in my confidence." }\end{array}$ \\
\hline
\end{tabular}

Interest occurs in stages

In addition to discussing feelings and values when describing interest in science

or chemistry, students seemed to be in varying stages of interest (Hidi \& Renninger,

2006). Representative student quotes are available in Table 4.7. For example, Barb

described that she did not always enjoy chemistry, which suggested her interest was in 
the early stages of formation. Similarly, Steve explained his growing interest in chemistry after realizing the practical applications. After this recognition, Steve was able to engage more with the material in his biochemistry course. Nancy and Max described situations where their interest is heightened after being in the classroom, which suggested their interest was continuing to grow based on classroom material. The Table 7 examples from Barb, Steve, Nancy, and Max, suggested that student interest is heightened after learning something in the classroom and in some cases, they are re-engaging with the material themselves, such as discussing it with others.

While there were examples of students describing their growing interest, some students described having more of a solidified interest pertaining to science or chemistry. For example, when Robin was asked what makes her interested in science, she suggested that science has been integrated into the way she thinks and therefore she re-engages with science often. This supported a more concrete and stable form of interest. While the responses by students were not clear enough to confidently say what stages of interest development students were in, there was evidence that students experienced varying amounts of interest in science and chemistry. 
Table 4.7. Student quotes pertaining to varying stages of interest.

\begin{tabular}{|c|c|c|}
\hline Interest Formation Stage & Student & Quote \\
\hline \multirow[t]{2}{*}{ Early } & Barb & $\begin{array}{l}\text { "Sometimes, I do [enjoy chemistry]. I enjoy lab. I enjoy } \\
\text { learning some of that stuff, and I enjoy learning ... I do } \\
\text { enjoy learning some of the concepts and some of the } \\
\text { real-world, like I said, the real-world aspect of it." }\end{array}$ \\
\hline & Steve & $\begin{array}{l}\text { "And then all of a sudden you're like, "Oh my gosh! } \\
\text { This is cool!" Especially with biochem. ...I didn't realize } \\
\text { the practical application of chemistry. And now you } \\
\text { have whole new world of appreciation for it." }\end{array}$ \\
\hline \multirow[t]{2}{*}{ Mid } & Nancy & $\begin{array}{l}\text { "That I can learn something and then I can go } \\
\text { out... and relate it and tell people my nerdy answers." }\end{array}$ \\
\hline & $\operatorname{Max}$ & $\begin{array}{l}\text { "Like specifically with psychology or biology and you } \\
\text { learn something and then you're like so that's why that } \\
\text { happens." }\end{array}$ \\
\hline Late & Robin & $\begin{array}{l}\text { "I mean, it's a way to understand the world and...I } \\
\text { think when I can apply it to sort of real life, it's really, } \\
\text { really fascinating. And I love to kind of know about } \\
\text { how things work and I think it's also a way to help } \\
\text { people and so yeah." }\end{array}$ \\
\hline
\end{tabular}

\section{Theoretical Frameworks Surrounding Theme 1}

Students were interested in science based on their feelings and values. In addition

to feelings and values, students described varying degrees of interest. As outlined by the

physics identity framework, interest has been proposed as a construct that is a part of

domain-specific identity formation. However, the description was not specifically aligned

within a theory of interest. Based on the responses of students when discussing interest,

the construct of interest as described by the physics identity framework aligned well with

the theories that describe feeling- and value-related interest (Schiefele, 1991) and the

four-phases of interest (Renninger \& Hidi, 2011).

Feeling- and Value-related Interest

Schiefele (1991) has proposed that there are two components to interest: value-

related and feeling-related. Value-related interest refers to the significance an individual

has to a particular subject, whereas feeling-related interest refers to the positive feeling 
one relates to a particular subject. Schiefele (1991) states, "Although these two components correlate highly with one another, it seems justified to distinguish between them (p. 303)." Within the Interest section of the interviews, students discussed the construct of interest through both feelings and values. These responses directly aligned with feeling- and value-related components of interest. Words describing feelings such as fascinating, cool, and interesting were used when students were asked to describe their interest in science or chemistry. These feeling-centered words were commonly followed up with statements describing students' values such as the application of science or chemistry to the real-world and the goals students will achieve through science or chemistry.

\section{The Four-Phases of Interest}

Although the term "interest" is often used by researchers, it is not always well defined or specifically placed within a theoretical framework (Renninger \& Hidi, 2011; Schiefele, 1991). Interest can be conceptualized in many ways such as development (Hidi \& Renninger, 2006; Krapp, 2007), emotion (Ainley, 2007; Silvia, 2005), and environment (Sansone, Fraughton, Zachary, Butner, \& Heiner, 2011). While there are multiple conceptualizations of overall interest, there are two general categories of interest within the current psychological literature: situational interest and individual interest. Situational interest is influenced by the environment an individual is in at the time, such as a classroom, and may or may not last over time. Individual (or personal) interest refers to the type of long-lasting interest that is less influenced by the environment. This type of interest forms over time and focuses on an individual's inner development of interest. 
The four-phase model views interest from a development standpoint and describes four sequential and cumulative phases in interest development involving situational and individual interest: triggering situational interest, maintained situational interest, emerging individual interest, and well-developed individual interest (Hidi \& Renninger, 2006). Triggering situational interest is the first phase of interest development. An individual's interest is peaked when something within their environment triggers their interest, such as when Steve discussed engaging with chemistry after realizing the realworld applications. This engagement could then lead to the second phase of interest development; maintained situational interest. In the maintained situational interest phase, a student may become interested in course content based on a previous trigger and begin to re-engage with material. Within this phase, interest is sustained based on meaningfulness of tasks, such as Nancy and Max when they described applying their knowledge to something new. The third phase is emerging individual interest, which is categorized by stored knowledge, values, and positive feelings. Within this phase, interest is mostly self-generated. For example, a student may begin to increasingly value their interaction with the course material based on previous engagement and will continue to re-engage with the material. This was illustrated by Robin, when she described integrating science into her "real life" to understand how things work. It is also possible that Robin was in the final phase of interest development; well-developed individual interest. This phase is categorized by more stored knowledge, value, and positive feelings than emerging individual interest. Similar to emerging individual interest, well-developed individual interest is mostly self-generated. An individual will seek out extra 
opportunities to re-engage with the subject even if faced with setbacks within this phase. For example, a student may choose to take a non-required course despite a non-ideal grade in a previous chemistry course.

Based on the four-phase model of interest, the appropriate measure of interest depends on the population under study. Within the Carlone and Johnson (2007) research, interest was not studied because the participating women were in their late undergraduate years and early career and it was assumed that their interest in science was established. This established phase of interest aligns with the emerging or well-developed phases of individual interest. Hazari and colleagues (2010) studied a population that was earlier within their undergraduate career and not solely STEM majors. Triggered or maintained situational interest may have aligned more appropriately with this population, as the particular students may have been more prone to changes in interest based on their classroom environments. Designing a measure to target specific phases of interest can provide more detailed information on what phase of interest is the most impactful on identity formation. Situational interest is the most malleable phase of interest and could be an important construct to target when studying identity formation within classroom environments.

\section{Theme 2: Educational experiences contribute to student science or chemistry identity.} Students made it clear that educational experiences pertaining to science and chemistry were a crucial component of science and chemistry identity. Representative student quotes can be found within Table 4.8. Within the Attributes and Self-Recognition section of the interview, Elle described a chemistry person as someone who does well in class, Steve described a chemistry person as being careful in the course laboratory, and 
Max described herself as a science person because of success in the classroom. These statements provided evidence that students were drawing on experiences within the classroom or laboratory to describe science and chemistry people rather than experiences with science and chemistry outside of the classroom.

Students continued to draw on educational experiences to relate to science and chemistry within the Performance/Competence section. For example, when asked generally about setbacks in science or chemistry, students frequently mentioned setbacks in the classroom such as poor grades or repeating a course. Examples come from Erica, who had to overcome poor exam grades, and Barb, who repeated courses because of poor course grades. When asked about confidence in science or chemistry, students mentioned their performance in school or knowledge of science or chemistry content as the basis of their confidence. For example, Karen described doing well on an exam for the basis of her confidence. Educational experiences in reference to identity were also present within the Recognition section. Erica and Karen both described their experiences performing well within the subject as a way that others recognized them as science or chemistry people.

Throughout the interviews, students described educational experiences as a source of science or chemistry identity. This theme aligned with Carlone and Johnson's original definitions of performance and competence (Figure 4.1A) because students were directly describing their competency and performance in their education pertaining to science or chemistry when discussing identity. They did not explicitly mention confidence when unprompted, but instead described their mastery experiences, such as taking an exam, 
when responding to questions that inquired about performance/competence or attributes of a science or chemistry person. This theme differed slightly from the definition provided by the physics identity framework of performance/competence, where the construct was described as being reflective of student confidence. This evidence suggested that describing performance/competence in the form of mastery experiences instead of confidence may be more appropriate.

Table 4.8. Student quotes pertaining to educational experiences in relation to science or chemistry identity.

\begin{tabular}{|c|c|}
\hline Student & Quote \\
\hline Elle & $\begin{array}{l}\text { "Having good study habits [makes someone a chemistry person]...You have to know all } \\
\text { the formulas, and how to apply the problems to those." }\end{array}$ \\
\hline Steve & $\begin{array}{l}\text { "You gotta be really careful with what you're doing, especially in chemistry lab. O-Chem } \\
\text { lab was the most stressful thing in my life. It's just making sure you're paying attention to } \\
\text { the instructions or making sure you're not putting the wrong things together and keeping } \\
\text { track of it all." }\end{array}$ \\
\hline Max & $\begin{array}{l}\text { "I mean I've always been good at science I think it's something that I can be successful } \\
\text { and I've always gotten mostly A's and a couple B's in any scientific area..." }\end{array}$ \\
\hline Erica & $\begin{array}{l}\text { “...I'll bomb my first exam pretty bad, about 50... And that's when I study really hard. } \\
\text { And the pattern is, my second exam, I usually get more than } 90 . . . \text { Yeah, I think that's } \\
\text { definitely a setback. It pushes me way behind, so then I have to go ahead and study old } \\
\text { stuff and the new stuff, 'cause it's cumulative." }\end{array}$ \\
\hline Barb & $\begin{array}{l}\text { "...The reason why I was taking biology last term and I'm taking chemistry now is 'cause } \\
\text { I'm retaking them from community college. So I just didn't really do as well as I wanted } \\
\text { to when I was in community college in those classes." }\end{array}$ \\
\hline Karen & $\begin{array}{l}\text { "Gen-chem was, I was really scared going into it so I studied really hard for the first exam } \\
\text { and then did obscenely well...." }\end{array}$ \\
\hline Erica & "I think they think I do well in it, so they [think I'm a science person]." \\
\hline Karen & "[My friends see me as a chemistry person] mainly because I was really good at it." \\
\hline
\end{tabular}

\section{Theoretical Framework Surrounding Theme 2}

Mastery Experiences

The construct of mastery experiences is a source of self-efficacy and was reflected

in student responses when they described the contribution of their educational

experiences to their science or chemistry identity. Self-efficacy is defined as "people's

judgments of their capabilities to organize and execute courses of action required to attain 
designated types of performances”(Bandura \& National Inst of Mental Health, 1986, p. 391). Social cognitive theory (SCT) emphasizes that perceived self-efficacy is directly correlated to behavioral change and is the foundation of human agency. When applied in a student-centered context, SCT implies that self-efficacy guides students' actions which in turn can influence their motivation and affect.

Mastery experiences are experiences in which an individual is able to base their self-perception on successes and failures of specific tasks. This is the most influential source of self-efficacy because it provides the clearest evidence to whether an individual can succeed in future tasks (Bandura, 1997, p. 80). An example of a mastery experience within the classroom is a student taking an exam. Exams occur multiple times within a course, providing students with the opportunity to learn and improve the outcomes in subsequent attempts. Several students mentioned mastery experiences, such as exams, or passing courses, when discussing their science or chemistry identity.

\section{Theme 3: Students gain information about identity through interactions with others.}

Students frequently mentioned interactions with others when discussing science or chemistry identity within the Recognition portion of the interview. Representative student quotes can be found within Table 4.9. There were three distinct ways that students described interacting with others as a form of recognition. First, students discussed interacting with others to negotiate their own identity through comparison of themselves with others. Erica compared herself to non-specific "others" who she saw as more or less advanced than herself in relation to chemistry. Nancy compared herself to her family when explaining why they recognize her as a science person. Steve compared himself to his peers when explaining how he isn't recognized as a chemistry person. 
Next, students mentioned being recognized by others when being approached for help or discussing science or chemistry in conversation. This was illustrated by Karen when she described her family approaching her for medical advice and Nancy when her friends asked her a question and she gave an in-depth response. Finally, students described being recognized, or not being recognized, as a science or chemistry person through encouragement or discouragement from others. Elle described her family verbalizing encouragement whereas Nancy described her peers verbalizing discouragement. Recognition by others as a science or chemistry person was dependent on interactions with others and this theme described multiple ways that those interactions occur. Two of these interactions, comparison to others and encouragement, aligned with the theoretically established constructs of vicarious experiences and verbal persuasion, respectively.

Table 4.9. Student quotes pertaining to their interactions with others to navigate their science or chemistry identity.

\begin{tabular}{|c|c|}
\hline Student & Quote \\
\hline Erica & $\begin{array}{l}\text { "There's clearly people who are far more advanced than I am, and then there's people } \\
\text { who are far behind. And I'm kind of in the middle, where I see myself in terms of } \\
\text { chemistry." }\end{array}$ \\
\hline Nancy & $\begin{array}{l}\text { "I'm the only person in my family that actually is like, "Science!" And even all my little } \\
\text { siblings are like, either no school or sociology." }\end{array}$ \\
\hline Steve & $\begin{array}{l}\text { "...I was like the only person in our study group who got a C and I was really bummed } \\
\text { because it's such an important class...like my other friends in my study group are just } \\
\text { chemistry geniuses.” }\end{array}$ \\
\hline Karen & $\begin{array}{l}\text { "I'm pre-med and...my family will literally just text me and be like, 'ey, this is going on } \\
\text { with me. What's wrong with me?'” }\end{array}$ \\
\hline Nancy & $\begin{array}{l}\text { "I always come up with weird quirky facts. Like, we'll be having a regular conversation and } \\
\text { they'll say something like trans-fatty acid or something, and then they'll be like, 'Well why } \\
\text { is that bad for you?' And then I'll be like, 'Well trans is like this,' and it's like, 'And then cis } \\
\text { is, '..." }\end{array}$ \\
\hline Elle & "My mom, and my brother, and my dad have all said, 'No, you're good at science." "' \\
\hline Nancy & $\begin{array}{l}\text { "[My peers are] like, 'Oh, maybe you're not that good at this. How are you gonna get } \\
\text { through it?'," }\end{array}$ \\
\hline
\end{tabular}




\section{Theoretical Framework Surrounding Theme 3}

Verbal Persuasion and Vicarious Experience

Verbal persuasion and vicarious experiences are two additional sources of selfefficacy. "People who are persuaded verbally that they possess the capabilities to master given tasks are likely to mobilize greater sustained effort than if they harbor self-doubts and dwell on personal deficiencies when difficulties arise" (Bandura, 1997, p. 101). Encouragement or discouragement from others as a form of recognition within the identity interviews aligned with verbal persuasion, as others were verbally encouraging or discouraging students to participate in science or chemistry. The other source of selfefficacy, vicarious experiences are used to judge an individual's self-efficacy based on watching others perform tasks. For many specific tasks, there are no absolute measures of adequacy. For example, if a student receives a score on an exam that is not a perfect score, they may not know if they performed adequately unless they compare scores with other students, as seen by Steve when he compared his grade of a "C" to other students within his study group.

There were multiple forms of recognition students described within the interviews. The established constructs of vicarious experiences and verbal persuasion aligned well with two forms of recognition. Targeting specific sources of recognition could be helpful in providing information on how to foster recognition and therefore science or chemistry identity.

\section{Theme 4: Participation in science or chemistry takes a certain type of person.}

The previous three themes contained additional information about interest, performance/competence, and recognition as described by the physics identity framework. This final theme was present throughout the interview but did not directly 
align with any of the previously described constructs. During interviews, students described science or chemistry people in two ways; those who had an innate talent for science or chemistry and those who were determined to succeed. Representative student quotes can be found within Table 4.10. Within the Attributes and Self-Recognition section, students would describe being a science or chemistry person as someone who has some type of trait that allows one to understand science or chemistry. For example, Max described a science person as someone with the ability to understand science and Elle described herself as lacking the ability to understand chemistry. By describing science and chemistry identities this way, students were describing who is equipped to be a science or chemistry person and inferred that it is an inherent trait. The second way students described a science or chemistry person was through hard work or determination. For example, within the Attributes and Self-Recognition section, Robin described that it takes grit and commitment to be a science person and Steve described a science person as someone who is driven to ask and answer questions about life. The two ways that students described certain types of people as being able to participate in science or chemistry aligned with the affective constructs of fixed and growth mindset.

Table 4.10. Student quotes pertaining to the types of people who participate in science or chemistry.

\begin{tabular}{ll}
\hline Student & Quote \\
\hline Max & $\begin{array}{l}\text { "Someone who's good at visualizing things that they can't see or understanding those } \\
\text { concepts." }\end{array}$ \\
\cline { 2 - 2 } Elle & $\begin{array}{l}\text { "I think my brain just isn't equipped to be able to deal with those kinds of [chemistry] } \\
\text { problems, or concepts." }\end{array}$ \\
\cline { 2 - 2 } Robin & $\begin{array}{l}\text { "I don't think it's a special skill per se, it's more about grit, just committing to } \\
\text { [science]." }\end{array}$ \\
\cline { 2 - 2 } Steve & $\begin{array}{l}\text { "It's kinda like, 'How do I work? Or how do we work?' So it's just having that drive to } \\
\text { figure stuff out." }\end{array}$ \\
\hline
\end{tabular}




\section{Theoretical Framework Surrounding Theme 4 \\ Mindset}

Growth mindset is the belief that one has the ability to develop their intelligence (Dweck, 2013). The antithesis of growth mindset is fixed mindset, which is the belief that intelligence is fixed and cannot be developed. While determination is not directly interchangeable with growth mindset, it is an important part of growth mindset (Dweck, 2015). Students such as Robin and Steve mentioned drive and commitment. While this doesn't confirm that Robin and Steve had a growth mindset, it did suggest that they did not have a fixed mindset. In comparison, Max and Elle described a science or chemistry person as having a certain ability that helps them to understand science or chemistry. This suggested that a person has an inherent trait within them and suggested more of a fixed mindset. Mindset interventions have been shown to increase performance in underachieving students and are theorized to aid in persistence in academia (Claro, Paunesku, \& Dweck, 2016; Dweck, 2009; Paunesku et al., 2015). Fixed vs. growth mindset may not have appeared in Carlone and Johnson's (2007) science identity study because the women involved in the study were further along in their science careers. While mindset hasn't explicitly been discussed as an aspect of identity, it may be an important variable to consider in future studies of science and chemistry identity.

\section{Summary}

In response to research question one, four themes arose when students responded to questions pertaining to science and chemistry identity. These themes were then discussed in terms of their relation to other, more theoretically grounded, constructs in order to address research question two. The established constructs, with their definitions aligned to science and chemistry identity, are listed in Figure 4.2. Three of the four 
themes provided clarification to the descriptions of interest, performance/competence, and recognition as described by the physics identity framework. Within the interviews, interest was discussed in terms of students' feelings and values and their relation to science or chemistry. This description of interest aligned with the theory that interest has both feeling- and value-related components (Schiefele, 1991). In addition to feelings and value, students described different stages of interest, which supported the four-phase model of interest (Hidi \& Renninger, 2006). Based on these alignments, the construct of interest was redefined as situational interest, where the orientation of students to science or chemistry was discussed through value- and feeling-related interest. Students discussed recognition in the terms of interactions with others. Two ways that these interactions occurred were through verbal persuasion and vicarious experiences (Bandura, 1977). Recognition was therefore divided into the two constructs of verbal persuasion and vicarious experiences. Verbal persuasion was defined as verbal recognition for being good at science or chemistry. Vicarious experiences were defined as recognition of self through comparison of others' experiences with science or chemistry. Performance/competence was discussed in terms of success within educational experiences. The types of successes described, e.g., course and exam grades, aligned with mastery experiences (Bandura, 1977). Mastery experiences were defined as experiences in which an individual is able to base their self-perception on successes and failures of specific tasks within the classroom. Within the fourth theme, students described science or chemistry people as either having an inherent trait that oriented them toward science or chemistry or as being determined to succeed within science or chemistry. This theme 
aligned with the theory of mindset (Dweck, 2013), where becoming a science or

chemistry person is either dependent on an inherent trait or attained through

determination. By exploring themes of identity formation and grounding them within

theoretically sound constructs, we have taken the first step in creating a measure of

identity to use within chemistry education.

SCIENCE IDENTITY

(Carlone and Johnson 2007)
PHYSICS IDENTITY

(Hazari et. al., 2010)

\section{SCIENCE/CHEMISTRY IDENTITY}

\section{Mindset}

Becoming a science or chemistry person is either dependent on an inherent trait

or is attained through determination

\section{Situational Interest}

Interest

Desire/curiosity to think about and understand physics
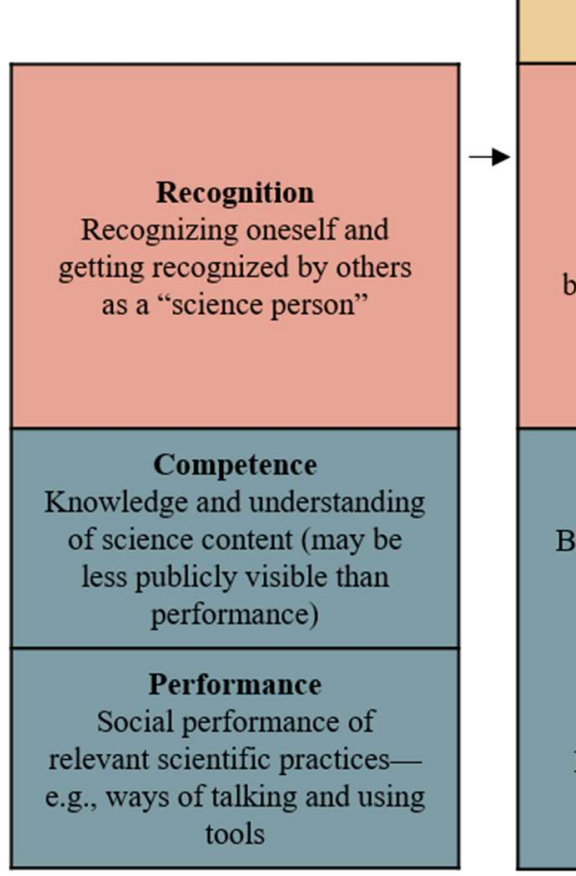

\section{Recognition}

Recognition by others as being a good physics student

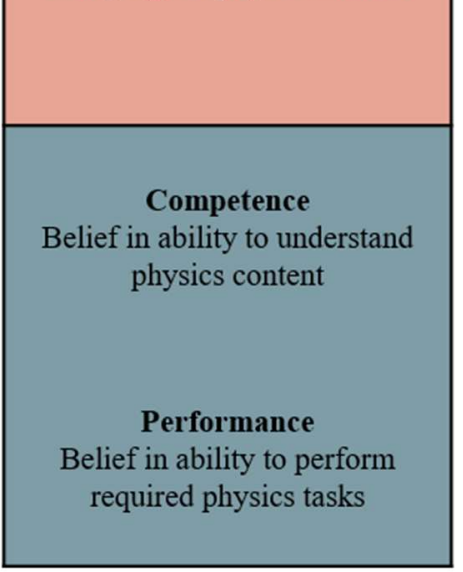

The orientation of a student to science or chemistry through value- and feeling-related interest

Verbal Persuasion Verbal recognition for being good at science or chemistry

Vicarious Experiences Recognition of self through comparison of others' experiences with science or chemistry

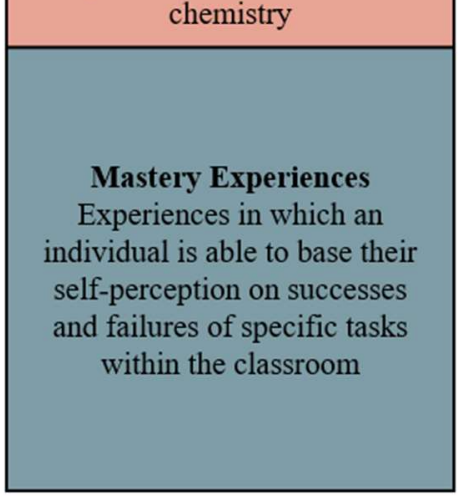

Figure 4.2. The alignment and modification of identity theories. 


\section{Conclusions and Limitations}

Identity has been theorized to be an important factor in student persistence (Chang et al., 2011; Estrada et al., 2011; Flowers \& Banda, 2016; Graham et al., 2013;

President's Council of Advisors on Science and Technology, 2012). However, to assess identity it first needs to be contextualized and well defined. This study built upon the work of Hazari and colleagues (2010) by further investigating the sub-constructs of the physics identity framework: interest, recognition, and performance/competence. Student interviews based on prior conceptualization of these constructs elicited responses that were aligned with the constructs of situational interest, verbal persuasion, vicarious experiences, mastery experiences, and mindset. Developing and collecting information reflecting these theoretically grounded constructs could potentially provide a more precise understanding of identity. By rooting the identity sub-constructs within these psychological constructs, we have taken the first step to creating a measure that can inform students' science or chemistry identity formation. In the future, having specific construct measures will provide insight to specific target variables for identity interventions.

The sources of self-efficacy have been previously hypothesized to align with science identity, further supporting the alignment of performance/competence with mastery experiences and recognition with verbal persuasion and vicarious experiences. Flowers and Banda (2016) have argued that these sources of self-efficacy are a crucial component for cultivating a science identity, specifically among minority students. Cultivating a strong self-efficacy for tasks within science can help students to believe they can be successful in the field of science. Vicarious experiences were mentioned as the source of 
self-efficacy missing from Carlone and Johnson's (2007) original science identity theory and is proposed to be a large contributor to science identity for students of color.

There were a few limitations within this study. First, the distinction between science and chemistry identity was not investigated. While there were no differing themes between wording when students responded to questions about both science and chemistry identity, future studies should explore the nuances between the two identities. Next, student interviews took place at one university with limited demographics. While evidence of data saturation was present within this sample, interviewing students at multiple universities or with more diverse demographics would provide additional support for the constructs of science and chemistry identity defined within this study. Qualitative studies provide rich data that can aid in the development or elucidation of theory, but alone, do not provide evidence for generalization. The next step in providing more robust support for a science or chemistry identity framework would be to distribute surveys containing the constructs found within the study to test their psychometric functioning. Finally, mastery experiences, vicarious experiences, and verbal persuasion were not discussed as a source of self-efficacy within the interviews but rather a direct source of identity. Future work should explore whether these constructs are direct sources of identity or if they are mediated by self-efficacy. 


\title{
Chapter 5
}

\section{Development and Evaluation of Novel Science and Chemistry Identity Measures}

\begin{abstract}
Identity has been proposed as a mechanism to increase persistence within Science, Technology, Engineering and Mathematics (STEM) education programs. To assess the impact of identity on STEM persistence, measures that produce valid and reliable data within a given STEM discipline need to be employed. Therefore, this study developed and evaluated the functioning of science and chemistry identity measures in the context of university-level chemistry courses. The developed measures were administered to students enrolled in general and organic chemistry courses at six universities across the United States. Validity and reliability evidence for the data provided by the novel measures was supported using confirmatory factor analysis and McDonald's omega. Additionally, two competing structural equation models (SEMs), designed to explore the relations between mastery experiences, verbal persuasion, situational interest, and science or chemistry identity, were tested and compared to previously reported results. Both SEMs produced acceptable model fit, therefore a superior model was chosen based on theoretical support. Within both SEMs, the direct pathway (relation) between mastery experiences and identity was nonsignificant. The more supported model proposed that the relation was indirect and facilitated through verbal persuasion and situational interest. While the indirect relation was supported in both courses, the predominate pathway varied by course. Limitations of the science identity measure and future recommendations for use of the Measure of Chemistry Identity (MoChI) are discussed.
\end{abstract}




\section{Introduction}

The fact that a majority of incoming Science, Technology, Engineering and Mathematics (STEM) students do not graduate with a STEM degree has been documented for some time. A report published in 2012 (President's Council of Advisors on Science and Technology, 2012) noted that less than $40 \%$ of students in the United States who began college with the intent of majoring in a STEM discipline actually obtained a STEM degree. To improve the retention of STEM students, the Council outlined five overarching recommendations, the first of which called for the "widespread adoption of empirically validated teaching practices (p. 16)." The report notes that, “classroom approaches that engage students in 'active learning' improve retention of information and critical thinking skills, compared with sole reliance on lecturing, and increase persistence of students in STEM majors (p. 17).” A proposed mechanism to increase persistence within STEM majors includes the fostering of students' STEM identities (Chang et al., 2011; Estrada et al., 2011; Flowers \& Banda, 2016; Graham et al., 2013; Shedlosky-Shoemaker \& Fautch, 2015).

Within this study, identity is defined as being recognized as a certain "type of person" in a specific context (Gee, 2000). Therefore, science identity is conceptualized as being recognized as a "science person" in a science context, such as a classroom. Chemistry identity is similarly conceptualized as being recognized as a "chemistry person" in a chemistry context. In order to assess changes in students' science or chemistry identity, we need measures that have been shown to produce valid and reliable data (Furr \& Bacharach, 2008). While there are several measures of various STEM identities (Cass et al., 2011; Chemers et al., 2011; Estrada et al., 2011; Godwin, Potvin, 
Hazari, et al., 2013; Hazari et al., 2010; Stets et al., 2017; Vincent-Ruz \& Schunn, 2018), they have not been operationalized specifically to identity as a "science or chemistry person" or are operationalized to disciplines other than chemistry.

When an instrument is developed to measure a psychological attribute, such as identity, the data provided by that instrument needs to show evidence of validity and reliability, which account for systematic and random error, respectively. Validity evidence can be provided through multiple sources including content validity, structural validity, response process validity, and relations with other variables (Furr \& Bacharach, 2008). While it is always necessary to provide evidence of data validity, the sources should match the intended use of a measure (American Educational Research Association et al., 2014). Reliability evidence can be provided through various means including testretest and single administration reliability estimates such as alpha and omega (Komperda, Pentecost, et al., 2018) and should be provided every time an instrument is used within a new sample.

To design a STEM identity measure, each construct involved in identity formation needs to be framed by an appropriate theory and well defined within that theory. These provide the basis for content and structural validity (Furr \& Bacharach, 2008). Carlone and Johnson (2007) described a science identity framework consisting of three subconstructs: recognition, performance, and competence (Figure 5.1A). To operationalize identity within physics, the physics identity framework (Hazari et al., 2010) built upon and modified the science identity framework and described three sub-constructs of physics identity: interest, recognition, and performance/competence (Figure 5.1B). 
Hosbein and Barbera (2020) built upon the physics identity framework to operationalize science and chemistry identity, aligning the sub-constructs of identity with mindset, situational interest, verbal persuasion, vicarious experiences, and mastery experiences. While alignment of the framework to science and chemistry identity has been explored through qualitative methods, the newly aligned sub-constructs have not been quantitatively investigated.

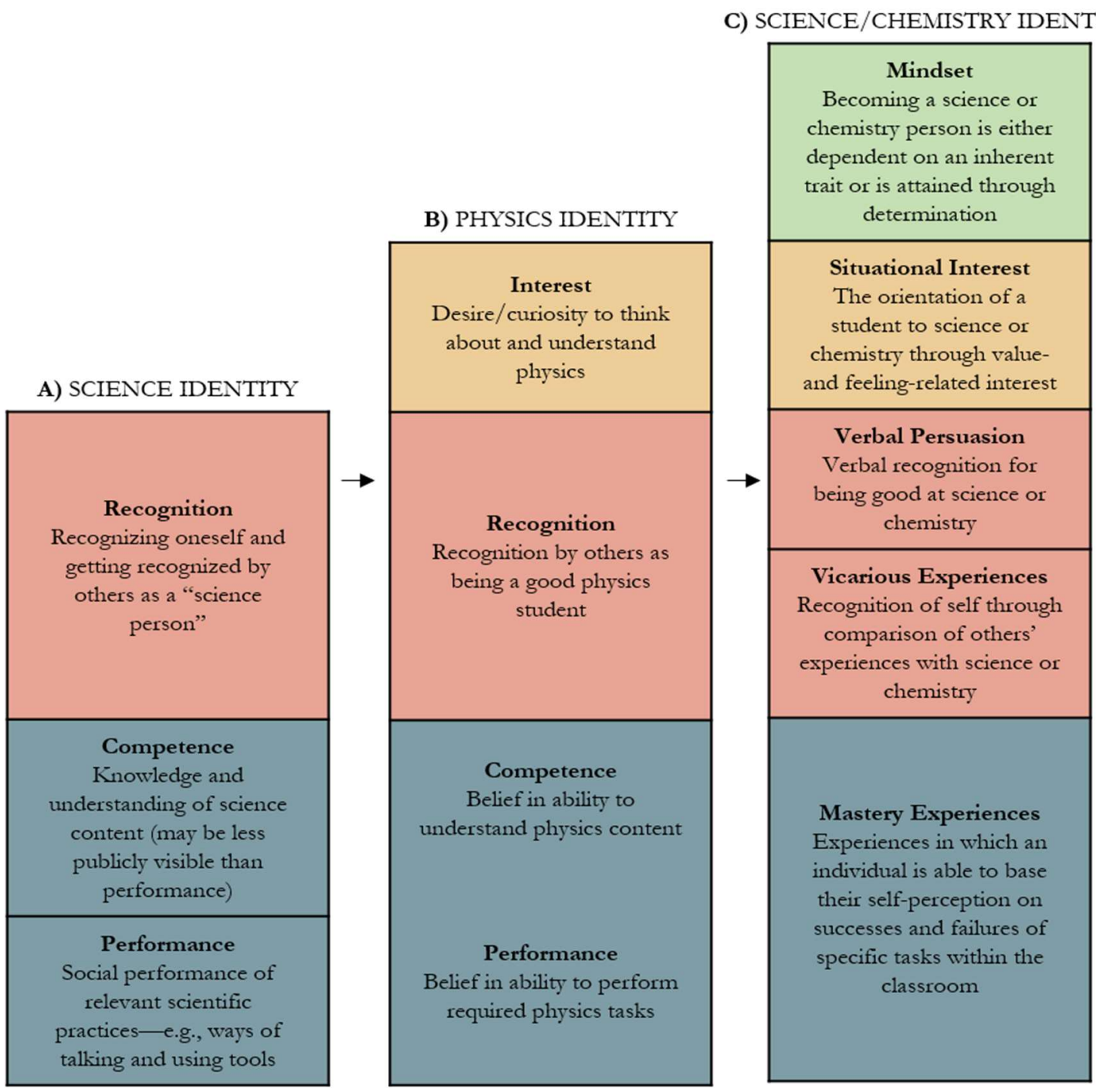

Figure 5.1. Affective constructs proposed to have an important role in identity formation as proposed by A) Carlone and Johnson (2007), B) Hazari and colleagues (2010), and C) Hosbein and Barbera (2020). 


\section{Theoretical Framework}

The alignment between the sub-constructs of STEM identities can be seen in the trajectory within Figure 5.1A to 5.1C. Carlone and Johnson (2007) described science identity as containing the sub-constructs of recognition, competence, and performance (Figure 5.1A). The sub-constructs of the physics identity framework (Figure 5.1B) built upon the original theory of science identity proposed by Carlone and Johnson (2007) in order to operationalize the theory within a specific discipline. Three distinct modifications were made between the sub-constructs. First, interest was added to the physics identity theory. Interest was described by Carlone and Johnson (2007) as important to identity formation but was not a part of their study because their sample consisted of women who were later in their career and thought to have a stable interest. The physics identity framework was applied to students early on in their career where interest may not be as stable and was therefore added to the framework (Hazari et al., 2010). Second, recognition was re-conceptualized in the physics identity framework. Self-recognition was viewed as a core feature to identity that could be influenced by recognition from others. Therefore, it was separated from the sub-constructs and used as a holistic identity variable consisting of a single item that asked, "Do you see yourself as a physics person?” (Potvin \& Hazari, 2013; Shanahan, 2009). Third, performance and competence were re-defined to reflect confidence in ability (similar to self-efficacy), rather than purely ability, and combined into a single construct based on structural validity evidence. The sub-constructs described in the physics identity framework were defined as interest, recognition, and performance/competence but they lacked grounding within theoretically framed affective constructs. These sub-constructs were further 
explored qualitatively in order to investigate their alignment with theoretically supported constructs and to operationalize them to science and chemistry identity (Hosbein \& Barbera, 2020). To provide quantitative support of the alignment between the physics identity framework (Figure 5.1B) and the science and chemistry identity constructs (Figure 5.1C), those directly aligned will be discussed (i.e., situational interest with interest, verbal persuasion with recognition, and mastery experiences with performance/competence).

\section{Relations between identity constructs}

The constructs of interest, recognition, and performance/competence have been hypothesized to play a role in identity development within the physics identity framework (Figure 5.1B). Their relations have been explored in prior studies through structural equation modeling (SEM) for math (Cribbs et al., 2015), physics (Godwin, Potvin, Hazari, et al., 2013), and science (Godwin, Potvin, Hazari, et al., 2013) identities. SEM is a family of related measurement methods that can provide information about causal inference. Testable models are constructed from a priori relations grounded in theory. While SEM is a method of causal inference, it does not provide an absolute model of causal relations between variables. Instead, it can be used to provide support for a theory described in the literature and the causal relations described by that theory (Kline, 2016, p. 11).

The sub-constructs of physics identity have been used within an SEM framework to explore relations between performance/competence, recognition, and interest with an identity indicator item. This identity indicator consists of a single item, "I see myself as a [ ] person", where the brackets have been replaced with discipline terms such as physics 
(Godwin et al., 2016; Hazari et al., 2010), math (Cass et al., 2011; Cribbs et al., 2015), and science (Godwin, Potvin, Hazari, et al., 2013). A baseline model (Figure 5.2A) was originally tested with performance/competence, recognition, and interest correlated to each other and each predicting identity. Alternatively, Cribbs and colleagues (2015) hypothesized that "competency beliefs (i.e., performance/competence) might precede and facilitate other perceptions that explain an individual's identity development (p. 1056).” To test this hypothesis, an alternative model (Figure 5.2B) was tested with an indirect relation between performance/competence and identity, facilitated through interest and recognition (Cribbs et al., 2015). This alternative model had improved model fit when compared to the baseline model and has been used in subsequent studies (Cheng et al., 2018; Godwin et al., 2016).

A)

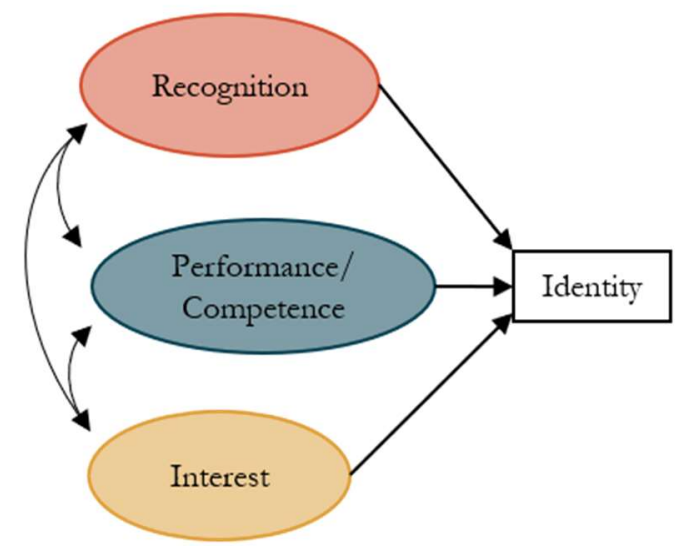

B)

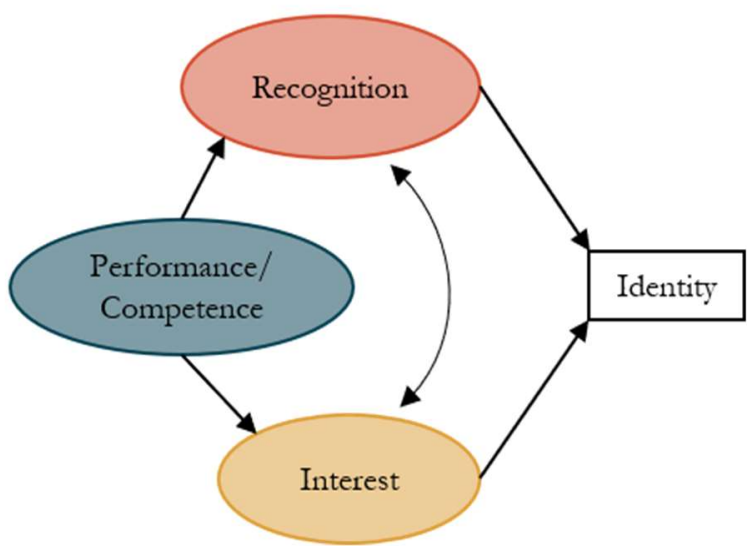

Figure 5.2. A) Baseline and B) alternative models with proposed relations between recognition, performance/competence, interest, and identity based on the physics identity framework. Indicator items and endogenous variable disturbances are omitted from the models for clarity.

In a recent qualitative study, Hosbein and Barbera (2020) proposed alignment between the constructs described in the physics identity framework (Figure 5.1B) and mastery experiences, verbal persuasion, and situational interest (Figure 5.1C). Support for 
these constructs and their relations to the alternative model lie within the theories of Social Cognitive Theory (SCT) (Bandura \& National Inst of Mental Health, 1986), situational interest (Hidi \& Renninger, 2006), and the science identity theory as proposed by Carlone and Johnson (2007). Within SCT, "When people aim for master valued levels of performance, they experience a sense of satisfaction (Locke, Cartledge, \& Knerr, 1970). The satisfactions derived from goal attainments foster intrinsic interest (Bandura \& National Inst of Mental Health, 1986, p. 242).” This statement provides support that one's mastery experiences may precede their interest. Additionally, within the four-phase theory of interest (Renninger \& Hidi, 2011, p. 170), situational interest has been shown to be marginally impacted by an individual's knowledge and values, providing further support that mastery experiences (equated to knowledge in this case) may precede interest. The relation of interest with verbal persuasion is indirectly described within SCT, “...interest grows from satisfactions derived from fulfilling challenging standards and from self-percepts of efficacy gained through accomplishments and other sources of efficacy information (Bandura \& National Inst of Mental Health, 1986, p. 243). " This suggests that verbal persuasion could precede interest, however, the relation is not explicitly described and could be correlational. This description within SCT also provides further support of the directional relation between mastery experiences and interest. When describing the relation between verbal persuasion and mastery experiences, directionality is not specified within SCT, however, there is support for directionality within the context of science identity theory. An indirect effect of mastery experiences on identity through verbal persuasion is supported in the description of identity by Carlone 
and Johnson (2007); a person performs tasks that illustrate their competence in a way that an individual is recognized by others as a credible science person. While some support exists for directional relations between constructs, some relations are not explicitly described as directional. Therefore, it is unclear whether the constructs share correlational, or directional relations. Hence, both the baseline and alternative models (Figures $2 \mathrm{~A}$ and $\mathrm{B}$ ) will be modified to include mastery experiences, verbal persuasion, and situational interest and the relations tested.

\section{Purpose and Rationale for the Study}

Prior to conducting any SEM testing, the psychometric functioning of each construct measure must be established. Therefore, the first aim of this study was to analyze the selected measures of the sub-constructs of science and chemistry identity. While science identity has been measured on undergraduate populations, it may be more appropriate to target discipline-specific identities when focusing on higher education classroom environments. Affective measures using science and discipline-specific wording have been shown to function differently depending on wording and class type (Glynn et al., 2011; Komperda, Hosbein, \& Barbera, 2018; Salta \& Koulougliotis, 2015). While the minor wording change of "science" to "chemistry" may seem insignificant, validity evidence is required to support that the measure functions equally in both wording versions. Additionally, exploring any changes in science or chemistry identity as a result of changes in classroom practice is dependent on an instrument that has been shown to function within each wording type and environment under study. Additional support for the interpretation of the data provided by the measures comes from the use cognitive interviews to establish evidence of response process validity. 
The second aim of this study was to model the relations between mastery experience, verbal persuasion, situational interest to further explore how these constructs contribute to the formation of science or chemistry identity. The alignment between the sub-constructs of identity as described by the physics identity framework (Figure 5.1B) and the sub-constructs of science and chemistry identity (Figure 5.1C) have been previously explored qualitatively (Hosbein \& Barbera, 2020). To further support the alignment, their quantitative relations need to be investigated and compared to the previously explored relations of the physics identity framework (Figure 5.2). The two aims were carried out through the following research questions:

3) To what degree will an instrument containing items designed to measure mastery experiences, verbal persuasion, and situational interest show psychometric functionality in undergraduate chemistry courses with

a) science-specific wording?

b) chemistry-specific wording?

4) What are the relations between mastery experiences, verbal persuasion, situational interest and a

a) science identity indicator?

b) chemistry identity indicator?

\section{Methods}

\section{Survey Participants}

After obtaining institutional review board (IRB) approval for the study, chemistry instructors at six different United States universities were contacted to aid in recruitment. The recruitment sample consisted of students enrolled in undergraduate chemistry 
courses at three Northwestern universities, a Southwestern university, and two Midwestern universities, selected through convenience sampling. To sample a range of student levels (e.g., by major and year in degree), the selected courses included organic and general chemistry targeted toward science majors. A total of 855 organic and 2,324 general chemistry students were recruited for participation in the study.

\section{Instrument Scales}

Mastery Experiences and Verbal Persuasion

The mastery experience and verbal persuasion scales were adapted from the

Sources of Middle School Mathematics Self-Efficacy Scale (Usher \& Pajares, 2009). The instrument was developed in response to the lack of a measure targeted toward the four sources of self-efficacy (mastery experiences, verbal persuasion, vicarious experiences, and physiological state) in middle school mathematics. While this instrument also contained a vicarious experiences scale, a construct shown to align with recognition (Hosbein \& Barbera, 2020), it was not used because vicarious experiences is an indirect form of recognition and therefore verbal persuasion was more appropriately aligned with recognition for the purpose of this study. Items for the four sources of were developed according to SCT (Bandura \& National Inst of Mental Health, 1986), which encompasses the theory of self-efficacy. Scales aligned with these sources were iteratively developed over three rounds of data collection and psychometric analysis. Although the measure was tailored in wording for middle school students, the theory was rooted in SCT (with items reviewed by Bandura himself) and items on the measure mirrored already existing sources of self-efficacy measures developed in a college setting (Fencl \& Scheel, 2003; Lent et al., 1996). For this reason, as well as the support provided by the psychometric 
analysis performed on the measure, the verbal persuasion and mastery experience subscales from the Sources of Middle School Mathematics Self-Efficacy scale were chosen. Each scale contained six items on a six-point Likert-type response scale ranging from definitely false to definitely true.

\section{Situational interest}

The initial and maintained interest scales developed by Ferrell and Barbera (2015) were used in this study to measure situational interest. The two measures were originally operationalized in psychology (Harackiewicz et al., 2008) and adapted to a general chemistry context (Ferrell \& Barbera, 2015). Both initial and maintained interest measures were chosen in order to capture the interest of students at the beginning and end of a course. Both measures contained items from two constructs: feeling- and valuerelated interest (Schiefele, 1991). Feeling-related interest items were tied to emotional arousal and value-related items were tied to importance/utility. The initial interest measure contained seven items; four feeling- and three value-related interest items. The maintained interest measure contained eight items; four feeling- and four value-related interest items. All items were on five-point Likert-scale ranging from strongly disagree to strongly agree with a "neutral" response.

\section{Scale modifications}

Items on all scales were duplicated to create separate "science" and "chemistry" versions. Items that did not use either phrase were not duplicated. Wording changes were made to the items on each scale, as needed, to reflect the constructs of science and chemistry, specifically in an undergraduate course setting. For example, the mastery experience and verbal persuasion scales were originally operationalized for middle school 
mathematics. To modify these items, the word "math" was modified to "science" and "chemistry". Additionally, items containing words in the context of middle school were changed to reflect undergraduate courses. For example, the item "I got good grades in math on my last report card" was modified to "I have gotten good course grades in [ ]", where the bracket is replaced with chemistry or science to create each version. The initial and maintained interest scales were originally operationalized specifically for general chemistry; therefore, for the purpose of using the scale in multiple undergraduate chemistry courses, items were modified to remove this specificity. For example, the item "I think that what we will study in General Chemistry will be important for me to know" was modified to "I think that what we will study in this class will be important for me to know". All original and corresponding modified items are included in Table B.1.

The mastery experience and verbal persuasion response scales were modified from a six-point Likert-type scale to a five-point Likert scale to align with the interest scales and for the purpose of including a neutral mid-point within the scale. The authors of the Sources of Middle School Mathematics Self-Efficacy Scale did not provide an argument for the even number of response choices on their Likert-type scale. While it is unadvisable to include a mid-point in a Likert-scale for measures that contain items that could be socially undesirable because participants may select a neutral option to avoid responding honestly to the item (Johns, 2005), the mastery experience and verbal persuasion scales do not have items that could be deemed socially undesirable and therefore a mid-point was included to capture participants who had a legitimate neutral response to the items. Additionally, the change in wording of the original response scale, 
"definitely false" to "definitely true", to the Likert response scale "strongly agree" to "strongly disagree" did not change the meaning of any of the items after review by the authors.

\section{Identity Indicator}

Identity within this study was conceptualized as "seeing oneself as a science person in a science context" and "seeing oneself as a chemistry person in a chemistry context". Prior studies that have used the physics identity framework have measured identity using a single indicator item as a holistic measure for modeling purposes(Cass et al., 2011; Cribbs et al., 2015; Godwin, Potvin, Hazari, et al., 2013; Godwin et al., 2016; Verdín et al., 2018). Therefore, the identity indicator of "I see myself as a [ ] person", where the brackets were replaced with either science or chemistry, was used in this study, for the purpose of modeling the sub-construct relations to science or chemistry identity.

\section{Survey Data Collection}

Survey distribution occurred during the first and last weeks of the courses during

Fall 2018. Students were recruited by the lead author through an in-class announcement delivered through a recorded video or in person. The survey was hosted on the website Qualtrics and the link to the survey was posted to the respective course websites after each in-class announcement. The first survey (time 1) contained the mastery experiences, verbal persuasion, initial feeling- and value-related interest, and the identity indicator. In the final survey (time 2), the initial interest scales were replaced with the maintained interest scales. Demographics were collected at the end of the time 1 survey. Both "science" and "chemistry" versions of each scale and identity indicator were included on the survey with a five-point Likert scale at both time points. Each "science" item was 
immediately followed by its "chemistry" item counterpart. This was done so that students would directly compare their responses for the chemistry and science wording of the survey. Items that did not use either term only appeared once, as there was not a counteritem. Each of the items and linked pairs were randomized for each student to avoid any item order effects in the data.

\section{Cognitive Interview Participants}

After obtaining IRB approval, a portion of the population who participated in the

time 1 survey were selected for cognitive interviews through convenience sampling during the Winter 2019 term at one Northwestern university. Students from two sections of both general and organic chemistry courses were recruited through an in-class announcement and email. A total of 381 and 536 students were recruited for cognitive interviews in organic and general chemistry, respectively. Students did not receive compensation for their participation.

\section{Cognitive Interview Data Collection}

Response process validity is used to provide evidence that the items on an instrument are being interpreted in the intended way by the sample under study (Krosnick \& Presser, 2010). To provide this evidence, cognitive interviews are often used (Arjoon et al., 2013). At the beginning of each cognitive interview, students were provided with a copy of the survey items from the verbal persuasion, mastery experiences, initial feelingrelated interest, and initial value-related interest scales. Only the initial interest scales were provided because the interviews occurred within the first half of the term and therefore maintained interest of the course may not have been formed. Students only saw one wording-type, either science or chemistry. Students read each question aloud and 
were asked three questions: "What is this item trying to find out from you?", "Which response would you choose as the right response for you?", and "Can you explain to me why you chose that response?" Follow up questions were asked for additional clarification as necessary.

\section{Data Analysis}

Data Cleaning

Only students who completed both time 1 and time 2 measures were included in the dataset. A self-check item was included in the survey that read "Please select 'Disagree' for this item”. Students who selected a response other than 'Disagree' were removed from the dataset. Missing data was deleted based on wording type responses. For example, if a student had completed all of the science-worded items at times 1 and 2, but did not complete an item within the chemistry wording at time 1 , their chemistry responses from both times 1 and 2 were removed and science responses retained.

Responses from the time 1 and time 2 measures were then matched. After matching complete datasets, duplicated responses were removed based on the self-reported name of the respondents. If duplicated responses were present, the first entry was retained. Descriptive Statistics and Response Patterns

Student responses to time 1 and time 2 measures were examined between wording and course types. Descriptive statistics including mean, standard deviation, median, range, skew, and kurtosis were computed. Acceptable skew and kurtosis values were between -1 and 1 (Huck, 2012). All descriptive statistics were computed using the statistical software R (Version 3.4.4). 


\section{Confirmatory Factor Analysis}

A structural equation model (SEM) contains two components: measurement and structural. The measurement component of the model consists of the indicator items and scales used to measure latent variables. The structural component of the model consists of the proposed relations between the measured latent variables. Before a full SEM is investigated, the measurement portion of the model needs to be tested (Mueller \& Hancock, 2008). This was done through confirmatory factor analysis (CFA). CFA was chosen over exploratory factor analysis (EFA) because the scales used were based on strong a priori hypothesis for their factor structure (Kline, 2016, pp. 190-191). CFAs were performed using R (Version 3.4.4) and the lavaan package (Version 0.5-23.1097). One-, two-, and multi-factor measurement models for time 1 and time 2 data were tested independently. One-factor models were used to justify reliability estimates that require unidimensional scales (Komperda, Pentecost, et al., 2018) as well as to test measurement invariance over time (Newsom, 2015). Two two-factor models were evaluated (feelingwith value-related interest and mastery experiences with verbal persuasion) to mimic previous analysis of these scales within the literature (Ferrell \& Barbera, 2015; Usher \& Pajares, 2009), providing additional evidence for their functioning in the new contexts. Multi-factor models were tested to provide support for the full SEM analysis (Mueller \& Hancock, 2008). In all models, the science and chemistry worded items were separated and tested. To complete each data set, the unduplicated items (i.e., those that contained the phrase "this class" instead of "science" or "chemistry") were included in both versions during analysis. 
Normality in the data distributions was assessed to determine the appropriate CFA estimator. Continuous data is an assumption of the maximum likelihood estimators and while the Likert-scale technically provides ordinal data, the scale can be thought of as continuous when it contains five or more response options and is approximately normally distributed(Dolan, 1994). In evaluating appropriate model fit, three fit indices and a standard set of cutoff values were utilized: $\mathrm{CFI} \geq 0.95$, RMSEA $\leq 0.06$, and $\mathrm{SRMR} \leq$ 0.08 (Hu \& Bentler, 1999). Modification indices were used to determine if post-hoc modifications of each model were necessary.

Reliability

Single-administration reliability is commonly reported using the statistic alpha. While this may be appropriate for certain types of data, omega is more appropriate to report when describing models with unequal item error variances and unequal factor loadings, known as congeneric models (Komperda, Pentecost, et al., 2018). All models within this study were evaluated as congeneric models and therefore omega was used to provide a reliability estimate. Omega, like alpha, requires that a scale is unidimensional, therefore it was only calculated for an individual scale (mastery experience, verbal persuasion, feeling-, and value-related initial and maintained interest) if evidence of adequate one-factor CFA model fit was obtained. Omega values mirror alpha values in their range from 0 to 1 with higher values indicating higher reliability. When latent variables are included in SEM, it is recommended that their reliability estimates fall above 0.7 (Hancock \& Mueller, 2001). Although there are R packages to calculate omega (Komperda, Pentecost, et al., 2018), they do not take into account item error correlations 
present within the model. Omega values were calculated by hand using Equation 5.1 to incorporate item error correlations when necessary,

$$
\omega=\frac{\left(\sum_{i=1}^{J} \lambda_{i}\right)^{2}}{\left(\sum_{i=1}^{J} \lambda_{i}\right)^{2}+\sum_{i=1}^{J} \theta_{i}+2 \sum_{i=1}^{J} \theta_{i j}}
$$

where $\lambda_{\mathrm{i}}$ is item factor loading, $\theta_{i}$ is item variance, and $\theta_{i j}$ is the variance associated with the item error correlation.

\section{Structural Equation Modeling}

After measurement models were tested through CFA, the relations between constructs could be investigated through full SEMs (Figure 5.3). The SEMs were built upon the previously proposed models shown in Figure 7. First, recognition was replaced by verbal persuasion, performance/competence was replaced by mastery experiences, and interest was replaced by both feeling- and value-related interest components. Next, the models were modified to reflect the effects of the measures at time 1 on the corresponding measures at time 2 . To do this, autoregressive pathways were included between time 1 and time 2 constructs. Autoregressive pathways account for the direct effect of a variable on itself over time, for example, the effect of mastery experiences at time 1 on mastery experiences at time 2 . Finally, maintained interest is a construct that develops over time and therefore, students were not expected to come into the course with maintained interest. To account for this, initial feeling- and value-related interest was measured at time 1 as a control for maintained feeling- and value-related interest measured at time 2. Although the model in Figure 5.2B does not have a direct pathway shown between mastery experiences and identity, this pathway was tested (Cribbs et al., 
2015) in the original study using the model and was found to be nonsignificant. To re-test this pathway, it was included in the alternative model in Figure 3.
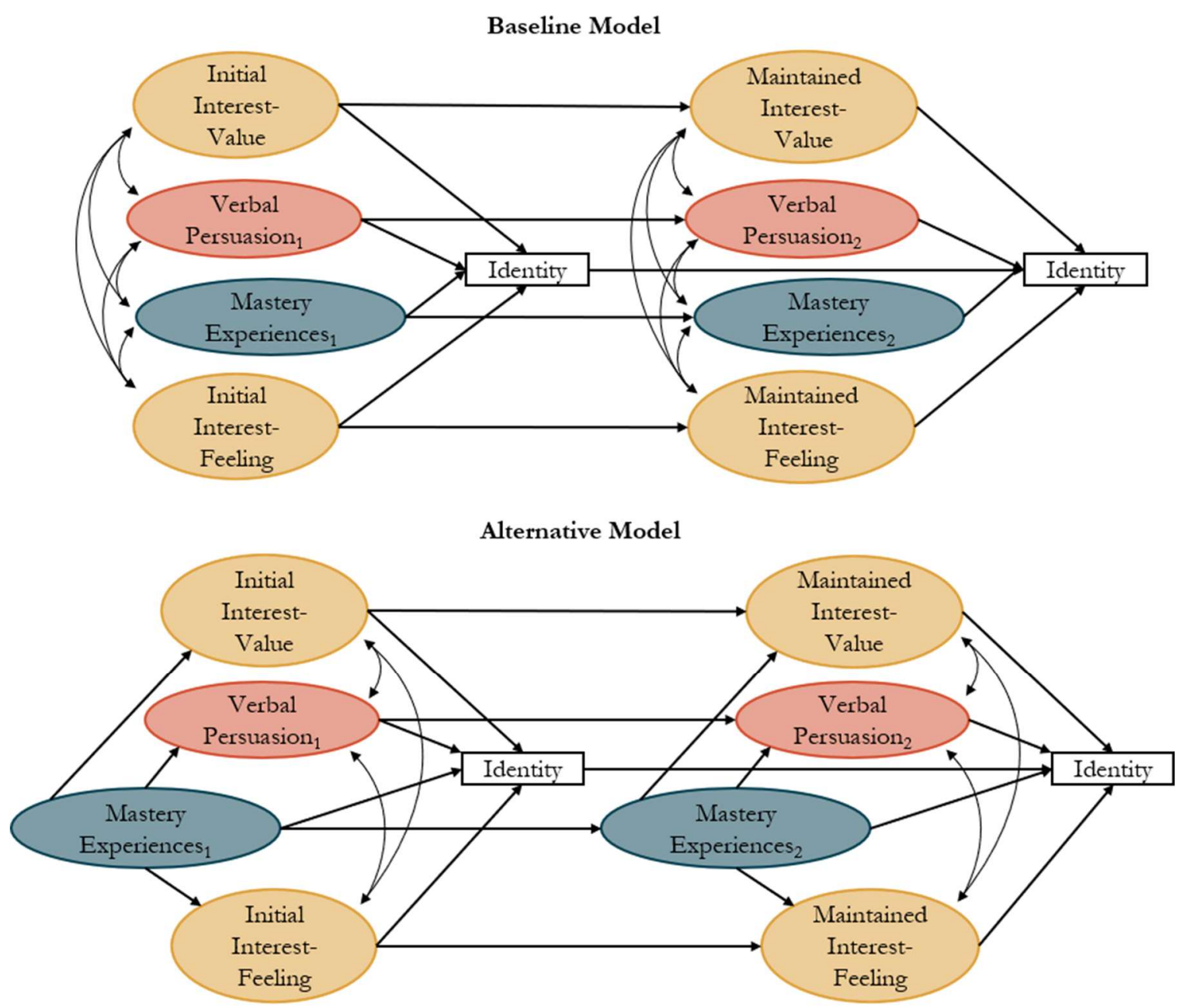

Figure 5.3. Proposed baseline and alternative models for SEM. Indicator items and endogenous variable disturbances are omitted for clarity.

Before testing the full SEMs, it was necessary to show evidence of longitudinal invariance, i.e., that the repeated measures from times 1 and 2 were measuring the same construct. There are various levels of invariance testing. The lowest level of invariance is configural invariance, where the measures within each group (in this case mastery experiences and verbal persuasion) are shown to be invariant without holding any 
parameter equal between groups (time 1 and time 2) (Fischer \& Karl, 2019). The next highest level of invariance is metric invariance, where measures are shown to be invariant when the factor loadings are held equal between groups. Higher levels of invariance entail holding additional parameters equal between groups, such as intercepts and latent means. It is recommended to at least provide evidence of metric invariance between repeated measures before testing a full SEM model (Newsom, 2015). Metric invariance testing was completed for the repeated measures of mastery experience and verbal persuasion for both wording types within each course. Models provided evidence of invariance if the change in chi-square was nonsignificant between configural and metric invariant models (Newsom, 2015). After showing evidence of metric invariance for repeated measures, the full SEMs within Figure 5.3 were tested for each wording type within each course. To compare SEM parameters between general and organic chemistry courses within each wording type, multi-group metric invariance must be tested (Fischer \& Karl, 2019). Multi-group invariance was determined by a nonsignificant change in chisquare (Cheung \& Rensvold, 2002). All SEM analyses were carried out through the lavaan package (Version 0.5-23.1097) in R (Version 3.4.4). Normality in the data distributions was assessed to determine the appropriate SEM estimator. After estimation, cutoff values indicating good model fit followed that of the CFA models: CFI $\geq 0.95$, RMSEA $\leq 0.06$, and SRMR $\leq 0.08$.

Cognitive Interview Analysis

Cognitive interviews were used to assess the readability and interpretation of survey items for response process validity (Arjoon et al., 2013). Evidence for response process validity had been previously shown in the development of each measure (Ferrell 
\& Barbera, 2015; Usher \& Pajares, 2009) but with slightly different samples. However, the process was repeated here as an assurance that the slight wording changes of each item did not alter the meaning. Positive evidence was provided when a majority of the students' interpretation of an item aligned with the intended meaning of the item. If student interpretation misaligned with the intended meaning, that item was noted and marked for further review by the authors.

\section{Results and Discussion}

Cognitive Interviews

A total of eight students participated in a cognitive interview; four from general chemistry and four from organic chemistry. Within each course type, two students responded to the science worded version of the measures while two students responded to the chemistry version of the measures. No students had issues reading any particular item aloud. Items were then evaluated for appropriate responses. For example, when a student was responding to a verbal persuasion item, it would be expected that they would discuss verbal feedback from others. A specific example included the item "My chemistry instructors have told me that I'm good at chemistry" where a student who disagreed responded, "This is more like if an instructor thinks I'm good enough and singles me out and says 'hey you're good at this'... I don't think that's ever happened." The student here selected "disagree" as a response and the explanation corresponded to correct scale usage and interpretation of the item. None of the items were found to prompt inappropriate student responses within the interviews. This was expected based on the previous response validity evidence provided by the measures in their development. 


\section{Survey Data Cleaning}

Responses in the final dataset included those that passed four criteria: 1) the

student was 18 years or older and selected to consent to the research study, 2) the student selected 'Disagree' for the check item, 3) the student completed all of the chemistryworded or all of the science-worded items on the survey, and 4) the student completed both time 1 and time 2 surveys. Data cleaning was performed using R (Version 3.4.4). After cleaning, there were 1,198 responses; 676 in general chemistry (335 science worded responses and 341 chemistry worded responses) and 522 in organic chemistry (225 science worded responses and 226 chemistry responses).

\section{Survey Participant Characteristics}

General chemistry participants were mostly white (60\%), female (65\%), biology majors (38\%) or other science majors (33\%) with an average age of $21 \pm 1.6$ years. Organic chemistry participants were mostly white (73\%), female (67\%), biology majors $(46 \%)$ or other science majors (23\%), with an average age of $22 \pm 1.4$ years.

\section{Descriptive Statistics and Response Patterns}

Mean, standard deviation, median, minimum, maximum, skew, and kurtosis were computed for all items on the time 1 and time 2 surveys. These descriptive statistics can be found in Tables B.2 and B.3. The means of the items from the time 1 survey ranged from 2.89 to 4.78 . Means of the items from the time 2 survey ranged from 2.95 to 4.28. The science-worded items had higher means for all items as compared to the chemistryworded items. Within the time 1 survey, students utilized the entire magnitude of the Likert scale for all items except for the three items on the initial value-related interest scale, two items on the initial feeling-related interest scale, and one mastery experience item. Students utilized the entire magnitude of the Likert scale for all items on the time 2 
survey. Most items contained skew and kurtosis within the acceptable range of -1 to 1 , however, some fell outside of this range with skew values as low as -1.96 and kurtosis values as high as 3.07 .

\section{Confirmatory Factor Analysis}

Based on the skew and kurtosis values of some of the items, the maximum likelihood estimator with the Satorra-Bentler adjustment and robust standard errors was used as the estimator in all CFA models. If any non-normality is present within the data, it can be treated by using maximum likelihood with Satorra-Bentler adjustment with robust standard errors (Satorra \& Bentler, 1994).

\section{Two-Factor Models}

Eight correlated two-factor CFA models were tested, feeling- with value-related interest and mastery experiences with verbal persuasion for both wording and course types and time 1 and 2. All scales were slightly modified in order to operationalize them to non-specific college chemistry courses. To ensure that the scales were functioning as

intended, two-factor models were chosen based on the previous use of the scales (Ferrell \& Barbera, 2015; Usher \& Pajares, 2009) and to provide an initial check for any measurement error before moving to a multi-factor CFA model containing all scales.

\section{Interest Scales}

When analyzing the two-factor initial feeling- and value-related interest scales, modification indices suggested correlations between some of the items present on the time 1 survey. Four of the seven initial interest items contained the phrase "this class", for example, "I chose to take this class because I'm really interested in the topic." The remaining three items either contained the word "chemistry" or "science", for example, "I am really looking forward to learning more about [science].” The modification indices 
suggested correlations between the three items containing the word "science". It is likely that when students read these items, they equated "this class" with "chemistry", as the survey was given in chemistry courses, but students may not necessarily respond to the item in the context of their class when presented with the "science" wording. Results from the cognitive interviews further supported this modification. An example of a student response to "I think chemistry is important", included, "I think it's really important because chemistry is used to solve a whole bunch of stuff. And like I don't know-see how chemical reactions work-figure out how things are made and how things work" which described content that a student would learn within their chemistry course. Alternatively, when students responded to the item "I think science is important", they mentioned science outside of the classroom, such as, "It makes me think about, "is science an important part of the world?' is it valuable to you, or do you perceive it as valuable to everybody" and, "I think science is one of the most important jobs there is." Based on the modification indices and qualitative support, the errors of the items containing the word "science" were correlated within the initial interest scales for subsequent models.

With the noted error correlations, the two-factor initial feeling- and value-related interest time 1 survey models had acceptable model fit with the exception of the RMSEA index for both wording types within general chemistry and the chemistry wording within organic chemistry (Table 5.1). When the selected indices are not in agreement, joint criteria can be used to assess acceptable fit (Mueller \& Hancock, 2008). The joint criteria included a SRMR $\leq 0.09$ with either a CFI $\geq 0.96$ or RMSEA $\leq 0.06$. The initial feeling- 
and value-related interest two-factor models fell within the joint criteria range for all wording and course types. Fit indices for time 2 maintained feeling- and value- related interest two-factor models suggested adequate fit for all wording and course types (Table $5.1)$.

Table 5.1. Model fit for correlated two-factor feeling- and value-related interest models with error correlations. Acceptable model fit indices in bold (CFI $>0.95$, RMSEA $<0.06$, SRMR $<0.08)$. Values within models providing acceptable fit through joint criteria $(\mathrm{SRMR}<0.09$ and CFI $>0.96$.

\begin{tabular}{|c|c|c|c|c|c|c|c|c|}
\hline Scales & Course $^{a}$ & Wording $^{b}$ & $\mathrm{df}$ & $\chi^{2}$ & CFI & RMSEA & $\begin{array}{l}\text { RMSEA } \\
{[90 \% \mathrm{CI}]}\end{array}$ & SRMR \\
\hline \multirow{4}{*}{$\begin{array}{l}\text { Initial feeling- and } \\
\text { value-related } \\
\text { interest } \\
\text { (Time } 1 \text { survey) }\end{array}$} & \multirow{2}{*}{$\mathrm{GC}$} & $\mathrm{S}(\mathrm{N}=335)$ & 10 & $30.4 *$ & 0.98 & 0.09 & {$[0.05,0.13]$} & 0.04 \\
\hline & & $\mathrm{C}(\mathrm{N}=341)$ & 13 & $34.5^{*}$ & 0.98 & 0.08 & {$[0.05,0.11]$} & 0.04 \\
\hline & \multirow{2}{*}{$\mathrm{OC}$} & $\mathrm{S}(\mathrm{N}=225)$ & 10 & 6.16 & 1.00 & 0.00 & {$[0.00,0.05]$} & 0.02 \\
\hline & & $\mathrm{C}(\mathrm{N}=226)$ & 13 & $35.7 *$ & 0.97 & 0.10 & {$[0.06,0.14]$} & 0.04 \\
\hline \multirow{4}{*}{$\begin{array}{l}\text { Maintained value- } \\
\text { and feeling- } \\
\text { related interest } \\
\text { (Time } 2 \text { survey) }\end{array}$} & \multirow{2}{*}{$\mathrm{GC}$} & $\mathrm{S}(\mathrm{N}=335)$ & 19 & 24.7 & 1.00 & 0.04 & {$[0.00,0.07]$} & 0.02 \\
\hline & & $\mathrm{C}(\mathrm{N}=341)$ & 19 & 33.6 & 0.99 & 0.06 & {$[0.02,0.09]$} & 0.03 \\
\hline & \multirow{2}{*}{$\mathrm{OC}$} & $\mathrm{S}(\mathrm{N}=225)$ & 19 & 33.9 & 0.98 & 0.07 & {$[0.03,0.10]$} & 0.03 \\
\hline & & $\mathrm{C}(\mathrm{N}=226)$ & 19 & 23.4 & 1.00 & 0.04 & {$[0.00,0.08]$} & 0.03 \\
\hline
\end{tabular}

Although both time 1 and time 2 interest models provided evidence of adequate fit, there were issues with localized fit for one item on the initial value-related interest scale. The item "I think [ ] is important." had low loading values of 0.41 and 0.27 for the science wording in the general and organic chemistry courses, respectively. In addition to the low loadings, the item had high means, skew, and kurtosis across both wording and course types ( 4.57 to $4.78,-1.96$ to -1.17 , and 0.77 to 3.07 , respectively). Due to these issues, the item was removed. However, after discarding the item, only two items remained on the initial value-related interest scale, which created an issue for further analysis using the scale as three or more items per factor are required for CFA modeling (Kline, 2016, p. 463). Therefore, the initial value-related interest scale was removed from further analysis. Additionally, the maintained value-related interest scale was also 
removed from further analysis as the control of initial value-related interest was no longer available. The initial and maintained feeling-related interest scales were then tested as one-factor CFA models for both wording and course types. These analyses were to ensure that the single-factor scale would function without the value-related interest component. Both one-factor models provided evidence of adequate fit according to cutoff criteria or joint cutoff criteria for both wording and course types (Table B.4).

Mastery experiences and verbal persuasion

When testing the correlated two-factor mastery experiences and verbal persuasion model, modification indices suggested error correlations between two pairs of mastery experience items. The first error correlation was between the item "I have been successful with [ ] in the past" and "I have gotten good course grades in [ ]" and the second error correlation was suggested between the items, "I do well on non-exam [ ] assignments" and "I do well on even the most difficult non-exam [ ] assignments". In the former pair, both items referenced past experiences with science or chemistry at the course level while the context of the remaining items inquired about present experiences at the exam level. The latter pair of items were very similarly worded and deemed redundant. The item "I do well on non-exam [ ] assignments" was removed from further analysis based on lower factor loadings within both wording and course types as compared to the alternative item. Item errors were correlated for the first pair and the two-factor CFAs were re-run. All subsequent model fit values (Table 5.2) suggested adequate fit when using selected cutoff criteria or joint criteria 
Table 5.2. Model fit for correlated two-factor mastery experience and verbal persuasion models with error correlation. Acceptable model fit indices in bold (CFI $>0.95$, RMSEA $<0.06$, SRMR $<0.08$ ). Values within models providing acceptable fit through joint criteria (SRMR $<0.09$ and CFI $>0.96$.

\begin{tabular}{|c|c|c|c|c|c|c|c|c|}
\hline Scales & Course $^{a}$ & Wording $^{b}$ & $\mathrm{df}$ & $\chi^{2}$ & CFI & RMSEA & $\begin{array}{l}\text { RMSEA } \\
{[90 \% \mathrm{CI}]}\end{array}$ & SRMR \\
\hline \multirow{4}{*}{$\begin{array}{c}\text { Mastery } \\
\text { experiences and } \\
\text { verbal persuasion } \\
\text { (Time } 1 \text { survey) }\end{array}$} & \multirow{2}{*}{$\mathrm{GC}$} & $\mathrm{S}(\mathrm{N}=335)$ & 42 & 63.6 & 0.99 & 0.05 & {$[0.02,0.03]$} & 0.03 \\
\hline & & $\mathrm{C}(\mathrm{N}=341)$ & 42 & 65.7 & 0.99 & 0.05 & {$[0.02,0.07]$} & 0.04 \\
\hline & \multirow{2}{*}{$\mathrm{OC}$} & $\mathrm{S}(\mathrm{N}=225)$ & 42 & $76.2^{*}$ & 0.96 & 0.07 & {$[0.04,0.09]$} & 0.05 \\
\hline & & $\mathrm{C}(\mathrm{N}=226)$ & 42 & $92.5^{*}$ & 0.96 & 0.08 & {$[0.06,0.10]$} & 0.06 \\
\hline \multirow{4}{*}{$\begin{array}{c}\text { Mastery } \\
\text { experiences and } \\
\text { verbal persuasion } \\
\text { (Time } 2 \text { survey) }\end{array}$} & \multirow{2}{*}{ GC } & $\mathrm{S}(\mathrm{N}=335)$ & 42 & 59.5 & 0.99 & 0.04 & {$[0.01,0.06]$} & 0.03 \\
\hline & & $\mathrm{C}(\mathrm{N}=341)$ & 42 & $94.9 *$ & 0.96 & 0.07 & {$[0.05,0.09]$} & 0.06 \\
\hline & \multirow{2}{*}{$\mathrm{OC}$} & $\mathrm{S}(\mathrm{N}=225)$ & 42 & 56.8 & 0.98 & 0.04 & {$[0.00,0.07]$} & 0.04 \\
\hline & & $\mathrm{C}(\mathrm{N}=226)$ & 42 & $76.1^{*}$ & 0.97 & 0.07 & {$[0.04,0.09]$} & 0.05 \\
\hline
\end{tabular}

\section{Three-factor models}

After providing evidence for acceptable fit for the correlated two-factor models of mastery experiences and verbal persuasion and single factor models of feeling-related interest, correlated three-factor models were tested to ensure the full measurement model provided adequate fit before moving to full SEMs. Any item error correlations present within previous models were retained. Fit indices for the correlated three-factor models (shown in Table 5.3) suggested adequate model fit for all wording and course types with the exception of the time 1 model consisting of the science wording within organic chemistry. The fit indices of 0.94, 0.07, and 0.09 for the CFI, RMSEA, and SRMR were just shy of the cutoff values. This was taken into consideration during further analysis as this misfit of the measurement model could contribute to misfit during SEM. Details including factor loadings and error terms for all correlated three-factor models are included in Figures B.1- B.4. 
Table 5.3. Model fit for correlated three-factor measurement models consisting of feeling-related initial or maintained interest, verbal persuasion, and mastery experiences. Acceptable model fit indices in bold (CFI $>0.95$, RMSEA < 0.06, SRMR < 0.08).

\begin{tabular}{|c|c|c|c|c|c|c|c|c|}
\hline Survey & Course $^{a}$ & Wording $^{b}$ & $\mathrm{df}$ & $\chi^{2}$ & CFI & RMSEA & $\begin{array}{l}\text { RMSEA } \\
{[90 \% \mathrm{CI}]}\end{array}$ & SRMR \\
\hline \multirow{4}{*}{ Time 1} & \multirow{2}{*}{ GC } & $\mathrm{S}(\mathrm{N}=335)$ & 85 & $142 *$ & 0.97 & 0.05 & {$[0.03,0.06]$} & 0.06 \\
\hline & & $\mathrm{C}(\mathrm{N}=341)$ & 86 & $149 *$ & 0.97 & 0.05 & {$[0.04,0.07]$} & 0.05 \\
\hline & \multirow{2}{*}{$\mathrm{OC}$} & $\mathrm{S}(\mathrm{N}=225)$ & 85 & $157^{*}$ & 0.94 & 0.07 & {$[0.05,0.08]$} & 0.09 \\
\hline & & $\mathrm{C}(\mathrm{N}=226)$ & 86 & $154^{*}$ & 0.96 & 0.07 & {$[0.05,0.08]$} & 0.06 \\
\hline \multirow{4}{*}{ Time 2} & \multirow{2}{*}{$\mathrm{GC}$} & $\mathrm{S}(\mathrm{N}=335)$ & 86 & 115 & 0.99 & 0.04 & {$[0.02,0.05]$} & 0.04 \\
\hline & & $\mathrm{C}(\mathrm{N}=341)$ & 86 & $168 *$ & 0.97 & 0.06 & {$[0.05,0.07]$} & 0.06 \\
\hline & \multirow{2}{*}{$\mathrm{OC}$} & $\mathrm{S}(\mathrm{N}=225)$ & 86 & 127 & 0.97 & 0.05 & {$[0.03,0.07]$} & 0.05 \\
\hline & & $\mathrm{C}(\mathrm{N}=226)$ & 86 & $151^{*}$ & 0.96 & 0.06 & {$[0.05,0.08]$} & 0.05 \\
\hline
\end{tabular}

${ }^{a} \mathrm{GC}=$ General Chemistry, $\mathrm{OC}=$ Organic Chemistry. ${ }^{b} \mathrm{~S}=$ Science, $\mathrm{C}=$ Chemistry. ${ }^{*} p \leq 0.001$

\section{Reliability}

Single-administration reliability was determined in the form of omega. Omega, like alpha, ranges from 0 to 1 , with 1 indicating that all of the observed variance is explained by the true construct variance. Unlike alpha, omega allows for unique factor loadings for each item, where alpha assumes equal factor loadings. Therefore, one-factor congeneric models were run for each scale with both wording and course types to provide evidence of unidimensionality and model fit before calculating omega(Komperda, Pentecost, et al., 2018). All models provided evidence for acceptable fit through cutoff criteria or joint model fit (Table B.4). Omega values are reported in Table 5.4, with all values above the recommended cutoff of 0.7 (Hancock \& Mueller, 2001). Values ranged from 0.74-0.92 for all scales in both courses for both wording types at times 1 and 2 . This range of omega values provided evidence that $74 \%$ to $92 \%$ of the observed variance was explained by the items measuring each individual construct (the true construct variance) (Komperda, Pentecost, et al., 2018). 
Table 5.4. Omega values for time 1 and time 2 one-factor models.

\begin{tabular}{|c|c|c|c|c|c|c|c|}
\hline \multirow[b]{2}{*}{ Course $^{a}$} & \multirow[b]{2}{*}{ Wording $^{b}$} & \multicolumn{3}{|c|}{ Time 1 Scales } & \multicolumn{3}{|c|}{ Time 2 Scales } \\
\hline & & $\begin{array}{l}\frac{\text { Initial }}{\text { Interest- }} \\
\underline{\text { Feeling }}\end{array}$ & $\frac{\text { Verbal }}{\text { Persuasion }}$ & $\begin{array}{c}\text { Mastery } \\
\text { Experiences }\end{array}$ & $\frac{\text { Maintained }}{\underline{\text { Interest- }}}$ & $\begin{array}{c}\frac{\text { Verbal }}{\text { Persuasion }} \\
\underline{\underline{y}}\end{array}$ & $\begin{array}{c}\text { Mastery } \\
\text { Experiences }\end{array}$ \\
\hline \multirow{2}{*}{$\mathrm{GC}$} & $\mathrm{S}$ & $0.76^{*}$ & 0.90 & $0.81^{*}$ & 0.89 & 0.90 & $0.79 *$ \\
\hline & $\mathrm{C}$ & 0.87 & 0.90 & $0.79 *$ & 0.90 & 0.91 & $0.79 *$ \\
\hline \multirow{2}{*}{$\mathrm{OC}$} & $\mathrm{S}$ & $0.74 *$ & 0.89 & $0.75^{*}$ & 0.88 & 0.89 & $0.78^{*}$ \\
\hline & $\mathrm{C}$ & 0.87 & 0.91 & $0.83 *$ & 0.92 & 0.90 & $0.80^{*}$ \\
\hline
\end{tabular}

$\overline{{ }^{a} \mathrm{GC}}=$ General Chemistry, $\mathrm{OC}=$ Organic Chemistry. ${ }^{b} \mathrm{~S}=$ Science, $\mathrm{C}=$ Chemistry. ${ }^{*}$ Omega value includes item error correlation errors.

\section{Structural Equation Modeling}

Multiple steps were taken to test the proposed structural equation models (SEMs)

displayed in Figure 5.4, choose the most appropriate model, and interpret the resulting SEM parameters for both wording and course types. First, longitudinal invariance was tested between mastery experiences and verbal persuasion at time 1 and time 2 . Next, the baseline and alternative models (Figure 5.4) were tested for model fit. Finally, multigroup invariance between general and organic chemistry models was tested in order to compare SEM parameters between wording types within each course.

\section{Longitudinal Invariance}

Mastery experiences and verbal persuasion were the only repeated measures.

One-factor models of mastery experiences at times 1 and times 2 were tested for metric invariance. This was repeated with one-factor verbal persuasion models. Fit indices for the metric invariance models are provided in Table 5.5. A nonsignificant change in chisquare between configural and metric invariance models provides evidence of metric invariance. When the Satorra-Bentler adjustment and robust standard errors are used in model estimation, a simple chi-square change cannot be calculated. Instead, chi-square change was calculated using an adjusted calculation to account for the alternative 
estimator (Satorra \& Bentler, 2010). Metric invariance was established for all but two of the one-factor models across time points for both course and wording types according to the nonsignificant chi-square change values (Table 5.5). The mastery experience scales with both wording types in general chemistry resulted in a significant chi-square difference. When the chi-square change is significant between nested models, the change in McDonald's Measure of Centrality (Mc) between the configural and metric invariance models can be used to measure the magnitude of non-invariance. Mc values were calculated using the R package ccpsyc (Version 0.2.1), which takes into account the Satorra-Bentler adjustments. Evidence for invariance is supported with a change in Mc $\leq$ 0.02 (Cheung \& Rensvold, 2002). The change in Mc for mastery experiences with science and chemistry wording were 0.007 and 0.02 respectively. Therefore, these changes in Mc between configural and metric invariance models provided evidence that the amount of non-invariance was small. In addition, the Mc values for the science and chemistry worded metric models were 0.99 and 0.97 , respectively. These values lie within the acceptable cutoff range off of Mc $\geq 0.96$ (Sivo, Fan, Witta, \& Willse, 2006). Therefore, the model fit of the metric invariance model of mastery experiences with both science and chemistry wording in general chemistry was deemed acceptable. After longitudinal invariance was investigated, full SEMs were tested. 
Table 5.5. Fit indices for metric invariance testing between correlated one-factor models at time 1 and time 2. Acceptable model fit indices in bold (CFI $>0.95$, RMSEA $<0.06$, SRMR $<0.08)$. Values within models providing acceptable fit through joint criteria

\begin{tabular}{|c|c|c|c|c|c|c|c|c|}
\hline Course $^{a}$ & Scale $^{b}$ & Wording $^{c}$ & $\Delta \chi^{2}{ }_{\text {sig }}{ }^{d}$ & $\chi^{2}$ & CFI & RMSEA & $\begin{array}{c}\text { RMSEA } \\
{[90 \% \mathrm{CI}]}\end{array}$ & SRMR \\
\hline \multirow{4}{*}{$\mathrm{GC}$} & Mastery & $\mathrm{S}(\mathrm{N}=335)$ & 0.00560 & $22.40 *$ & 0.99 & 0.06 & {$[0.01,0.10]$} & 0.05 \\
\hline & Experiences & $\mathrm{C}(\mathrm{N}=341)$ & $<0.001$ & $49.83 * *$ & 0.96 & 0.11 & {$[0.08,0.15]$} & 0.08 \\
\hline & Verbal & $\mathrm{S}(\mathrm{N}=335)$ & 0.135 & $50.77 * *$ & 0.98 & 0.07 & {$[0.04,0.10]$} & 0.04 \\
\hline & Persuasion & $\mathrm{C}(\mathrm{N}=341)$ & 0.0968 & 34.29 & 0.99 & 0.05 & {$[\mathrm{NA}, 0.08]$} & 0.04 \\
\hline \multirow{4}{*}{$\mathrm{OC}$} & Mastery & $\mathrm{S}(\mathrm{N}=225)$ & 0.0872 & $22.74 *$ & 0.98 & 0.08 & {$[0.02,0.12]$} & 0.05 \\
\hline & Experiences & $\mathrm{C}(\mathrm{N}=226)$ & 0.579 & 11.95 & 1.00 & 0.00 & {$[0.00,0.08]$} & 0.04 \\
\hline & Verbal & $\mathrm{S}(\mathrm{N}=225)$ & 0.520 & $45.76^{*}$ & 0.98 & 0.08 & {$[0.04,0.11]$} & 0.04 \\
\hline & Persuasion & $\mathrm{C}(\mathrm{N}=226)$ & 0.500 & $45.76^{*}$ & 0.98 & 0.09 & {$[0.06,0.12]$} & 0.05 \\
\hline
\end{tabular}

${ }^{a} \mathrm{GC}=$ General Chemistry, $\mathrm{OC}=$ Organic Chemistry. ${ }^{b}$ Degrees of freedom were 11 and 22 for mastery experiences and verbal persuasion models, respectively. ${ }^{c} \mathrm{~S}=$ Science, $\mathrm{C}=$ Chemistry. ${ }^{d} \Delta \chi^{2}$ sig $=$ significance of $\Delta \chi^{2}$ between configural and metric invariance models $* * p \leq 0.001, * 0.001 \leq p \leq 0.05$.

\section{Baseline and Alternative Model Testing}

Mastery experiences, verbal persuasion, and situational interest have been shown

to align with the constructs of performance/competence, recognition, and interest as proposed by physics identity framework (Hosbein \& Barbera, 2020). The previously tested baseline and alternative SEMs (Figure 5.2) proposed by Cribbs and colleagues, (2015) were modified to explore the relations between mastery experiences, verbal persuasion, and feeling-related initial and maintained interest with the identity indicator (Figure 5.4). The maximum likelihood estimator with the Satorra-Bentler adjustment and robust standard errors was used as the estimator in all SEMs due to the skew and kurtosis of multiple items. Baseline and alternative model fit indices for both wording and course types are contained in Table 5.6. All models showed acceptable model fit based on the cutoff criteria of all three fit indices or joint criteria cutoff ranges. 
Baseline Model
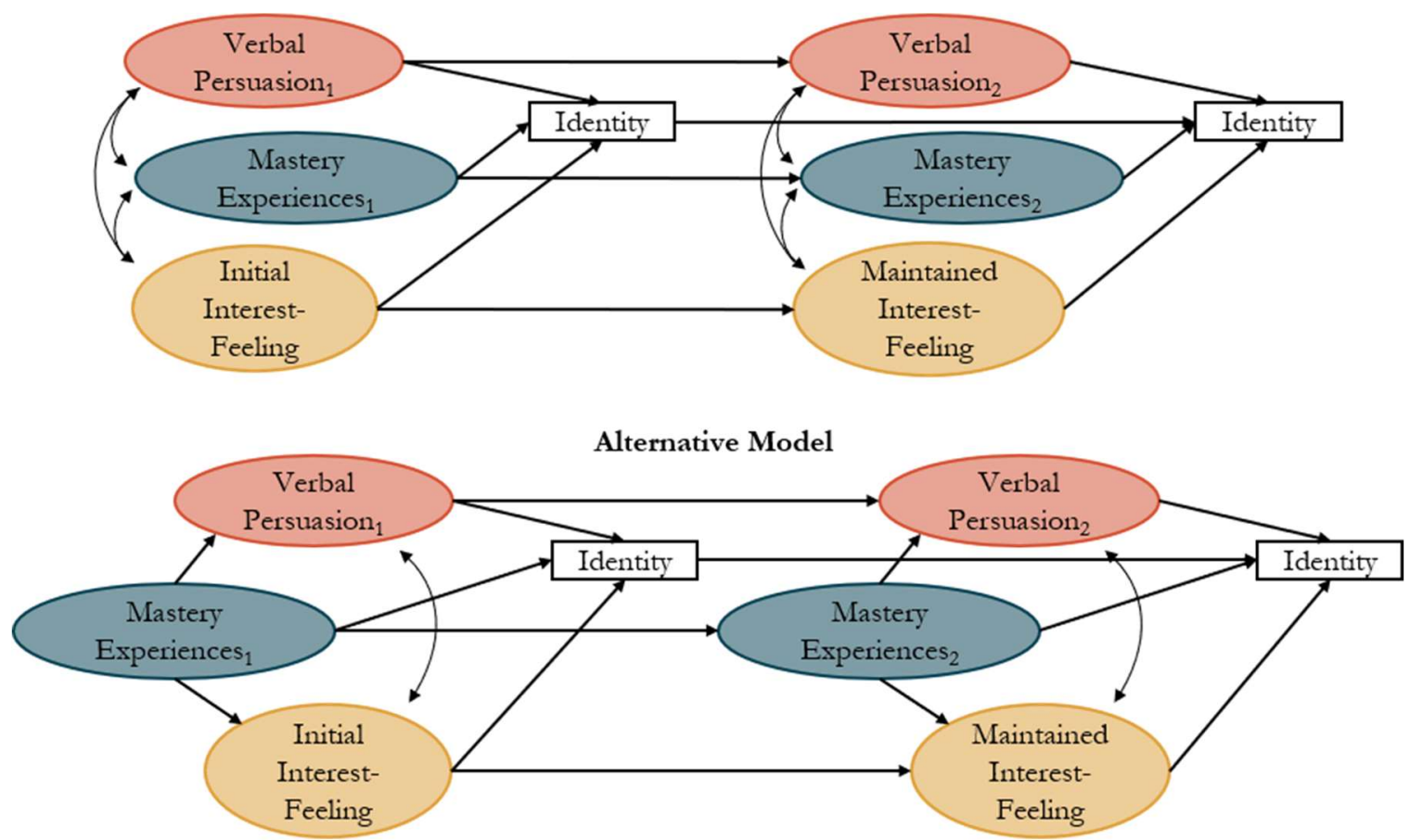

Figure 5.4. Baseline and alternative SEMs after the removal of initial and maintained value-related interest based on CFA results. Indicator items and endogenous variable disturbances are omitted for clarity.

Table 5.6. Model fit for baseline and alternative structural equation models. Acceptable model fit indices in bold $(\mathrm{CFI}>0.95$, RMSEA $<0.06$, SRMR $<0.08)$. Values within models providing acceptable fit through joint criteria $(\mathrm{SRMR}<0.09$ and $\mathrm{CFI}>0.96$

\begin{tabular}{|c|c|c|c|c|c|c|c|c|}
\hline Model & Course $^{a}$ & Wording $^{b}$ & df & $\chi^{2}$ & CFI & RMSEA & $\begin{array}{l}\text { RMSEA } \\
{[90 \% \mathrm{CI}]}\end{array}$ & SRMR \\
\hline \multirow{4}{*}{ Baseline } & \multirow{2}{*}{$\mathrm{GC}$} & $\mathrm{S}(\mathrm{N}=335)$ & 436 & $619^{*}$ & 0.97 & 0.04 & {$[0.03,0.05]$} & 0.06 \\
\hline & & $\mathrm{C}(\mathrm{N}=341)$ & 437 & $690 *$ & 0.96 & 0.05 & {$[0.04,0.05]$} & 0.06 \\
\hline & \multirow{2}{*}{$\mathrm{OC}$} & $\mathrm{S}(\mathrm{N}=225)$ & 436 & $658^{*}$ & 0.93 & 0.05 & {$[0.04,0.06]$} & 0.08 \\
\hline & & $\mathrm{C}(\mathrm{N}=226)$ & 435 & $666^{*}$ & 0.95 & 0.05 & {$[0.04,0.06]$} & 0.07 \\
\hline \multirow{4}{*}{ Alternative } & \multirow{2}{*}{$\mathrm{GC}$} & $\mathrm{S}(\mathrm{N}=335)$ & 438 & $659^{*}$ & 0.96 & 0.04 & {$[0.04,0.05]$} & 0.06 \\
\hline & & $\mathrm{C}(\mathrm{N}=341)$ & 437 & $708^{*}$ & 0.95 & 0.05 & {$[0.04,0.05]$} & 0.07 \\
\hline & \multirow{2}{*}{$\mathrm{OC}$} & $\mathrm{S}(\mathrm{N}=225)$ & 438 & $668^{*}$ & 0.93 & 0.05 & {$[0.04,0.06]$} & 0.08 \\
\hline & & $\mathrm{C}(\mathrm{N}=226)$ & 437 & $674 *$ & 0.95 & 0.05 & {$[0.05,0.06]$} & 0.07 \\
\hline
\end{tabular}

${ }^{a} \overline{\mathrm{GC}}=$ General Chemistry, $\mathrm{OC}=$ Organic Chemistry. ${ }^{b} \mathrm{~S}=$ Science, $\mathrm{C}=$ Chemistry. ${ }^{*} p<0.001$

It is possible for data to fit multiple proposed models. When this occurs,

theoretical backing can be used to choose the more acceptable model (Kline, 2016, pp.

10-11). Within the original baseline model (Figure 5.2), the direct pathway between 
performance/competence and identity was found to be nonsignificant (Cribbs et al., 2015). This prompted the development and testing of the alternative model. Although performance/competence wasn't directly related to identity, it was still thought to play a role in identity formation (Cribbs et al., 2015). While the alternative model shown in Figure 5.2 does not indicate the direct pathway, it was tested within the original study and shown to indeed be nonsignificant (Cribbs et al., 2015). The alternative model has been re-tested and shown to provide adequate model fit in additional studies with multiple wording-types (math, physics, and science) (Cheng et al., 2018; Godwin et al., 2016). The results from the baseline and alternative models in Figure 5.4 produced similar results, with the direct effect of mastery experiences on identity being nonsignificant for all baseline and alternative models. The alternative model's indirect pathway from mastery experiences to identity through recognition was supported by Carlone and Johnson's (2007) science identity theory, which states that a person performs tasks that illustrate their competence in a way that an individual is recognized by others as a credible science person. Additionally, the alternative model's indirect pathway between mastery experiences and identity through situational interest was supported by both Social Cognitive Theory (SCT) (Bandura \& National Inst of Mental Health, 1986) and situational interest as described by the four-phase model of interest (Hidi \& Renninger, 2006), which both describe satisfaction coming from mastery experiences or knowledge acquisition. Therefore, despite their equivalent model fit, the alternative model was more supported based on previous results (Cheng et al., 2018; Cribbs et al., 2015; Godwin et al., 2016) and theoretical backing by SCT (Bandura \& National Inst of Mental Health, 
1986), situational interest (Hidi \& Renninger, 2006), and science identity theory as proposed by Carlone and Johnson (2007).

Multi-Group Invariance

To compare SEM parameters between models, multi-group invariance must be established. This invariance was tested between the alternative models for general and organic chemistry data with the same wording type. The change in chi-square value between configural and metric models as well as the metric model fit indices are listed in Table 5.7. Both course models showed a nonsignificant change in chi-square as well as acceptable model fit. Taken together, these results allowed for comparison of model parameters between courses within the same wording type. The parameters between wording types could not be compared because metric invariance could not be evaluated between the models. This was due to the presence of error correlations within the science wording that were not present in the chemistry wording.

Table 5.7. Fit indices for full SEM model metric invariance testing between courses. Acceptable model fit indices in bold $(\mathrm{CFI}>0.95$, RMSEA $<0.06$, SRMR $<0.08)$.

\begin{tabular}{ccccccccc}
\hline Model & Wording $^{b}$ & df & $\Delta \chi^{2}$ sig $^{c}$ & $\chi^{2}{ }_{\text {metric }}$ & CFI & RMSEA & $\begin{array}{c}\text { RMSEA } \\
{[90 \% \mathrm{CI}]}\end{array}$ & SRMR \\
\hline $\begin{array}{c}\mathrm{S}\left(\mathrm{N}_{\mathrm{GC}}=335,\right. \\
\left.\mathrm{N}_{\mathrm{OC}}=225\right) \\
\mathrm{C}\left(\mathrm{N}_{\mathrm{GC}}=341,\right. \\
\left.\mathrm{N}_{\mathrm{OC}}=226\right)\end{array}$ & 890 & 0.101 & $1415^{* *}$ & $\mathbf{0 . 9 5}$ & $\mathbf{0 . 0 5}$ & {$[0.04,0.05]$} & $\mathbf{0 . 0 7}$ \\
& 0.101 & $1350^{* *}$ & $\mathbf{0 . 9 5}$ & $\mathbf{0 . 0 5}$ & {$[0.04,0.05]$} & $\mathbf{0 . 0 7}$ \\
\hline $\begin{array}{r}{ }^{a} \mathrm{GC}=\text { General Chemistry, OC }=\text { Organic Chemistry. } \\
\Delta \chi^{2} \text { between configural and metric invariant models. }\end{array}$
\end{tabular}

\section{Interpretation of Alternative SEMs}

The alternative SEMs under metric invariance conditions are displayed in Figures 5.5 and 5.6. Regression coefficients $(\beta)$ and correlations (r) are reported in their standardized form. Standardized values represent a one standard deviation effect on the independent variable for every standard deviation change in the independent variable. For 
example, in Figure $5, \beta_{\text {timel }}=0.40$ for the direct effect of verbal persuasion on the identity indicator in general chemistry. This means that for every standard deviation change in verbal persuasion, the identity indicator will increase by 0.40 of a standard deviation. Regression Pathways and Coefficients

According to the alternative model, mastery experiences influenced identity indirectly through verbal persuasion and feeling-related interest. This indirect effect was supported by a nonsignificant direct effect of mastery experiences on identity for all wording and course types (noted by the dotted arrows in Figures 5.5 and 5.6).

Within the science wording (Figure 5.5), mastery experiences had a larger direct effect on verbal persuasion, $\beta_{\text {time } 1}=0.72$ and 0.68 , compared to its direct effect on feeling-related initial interest, $\beta_{\text {time } 1}=0.33$ and 0.50 , for general and organic courses, respectively. This was also true at time 2 , with the direct effect of mastery experiences on verbal persuasion, $\beta_{\text {time } 2}=0.50$ and 0.41 , compared to its direct effect on maintained feeling-related interest, $\beta_{\text {time } 2}=0.30$ and NS, for within general and organic courses, respectively. At time 1, verbal persuasion had a similar direct effect on science identity, $\beta_{\text {time } 1}=0.40$ and 0.29 , when compared to the direct effect of initial feeling-related interest on science identity, $\beta_{\text {time } 1}=0.38$ and 0.33 , for both general and organic chemistry, respectively. In contrast, at time 2, the direct effect of verbal persuasion on science identity, $\beta_{\text {time } 2}=0.25$ and 0.38 , was larger than the direct effect of maintained feeling-related interest on science identity at time $2, \beta_{\text {time } 2}=0.18$ and 0.19 , for general and organic chemistry, respectively. The correlations between verbal persuasion and initial feeling-related interest were nonsignificant in organic chemistry and $r=0.35$ for general chemistry. In contrast, at time 2 , the correlations between verbal persuasion and 
maintained feeling-related interest were smaller at $r=0.22$ for general and became significant at $r=0.24$ for organic chemistry

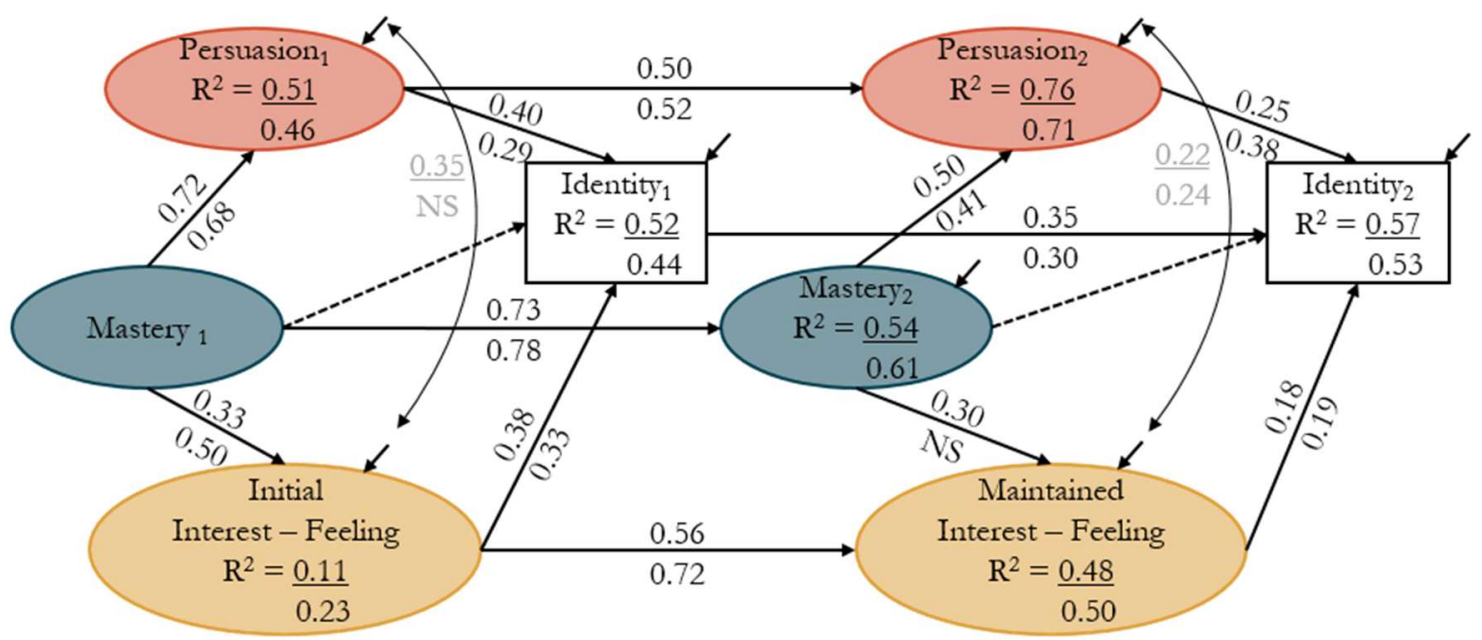

Figure 5.5. Metric invariant alternative SEMs for science worded identity constructs for general and organic chemistry. Standardized parameters for general chemistry are reported as the top values while the standardized parameters for organic chemistry are reported as the bottom values. Standardized regression coefficients are presented in black while standardized correlation coefficients are in gray. Nonsignificant pathways $(\mathrm{p}>0.05)$ are denoted with NS or by dotted arrows for nonsignificant pathways present in both courses.

The SEMs for the chemistry version (Figure 5.6) followed similar trends as the science worded version (Figure 5.5). At time 1, the direct effect of mastery experiences on verbal persuasion, $\beta_{\text {time } 1}=0.74$ and 0.69 , was larger than the direct effect of mastery experiences on initial feeling-related interest, $\beta_{\text {time } 1}=0.37$ and 0.51 , for general and organic chemistry, respectively. Again, this was true at time 2 for the direct effect of mastery experiences on verbal persuasion, $\beta_{\text {time } 2}=0.45$ and 0.45 , and the direct effect of mastery experiences on maintained feeling-related interest, $\beta_{\text {time } 2}=0.35$ and NS, for general and organic chemistry, respectively. Mirroring the trend in the science wording, the direct effect of mastery experiences on maintained feeling-related interest was not significant for organic chemistry. Different from the science worded version, the direct 
effect of verbal persuasion on chemistry identity, $\beta_{\text {time } 1}=0.24$ and 0.26 , was smaller than the direct effect of initial feeling-related interest, $\beta_{\text {time } 1}=0.53$ and 0.58 , at time 1 for both general and organic chemistry, respectively. The direct effect of verbal persuasion on chemistry identity at time $2, \beta_{\text {time } 2}=0.27$, was also smaller than the effect of maintained feeling-related interest, $\beta_{\text {time } 2}=0.38$, for general chemistry while the direct effect of verbal persuasion on chemistry identity at time $2, \beta_{\text {time } 2}=0.26$, was very similar to the effect of maintained feeling-related interest, $\beta_{\text {time } 2}=0.28$, for organic chemistry. The correlations between verbal persuasion and initial feeling-related interest were $r=0.32$ for general and nonsignificant for organic chemistry at time 1 . At time 2 , the correlations between verbal persuasion and maintained feeling-related interest were $r=0.31$ for general and became significant, $r=0.46$, for organic chemistry.

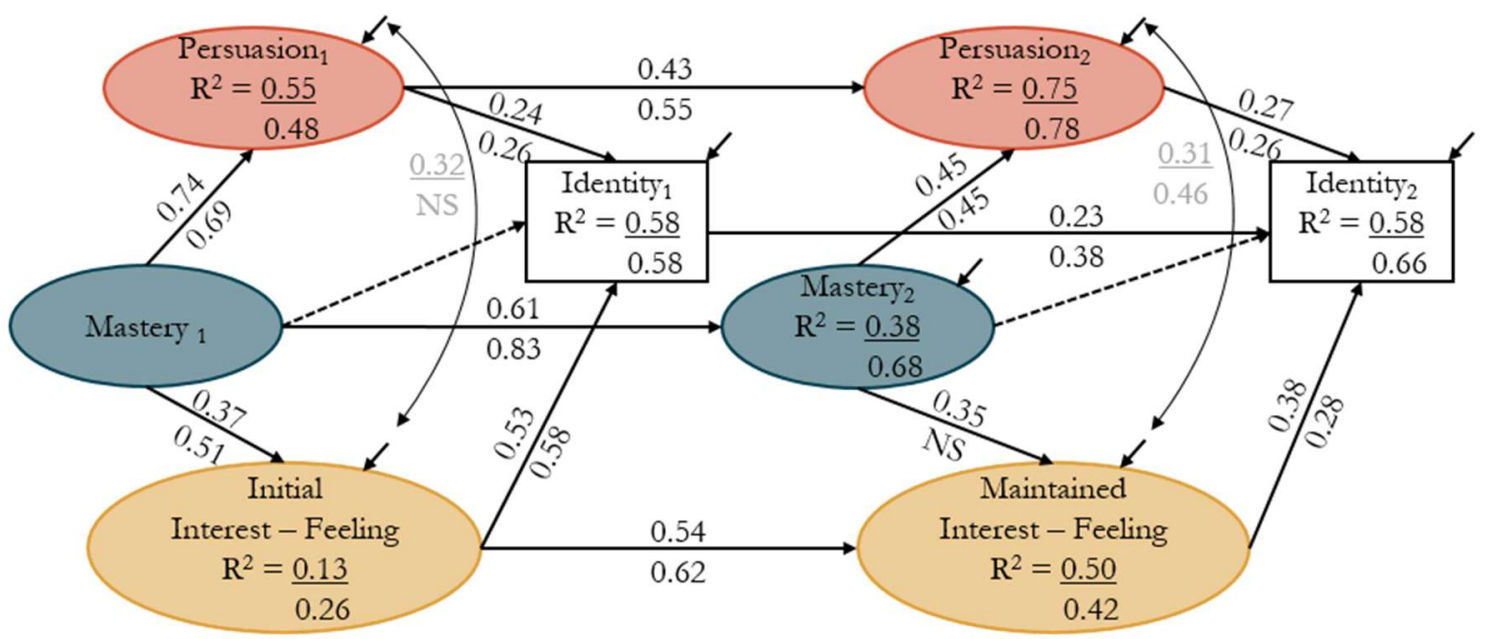

Figure 5.6. Metric invariant alternative SEMs for chemistry worded identity constructs for general and organic chemistry. Standardized parameters for general chemistry are reported as the top values while the standardized parameters for organic chemistry are reported as the bottom values. Standardized regression coefficients are presented in black while standardized correlation coefficients are in gray. Nonsignificant pathways $(p>0.05)$ are denoted with NS or by dotted arrows for nonsignificant pathways present in both courses. 
The larger direct effect of mastery experiences on verbal persuasion, as compared to both initial and maintained feeling-related interest, for both course and wording types suggested that when a student does well on mastery experiences, they are more likely to be recognized for their success rather than have their feeling-related interest stimulated. For organic chemistry, mastery experiences had a nonsignificant effect on maintained feeling-related interest for both wording types, suggesting that students' maintained feeling-related interest was not affected by success of mastery experiences. The trend in direct effects of verbal persuasion and feeling-related interest on science identity varied for both wording and course types. Within the science wording at time 1, verbal persuasion had a similar direct effect on science identity as compared to the direct effect of initial feeling-related interest on science identity for both courses. However, at time 2, verbal persuasion had a larger direct effect on science identity as compared to the direct effect of maintained feeling-related interest on science identity for both courses. Within the chemistry wording, the direct effect of verbal persuasion on chemistry identity was smaller than the direct effect of initial feeling-related interest for both courses. At time 2, in general chemistry, verbal persuasion had a smaller direct effect on identity compared to the direct effect of maintained feeling-related interest on identity while in organic chemistry, the direct effects of verbal persuasion and maintained feeling-related interest on chemistry identity were similar.

The autoregression pathways between repeated measures of mastery experiences, verbal persuasion, and identity displayed a positive predictive relation for both wording and course types. These indicated that, on average, students scored higher on the 5-point 
Likert-scale on all measures at time 2. Similarly, maintained feeling-related interest was positively predicted by initial feeling-related interest. This suggested that, on average, the initial feeling-related interest of students was stable or increased by the end of the term. Although the autoregressive pathways suggested an increase in each construct, the direct effect of each construct on identity varied over time for both wording and course type. For the science wording, the direct effect of verbal persuasion on science identity at time 1 was $\beta_{\text {time } 1}=0.40$ and 0.29 for general and organic chemistry. This same direct effect at time 2 decreased for general chemistry, $\beta_{\text {time } 2}=0.25$, and increased for organic chemistry, $\beta_{\text {time } 2}=0.38$. For the chemistry wording, the direct effect of verbal persuasion on chemistry identity at time $1, \beta_{\text {time } 1}=0.24$ and 0.26 for general and organic cheimstry, stayed stable at time $2, \beta_{\text {time } 2}=0.27$ and 0.26 . These results suggested that while students are likely to respond more positively at time 2 , this did not directly reflect the impact of the sub-constructs on identity at time 2 compared to time 1 . The same observation was made for the impact of feeling-related interest constructs on identity over time. While feeling-related interest increased between times 1 and 2, the impact of maintained feeling-related interest on identity was smaller than the impact of initial feeling-related interest on identity for all wording and course types.

\section{Identity Variance Explained}

The relations among constructs explained a considerable amount of variance within the time 1 and time 2 identity indicators. Within the science wording at time 1 , $52 \%$ and $44 \%$ of variance was explained within general and organic chemistry, respectively as compared to $57 \%$ and $53 \%$ at time 2 (Figure 5.5 ). Within the chemistry wording at time $1,58 \%$ of variance was explained within both general and organic 
chemistry as compared to $58 \%$ and $66 \%$ at time 2 (Figure 5.6). While a considerable amount of variance within the identity indicators was accounted for, the amount of unexplained variance suggests that there are other constructs involved in identity (as detected by the identity indicator) that are not being captured with these measures. This was expected, as not all of the sub-constructs proposed to be a part of science or chemistry identity were measured during this study.

\section{Conclusions}

To address research question one, the constructs of feeling- and value-related initial and maintained interest, verbal persuasion, and mastery experiences were measured and their psychometric functionality was explored in general and organic chemistry with both science and chemistry wording at two time points. As a result of issues with the initial value-related scale found through confirmatory factor analysis (CFA), the value-related interest scales were removed from further analysis. One-, two-, and three-factor CFA models were tested with both course and wording types for both time points. Global model fit indices provided evidence for acceptable model fit for all but one CFA model, the three-factor CFA with the science wording in organic chemistry. The one-factor model results supported the use of omega as an estimator for the singleadministration reliability of each scale. The two- and three-factor results supported measurement models that could be further utilized within structural equation modeling (SEM) to explore the relations between the constructs and how they relate to an identity indicator, in order to address research question two.

Prior to testing the full SEMs, longitudinal configural and metric measurement invariance was evaluated between the repeated measures of mastery experiences and 
verbal persuasion and resulted in adequate fit. Then, baseline and alternative models were tested through SEM with both course and wording types across both time 1 and time 2 measures (Figure 5.4). Although both models provided adequate fit of the data for all course and wording types, the alternative model was chosen as the preferred model based on previous results (Cribbs et al., 2015; Godwin et al., 2016) as well as support from social-cognitive theory (SCT), situational interest (Renninger \& Hidi, 2011), and science identity theory (Carlone \& Johnson, 2007). Providing evidence that similar relations existed between mastery experiences, verbal persuasion, and situational interest through the alternative model in Figure 5.4 provided quantitative support for the alignment between these constructs.

After deciding on the most appropriate model and testing multi-group invariance between general and organic chemistry, the parameters within the alternative SEM model were interpreted. A key finding within the alternative SEM model was the relation between mastery experiences and the identity indicators. Mastery experiences was found to have an indirect effect on identity, through verbal persuasion and feeling-related interest. These indirect paths provided evidence that success within the classroom alone may not influence identity formation. Providing positive feedback and facilitating interest after students perform a task successfully may be more meaningful to identity formation. The only exception to the indirect effect was the nonsignificant path between mastery experiences and maintained feeling-related interest for both wording types in organic chemistry. Although maintained feeling-related interest still positively predicted identity, 
this was not preceded by mastery experiences. This suggested that in these conditions, student success does not influence their maintained feeling-related interest.

The direct effect of verbal persuasion on identity varied between wording and course types. For the science wording, the direct effect of verbal persuasion on science identity at time 2 decreased for general chemistry, and increased for organic chemistry as compared to their effects at time 1 . At time 1 in general chemistry, verbal persuasion may be reflective of students' pre-college experiences, where time 2 may be reflective of their first experiences within college. However, when students enter organic chemistry, the measure of time 1 verbal persuasion could be more reflective of college experiences and therefore, the change over time may be more reflective of artifacts encountered in college. Another explanation of the varying strengths in the relation between verbal persuasion and identity could be that students enrolled in organic chemistry have previously been successful in general chemistry and may be more likely to have stronger connections between verbal persuasion and identity at the end of the course. For the chemistry wording, the direct effect of verbal persuasion on chemistry identity remained stable between times 1 and 2 for both course types. This suggested that chemistry identity was equally influenced by positive verbal feedback over time in both courses.

Initial feeling-related interest significantly and positively predicted identity for both course and wording types, suggesting that students' incoming feeling-related interest of science or chemistry courses was reflective of their incoming identity. Additionally, maintained feeling-related interest significantly and positively predicted identity within both course and wording type, but to a lesser extent than the initial measure. This could 
be due to misalignment between students' initial interest and course expectations as the course progresses, thereby potentially explaining the decrease in the predictability of identity through maintained feeling-related interest.

While we can compare trends in the model parameters between wording types, it would not be appropriate to compare the magnitude of the parameters. The chemistryand science-wording data had slightly different SEM model specifications due to the item error correlation with science wording of the initial feeling-related scales. Due to this difference between models, multi-group invariance between wording types could not be tested. Multi-group invariance is necessary to interpret the magnitude of parameters between groups (Fischer \& Karl, 2019).

The alternative SEM model (Figure 5.4) described the relations between verbal persuasion, mastery experiences, feeling-related interest, and an identity indicator at two time points. This model has theoretical support through SCT (Bandura \& National Inst of Mental Health, 1986), situational interest (Hidi \& Renninger, 2006), and science identity theory (Carlone \& Johnson, 2007), and the adequate fit of the data to this model supported the hypothesized relations. The alternative SEM model supported that a person performs tasks that illustrate their competence in a way that an individual is recognized by others as a credible science person (Carlone \& Johnson, 2007). It also supported that interest can be facilitated through success in mastery experiences and knowledge acquisition, as noted in SCT (Bandura \& National Inst of Mental Health, 1986) and situational interest theory (Hidi \& Renninger, 2006). As there is no explicit directional relation theorized between verbal persuasion and interest, the correlation between the two 
constructs was supported. Carlone has stated, “Of course, any boundary-defining attempts leave out other important, equally valid and rigorous, ways to bound the concept. The important thing is to understand the ways one bounds the concept and what is visible and veiled as a result(Carlone, 2012, p. 9)." The constructs of the physics identity framework (Figure 5.1B) have been qualitatively shown to align with the constructs of mindset, situational interest, verbal persuasion, vicarious experiences, and mastery experiences (Figure 5.1C) (Hosbein \& Barbera, 2020). For this study, the constructs of mastery experiences, verbal persuasion, and situational interest were chosen to mimic previous studies (Cheng et al., 2018; Cribbs et al., 2015; Godwin, Potvin, Hazari, et al., 2013; Godwin et al., 2016) in order to explore their alignment quantitatively. We recognize that the included measures and their relations are not the only constructs involved in identity formation, and that that others, such as vicarious experiences and mindset may help to gain a more holistic view.

There were multiple issues with the science wording in organic chemistry throughout analysis. Under these conditions, the three-factor models of verbal persuasion, mastery experiences, and initial-interest did not show evidence of adequate fit. The alternative model under the same conditions (Figure 5.5) provided adequate fit through joint criteria but was on the cusp of misfit. In addition, the science wording in the initialand maintained-interest scales showed evidence of the presence of two separate constructs, potentially due to the differences in responses with items framed in "this class" versus "science". Given these issues, we do not recommend using the scienceworded version of the identity measure until modifications and further studies can be 
conducted. That is, the items on the interest scales need to be re-contextualized to the classroom and the instrument re-tested. The chemistry worded version, here on referred to as the Measure of Chemistry Identity (MoChI), did not show issues with model fit and could be used in further studies to explore relations between the sub-constructs of identity within different learning environments. In addition to showing possible relations between verbal persuasion, mastery experiences, and situational interest, the alternative models in Figures 5.5 and 5.6 provided information about identity formation over time. The MoChI could therefore be used in conjunction with identity interventions to measure the change in the sub-constructs of identity and how their relations change in magnitude over time in different classroom environments.

\section{Limitations}

It is important to emphasize that validity and reliability are not properties of an instrument itself. These are properties of the data produced by an instrument (Arjoon et al., 2013; Komperda, Pentecost, et al., 2018). The data produced by the MoChI has been shown to have evidence of validity and reliability. It is of note that the models utilized in this study were modified post-hoc. Post-hoc model modifications can be data driven and therefore, models should be verified using other samples. To expand the justification for and generalizability of the MoChI and alternative model, cross-validation studies with similar and different populations are warranted (MacCallum, Roznowski, \& Necowitz, 1992). The students who participated in this study saw both science and chemistry wording on their survey, allowing them to directly compare their responses. To provide further evidence for the functioning of these measures, future distributions should separate the wording types upon administration and re-evaluate the models. 
One item on the initial value-related interest scale was not performing well psychometrically with the science wording. The consequence of this was removal of both the initial and maintained value-related interest scales. It was hypothesized that the reason behind the poor performance of the item was because some items on the scale referred to "science" while others referred to "this class". Students may not explicitly reference "this class" when responding to an item that contained "science" in it and therefore two unique constructs may have existed within the single scale. This should be explored further and the value-related interest scales edited to only reflect the class of interest.

Within this study, identity was conceptualized in a simplistic way, mimicking previous studies (Cheng et al., 2018; Cribbs et al., 2015; Godwin et al., 2016). Identity as a construct is complex and one item may not fully capture a student's science or chemistry identity. Further research is required to explore the degree to which identity can be represented by a single measure with multiple indicator variables or if this single indicator captures enough of the identity construct.

Finally, SEM does not confirm a "true" model (Mueller \& Hancock, 2008). This was evident by the adequate fit of both SEMs (Figure 5.4) with the sample data in this study. Therefore, the alternative SEM model should be interpreted as one possible explanation for the relations between identity sub-constructs. Further support of these relations should be provided through interviews that are designed to target the directional relations between constructs. 


\section{Chapter 6}

\section{Conclusions, Implications, Limitations and Future Research}

\section{Conclusions}

Fostering student identity within science disciplines has been theorized to increase student persistence toward STEM undergraduate degrees (Estrada et al., 2011; Flowers \& Banda, 2016; Graham et al., 2013). In order to evaluate the effects of identity on persistence, measures of identity that have been shown to provide valid and reliable data must be developed. While there are identity measures that currently exist (Chemers et al., 2011; Cribbs et al., 2015; Estrada et al., 2011; Hazari et al., 2010; Stets et al., 2017; Verdín et al., 2018; Vincent-Ruz \& Schunn, 2018), they are not operationalized to the “"type of person' in a given context" definition of identity (Gee, 2000) or are not operationalized to chemistry.

The research within this study was divided into two phases and provided the initial steps toward establishing more theoretically grounded measures of science and chemistry identities. The first phase consisted of a qualitative investigation to explore the alignment of the physics identity framework (Hazari et al., 2010) to science and chemistry identities and subsequently ground the identity themes in theoretically supported construct measures within the literature. The second phase of the study employed quantitative methods to further investigate alignment between the physics identity framework and the identified constructs. This final chapter discusses the conclusions found through these phases by summarizing the four research questions proposed in Chapter 1. 


\section{Phase One}

To meaningfully measure constructs involved in the formation of a science and/or chemistry identity, the constructs and their measures must be theoretically grounded. The physics identity framework previously described the constructs of performance/competence, recognition, and interest as being important in identity formation. While the constructs were defined within the established framework of science identity (Carlone \& Johnson, 2007), the alignment of items reflecting each construct remains unclear. Therefore, to further investigate the constructs, semi-structured interviews were performed with students in three chemistry courses using the physics identity framework items reframed as questions and contextualized to science and chemistry.

Q1: What themes arise when students are asked questions reflecting performance/competence, recognition, and interest, pertaining to science or chemistry, as described by the physics identity framework?

Four themes arose within the semi-structured interviews through thematic analysis (Table 4.5). Within the first theme, "interest in science or chemistry is based on feelings or values and occurs in stages", students described their interest in science or chemistry based on their feelings or values such as using science or chemistry to attain career goals. This theme overlapped with the construct of interest as outlined by the physics identity framework. The second theme, "educational experiences contribute to science or chemistry identity", contained student descriptions of participating in science using examples from educational experiences, such as past performance on exams. This theme overlapped with the performance/competence construct as described by the physics identity framework. The third theme uncovered throughout the interviews, 
"students gain information about identity through interactions with others", involved students describing their relation to science or chemistry identity through their interaction with others, such as receiving encouragement from their parents. This theme overlapped with the construct of recognition as described by the physics identity framework. The fourth and final theme, "participation in science or chemistry takes a certain kind of person", involved students describing participation in science or chemistry as requiring certain traits, such as someone who has an "intuition" for science or chemistry. This theme did not directly overlap with any construct described by the physics identity framework and was therefore a novel theme. The four themes were then compared to theoretically supported constructs described in the literature in order to further ground them within their appropriate theoretical frameworks.

Q2: To what extent do reported themes align with proposed and other affective constructs?

The four themes outlined by the first research question aligned with several theoretically supported constructs. The purpose of this alignment was to build upon the physics identity framework by grounding the constructs described by the framework (performance/competence, recognition, and interest) within more established theoretical frameworks. Defining and grounding these constructs within established theoretical frameworks supported the content validity for operationalization to science and chemistry.

The first theme stated that student interest is related to value or feelings and occurs in stages aligned with feeling- and value- related interest as described by Schiefele (1991) as well as the four-phases of interest model (Hidi \& Renninger, 2006). Schiefele 
(1991) has proposed that there are two components to interest: value-related and feelingrelated. Value-related interest occurs because of the value one places on a particular subject. For example, an individual being interested in chemistry because of its importance in solving real-world problems. Feeling-related interest occurs because of positive feelings one has toward a particular subject such as enjoyment one experiences when participating in chemistry. The four-phase model of interest (Hidi \& Renninger, 2006) consists of triggering situational interest, maintained situational interest, emerging individual interest, and well-developed individual interest. The two situational phases are early phases of interest, where the environment still has an effect on one's interest. Interest then moves to the two individual phases, where interest is more internalized and long-lasting. In the context of identity development as a student, situational interest more closely aligns with interest as described by the physics identity framework. This is due to the fact that situational interest is influenced by environmental factors, rather than selfgenerated, and therefore more meaningful to measure for the future goal of studying interventions that could influence identity formation.

The second theme consisted of students describing their educational experiences in reference to their science or chemistry identity. This theme aligned with mastery experiences, a source of self-efficacy described by Social Cognitive Theory (SCT) (Bandura \& National Inst of Mental Health, 1986). Mastery experiences are those in which an individual can base their self-perception of future tasks on successes and failures of specific tasks. In a science or chemistry identity context, a mastery experience could be taking an exam. Students frequently mentioned passing exams or courses when 
discussing their science or chemistry identity. Of note, this theme aligned with Carlone and Johnson's original definitions of performance and competence (Carlone \& Johnson, 2007) rather than the definitions outlined by the physics identity framework (Hazari et al., 2010) because students were directly describing their competency and performance in their education pertaining to science or chemistry when discussing identity. They did not explicitly mention confidence when unprompted, but instead described their mastery experiences, such as taking an exam.

The third theme involved students describing their science or chemistry identity based on interactions with others. There were three distinct ways that students described these interactions: through comparison of themselves to others, being approached for help with or discussing science or chemistry, and encouragement or discouragement from others. Two of these three interaction types aligned with additional sources of selfefficacy, vicarious experiences and verbal persuasion. Vicarious experiences are experiences in which an individual bases their self-perception of completing a specific task on the observation of another individual or individuals performing the task (Bandura \& National Inst of Mental Health, 1986). Students compared themselves to classmates or others in their lives when discussing their own science or chemistry identities. Verbal persuasion is the notion that self-perception of success in a task is based on encouragement or discouragement from others (Bandura \& National Inst of Mental Health, 1986). Several students noted being encouraged or discouraged by people in their lives about participation in science or chemistry. 
The fourth theme entailed students describing participation in science or chemistry as taking a certain "type of person". This theme aligned with the construct of mindset, where one has either a growth or fixed mindset (Dweck, 2013). A growth mindset indicates that a person believes one has the ability to develop their intelligence, whereas a fixed mindset indicates that a person believes intelligence is innate and cannot be changed. Some students described science or chemistry people as committed and driven to find answers which suggested more of a growth mindset while others described them as having an inherent trait which indicated a fixed mindset. This theme and the construct of mindset was not a part of the physics identity framework or science identity theory (Carlone \& Johnson, 2007), making it a novel construct proposed to be involved in identity formation.

Through the semi-structured interviews performed within phase one, the physics identity framework constructs of recognition, performance/competence, and interest were further investigated and shown to align with five theoretically supported constructs: situational interest, mastery experiences, vicarious experiences, verbal persuasion, and mindset. Four of the theoretically supported constructs overlapped with the physics identity constructs (situational interest with interest, verbal persuasion and vicarious experiences with recognition, and mastery experiences with performance/competence) while the construct of mindset was novel. Providing qualitative evidence for the overlap between the physics identity framework with theoretically supported constructs provided the first step for developing more grounded science and chemistry identity measures. 


\section{Phase Two}

The conclusions drawn from phase one of the study provided a starting point to measure more theoretically grounded constructs involved in science or chemistry identity. While the qualitative study found alignment between the categories of the physics identity framework and theoretically supported constructs from the literature, the questions within phase two take the investigation a step further by exploring the alignment quantitatively. Therefore, moving forward, only those constructs that directly overlapped with the physics identity framework were used in the quantitative analysis. This decision was made in order to mimic previous analyses for the purpose of providing additional evidence of the overlap of interest with situational interest, recognition with verbal persuasion, and performance/competence with mastery experiences. While the construct of recognition showed overlap with both vicarious experiences and verbal persuasion, verbal persuasion was chosen over vicarious experiences as it is a more direct form of recognition and therefore was more appropriately aligned with how recognition was initially defined in the physics identity framework.

Q3: To what degree will an instrument containing items designed to measure mastery experiences, verbal persuasion, and situational interest show psychometric functionality in undergraduate chemistry courses with science-specific wording or chemistry-specific wording?

Previously existing scales developed to measure feeling- and value-related initial and maintained situational interest (Ferrell \& Barbera, 2015), mastery experiences (Usher \& Pajares, 2009), and verbal persuasion (Usher \& Pajares, 2009) were modified to fit a college setting, contextualized to reflect science and chemistry identity wording, and distributed to general and organic chemistry students. Students saw both wording types in a single survey in order to compare science and chemistry wording responses. The survey 
was distributed at the beginning and end of the courses and the psychometric functioning of the scales was then investigated. One-, two- and multi-factor confirmatory factor analysis (CFA) models were tested. One-factor models were tested for the purpose of justifying reliability the estimates. Two-factor models were tested in order to mimic previous literature analyses (Ferrell \& Barbera, 2015; Usher \& Pajares, 2009). Multifactor models were tested to provide evidence of measurement model functioning before studying the relations between constructs using structural equation modeling (SEM).

Two-factor CFA models consisting of feeling- with value-related interest (for both the initial and maintained scales), and mastery experiences with verbal persuasion at times 1 and 2 were first tested to mimic the way that the scales have been previously tested within the literature (Ferrell \& Barbera, 2015; Usher \& Pajares, 2009). This was done to ensure that the minor changes in wording did not impact the psychometric functioning of the scales. Modification indices on the two-factor initial interest CFA models revealed item error correlations within the science worded version of the scales. Four of the seven items contained the phrase "this class", for example, "I chose to take this class because I'm really interested in the topic." The remaining three items either contained the word "chemistry" or "science", for example, "I am really looking forward to learning more about [science]." Items containing "this class" were correlated with each other as compared to words containing the word "science". After item error correlations were accounted for within the models, all two-factor feeling- and value- related interest models provided adequate fit for both wording and course types (Table 5.1). Even though the models met globally fit parameters, there was one item on the science worded initial 
value-related interest scale that caused alarm with low factor loadings of 0.41 and 0.27 in general and organic chemistry courses, respectively. In addition to the low loadings, the item had high mean, skew, and kurtosis across both wording and course types. This item was removed from the initial value-related scale. However, after discarding this item, there remained only two items on the science worded initial value-related scale, which created an issue for further analysis because at least three items are required per scale for factor analysis (Kline, 2016). Consequently, all value-related interest scales were removed from further analysis. One-factor CFA models of initial and maintained feelingrelated interest were tested to ensure that the feeling-related interest scales functioned as intended without the correlated value-related interest scales. All feeling-related interest models provided adequate fit for both wording and course types (Table B.4). The modification indices from the two-factor CFA models of mastery experiences and verbal persuasion revealed item error correlations between two items on the mastery experiences scale that reference past successes vs present. After this modification, two-factor models of mastery experiences and verbal persuasion had adequate model fit at both time 1 and time 2 for both wording and course types (Table 5.2).

The scales that showed adequate psychometric functioning were combined to form three-factor CFA models at times 1 and 2 for both wording and course types. This step was taken in order to show adequate measurement functioning when all scales are combined before moving to SEM to study the relations between constructs. Time 1 CFA models, consisting of initial feeling-related interest, mastery experiences, and verbal persuasion, provided evidence of adequate fit for both wording and course types except 
for the science wording in organic chemistry (Table 5.3). The fit was on the cusp of adequate but was retained for further analysis. All three-factor CFA models at time 2, consisting of maintained feeling-related interest, mastery experiences, and verbal persuasion, provided evidence of adequate fit for both wording and course types (Table 5.3). Confirming the fit of two- and three-factor CFA models provided evidence of structural validity for the scales used in this study.

Single-administration reliability estimates assume unidimensionality of a measure. Therefore, one-factor congeneric CFA models were tested to provide structural validity evidence of unidimensionality for each individual construct across both time points and with both wording and course types (Table B.4). All one-factor congeneric CFA models provided evidence of adequate fit. Reliability was therefore reported for each scale in the form of omega. All omega estimates were above the recommended cutoff of 0.7 (Hancock \& Freeman, 2001) for both wording and course types at each time point (Table 5.4). The ranges of omega provided evidence that the items measuring each individual construct (the true construct variance) explained $74-92 \%$ of the observed variance.

All CFA models provided evidence of adequate fit except for the three-factor CFA at time 1 with the science wording in organic chemistry. Omega values of each individual scale fell within the suggested cutoff criteria, providing evidence of reliability. Providing evidence of structural validity and reliability of these scales allowed us to move forward in studying the relations between constructs involved in identity formation. 
Q4: What are the relations between mastery experiences, verbal persuasion, situational interest and a science identity indicator or a chemistry identity indicator?

To answer the final research question, SEM was utilized to study the relations between mastery experiences, verbal persuasion, situational interest, and a science or chemistry identity indicator. Models from the physics identity framework (Figure 5.2) were modified to include the constructs at two time points (Figure 5.4). These SEMs were used to model data from both the science- and chemistry-worded surveys within general and organic chemistry. Before full SEMs were run, metric invariance was established between the repeated measures of mastery experiences and verbal persuasion at times 1 and 2. Next, both baseline and alternative models from Figure 5.4 were tested and showed equal and adequate fit with both wording and course types. The alternative model was chosen as the preferred model based on previous results (Cribbs et al., 2015; Godwin et al., 2016) as well as support from SCT (Bandura \& National Inst of Mental Health, 1986), the theory of situational interest (Hidi \& Renninger, 2006), and science identity theory (Carlone \& Johnson, 2007).

After choosing to move forward with the alternative model, metric invariance was established between models from general and organic chemistry within the same wording type in order to interpret model parameters (Figures 5.5 and 5.6). The key finding within the alternative model was the relation of mastery experiences and the identity indicator for both wording and course types. Mastery experiences was indirectly related to the identity indicator through feeling-related interest and verbal persuasion. Therefore, providing positive feedback and facilitating interest after students perform a task successfully may be more meaningful to identity formation rather than success on a task 
alone. The only exception to the indirect effect between mastery experiences and identity through feeling-related interest and verbal persuasion was the nonsignificant path between mastery experiences and maintained feeling-related interest for both wording types in organic chemistry. Although maintained feeling-related interest still positively predicted identity, this was not preceded by mastery experiences. This suggested that in organic chemistry, student success does not influence their feeling-related interest at the end of a course. The alternative model outcomes, with data from the newly defined constructs, match the prior results from the literature (Cribbs et al., 2015; Godwin et al., 2016). This provided quantitative support for the alignment between the newly defined constructs and those from the original physics identity framework.

While both alternative SEMs provided evidence of adequate fit under metric invariance, there were multiple issues with the science wording in organic chemistry throughout analysis. Under these conditions, the three-factor CFA model of initial feeling-related interest, mastery experiences, and verbal persuasion did not provide evidence of adequate fit. In addition, the alternative model SEM under the same conditions, was on the cusp of misfit. Finally, the initial and maintained feeling-related scales showed evidence of containing more than one construct due to differences in student responses to items containing "this class" versus "science". The way that students define "science" within the context of the survey may vary between students and needs to be investigated, as this could be a reason for the measurement issues within the science worded-survey. Due to these complications, we do not recommend the use of the science worded version of the identity measure until further modifications and analysis can be 
completed. The chemistry worded identity measure, the Measure of Chemistry Identity (MoChI), did not share similar complications and could be used in further studies to investigate differences in the constructs of identity within different environments and how these constructs change over time.

\section{Limitations and Future Research}

Within the first phase of this research, science and chemistry identities were not investigated separately. While no novel themes arose when students discussed science versus chemistry in relation to identity, there was evidence of the distinction between the two wording types within the second phase of the study. Within cognitive interviews, students responded differently to items on the initial and maintained feeling-related scales that contained "science" versus "this class" while responding similarly to items containing "chemistry" versus "this class". This provided evidence that students may be citing a variety of experiences outside of their class when asked about "science". Throughout the survey, many students discussed "chemistry" in a way that only related to undergraduate courses, providing evidence that student experiences with chemistry outside of class was limited. The courses included within this study were chemistry courses taken early in students' undergraduate career. The way students use the term "chemistry" could change over time to include experiences outside of the classroom, such as undergraduate research in STEM. It is important to note that based on the selfreported demographics, a majority of students were non-chemistry majors. This implied that non-chemistry majors see themselves as "chemistry people" and further exploration of why would be insightful. Nuances between students' definitions of science and 
chemistry identities should be further evaluated after separate scale administrations and in more advanced chemistry courses.

Identity was measured using one indicator item, I see myself as a [science] person, where the bracketed portion was replaced with "chemistry" for the chemistryworded version. This was done in order to mimic previous analyses with the physics identity framework. The item originates from the overarching definition of identity as presented by Gee (2000). In his seminal paper, Gee breaks down identity further into N-, I-, D-, and A-identities, as discussed in Chapter 1. Creating measures that reflect each of these identities and using them instead of the single identity item could provide even more information about identity formation. For example, A-identities are comprised of experiences within an "affinity group". A cohort of students within a science classroom could be seen as an affinity group as students within the course may share nothing else in common except for the experiences they share within the classroom. If the relations between the sub-constructs of identity and an A-identity operationalized to the science classroom were modeled, would we see different patterns of relations emerging? Having more information about identity than a single identity indicator would be valuable for future research studies.

There were a few issues with the science worded version of the identity measure during analysis. To investigate these issues further, the initial feeling-related scale should be modified and redistributed to reflect a single construct rather than continuing to distribute items that contain both "science" and "this class". The initial and maintained value-related interest scales were dropped from analysis due to issues with one of the 
items on the initial value-related scale. This issue should be investigated further through cognitive interviews in order to either modify the item or add other relevant value-related interest items in order to re-test this scale and study its relation to feeling-related interest, mastery experiences, and verbal persuasion.

SEM does not provide a "true" model of causation (Mueller \& Hancock, 2008). The final alternative SEM (Figure 5.5 and Figure 5.6) described the relations between situational interest, verbal persuasion, and mastery experiences in one proposed direction. This model did not account for the effects of identity at time 1 on any of the constructs at time 2 , except for the autoregressive pathway between identity at time 1 and time 2 . These relations were intentionally omitted in order to make this two time-point model as simple as possible while still mirroring models tested in previous literature using the physics identity framework (Figure 5.2B). In order to test the effects of identity on situational interest, mastery experiences, and verbal persuasion, these paths should be added to the alternative SEM and re-tested. Qualitative analysis should be carried out alongside quantitative re-testing to provide more evidence of dominating causal relations.

The DBER report (2012) has also called for additional research on multiple dimensions of the affective domain and how they impact retention, specifically for different demographic groups, such as females (p.162). Understanding how science identity is formed and sustained, with intersectionality considered, is crucial to providing learning environments that could aid in improving the retention of underrepresented populations within the STEM pipeline. The first step in studying more generalized group differences in identity is to have a robust measure that has the same psychometric 
functioning for multiple groups. Data from the MoChI was supported with evidence of reliability, multiple facets of validity, and shown to be metric invariant within the chemistry wording for both chemistry courses. This allowed for the interpretation of SEM parameter differences at the course level. In future distributions, metric invariance should be established between demographic groups, the alternative model tested, and differences in identity construct relations evaluated.

While metric invariance allows for the comparison of model parameters, it does not allow for direct comparisons of construct means between groups. Brickhouse and colleagues (2000) emphasized the need to understand how different groups of students identify with science to better create supportive learning environments. In order to evaluate the differences in means of each construct between demographics, strict invariance must be established for each individual construct so that comparisons can be made based on different learning environments in order to explore whether different teaching practices affect the identity formation in unique ways for different groups. As long as appropriate levels of invariance are established over time and between the particular groups under study, as was demonstrated in this study between two course types, the MoChI has the potential to be used as a tool to study possible differences in identity formation between different demographic groups in addition to measuring change in identity constructs over the course within different learning environments.

Carlone has stated, "Of course, any boundary-defining attempts leave out other important, equally valid and rigorous, ways to bound the concept. The important thing is to understand the ways one bounds the concept and what is visible and veiled as a result 
(Carlone, 2012).” For this study, the constructs of mastery experiences, verbal persuasion, and situational interest were chosen to mimic previous studies (Cheng et al., 2018; Cribbs et al., 2015; Godwin et al., 2016) in order to explore their alignment with the constructs of physics identity quantitatively. We recognize that the chosen measures and their relations are not the only constructs involved in identity formation, and that measuring others, such as value-related situational interest, vicarious experiences and mindset may help to gain a more holistic view.

\section{Implications for Researchers}

Both quantitative and qualitative methods were used within this study to aid in addressing an overarching issue in Discipline-Based Education Research (DBER). In 2012 , it was reported that less than $40 \%$ of students entering college with the intention to major in STEM actually pursue and obtain a STEM degree (President's Council of Advisors on Science and Technology, 2012). One of the suggestions to increase retention was to focus on changes within learning environments, such as active learning (President's Council of Advisors on Science and Technology, 2012, p. 17). One proposed mechanism by which learning environments can increase student retention is through identity (Graham et al., 2013). In order to confirm this hypothesis, measures of identity that provide valid and reliable data need to be available.

The end goal of this study was to provide the field of chemistry education with theoretically grounded measures of science and chemistry identity that have shown evidence of providing valid and reliable data. While the science identity measure provided evidence of adequate model fit for most CFA and SEM models, the three-factor CFA model with science wording in organic chemistry showed evidence of misfit and the 
alternative SEM model was on the cusp of misfit. In addition, the initial feeling-related interest scale showed evidence of the presence of two constructs through cognitive interviews with students. Therefore, until further modification and analysis can be performed with the science wording, the science identity measure is not recommended for use. The Measure of Chemistry Identity (MoChI) did not show issues with model fit and could be used in further studies to explore the relations between situational interest, mastery experiences, and verbal persuasion. Using the MoChI to obtain pre- and postsurvey information could provide insight into changes into identity across different learning environments.

\section{Implications for Practitioners}

Utilizing SEM in the classroom to study identity may not be practical for many practitioners due to barriers such as small course sizes or lack of training with the method. Despite this, there are valuable takeaways from this research that can be connected to chemistry education practice in order to facilitate positive chemistry identity formation. Through the alternative SEM, we found that mastery experiences are not directly related to chemistry identity, but there is a direct relation from both situational interest and verbal persuasion to chemistry identity. There have been several studies that provide evidence that situational interest can be affected by classroom variables such as instructors showing interest or concern for their students, (Rotgans \& Schmidt, 2011), instructors providing evidence of their knowledge of the subject (Rotgans \& Schmidt, 2011), instructors explaining concepts to students in an understandable manner (Rotgans $\&$ Schmidt, 2011), repeated exposure to practice problems designed to improve situational interest (Rotgans \& Schmidt, 2017), characteristics of lectures (Quinlan, 
2019), and student perceptions of the utility and meaningfulness of interventions (Hunsu, Adesope, \& Van Wie, 2017). Verbal persuasion can be facilitated through positive feedback to an individual from instructors or peers. Bandura (1997) offers a word of caution when it comes to verbal persuasion, stating that any verbal persuasion needs to be within realistic bounds. If an individual is persuaded with too many positive comments and subsequently fails, that persuader will be discredited and unable to effect change. As an instructor, providing realistic feedback to students after participation in a mastery experience such as homework or an exam may provide more impact on identity formation as opposed to providing blanket positive statements to all students.

Although their causal relations to identity were not studied, we provided evidence, through phase one of the study, that mindset and vicarious experiences are also involved in science and chemistry identity formation. Mindset interventions currently exist with the goal of shifting students to a growth mindset, i.e., that intelligence is malleable and not fixed and these interventions have been shown to promote incremental views of intelligence (DeBacker et al., 2018), improve effort (Sriram, 2014) and improve performance (Broda et al., 2018; Yeager \& Dweck, 2012) in students. The notion that a role model's influence on an individual is higher when the role model has similar demographic characteristics is not new (Basow \& Howe, 1980; Karunanayake \& Nauta, 2004; Lockwood, 2006). Vicarious experiences can be facilitated in a positive way by showing students examples of successful scientists from a wide variety of backgrounds (Schinske, Perkins, Snyder, \& Wyer, 2016). While the results contained in this research study do not give a completed map of the casual relations between identity for all 
undergraduate STEM students, it laid a foundation for practitioners to implement interventions that influence theoretically supported constructs involved in science and chemistry identity formation. 


\section{References}

Abrams, D., \& Hogg, M. A. (1990). Social identity theory: Constructive and critical advances. New York, NY: Springer-Verlag Publishing.

Ainley, M. (2007). Being and Feeling Interested: Transient State, Mood, and Disposition. In P. A. Schutz \& R. Pekrun (Eds.), Emotion in Education (pp. 147-163). Cambridge, MA: Academic Press.

Allen, M. J., \& Yen, W. M. (2001). Introduction to measurement theory. Long Grove, IL: Waveland Press.

American Educational Research Association, American Psychological Association, \& National Council on Measurement in Education. (2014). Standards for Educational and Psychological Testing. Washington, DC: American Educational Research Association.

Arjoon, J. A., Xu, X., \& Lewis, J. E. (2013). Understanding the State of the Art for Measurement in Chemistry Education Research: Examining the Psychometric Evidence. Journal of Chemical Education, 90(5), 536-545. doi:10.1021/ed3002013

Aschbacher, P. R., Li, E., \& Roth, E. J. (2010). Is science me? High school students' identities, participation and aspirations in science, engineering, and medicine. Journal of Research in Science Teaching, 47(5), 564-582. doi:10.1002/tea.20353

Bandura, A. (1977). Self-efficacy: Toward a unifying theory of behavioral change. Psychological Review, 84(2), 191-215. doi:10.1037/0033-295X.84.2.191

Bandura, A. (1997). Self-efficacy: The exercise of control. New York, NY, US: Worth Publishers.

Bandura, A., Barbaranelli, C., Caprara, G. V., \& Pastorelli, C. (2001). Self-efficacy beliefs as shapers of children's aspirations and career trajectories. Child Development, 72(1), 187206. doi:10.1111/1467-8624.00273

Bandura, A., \& National Inst of Mental Health. (1986). Prentice-Hall series in social learning theory: Social foundations of thought and action: A social cognitive theory. Englewood Cliffs, NJ: Prentice-Hall, Inc.

Barton, A. C., Kang, H., Tan, E., O’Neill, T. B., Bautista-Guerra, J., \& Brecklin, C. (2013). Crafting a Future in Science: Tracing Middle School Girls' Identity Work Over Time and Space. American Educational Research Journal, 50(1), 37-75. doi: $10.3102 / 0002831212458142$

Basow, S. A., \& Howe, K. G. (1980). Role-model influence: Effects of sex and sex-role attitude in college students. Psychology of Women Quarterly, 4(4), 558-572. doi:10.1111/j.14716402.1980.tb00726.x

Basu, S. J., Barton, A. C., Clairmont, N., \& Locke, D. (2009). Developing a framework for critical science agency through case study in a conceptual physics context. Cultural Studies of Science Education, 4(2), 345-371. doi:10.1007/s11422-008-9135-8

Bentler, P. M. (1990). Comparative fit indexes in structural models. Psychological Bulletin, 107(2), 238. doi:10.1037/0033-2909.107.2.238

Bloom, B. S. (1956). Taxonomy of educational objectives: The classification of educational goals: Cognitive Domain. London, England: Longman, Green Co.

Bonous-Hammarth, M. (2000). Pathways to Success: Affirming Opportunities for Science, Mathematics, and Engineering Majors. Journal of Negro Education, 69(1/2), 92-111.

Braun, V., \& Clarke, V. (2006). Using thematic analysis in psychology. Qualitative Research in Psychology, 3(2), 77-101. doi:10.1191/1478088706qp063oa

Braun, V., Clarke, V., Hayfield, N., \& Terry, G. (2019). Thematic Analysis. In P. Liamputtong (Ed.), Handbook of Research Methods in Health Social Sciences (pp. 843-860). Singapore: Springer Singapore. 
Brickhouse, N. W. (2000). Embodying science: A feminist perspective on learning. Journal of Research in Science Teaching, 38(3), 282-295. doi:10.1002/10982736(200103)38:3<282::AID-TEA1006>3.0.CO;2-0

Brickhouse, N. W., Lowery, P., \& Schultz, K. (2000). What Kind of a Girl Does Science? The Construction of School Science Identities. Journal of Research in Science Teaching, 37(5), 441-458. doi:10.1002/(SICI)1098-2736(200005)37:5<441::AID-TEA4>3.0.CO;23

Brickhouse, N. W., \& Potter, J. T. (2001). Young women's scientific identity formation in an urban context. Journal of Research in Science Teaching, 38(8), 965-980. doi:10.1002/tea.1041

Broda, M., Yun, J., Schneider, B., Yeager, D. S., Walton, G. M., \& Diemer, M. (2018). Reducing Inequality in Academic Success for Incoming College Students: A Randomized Trial of Growth Mindset and Belonging Interventions. Journal of Research on Educational Effectiveness, 11(3), 317-338. doi:10.1080/19345747.2018.1429037

Brotman, J. S., \& Moore, F. M. (2008). Girls and science: A review of four themes in the science education literature. Journal of Research in Science Teaching, 45(9), 971-1002. doi:10.1002/tea.20241

Brown, T. A. (2014). Confirmatory factor analysis for applied research. New York, NY: Guilford Publications.

Burke, P. J., \& Stets, J. E. (2009). Identity theory. New York, New York, USA: Oxford University Press.

Cai, L. (2013). Factor Analysis of Tests and Items. In K. F. Geisinger, B. A. Bracken, J. F. Carlson, J.-I. C. Hansen, N. R. Kuncel, S. P. Reise, \& M. C. Rodriguez (Eds.), APA handbook of testing and assessment in psychology, Vol. 1: Test theory and testing and assessment in industrial and organizational psychology (pp. 85-100). Washington, DC: American Psychological Association.

Carlone, H. B. (2012). Methodological Considerations for Studying Identities in School Science. In M. Varelas (Ed.), Identity Construction and Science Education Research: Learning, Teaching, and Being in Multiple Contexts (pp. 9-25). Rotterdam, Netherlands: SensePublishers.

Carlone, H. B., \& Johnson, A. (2007). Understanding the science experiences of successful women of color: Science identity as an analytic lens. Journal of Research in Science Teaching, 44(8), 1187-1218. doi:10.1002/tea.20237

Cass, C. A. P., Hazari, Z., Cribbs, J., Sadler, P. M., \& Sonnert, G. (2011, October 12-15). Examining the impact of mathematics identity on the choice of engineering careers for male and female students. Paper presented at the 2011 Frontiers in Education Conference (FIE), Rapid City, SD.

Chang, M. J., Eagan, M. K., Lin, M. H., \& Hurtado, S. (2011). Considering the Impact of Racial Stigmas and Science Identity: Persistence Among Biomedical and Behavioral Science Aspirants. The Journal of Higher Education, 82(5), 564-596. doi:10.1353/jhe.2011.0030

Chemers, M. M., Zurbriggen, E. L., Syed, M., Goza, B. K., \& Bearman, S. (2011). The role of efficacy and identity in science career commitment among underrepresented minority students. Journal of Social Issues, 67(3), 469-491. doi:10.1111/j.15404560.2011.01710.x

Cheng, H., Potvin, G., Khatri, R., Kramer, L. H., Lock, R. M., \& Hazari, Z. (2018, August 1-2, 2018). Examining physics identity development through two high school interventions. Paper presented at the Physics Education Research Conference 2018, Washington, DC. 
Cheryan, S., Master, A., \& Meltzoff, A. N. (2015). Cultural stereotypes as gatekeepers: increasing girls' interest in computer science and engineering by diversifying stereotypes. Frontiers in Psychology, 6(49). doi:10.3389/fpsyg.2015.00049

Cheung, G. W., \& Rensvold, R. B. (2002). Evaluating goodness-of-fit indexes for testing measurement invariance. Structural Equation Modeling, 9(2), 233-255. doi:10.1207/S15328007SEM0902_5

Chickering, A. W., \& Reisser, L. (1993). Education and Identity. (2nd ed.). San Fransisco, CA: Jossey-Bass Inc.

Childers, G., \& Jones, M. G. (2017). Learning from a distance: high school students' perceptions of virtual presence, motivation, and science identity during a remote microscopy investigation. International Journal of Science Education, 39(3), 257-273. doi:10.1080/09500693.2016.1278483

Claro, S., Paunesku, D., \& Dweck, C. S. (2016). Growth mindset tempers the effects of poverty on academic achievement. Proceedings of the National Academy of Sciences of the United States of America, 113(31), 8664-8668. doi:10.1073/pnas.1608207113

Cribbs, J. D., Hazari, Z., Sonnert, G., \& Sadler, P. M. (2015). Establishing an Explanatory Model for Mathematics Identity. Child Development, 86(4), 1048-1062. doi:10.1111/cdev.12363

Cronbach, L. J. (1951). Coefficient alpha and the internal structure of tests. Psychometrika, 16(3), 297-334. doi:10.1007/bf02310555

Cronbach, L. J., \& Meehl, P. E. (1955). Construct validity in psychological tests. Psychological Bulletin, 52(4), 281-302. doi:10.1037/h0040957

Curran, P. J., West, S. G., \& Finch, J. F. (1996). The robustness of test statistics to nonnormality and specification error in confirmatory factor analysis. Psychological methods, 1(1), 16. doi:10.1037/1082-989x.1.1.16

Davidoff, L., \& Hall, C. (1987). Family fortunes: Men and women of the English middle class, 1780-1850. Chicago, IL: The Universtiy of Chicago Press.

DeBacker, T. K., Heddy, B. C., Kershen, J. L., Crowson, H. M., Looney, K., \& Goldman, J. A. (2018). Effects of a one-shot growth mindset intervention on beliefs about intelligence and achievement goals. Educational Psychology, 38(6), 711-733. doi:10.1080/01443410.2018.1426833

Dolan, C. V. (1994). Factor analysis of variables with 2, 3, 5 and 7 response categories: A comparison of categorical variable estimators using simulated data. British Journal of Mathematical and Statistical Psychology, 47(2), 309-326. doi:doi.org/10.1111/j.20448317.1994.tb01039.x

Dweck, C. S. (2009). Can we make our students smarter? Education Canada, 49(4), 56-61.

Dweck, C. S. (2013). Self-theories: Their role in motivation, personality, and development. New York, NY: Psychology Press.

Dweck, C. S. (2015). Carol Dweck revisits the growth mindset. Education Week, 35(5), 20-24.

Eccles, J. S. (2007). Where Are All the Women? Gender Differences in Participation in Physical Science and Engineering Why aren't more women in science?: Top researchers debate the evidence. (pp. 199-210). Washington, DC: American Psychological Association.

Eccles, J. S. (2009). Who Am I and What Am I Going to Do With My Life? Personal and Collective Identities as Motivators of Action. Educational Psychologist, 44(2), 78-89. doi: $10.1080 / 00461520902832368$

Eccles, J. S., \& Wigfield, A. (2002). Motivational beliefs, values, and goals. Annual Review of Psychology, 53(1), 109-132. doi:10.1146/annurev.psych.53.100901.135153

Estrada, M., Burnett, M., Campbell, A. G., Campbell, P. B., Denetclaw, W. F., Gutiérrez, C. G., . . Zavala, M. (2016). Improving Underrepresented Minority Student Persistence in STEM. CBE—Life Sciences Education, 15(3), es5. doi:10.1187/cbe.16-01-0038 
Estrada, M., Hernandez, P. R., \& Schultz, P. W. (2018). A longitudinal study of how quality mentorship and research experience integrate underrepresented minorities into STEM careers. CBE Life. Sci. Educ., 17(1). doi:10.1187/cbe.17-04-0066

Estrada, M., Woodcock, A., Hernandez, P. R., \& Schultz, P. W. (2011). Toward a model of social influence that explains minority student integration into the scientific community. Journal of Educational Psychology, 103(1), 206. doi:10.1037/a0020743

Fencl, H., \& Scheel, K. (2003, August 6-7). Pedagogical approaches, contextual variables, and the development of student self-efficacy in undergraduate physics courses. Paper presented at the Physics Education Research Conference, Madison, WI.

Ferrell, B., \& Barbera, J. (2015). Analysis of students' self-efficacy, interest, and effort beliefs in general chemistry. Chemistry Education Research and Practice, 16(2), 318-337. doi:10.1039/C4RP00152D

Finney, S. J., \& DiStefano, C. (2006). Nonnormal and categorical data in structural equation modeling. In G. R. Hancock \& R. O. Mueller (Eds.), Structural equation modeling: a second course (pp. 269-314). Greenwhich, CT: Information Age Publishing.

Fischer, R., \& Karl, J. A. (2019). A Primer to (Cross-Cultural) Multi-Group Invariance Testing Possibilities in R. Frontiers in Psychology, 10. doi:10.3389/fpsyg.2019.01507

Flowers, A. M., \& Banda, R. (2016). Cultivating science identity through sources of self-efficacy. Journal for Multicultural Education, 10(3), 405-417. doi:10.1108/JME-01-2016-0014

Fortus, D. (2014). Attending to affect. Journal of Research in Science Teaching, 51(7), 821-835. doi:10.1002/tea.21155

Freeman, S., Eddy, S. L., McDonough, M., Smith, M. K., Okoroafor, N., Jordt, H., \& Wenderoth, M. P. (2014). Active learning increases student performance in science, engineering, and mathematics. Proceedings of the National Academy of Sciences, 111(23), 8410-8415. doi:10.1073/pnas.1319030111

Furr, R. M., \& Bacharach, V. R. (2008). Psychometrics: an introduction. Thousand Oaks, CA: Sage Publications.

Gee, J. P. (2000). Identity as an Analytic Lens for Research in Education. Review of Educational Research, 25, 99-125. doi:10.2307/1167322

Geisinger, K. F., Bracken, B. A., Carlson, J. F., Hansen, J.-I. C., Kuncel, N. R., Reise, S. P., \& Rodriguez, M. C. (2013). APA handbook of testing and assessment in psychology, Vol. 1: Test theory and testing and assessment in industrial and organizational psychology. Washington, DC: American Psychological Association.

Gibson, W. J., \& Brown, A. (2009). Identifying themes, codes, and hypotheses Working with qualitative data (pp. 127-144). Thousand Oaks, CA: Sage Publications.

Glynn, S. M., Brickman, P., Armstrong, N., \& Taasoobshirazi, G. (2011). Science motivation questionnaire II: Validation with science majors and nonscience majors. Journal of Research in Science Teaching, 48(10), 1159-1176. doi:10.1002/tea.20442

Godwin, A., \& Lee, W. C. (2017). A Cross-sectional Study of Engineering Identity During Undergraduate Education. School of Engineering Education Faculty Publications, Paper 13.

Godwin, A., \& Potvin, G. (2013). Chemical Engineering Students: A Distinct Group among Engineers. Chemical Engineering Education, 47(3), 145-153.

Godwin, A., Potvin, G., \& Hazari, Z. (2013). The Development of Critical Engineering Agency, Identity, and the Impact of Engineering Career Choices. Paper presented at the American Society for Engineering Education Conference and Exposition Atlanta, GA.

Godwin, A., Potvin, G., Hazari, Z., \& Lock, R. M. (2013, 23-26 Oct. 2013). Understanding engineering identity through structural equation modeling. Paper presented at the 2013 IEEE Frontiers in Education Conference (FIE), Oklahoma City, OK. 
Godwin, A., Potvin, G., Hazari, Z., \& Lock, R. M. (2016). Identity, Critical Agency, and Engineering: An Affective Model for Predicting Engineering as a Career Choice. Journal of Engineering Education, 105(2), 312-340. doi:10.1002/jee.20118

Graham, M. J., Frederick, J., Byars-Winston, A., Hunter, A.-B., \& Handelsman, J. (2013). Increasing Persistence of College Students in STEM. Science, 341(6153), 1455. doi:10.1126/science. 1240487

Graven, M., \& Heyd-Metzuyanim, E. (2019). Mathematics identity research: the state of the art and future directions. ZDM, 51(3), 361-377. doi:10.1007/s11858-019-01050-y

Guest, G., Bunce, A., \& Johnson, L. (2006). How Many Interviews Are Enough?:An Experiment with Data Saturation and Variability. Field Methods, 18(1), 59-82. doi: $10.1177 / 1525822 \times 05279903$

Hall, J. (2018). A Phenomenological Study of the Experiences of Successful Women in Science Fields. (Ph.D.), University of Central Florida.

Hancock, G. R. (1999). A sequential Scheffé-type respecification procedure for controlling type I error in exploratory structural equation model modification. Structural Equation Modeling: A Multidisciplinary Journal, 6(2), 158-168. doi:10.1080/10705519909540126

Hancock, G. R., \& Freeman, M. J. (2001). Power and sample size for the root mean square error of approximation test of not close fit in structural equation modeling. Educational and Psychological Measurement, 61(5), 741-758. doi:10.1177/00131640121971491

Hancock, G. R., \& Mueller, R. O. (2001). Rethinking construct reliability within latent variable systems. Paper presented at the Structural equation modeling, present and future : a festschrift in honor of Karl Jöreskog, Uppsala, Sweden.

Harackiewicz, J. M., Durik, A. M., Barron, K. E., Linnenbrink-Garcia, L., \& Tauer, J. M. (2008). The role of achievement goals in the development of interest: Reciprocal relations between achievement goals, interest, and performance. Journal of Educational Psychology, 100(1), 105-122. doi:10.1037/0022-0663.100.1.105

Hazari, Z., Brewe, E., Goertzen, R. M., \& Hodapp, T. (2017). The Importance of High School Physics Teachers for Female Students' Physics Identity and Persistence. The Physics Teacher, 55(2), 96-99. doi:10.1119/1.4974122

Hazari, Z., Sonnert, G., Sadler, P. M., \& Shanahan, M.-C. (2010). Connecting high school physics experiences, outcome expectations, physics identity, and physics career choice: A gender study. Journal of Research in Science Teaching, 47(8), 978-1003. doi:10.1002/tea.20363

Henson, R. K. (2001). Understanding Internal Consistency Reliability Estimates: A Conceptual Primer on Coefficient Alpha. Measurement \& Evaluation in Counseling \& Development, 34(3), 177. doi:10.1080/07481756.2002.12069034

Hesse-Biber, S. N. (2017). The practice of qualitative research: engaging students in the research process ( $3 \mathrm{rd}$ ed.). Thousand Oaks, CA: SAGE Publications, Inc.

Hidi, S., \& Renninger, K. A. (2006). The four-phase model of interest development. Journal of Educational Psychology, 41(2), 111-127. doi:10.1207/s15326985ep4102_4

Hogg, M. A., \& Terry, D. J. (2000). Social Identity and Self-Categorization Processes in Organizational Contexts. The Academy of Management Review, 25(1), 121-140. doi: $10.2307 / 259266$

Hosbein, K. N., \& Barbera, J. (2020). Alignment of theoretically grounded constructs for the measurement of science and chemistry identity. Chemistry Education Research and Practice. doi:10.1039/C9RP00193J

Hu, L. t., \& Bentler, P. M. (1999). Cutoff criteria for fit indexes in covariance structure analysis: Conventional criteria versus new alternatives. Structural Equation Modeling, 6(1), 1-55. doi:10.1080/10705519909540118 
Huck, S. (2012). Reading Statistics and Research (6th ed.). Boston, MA: Pearson.

Hunsu, N. J., Adesope, O., \& Van Wie, B. J. (2017). Engendering situational interest through innovative instruction in an engineering classroom: what really mattered? Instructional Science, 45(6), 789-804. doi:10.1007/s11251-017-9427-z

Hunter, A.-B., Laursen, S. L., \& Seymour, E. (2007). Becoming a scientist: The role of undergraduate research in students' cognitive, personal, and professional development. Science Education, 91(1), 36-74. doi:10.1002/sce.20173

Hutchinson, S. R., \& Olmos, A. (1998). Behavior of descriptive fit indexes in confirmatory factor analysis using ordered categorical data. Structural Equation Modeling: A Multidisciplinary Journal, 5(4), 344-364. doi:10.1080/10705519809540111

Hyater-Adams, S., Fracchiolla, C., Finkelstein, N., \& Hinko, K. (2018). Critical look at physics identity: An operationalized framework for examining race and physics identity. Physical Review Physics Education Research, 14(1), 010132. doi:10.1103/PhysRevPhysEducRes.14.010132

Jackson, P. A., \& Seiler, G. (2013). Science identity trajectories of latecomers to science in college. Journal of Research in Science Teaching, 50(7), 826-857. doi:10.1002/tea.21088

Johansson, A. (2018). The formation of successful physics students: Discourse and identity perspectives on university physics. (Ph.D.), Acta Universitatis Upsaliensis.

Johns, R. (2005). One Size Doesn't Fit All: Selecting Response Scales For Attitude Items. Journal of Elections, Public Opinion and Parties, 15(2), 237-264. doi:10.1080/13689880500178849

Jones, M. G., Corin, E. N., Andre, T., Childers, G. M., \& Stevens, V. (2017). Factors contributing to lifelong science learning: Amateur astronomers and birders. Journal of Research in Science Teaching, 54(3), 412-433. doi:10.1002/tea.21371

Karunanayake, D., \& Nauta, M. M. (2004). The relationship between race and students' identified career role models and perceived role model influence. The Career Development Quarterly, 52(3), 225-234. doi:10.1002/j.2161-0045.2004.tb00644.x

Kim, A. Y., Sinatra, G. M., \& Seyranian, V. (2018). Developing a STEM Identity Among Young Women: A Social Identity Perspective. Review of Educational Research, 88(4), 589-625. doi:10.3102/0034654318779957

Kline, R. B. (2016). Principles and Practice of Structural Equation Modeling (Fourth Ed.). New York, NY: The Guilford Press.

Koenig, R. (2009). Minority Retention Rates in Science Are Sore Spot for Most Universities. Science, 324(5933), 1386-1387. doi:10.1126/science.324_1386a

Komperda, R., Hosbein, K. N., \& Barbera, J. (2018). Evaluation of the influence of wording changes and course type on motivation instrument functioning in chemistry. Chemistry Education Research and Practice. doi:10.1039/C7RP00181A

Komperda, R., Pentecost, T. C., \& Barbera, J. (2018). Moving beyond Alpha: A Primer on Alternative Sources of Single-Administration Reliability Evidence for Quantitative Chemistry Education Research. Journal of Chemical Education, 95(9), 1477-1491. doi:10.1021/acs.jchemed.8b00220

Krapp, A. (2007). An educational-psychological conceptualisation of interest. International Journal for Educational and Vocational Guidance, 7(1), 5-21. doi:10.1007/s10775-0079113-9

Kratwohl, D. R., Bloom, B. S., \& Masia, B. B. (1964). Taxonomy of Educational Objectives, the classification of educational goals-Handbook II: Affective Domain. Philadelphia, PA: David McKay Company, Inc. 
Krosnick, J. A., \& Presser, S. (2010). Question and questionnaire design. In J. D. Wright \& P. V. Marsden (Eds.), Handbook of survey research (2nd ed., pp. 263-314). San Diego, CA: Elselvier.

Kuder, G. F., \& Richardson, M. W. (1937). The theory of the estimation of test reliability. Psychometrika, 2(3), 151-160. doi:10.1007/bf02288391

Laskasky, K. (2018). The Relationship between Secondary Students' Mathematics Identities, Problem Solving, and Self-Regulation. (Ph.D.), Loyola University Chicago.

Lave, J., \& Wenger, E. (2002). Legitimate peripheral participation in communities of practice. In J. Clarke, H. Ann;, R. Harrison, \& F. Reeve (Eds.), Supporting lifelong learning (Vol. 1, pp. 121-136). London, England: Routledge.

Lent, R. W., Brown, S. D., \& Hackett, G. (1994). Toward a Unifying Social Cognitive Theory of Career and Academic Interest, Choice, and Performance. Journal of Vocational Behavior, 45(1), 79-122. doi:10.1006/jvbe.1994.1027

Lent, R. W., Lopez, F. G., Brown, S. D., \& Gore, J. P. A. (1996). Latent Structure of the Sources of Mathematics Self-Efficacy. Journal of Vocational Behavior, 49(3), 292-308. doi:10.1006/jvbe. 1996.0045

Li, S. L., \& Loverude, M. E. (2013). Identity and belonging: Are you a physicist (chemist)? AIP Conference Proceedings, 1513(1), 246-249. doi:10.1063/1.4789698

Linnenbrink-Garcia, L., Durik, A. M., Conley, A. M., Barron, K. E., Tauer, J. M., Karabenick, S. A., \& Harackiewicz, J. M. (2010). Measuring situational interest in academic domains. Educational and Psychological Measurement, 70(4), 647-671. doi:10.1177/0013164409355699

Liu, Y., Ferrell, B., Barbera, J., \& Lewis, J. E. (2017). Development and evaluation of a chemistry-specific version of the academic motivation scale (AMS-Chemistry). Chemistry Education Research and Practice. doi:10.1039/C6RP00200E

Lock, R. M., Castillo, J., Hazari, Z., \& Potvin, G. (2015). Determining strategies that predict physics identity: Emphasizing recognition and interest. Paper presented at the Physics Education Research Conference College Park, MD.

Locke, E. A., Cartledge, N., \& Knerr, C. S. (1970). Studies of the relationship between satisfaction, goal-setting, and performance. Organizational Behavior and Human Performance, 5(2), 135-158. doi:10.1016/0030-5073(70)90011-5

Lockwood, P. (2006). "Someone like me can be successful": Do college students need samegender role models? Psychology of Women Quarterly, 30(1), 36-46. doi:10.1111/j.14716402.2006.00260.x

Lorber, J. (1994). Paradoxes of gender. Binghamton, NY: Yale University Press.

MacCallum, R. C., Roznowski, M., \& Necowitz, L. B. (1992). Model modifications in covariance structure analysis: the problem of capitalization on chance. Psychological Bulletin, 111(3), 490. doi:10.1037/0033-2909.111.3.490

McDonald, R. P. (1999). Test theory: A unified treatment. Mahwah, NJ: Lawrence Erlbaum Associates, Inc.

Monroe, A. M. (2000). Females in Science Education: White Is the Norm and Class, Language, Lifestyle, and Religion Are Nonissues. Journal of Research in Science Teaching, 37(4), 386-387. doi:10.1002/(SICI)1098-2736(200004)37:4<386::AID-TEA6>3.0.CO;2-M

Mueller, R. O., \& Hancock, G. R. (2008). Best practices in structural equation modeling Best practices in quantitative methods. Thousand Oaks, CA: Sage Punlications, Inc.

Nasir, N. i. S., \& Saxe, G. B. (2003). Ethnic and Academic Identities: A Cultural Practice Perspective on Emerging Tensions and Their Management in the Lives of Minority Students. Educational Researcher, 32(5), 14-18. doi:10.3102/0013189X032005014 
National Research Council. (2012). Discipline-based education research: Understanding and improving learning in undergraduate science and engineering. Washington, D.C.: National Academies Press.

Newsom, J. T. (2015). Fundamental concepts of stability and change Longitudinal Structural Equation Modeling: A Comprehensive Introduction. New York, NY: Routledge.

Novick, M. R. (1966). The axioms and principal results of classical test theory. Journal of Mathematical Psychology, 3(1), 1-18. doi:10.1016/0022-2496(66)90002-2

Osborne, J. (1997). Identification with Academics and Academic Success among Community College Students. Community College Review, 25(1), 59-67. doi: $10.1177 / 009155219702500105$

Palmer, E. S. (2018). (Re) Figuring the World of General Chemistry: Possibilities for Participation, Learning, and Identity. (Ph.D.), UC Berkeley.

Paunesku, D., Walton, G. M., Romero, C., Smith, E. N., Yeager, D. S., \& Dweck, C. S. (2015). Mind-Set Interventions Are a Scalable Treatment for Academic Underachievement. Psychological Science, 26(6), 784-793. doi:10.1177/0956797615571017

Pelaez, B. B. (2017). Examining the Relationships between Gender Role Congruity, Identity, and the Choice to Persist for Women in Undergraduate Physics Majors. (Ph.D.), Florida International University.

Penner, A. M. (2015). Gender inequality in science. Science, 347(6219), 234-235. doi:10.1126/science.aaa3781

Potvin, G., \& Hazari, Z. (2013, July 17-18). The Development and Measurement of Identity across the Physical Sciences. Paper presented at the Physics Education Research Conference, Portland, OR.

Preacher, K. J., \& MacCallum, R. C. (2003). Repairing Tom Swift's Electric Factor Analysis Machine. Understanding Statistics, 2(1), 13-43. doi:10.1207/S15328031US0201_02

President's Council of Advisors on Science and Technology. (2012). Engage to Excel: Producing One Million Additional College Graduates with Degrees in Science, Technology, Engineering, and Mathematics. Report to the President. Retrieved from Washington, DC:

Quan, G. (2017). Becoming a Physicist: How Identities and Practices Shape Physics Trajectories. (Ph.D.), University of Maryland.

Quinlan, K. M. (2019). What triggers students' interest during higher education lectures? personal and situational variables associated with situational interest. Studies in Higher Education, 44(10), 1781-1792. doi:10.1080/03075079.2019.1665325

Quon, A. (2018). How Undergraduate Research Experiences Develop Student Science Identity and Views on Becoming Faculty. (Ph.D.), George Mason University.

Renninger, K. A., \& Hidi, S. (2011). Revisiting the Conceptualization, Measurement, and Generation of Interest. Journal of Educational Psychology, 46(3), 168-184. doi:10.1080/00461520.2011.587723

Renninger, K. A., Nieswandt, M., \& Hidi, S. (2015). Interest in mathematics and science learning. Washington, DC: American Educational Research Association.

Rhemtulla, M., Brosseau-Liard, P. É., \& Savalei, V. (2012). When can categorical variables be treated as continuous? A comparison of robust continuous and categorical SEM estimation methods under suboptimal conditions. Psychological methods, 17(3), 354. doi:10.1037/a0029315.

Robbins, S. B., Lauver, K., Le, H., Davis, D., Langley, R., \& Carlstrom, A. (2004). Do Psychosocial and Study Skill Factors Predict College Outcomes? A Meta-Analysis. Psychological Bulletin, 130(2), 261-288. doi:10.1037/0033-2909.130.2.261

Robinson, K. A., Perez, T., Nuttall, A. K., Roseth, C. J., \& Linnenbrink-Garcia, L. (2018). From science student to scientist: Predictors and outcomes of heterogeneous science identity 
trajectories in college. Developmental Psychology, 54(10), 1977-1992.

doi: $10.1037 / \mathrm{dev} 0000567$

Robnett, R. D., Chemers, M. M., \& Zurbriggen, E. L. (2015). Longitudinal associations among undergraduates' research experience, self-efficacy, and identity. Journal of Research in Science Teaching, 52(6), 847-867. doi:10.1002/tea.21221

Rotgans, J. I., \& Schmidt, H. G. (2011). The role of teachers in facilitating situational interest in an active-learning classroom. Teaching and Teacher Education, 27(1), 37-42. doi:10.1016/j.tate.2010.06.025

Rotgans, J. I., \& Schmidt, H. G. (2017). Interest development: Arousing situational interest affects the growth trajectory of individual interest. Contemporary Educational Psychology, 49, 175-184. doi:10.1016/j.cedpsych.2017.02.003

Rust, J., \& Golombok, S. (2014). Modern psychometrics: The science of psychological assessment. New York, NY: Routledge.

Ryan, R. M., \& Deci, E. L. (2000). Self-determination theory and facilitation of intrinsic motivation, social development and well-being. American Psychologist, 55. doi:10.1037/0003-066x.55.1.68

Saldaña, J. (2015). The coding manual for qualitative researchers. Thousand Oaks, CA: Sage.

Salta, K., \& Koulougliotis, D. (2015). Assessing motivation to learn chemistry: adaptation and validation of Science Motivation Questionnaire II with Greek secondary school students. Chemistry Education Research and Practice, 16(2), 237-250. doi:10.1039/C4RP00196F

Sansone, C., Fraughton, T., Zachary, J. L., Butner, J., \& Heiner, C. (2011). Self-regulation of motivation when learning online: the importance of who, why and how. Educ. Technol. Res. Dev., 59(2), 199-212. doi:10.1007/s11423-011-9193-6

Satorra, A., \& Bentler, P. M. (1988). Scaling corrections for chi-square statistics in covariance structure analysis. Paper presented at the Proceedings of the American Statistical Association, Alexandria, VA.

Satorra, A., \& Bentler, P. M. (1994). Corrections to test statistics and standard errors in covariance structure analyis. In A. v. Eye \& C. C. Clogg (Eds.), Latent Variable Analysis: Applications to Developmental Research (pp. 399-419). Newbury Park, CA: Sage.

Satorra, A., \& Bentler, P. M. (2010). Ensuring positiveness of the scaled difference chi-square test statistic. Psychometrika, 75(2), 243-248. doi:10.1007/s11336-009-9135-y

Schermelleh-Engel, K., Moosbrugger, H., \& Müller, H. (2003). Evaluating the fit of structural equation models: Tests of significance and descriptive goodness-of-fit measures. Methods of psychological research online, 8(2), 23-74.

Schiefele, U. (1991). Interest, Learning, and Motivation. Journal of Educational Psychology, 26(3/4), 299. doi:doi.org/10.1080/00461520.1991.9653136

Schiefele, U. (1999). Interest and learning from text. Scientific studies of reading, 3(3), 257-279. doi:10.1207/s1532799xssr0303_4

Schinske, J. N., Perkins, H., Snyder, A., \& Wyer, M. (2016). Scientist spotlight homework assignments shift students' stereotypes of scientists and enhance science identity in a diverse introductory science class. CBE-Life Sciences Education, 15(3). doi:10.1187/cbe.16-01-0002

Sellers, R. M., Smith, M. A., Shelton, J. N., Rowley, S. A. J., \& Chavous, T. M. (1998). Multidimensional Model of Racial Identity: A Reconceptualization of African American Racial Identity. Personality and Social Psychology Review, 2(1), 18-39. doi:10.1207/s15327957pspr0201_2

Seymour, E., Hewitt, N. M., \& Friend, C. M. (1997). Talking about leaving: Why undergraduates leave the sciences (Vol. 12). Boulder, CO: Westview Press. 
Sfard, A., \& Prusak, A. (2005). Telling identities: In search of an analytic tool for investigating learning as a culturally shaped activity. Educational Researcher, 34(4), 14-22. doi:10.3102/0013189X034004014

Shanahan, M. C. (2009). Identity in science learning: exploring the attention given to agency and structure in studies of identity. Stud. Sci. Educ., 45(1), 43-64. doi: $10.1080 / 03057260802681847$

Shapiro, J. R., \& Williams, A. M. (2012). The Role of Stereotype Threats in Undermining Girls' and Women's Performance and Interest in STEM Fields. Sex Roles, 66(3), 175-183. doi:10.1007/s11199-011-0051-0

Shedlosky-Shoemaker, R., \& Fautch, J. M. (2015). Who Leaves, Who Stays? Psychological Predictors of Undergraduate Chemistry Students' Persistence. Journal of Chemical Education, 92(3), 408-414. doi:10.1021/ed500571j

Sijtsma, K. (2008). On the Use, the Misuse, and the Very Limited Usefulness of Cronbach's Alpha. Psychometrika, 74(1), 107. doi:10.1007/s11336-008-9101-0

Silvia, P. J. (2005). What Is Interesting? Exploring the Appraisal Structure of Interest. Emotion, 5(1), 89-102. doi:10.1037/1528-3542.5.1.89

Sivo, S. A., Fan, X., Witta, E. L., \& Willse, J. T. (2006). The Search for "Optimal" Cutoff Properties: Fit Index Criteria in Structural Equation Modeling. J. Exp. Educ., 74(3), 267288. doi:10.3200/JEXE.74.3.267-288

Smith, J. A. (1995). Semi-structured interviewing and qualitative analysis. In J. A. Smith, R. Harré, \& L. Van Langenhove (Eds.), Rethinking methods in psychology (pp. 9-26). London: Sage Publications.

Spradley, J. P. (1980). Participant observation. New York, NY: Wadsworth Thomson Learning.

Sriram, R. (2014). Rethinking Intelligence: The Role of Mindset in Promoting Success for Academically High-Risk Students. Journal of College Student Retention: Research, Theory \& Practice, 15(4), 515-536. doi:10.2190/CS.15.4.c

Steiger, J. H. (1990). Structural Model Evaluation and Modification: An Interval Estimation Approach. Multivariate Behavioral Research, 25(2), 173-180. doi:10.1207/s15327906mbr2502_4

Stets, J. E. (1995). Role Identities and Person Identities: Gender Identity, Mastery Identity, and Controlling One's Partner. Sociological Perspectives, 38(2), 129-150. doi: $10.2307 / 1389287$

Stets, J. E., Brenner, P. S., Burke, P. J., \& Serpe, R. T. (2017). The science identity and entering a science occupation. Soc. Sci. Res., 64, 1-14. doi:10.1016/j.ssresearch.2016.10.016

Stets, J. E., \& Burke, P. J. (2000). Identity theory and social identity theory. Social Psychology Quarterly, 63(3), 224-237. doi:10.2307/2695870

Tate, E. D., \& Linn, M. C. (2005). How Does Identity Shape the Experiences of Women of Color Engineering Students? Journal of Science Education and Technology, 14(5), 483-493. doi:10.1007/s10956-005-0223-1

Tonso, K. L. (2006). Teams that Work: Campus Culture, Engineer Identity, and Social Interactions. Journal of Engineering Education, 95(1), 25-37. doi:10.1002/j.21689830.2006.tb00875.x

Tourangeau, R., \& Yan, T. (2012). Introduction to survey sampling. In H. Cooper, P. M. Camic, D. L. Long, A. T. Panter, D. Rindskopf, \& K. J. Sher (Eds.), APA handbook of research methods in psychology, Vol 2: Research designs: Quantitative, qualitative, neuropsychological, and biological. (pp. 227-251). Washington, DC: American Psychological Association. 
Trujillo, G., \& Tanner, K. D. (2014). Considering the role of affect in learning: monitoring students' self-efficacy, sense of belonging, and science identity. CBE-Life Sciences Education, 13(1), 6-15. doi:10.1187/cbe.13-12-0241

Usher, E. L., \& Pajares, F. (2009). Sources of self-efficacy in mathematics: A validation study. Contemp. Educ. Psychol., 34(1), 89-101. doi:10.1016/j.cedpsych.2008.09.002

Verdín, D., \& Godwin, A. (2017, 18-21 Oct. 2017). Testing for measurement invariance in engineering identity constructs for first-generation college students. Paper presented at the 2017 IEEE Frontiers in Education Conference (FIE), Indianapolis, IN.

Verdín, D., Godwin, A., Kirn, A., Benson, L., \& Potvin, G. (2018, April 29). Understanding How Engineering Identity and Belongingness Predict Grit for First-Generation College Students. Paper presented at the The Collaborative Network for Engineering and Computing Diversity Conference, Crystal City, VA.

Verhoeven, M., Poorthuis, A. M. G., \& Volman, M. (2018). The Role of School in Adolescents' Identity Development. A Literature Review. Educational Psychology Review. doi:10.1007/s10648-018-9457-3

Vincent-Ruz, P. (2019). Conceptualizing Science Identity: Its Nature and the Gendered Role It Plays in Early Secondary Students' Science Choices. (Ph.D.), University of Pittsburgh.

Vincent-Ruz, P., \& Schunn, C. D. (2018). The nature of science identity and its role as the driver of student choices. Int. J. of STEM Educ., 5(1), 48. doi:10.1186/s40594-018-0140-5

Wenger, E. (2000). Communities of Practice and Social Learning Systems. Organization, 7(2), 225-246. doi:10.1177/135050840072002

Willis, G. (2005). Cognitive Interviewing. Thousand Oaks, CA: SAGE Publications.

Yeager, D. S., \& Dweck, C. S. (2012). Mindsets That Promote Resilience: When Students Believe That Personal Characteristics Can Be Developed. Educational Psychologist, 47(4), 302-314. doi:10.1080/00461520.2012.722805

Yu, C. Y., \& Muthén, B. (2002). Evaluation of model fit indices for latent variable models with categorical and continuous outcomes. Paper presented at the American Educational Research Association, New Orleans, LA. 


\section{Appendix A: List of Questions and Demographics Used in Phase One}

Bracketed words were replaced with the word "chemistry" for the discipline-specific version of the question.

\section{Section I: Attributes of a science or chemistry person and self-recognition}

- What do you think makes someone a [science] person?

- Do you see yourself as a [science] person?

- Can you point me to some specifics or examples that would help me understand why you see (or don't see) yourself in this way?

\section{Section II: Recognition by others}

- Do your friends see you as a [science] person?

○ How do you know they see (or don't see you) this way?

- Do your peers see you as a [science] person?

$\circ$ Who do you consider your peers?

○ How do you know they see (or don't see you) this way?

- Do other people who are important to you see you as a [science] person?

- Who did you think of when responding to this question?

○ How do you know they see (or don't see you) this way?

- With regard to others seeing you as a science or chemistry person are their individuals or groups we didn't discuss that you would like to mention?

- Have you had experiences in which you were recognized as a [science] person?

\section{Section III: Performance/Competence}

- How confident are you that you can understand [science] in class?

- What is your confidence based on?

- How confident are you that you can understand [science] outside of class?

- What is your confidence based on?

- Do you understand concepts that you've studied in [science]?

○ What does "understand concepts" mean to you?

- Do you do well on exams in [science]?

$\circ$ What does doing well mean to you?

- Do others ask you for help in [science]?

- Can you give me some examples of who asks you for help?

- Have you overcome any setbacks in [science]?

○ What types? How did you overcome them? 


\section{Section IV: Interest}

- Are you interested in learning more about [science]?

o What about [science] makes you interested/not interested?

- Do you enjoy learning [science]?

○ What about [science do you enjoy/not enjoy?

- Do you find fulfillment in doing [science]?

○ What about doing [science] makes you feel fulfilled?

\section{Demographics}

- What is your university status (e.g., undergrad, transfer student, post-bac)?

- What is the gender you identify as?

- What is the race/ethnicity you identify with? 


\section{Appendix B: Phase Two Additional Information}

Table B.1. Original and revised scale items. Bracketed portion replaced with science or chemistry.

\section{Mastery Experiences}

Original

I make excellent grades on math tests.

I have always been successful with math.

Even when I study very hard, I do poorly in math.

I got good grades in math on my last report card.

I do well on math assignments.

I do well on even the most difficult math assignments.

Verbal Persuasion

Original

My math teachers have told me that I am good at learning math.

People have told me that I have a talent for math. Adults in my family have told me what a good math student I am.

I have been praised for my ability in math. Other students have told me that I'm good at learning math.

My classmates like to work with me in math because they think I'm good at it.

\section{Initial Interest}

Feeling-related

Original

I am fascinated by chemistry.

I chose to take general chemistry because I'm really interested in the topic.

I am really excited about taking this class. I am really looking forward to learning more about chemistry.

Value-related

Original

I think the field of chemistry is an important discipline.

I think that what we will study in General Chemistry will be important for me to know. I think that what we will study in General Chemistry will be worthwhile for me to know. Maintained Interest

\section{Feeling-related}

\section{Original}

What we are learning in chemistry class this semester is fascinating to me.

This semester, I really enjoy the material we cover in class.

I am excited about what we are learning in chemistry class this semester.

To be honest, I don't find the chemistry material we cover in class interesting.

\section{$\underline{\text { Revised }}$}

I get excellent grades on [ ] exams.

I have been successful with [ ] in the past.

Even with I study very hard, I do poorly in [ ].

I have gotten good course grades in [ ].

I do well on non-exam [ ] assignments.

I do well on even the most difficult non-exam [ ] assignment.

Revised

My [ ] instructors have told me that I am good at [ ].

People have told me that I have a talent for [ ].

Someone that is important to me (e.g., a family member, a friend, etc.) has told me what a good [ ] student I am.

I have been praised for my ability in [ ].

Other students have told me that I'm good at [ ].

My classmates or labmates like to work with me in [] because they think I'm good at [].

\section{$\underline{\text { Revised }}$}

I am fascinated by [ ].

I chose to take this class because I'm really

interested in the topic.

same

I am really looking forward to learning more about [ ].

Revised

I think [ ] is important.

I think that what we will study in this class will be important for me to know.

I think that what we will study in this class will be worthwhile for me to know.

\section{$\underline{\text { Revised }}$}

What we are learning in class is fascinating to me.

I really enjoy the [ ] material we cover in this class.

I am excited about what we are learning in this class.

To be honest, I don't find the [ ] material we cover in class interesting. 
Table B. 1 continued.

\begin{tabular}{|c|c|}
\hline \multicolumn{2}{|c|}{ Maintained Interest cont. } \\
\hline \multicolumn{2}{|c|}{ Value-related } \\
\hline Original & $\underline{\text { Revised }}$ \\
\hline $\begin{array}{l}\text { What we are studying in chemistry class is } \\
\text { useful for me to know. }\end{array}$ & $\begin{array}{l}\text { What we are studying in this class is useful for me to } \\
\text { know. }\end{array}$ \\
\hline $\begin{array}{l}\text { The things we are studying in chemistry this } \\
\text { semester are important to me. }\end{array}$ & $\begin{array}{l}\text { The things we are studying in this class are } \\
\text { important to me. }\end{array}$ \\
\hline $\begin{array}{l}\text { What we are learning in chemistry this semester } \\
\text { is important for my future goals. }\end{array}$ & $\begin{array}{l}\text { What we are learning in this class is important for } \\
\text { my future goals. }\end{array}$ \\
\hline $\begin{array}{l}\text { What we are learning in chemistry this semester } \\
\text { can be applied to real life. }\end{array}$ & $\begin{array}{l}\text { What we are learning in this class can be applied to } \\
\text { real life. }\end{array}$ \\
\hline \multicolumn{2}{|c|}{ Identity Item } \\
\hline Original & Revised \\
\hline I see myself as a [ ] person. & same \\
\hline
\end{tabular}


Table B.2. Descriptive statistics for time 1 items by course and wording conditions.

\begin{tabular}{|c|c|c|c|c|c|c|c|c|c|c|}
\hline Item & ${ }^{\mathrm{a} S c a l e}$ & ${ }^{\mathrm{b}}$ Course & ${ }^{c}$ Wording & Mean & $\begin{array}{c}\text { St. } \\
\text { dev. }\end{array}$ & Median & Min & Max & Skew & Kurtosis \\
\hline \multirow{4}{*}{ I think [ ] is important } & \multirow{4}{*}{ II-V } & $\mathrm{GC}$ & $\mathrm{S}$ & 4.73 & 0.47 & 5 & 3 & 5 & -1.40 & 0.77 \\
\hline & & & $\mathrm{C}$ & 4.57 & 0.58 & 5 & 2 & 5 & -1.17 & 1.28 \\
\hline & & $\mathrm{OC}$ & $\mathrm{S}$ & 4.78 & 0.47 & 5 & 3 & 5 & -1.96 & 3.07 \\
\hline & & & $\mathrm{C}$ & 4.63 & 0.56 & 5 & 3 & 5 & -1.21 & 0.48 \\
\hline \multirow{4}{*}{$\begin{array}{l}\text { I think that what we will } \\
\text { study in this class will } \\
\text { be important for me to } \\
\text { know }\end{array}$} & \multirow{4}{*}{ II-V } & $\mathrm{GC}$ & $\mathrm{S}$ & 4.3 & 0.70 & 4 & 2 & 5 & -0.84 & 0.82 \\
\hline & & & $\mathrm{C}$ & 4.28 & 0.70 & 4 & 2 & 5 & -0.81 & 0.68 \\
\hline & & $\mathrm{OC}$ & $\mathrm{S}$ & 4.31 & 0.71 & 4 & 2 & 5 & -0.82 & 0.48 \\
\hline & & & $\mathrm{C}$ & 4.30 & 0.72 & 4 & 2 & 5 & -0.79 & 0.35 \\
\hline \multirow{4}{*}{$\begin{array}{l}\text { I think that what we will } \\
\text { study in this class will } \\
\text { be worthwhile for me to } \\
\text { know }\end{array}$} & \multirow{4}{*}{ II-V } & $\mathrm{GC}$ & $\mathrm{S}$ & 4.31 & 0.67 & 4 & 2 & 5 & -0.69 & 0.32 \\
\hline & & & $\mathrm{C}$ & 4.31 & 0.67 & 4 & 2 & 5 & -0.75 & 0.61 \\
\hline & & $\mathrm{OC}$ & $\mathrm{S}$ & 4.22 & 0.77 & 4 & 1 & 5 & -0.97 & 1.48 \\
\hline & & & $\mathrm{C}$ & 4.20 & 0.78 & 4 & 1 & 5 & -0.93 & 1.33 \\
\hline \multirow{4}{*}{ I am fascinated by [ ] } & \multirow{4}{*}{ II-F } & $\mathrm{GC}$ & $\mathrm{S}$ & 4.44 & 0.71 & 5 & 2 & 5 & -1.15 & 0.95 \\
\hline & & & $\mathrm{C}$ & 3.95 & 0.88 & 4 & 2 & 5 & -0.54 & -0.40 \\
\hline & & $\mathrm{OC}$ & $\mathrm{S}$ & 4.56 & 0.62 & 5 & 3 & 5 & -1.06 & 0.05 \\
\hline & & & $\mathrm{C}$ & 4.11 & 0.85 & 4 & 1 & 5 & -0.85 & 0.42 \\
\hline \multirow{4}{*}{$\begin{array}{l}\text { I chose to take this class } \\
\text { because I'm really } \\
\text { interested in the topic }\end{array}$} & \multirow{4}{*}{ II-F } & $\mathrm{GC}$ & $\mathrm{S}$ & 3.46 & 1.04 & 4 & 1 & 5 & -0.19 & -0.77 \\
\hline & & & $\mathrm{C}$ & 3.45 & 1.03 & 3 & 1 & 5 & -0.17 & -0.76 \\
\hline & & $\mathrm{OC}$ & $\mathrm{S}$ & 3.39 & 1.08 & 3 & 1 & 5 & -0.15 & -0.84 \\
\hline & & & $\mathrm{C}$ & 3.43 & 1.05 & 3 & 1 & 5 & -0.16 & -0.84 \\
\hline \multirow{4}{*}{$\begin{array}{l}\text { I am really excited } \\
\text { about taking this class }\end{array}$} & \multirow{4}{*}{ II-F } & $\mathrm{GC}$ & $\mathrm{S}$ & 3.79 & 0.91 & 4 & 1 & 5 & -0.39 & -0.40 \\
\hline & & & $\mathrm{C}$ & 3.78 & 0.92 & 4 & 1 & 5 & -0.35 & -0.49 \\
\hline & & $\mathrm{OC}$ & $\mathrm{S}$ & 3.79 & 1.07 & 4 & 1 & 5 & -0.56 & -0.54 \\
\hline & & & $\mathrm{C}$ & 3.82 & 1.04 & 4 & 1 & 5 & -0.54 & -0.50 \\
\hline \multirow{4}{*}{$\begin{array}{l}\text { I am really looking } \\
\text { forward to learning } \\
\text { more about [ ] }\end{array}$} & & $\mathrm{GC}$ & $\mathrm{S}$ & 4.39 & 0.63 & 4 & 2 & 5 & -0.67 & 0.14 \\
\hline & & & $\mathrm{C}$ & 4.14 & 0.79 & 4 & 1 & 5 & -0.72 & 0.34 \\
\hline & $11-\mathrm{F}$ & $\mathrm{OC}$ & $\mathrm{S}$ & 4.52 & 0.58 & 5 & 3 & 5 & -0.69 & -0.54 \\
\hline & & & $\mathrm{C}$ & 4.20 & 0.77 & 4 & 2 & 5 & -0.71 & 0.06 \\
\hline & & $\mathrm{GC}$ & $\mathrm{S}$ & 3.45 & 0.87 & 4 & 1 & 5 & -0.33 & -0.12 \\
\hline I get excellent grades on & & & $\mathrm{C}$ & 3.11 & 0.85 & 3 & 1 & 5 & -0.24 & -0.06 \\
\hline [ ] exams & MIE & $\mathrm{OC}$ & $\mathrm{S}$ & 3.67 & 0.97 & 4 & 1 & 5 & -0.40 & -0.47 \\
\hline & & & $\mathrm{C}$ & 3.37 & 1.10 & 3 & 1 & 5 & -0.24 & -0.71 \\
\hline & & $\mathrm{GC}$ & $\mathrm{S}$ & 4.14 & 0.73 & 4 & 1 & 5 & -0.69 & 0.80 \\
\hline I have been successful & $\mathrm{MF}$ & & $\mathrm{C}$ & 3.65 & 0.97 & 4 & 1 & 5 & -0.57 & -0.08 \\
\hline with [ ] in the past & $\mathrm{IVID}$ & $\mathrm{OC}$ & $\mathrm{S}$ & 4.22 & 0.71 & 4 & 1 & 5 & -0.79 & 1.25 \\
\hline & & & $\mathrm{C}$ & 3.91 & 0.90 & 4 & 1 & 5 & -0.85 & 0.76 \\
\hline & & $\mathrm{GC}$ & $\mathrm{S}$ & 3.8 & 0.92 & 4 & 1 & 5 & -0.78 & 0.36 \\
\hline Even when I study very & $\mathrm{ME}$ & & $\mathrm{C}$ & 3.63 & 0.99 & 4 & 1 & 5 & -0.70 & 0.08 \\
\hline hard, I do poorly in [ ] & TIE & $\mathrm{OC}$ & $\mathrm{S}$ & 3.94 & 0.96 & 4 & 1 & 5 & -1.05 & 0.95 \\
\hline & & & $\mathrm{C}$ & 3.73 & 1.10 & 4 & 1 & 5 & -0.65 & -0.39 \\
\hline & & $\mathrm{GC}$ & $\mathrm{S}$ & 4.11 & 0.73 & 4 & 1 & 5 & -0.73 & 1.03 \\
\hline I have gotten good & $\mathrm{ME}$ & & $\mathrm{C}$ & 3.63 & 0.94 & 4 & 1 & 5 & -0.52 & -0.04 \\
\hline course grades in [ ] & VIE & $\mathrm{OC}$ & $\mathrm{S}$ & 4.24 & 0.73 & 4 & 1 & 5 & -1.03 & 1.83 \\
\hline & & & $\mathrm{C}$ & 3.94 & 1.02 & 4 & 1 & 5 & -0.97 & 0.51 \\
\hline & & $\mathrm{GC}$ & $\mathrm{S}$ & 4.04 & 0.66 & 4 & 2 & 5 & -0.23 & -0.08 \\
\hline I do well on non-exam [ & $\mathrm{MF}$ & & $\mathrm{C}$ & 3.89 & 0.72 & 4 & 1 & 5 & -0.33 & 0.25 \\
\hline ] assignments & MIE & $\mathrm{OC}$ & $\mathrm{S}$ & 4.18 & 0.63 & 4 & 1 & 5 & -0.59 & 2.03 \\
\hline & & & $\mathrm{C}$ & 4.04 & 0.69 & 4 & 1 & 5 & -0.78 & 1.82 \\
\hline & & $\mathrm{GC}$ & $\mathrm{S}$ & 3.31 & 0.86 & 3 & 1 & 5 & -0.16 & -0.02 \\
\hline most difficult non-exam & $\mathrm{MF}$ & & $\mathrm{C}$ & 3.18 & 0.86 & 3 & 1 & 5 & -0.03 & 0.22 \\
\hline most dimlicult non-exam & DIE & $\mathrm{OC}$ & $\mathrm{S}$ & 3.62 & 0.85 & 4 & 1 & 5 & -0.58 & 0.24 \\
\hline [ ] assignments & & & $\mathrm{C}$ & 3.46 & 0.85 & 4 & 1 & 5 & -0.39 & 0.12 \\
\hline & & $\mathrm{GC}$ & $\mathrm{S}$ & 3.3 & 0.94 & 3 & 1 & 5 & -0.42 & -0.04 \\
\hline $\begin{array}{l}\text { ny [ ] instructors nave } \\
\text { told me that I am good }\end{array}$ & VP & & $\mathrm{C}$ & 3.01 & 0.92 & 3 & 1 & 5 & -0.24 & 0.16 \\
\hline told me that 1 am good & $\mathrm{VP}$ & $\mathrm{OC}$ & $\mathrm{S}$ & 3.39 & 0.97 & 3 & 1 & 5 & -0.13 & -0.48 \\
\hline & & & $\mathrm{C}$ & 3.14 & 0.97 & 3 & 1 & 5 & 0.01 & -0.25 \\
\hline & & $\mathrm{GC}$ & $\mathrm{S}$ & 3.34 & 1.00 & 3 & 1 & 5 & -0.12 & -0.73 \\
\hline People have told me that & & & $\mathrm{C}$ & 2.89 & 0.91 & 3 & 1 & 5 & 0.13 & -0.01 \\
\hline I have a talent for [ ] & $V P$ & $\mathrm{OC}$ & $\mathrm{S}$ & 3.54 & 0.98 & 4 & 1 & 5 & -0.28 & -0.62 \\
\hline & & & $\mathrm{C}$ & 3.17 & 1.48 & 3 & 1 & 5 & -0.06 & 0.07 \\
\hline
\end{tabular}


Table B.2 continued.

\begin{tabular}{|c|c|c|c|c|c|c|c|c|c|c|}
\hline Item & ${ }^{\text {aScale }}$ & ${ }^{\mathrm{b}}$ Course & ${ }^{\mathrm{c}}$ Wording & Mean & $\begin{array}{c}\text { St. } \\
\text { dev. }\end{array}$ & Median & Min & Max & Skew & Kurtosis \\
\hline \multirow{4}{*}{$\begin{array}{l}\text { Someone that is } \\
\text { important to me (e.g., a } \\
\text { family member, a } \\
\text { friend, etc.) has told me } \\
\text { what a good [ ] student } \\
\text { I am }\end{array}$} & \multirow{4}{*}{ VP } & \multirow[t]{2}{*}{$\mathrm{GC}$} & $\mathrm{S}$ & 3.53 & 1.03 & 4 & 1 & 5 & -0.36 & -0.65 \\
\hline & & & C & 3.11 & 0.96 & 3 & 1 & 5 & 0.07 & -0.33 \\
\hline & & \multirow[t]{2}{*}{$\mathrm{OC}$} & S & 3.86 & 0.98 & 4 & 1 & 5 & -0.69 & -0.13 \\
\hline & & & $\mathrm{C}$ & 3.54 & 0.98 & 4 & 1 & 5 & -0.17 & -0.79 \\
\hline \multirow{4}{*}{$\begin{array}{l}\text { I have been praised for } \\
\text { my ability in [ ] }\end{array}$} & \multirow{4}{*}{ VP } & \multirow[t]{2}{*}{ GC } & $\mathrm{S}$ & 3.33 & 0.99 & 3 & 1 & 5 & -0.38 & -0.4 \\
\hline & & & $\mathrm{C}$ & 2.94 & 0.95 & 3 & 1 & 5 & -0.14 & -0.48 \\
\hline & & \multirow[t]{2}{*}{$\mathrm{OC}$} & $\mathrm{S}$ & 3.54 & 0.95 & 4 & 1 & 5 & -0.31 & -0.23 \\
\hline & & & $\mathrm{C}$ & 3.24 & 0.97 & 3 & 1 & 5 & 0.01 & -0.50 \\
\hline \multirow{4}{*}{$\begin{array}{l}\text { Other students have } \\
\text { told me that I'm good at } \\
\text { [ ] }\end{array}$} & \multirow{4}{*}{ VP } & \multirow[t]{2}{*}{$\mathrm{GC}$} & $\mathrm{S}$ & 3.52 & 0.97 & 4 & 1 & 5 & -0.28 & -0.65 \\
\hline & & & $\mathrm{C}$ & 3.18 & 0.92 & 3 & 1 & 5 & -0.11 & -0.25 \\
\hline & & \multirow[t]{2}{*}{$\mathrm{OC}$} & $\mathrm{S}$ & 3.72 & 0.89 & 4 & 1 & 5 & -0.40 & -0.37 \\
\hline & & & $\mathrm{C}$ & 3.54 & 0.92 & 4 & 1 & 5 & -0.30 & -0.20 \\
\hline \multirow{4}{*}{$\begin{array}{l}\text { My classmates or } \\
\text { labmates like to work } \\
\text { with me in [ ] because } \\
\text { they think I'm good at [ } \\
\text { ] }\end{array}$} & \multirow{4}{*}{ VP } & \multirow[t]{2}{*}{$\mathrm{GC}$} & $\mathrm{S}$ & 3.37 & 0.80 & 3 & 1 & 5 & -0.11 & 0.09 \\
\hline & & & $\mathrm{C}$ & 3.19 & 0.74 & 3 & 1 & 5 & -0.01 & 0.51 \\
\hline & & \multirow[t]{2}{*}{$\mathrm{OC}$} & $\mathrm{S}$ & 3.62 & 0.85 & 4 & 1 & 5 & -0.19 & -0.16 \\
\hline & & & $\mathrm{C}$ & 3.45 & 0.86 & 3 & 1 & 5 & -0.07 & -0.10 \\
\hline \multirow{4}{*}{$\begin{array}{l}\text { I see myself as a [ ] } \\
\text { person }\end{array}$} & \multirow{4}{*}{ Identity } & \multirow[t]{2}{*}{$\mathrm{GC}$} & $\mathrm{S}$ & 3.94 & 0.92 & 4 & 1 & 5 & -0.66 & 0.00 \\
\hline & & & $\mathrm{C}$ & 3.15 & 0.96 & 3 & 1 & 5 & 0.07 & -0.46 \\
\hline & & \multirow[t]{2}{*}{$\mathrm{OC}$} & $\mathrm{S}$ & 4.23 & 0.80 & 4 & 1 & 5 & -1.17 & 2.21 \\
\hline & & & $\mathrm{C}$ & 3.28 & 1.03 & 3 & 1 & 5 & -0.02 & -0.59 \\
\hline
\end{tabular}

${ }^{\mathrm{a}} \mathrm{II}-\mathrm{V}=$ Initial Interest Value-related, II-F $=$ Initial Interest Feeling-related, $\mathrm{ME}=$ Mastery Experiences, $\mathrm{VP}$ $=$ Verbal Persuasion, ${ }^{\mathrm{b}} \mathrm{GC}=$ General Chemistry, $\mathrm{OC}=$ Organic Chemistry, ${ }^{\mathrm{c}} \mathrm{S}=$ Science, $\mathrm{C}=$ Chemistry 
Table B.3. Descriptive statistics for time 2 items by course and wording conditions.

\begin{tabular}{|c|c|c|c|c|c|c|c|c|c|c|}
\hline Item & ${ }^{\mathrm{a}}$ Scale & ${ }^{\mathrm{b}}$ Course & ${ }^{\mathrm{c}}$ Wording & Mean & $\begin{array}{c}\text { St. } \\
\text { dev. }\end{array}$ & Median & Min & Max & Skew & Kurtosis \\
\hline \multirow{4}{*}{$\begin{array}{l}\text { What we are studying in } \\
\text { this class is useful for me } \\
\text { to know }\end{array}$} & \multirow{4}{*}{ MI-V } & $\mathrm{GC}$ & $S$ & 3.88 & 0.85 & 4 & 1 & 5 & -0.85 & 1.02 \\
\hline & & & $\mathrm{C}$ & 3.91 & 0.84 & 4 & 1 & 5 & -0.88 & 1.16 \\
\hline & & $\mathrm{OC}$ & S & 3.99 & 0.92 & 4 & 1 & 5 & -0.87 & 0.51 \\
\hline & & & $\mathrm{C}$ & 4.01 & 0.90 & 4 & 1 & 5 & -0.88 & 0.63 \\
\hline \multirow{4}{*}{$\begin{array}{l}\text { The things we are studying } \\
\text { in this class are important } \\
\text { to me }\end{array}$} & \multirow{4}{*}{ MI-V } & GC & $\mathrm{S}$ & 3.74 & 0.95 & 4 & 1 & 5 & -0.65 & 0.17 \\
\hline & & & $\mathrm{C}$ & 3.78 & 0.93 & 4 & 1 & 5 & -0.68 & 0.22 \\
\hline & & $\mathrm{OC}$ & S & 3.85 & 0.95 & 4 & 1 & 5 & -0.67 & 0.14 \\
\hline & & & $\mathrm{C}$ & 3.88 & 0.93 & 4 & 1 & 5 & -0.71 & 0.31 \\
\hline \multirow{4}{*}{$\begin{array}{l}\text { What we are learning in } \\
\text { this class is important for } \\
\text { my future goals }\end{array}$} & \multirow{4}{*}{ MI-V } & GC & $\mathrm{S}$ & 3.90 & 1.00 & 4 & 1 & 5 & -0.76 & -0.02 \\
\hline & & & $\mathrm{C}$ & 3.94 & 0.98 & 4 & 1 & 5 & -0.82 & 0.13 \\
\hline & & $\mathrm{OC}$ & S & 4.12 & 0.89 & 4 & 1 & 5 & -1.23 & 1.95 \\
\hline & & & $\mathrm{C}$ & 4.12 & 0.90 & 4 & 1 & 5 & -1.33 & 2.29 \\
\hline \multirow{4}{*}{$\begin{array}{l}\text { What we are learning in } \\
\text { this class can be applied to } \\
\text { real life }\end{array}$} & \multirow{4}{*}{ MI-V } & $\mathrm{GC}$ & $\mathrm{S}$ & 3.81 & 0.89 & 4 & 1 & 5 & -0.73 & 0.48 \\
\hline & & & $\mathrm{C}$ & 3.84 & 0.88 & 4 & 1 & 5 & -0.72 & 0.43 \\
\hline & & $\mathrm{OC}$ & S & 3.95 & 0.91 & 4 & 1 & 5 & -0.78 & 0.26 \\
\hline & & & $\mathrm{C}$ & 3.96 & 0.90 & 4 & 1 & 5 & -0.85 & 0.45 \\
\hline \multirow{4}{*}{$\begin{array}{l}\text { What we are learning in } \\
\text { class is fascinating to me }\end{array}$} & \multirow{4}{*}{ MI-F } & $\mathrm{GC}$ & $\mathrm{S}$ & 3.59 & 1.02 & 4 & 1 & 5 & -0.65 & 0.06 \\
\hline & & & $\mathrm{C}$ & 3.63 & 1.01 & 4 & 1 & 5 & -0.67 & -0.56 \\
\hline & & $\mathrm{OC}$ & $\mathrm{S}$ & 3.77 & 0.99 & 4 & 1 & 5 & -0.49 & 0.17 \\
\hline & & & $\mathrm{C}$ & 3.83 & 0.97 & 4 & 1 & 5 & -0.53 & -0.42 \\
\hline \multirow{4}{*}{$\begin{array}{l}\text { I really enjoy the [ ] } \\
\text { material we cover in this } \\
\text { class }\end{array}$} & \multirow{4}{*}{ MI-F } & GC & $\mathrm{S}$ & 3.73 & 0.89 & 4 & 1 & 5 & -0.63 & 0.37 \\
\hline & & & C & 3.66 & 0.91 & 4 & 1 & 5 & -0.51 & 0.09 \\
\hline & & $\mathrm{OC}$ & S & 3.88 & 0.89 & 4 & 1 & 5 & -0.54 & -0.21 \\
\hline & & & $\mathrm{C}$ & 3.82 & 0.93 & 4 & 1 & 5 & -0.70 & 0.27 \\
\hline \multirow{4}{*}{$\begin{array}{l}\text { I am excited about what we } \\
\text { are learning in class }\end{array}$} & \multirow{4}{*}{ MI-F } & GC & S & 3.60 & 0.93 & 4 & 1 & 5 & -0.38 & -0.24 \\
\hline & & & $\mathrm{C}$ & 3.62 & 0.93 & 4 & 1 & 5 & -0.44 & -0.12 \\
\hline & & $\mathrm{OC}$ & $\mathrm{S}$ & 3.75 & 0.97 & 4 & 1 & 5 & -0.42 & -0.56 \\
\hline & & & $\mathrm{C}$ & 3.79 & 0.94 & 4 & 1 & 5 & -0.47 & -0.36 \\
\hline \multirow{4}{*}{$\begin{array}{l}\text { To be honest, I don't find } \\
\text { the [ ] material we cover in } \\
\text { class interesting }\end{array}$} & & GC & S & 3.63 & 1.05 & 4 & 1 & 5 & -0.58 & -0.33 \\
\hline & & & $\mathrm{C}$ & 3.57 & 1.10 & 4 & 1 & 5 & -0.54 & -0.52 \\
\hline & MI-F & $\mathrm{OC}$ & S & 3.95 & 0.94 & 4 & 1 & 5 & -0.93 & 0.51 \\
\hline & & & $\mathrm{C}$ & 3.85 & 1.02 & 4 & 1 & 5 & -0.79 & 0.01 \\
\hline & & GC & $\mathrm{S}$ & 3.36 & 0.98 & 3 & 1 & 5 & -0.35 & -0.33 \\
\hline I get excellent grades on [ ] & $\mathrm{ME}$ & & $\mathrm{C}$ & 3.02 & 1.10 & 3 & 1 & 5 & -0.05 & -0.74 \\
\hline exams & & $\mathrm{OC}$ & $\mathrm{S}$ & 3.61 & 0.93 & 4 & 1 & 5 & -0.5 & 0.00 \\
\hline & & & $\mathrm{C}$ & 3.22 & 1.04 & 3 & 1 & 5 & -0.11 & -0.56 \\
\hline & & GC & $\mathrm{S}$ & 4.08 & 0.76 & 4 & 1 & 5 & -0.86 & 1.13 \\
\hline I have been successful with & $\mathrm{MF}$ & & $\mathrm{C}$ & 3.63 & 0.97 & 4 & 1 & 5 & -0.54 & -0.14 \\
\hline [ ] in the past & $\mathrm{ME}$ & $\mathrm{OC}$ & $\mathrm{S}$ & 4.28 & 0.72 & 4 & 1 & 5 & -1.12 & 2.24 \\
\hline & & & $\mathrm{C}$ & 3.93 & 0.93 & 4 & 1 & 5 & -0.93 & 0.58 \\
\hline & & GC & $\mathrm{S}$ & 3.64 & 1.05 & 4 & 1 & 5 & -0.67 & -0.08 \\
\hline Even when I study very & $\mathrm{ME}$ & & $\mathrm{C}$ & 3.35 & 1.22 & 4 & 1 & 5 & -0.38 & -0.88 \\
\hline hard, I do poorly in [ ] & $\mathrm{ME}$ & $\mathrm{OC}$ & $\mathrm{S}$ & 3.77 & 0.99 & 4 & 1 & 5 & -1.01 & 0.78 \\
\hline & & & $\mathrm{C}$ & 3.59 & 1.12 & 4 & 1 & 5 & -0.65 & -0.40 \\
\hline & & $\mathrm{GC}$ & $\mathrm{S}$ & 3.96 & 0.80 & 4 & 1 & 5 & -0.72 & 0.73 \\
\hline I have gotten good course & & & $\mathrm{C}$ & 3.60 & 0.96 & 4 & 1 & 5 & -0.52 & -0.21 \\
\hline grades in [ ] & $\mathrm{ME}$ & $\mathrm{OC}$ & $\mathrm{S}$ & 4.13 & 0.80 & 4 & 1 & 5 & -1.19 & 2.16 \\
\hline & & & $\mathrm{C}$ & 3.91 & 0.92 & 4 & 1 & 5 & -0.87 & 0.63 \\
\hline & & $\mathrm{GC}$ & $\mathrm{S}$ & 4.08 & 0.73 & 4 & 1 & 5 & -0.73 & 1.31 \\
\hline I do well on non-exam [ ] & $\mathrm{MF}$ & & $\mathrm{C}$ & 4.01 & 0.74 & 4 & 1 & 5 & -0.58 & 0.62 \\
\hline assignments & ME & $\mathrm{OC}$ & $\mathrm{S}$ & 4.17 & 0.70 & 4 & 1 & 5 & -0.96 & 2.28 \\
\hline & & & $\mathrm{C}$ & 4.04 & 0.73 & 4 & 1 & 5 & -0.68 & 1.09 \\
\hline & & $\mathrm{GC}$ & $\mathrm{S}$ & 3.51 & 0.91 & 4 & 1 & 5 & -0.38 & -0.07 \\
\hline I do well on even the most & & & $\mathrm{C}$ & 3.42 & 0.92 & 3 & 1 & 5 & -0.32 & -0.09 \\
\hline difficult non- & $\mathrm{ME}$ & $\mathrm{OC}$ & $\mathrm{S}$ & 3.68 & 0.89 & 4 & 1 & 5 & -0.53 & 0.07 \\
\hline & & & $\mathrm{C}$ & 3.50 & 0.92 & 4 & 1 & 5 & -0.34 & -0.26 \\
\hline
\end{tabular}


Table B. 3 continued.

\begin{tabular}{|c|c|c|c|c|c|c|c|c|c|c|}
\hline Item & ${ }^{\mathrm{a}}$ Scale & ${ }^{\mathrm{b}}$ Course & ${ }^{\mathrm{c}}$ Wording & Mean & St. dev. & Median & Min & Max & Skew & Kurtosis \\
\hline \multirow{4}{*}{$\begin{array}{l}\text { My [ ] instructors have told } \\
\text { me that I am good at [ ] }\end{array}$} & \multirow{4}{*}{ VP } & GC & $\mathrm{S}$ & 3.20 & 0.96 & 3 & 1 & 5 & -0.16 & -0.41 \\
\hline & & & $\mathrm{C}$ & 2.95 & 0.88 & 3 & 1 & 5 & 0.02 & 0.16 \\
\hline & & $\mathrm{OC}$ & S & 3.30 & 1.05 & 3 & 1 & 5 & -0.18 & -0.67 \\
\hline & & & $\mathrm{C}$ & 3.08 & 0.99 & 3 & 1 & 5 & 0.08 & -0.37 \\
\hline \multirow{4}{*}{$\begin{array}{l}\text { People have told me that I } \\
\text { have a talent for [ ] }\end{array}$} & \multirow{4}{*}{ VP } & GC & $\mathrm{S}$ & 3.36 & 0.95 & 3 & 1 & 5 & -0.25 & -0.42 \\
\hline & & & $\mathrm{C}$ & 2.99 & 0.97 & 3 & 1 & 5 & 0.03 & -0.23 \\
\hline & & $\mathrm{OC}$ & S & 3.70 & 0.95 & 4 & 1 & 5 & -0.45 & -0.56 \\
\hline & & & $\mathrm{C}$ & 3.30 & 1.00 & 3 & 1 & 5 & -0.08 & -0.62 \\
\hline \multirow{4}{*}{$\begin{array}{l}\text { Someone that is important } \\
\text { to me (e.g., a family } \\
\text { member, a friend, etc.) has } \\
\text { told me what a good [ ] } \\
\text { student I am }\end{array}$} & \multirow{4}{*}{ VP } & GC & $S$ & 3.60 & 1.01 & 4 & 1 & 5 & -0.59 & -0.28 \\
\hline & & & $\mathrm{C}$ & 3.21 & 1.00 & 3 & 1 & 5 & 0.04 & -0.71 \\
\hline & & $\mathrm{OC}$ & S & 3.87 & 0.97 & 4 & 1 & 5 & -0.72 & -0.05 \\
\hline & & & $\mathrm{C}$ & 3.55 & 1.06 & 4 & 1 & 5 & -0.38 & -0.59 \\
\hline \multirow{4}{*}{$\begin{array}{l}\text { I have been praised for my } \\
\text { ability in [ ] }\end{array}$} & \multirow{4}{*}{ VP } & $\mathrm{GC}$ & $\mathrm{S}$ & 3.36 & 1.00 & 3 & 1 & 5 & -0.31 & -0.44 \\
\hline & & & $\mathrm{C}$ & 3.06 & 1.00 & 3 & 1 & 5 & -0.05 & -0.38 \\
\hline & & $\mathrm{OC}$ & S & 3.67 & 0.97 & 4 & 1 & 5 & -0.46 & -0.40 \\
\hline & & & $\mathrm{C}$ & 3.33 & 1.00 & 3 & 1 & 5 & -0.18 & -0.47 \\
\hline \multirow{4}{*}{$\begin{array}{l}\text { Other students have told } \\
\text { me that I'm good at [ ] }\end{array}$} & \multirow{4}{*}{ VP } & GC & $\mathrm{S}$ & 3.50 & 0.97 & 4 & 1 & 5 & -0.42 & -0.34 \\
\hline & & & $\mathrm{C}$ & 3.32 & 1.02 & 3 & 1 & 5 & -0.22 & -0.55 \\
\hline & & $\mathrm{OC}$ & $\mathrm{S}$ & 3.87 & 0.82 & 4 & 1 & 5 & -0.73 & 0.99 \\
\hline & & & $\mathrm{C}$ & 3.59 & 0.93 & 4 & 1 & 5 & -0.40 & -0.18 \\
\hline \multirow{4}{*}{$\begin{array}{l}\text { My classmates or labmates } \\
\text { like to work with me in [ ] } \\
\text { because they think I'm } \\
\text { good at [ ] }\end{array}$} & \multirow{4}{*}{ VP } & $\mathrm{GC}$ & $\mathrm{S}$ & 3.47 & 0.87 & 3 & 1 & 5 & -0.16 & -0.10 \\
\hline & & & $\mathrm{C}$ & 3.41 & 0.86 & 3 & 1 & 5 & -0.08 & -0.07 \\
\hline & & $\mathrm{OC}$ & $\mathrm{S}$ & 3.74 & 0.76 & 4 & 1 & 5 & -0.37 & 0.52 \\
\hline & & & $\mathrm{C}$ & 3.53 & 0.84 & 4 & 1 & 5 & -0.19 & 0.04 \\
\hline \multirow{4}{*}{ I see myself as a [ ] person } & \multirow{4}{*}{ Identity } & GC & $\mathrm{S}$ & 3.85 & 0.97 & 4 & 1 & 5 & -0.89 & 0.63 \\
\hline & & & $\mathrm{C}$ & 3.02 & 1.05 & 3 & 1 & 5 & -0.05 & -0.56 \\
\hline & & $\mathrm{OC}$ & $\mathrm{S}$ & 4.18 & 0.82 & 4 & 1 & 5 & -1.03 & 1.34 \\
\hline & & & C & 3.42 & 1.04 & 3 & 1 & 5 & -0.17 & -0.64 \\
\hline
\end{tabular}

${ }^{\mathrm{a}} \mathrm{MI}-\mathrm{V}=$ Maintained Interest Value-related, $\mathrm{mI}-\mathrm{F}=$ Maintained Interest Feeling-related, $\mathrm{ME}=$ Mastery Experiences, $\mathrm{VP}=$ Verbal Persuasion ${ }^{\mathrm{b}} \mathrm{GC}=$ General Chemistry, $\mathrm{OC}=$ Organic Chemistry ${ }^{\mathrm{c}} \mathrm{S}=$ Science, $\mathrm{C}$

$=$ Chemistry 
Table B.4. Congeneric model fit of single-factor scales

\begin{tabular}{|c|c|c|c|c|c|c|c|c|}
\hline Scale & Course $^{a}$ & Wording $^{\mathrm{b}}$ & $\mathrm{df}$ & $\chi^{2}$ & CFI & RMSEA & $\begin{array}{c}\text { RMSEA } \\
{[90 \% \mathrm{CI}]}\end{array}$ & SRMR \\
\hline \multirow{4}{*}{$\begin{array}{l}\text { Initial feeling- } \\
\text { related interest } \\
\quad(\text { Time } 1)\end{array}$} & \multirow{2}{*}{ GC } & $\mathrm{S}(\mathrm{N}=335)$ & 1 & $0.333 \neq$ & 1.00 & 0.00 & {$[0.00,0.00]$} & 0.003 \\
\hline & & $\mathrm{C}(\mathrm{N}=341)$ & 2 & $7.63 *$ & 0.99 & 0.10 & {$[0.03,0.19]$} & 0.02 \\
\hline & \multirow{2}{*}{$\mathrm{OC}$} & $\mathrm{S}(\mathrm{N}=225)$ & 1 & $0.025 \ddagger$ & 1.00 & 0.00 & {$[0.00,0.00]$} & 0.001 \\
\hline & & $\mathrm{C}(\mathrm{N}=226)$ & 2 & $5.87 *$ & 0.99 & 0.11 & {$[\mathrm{NA}, 0.21]$} & 0.02 \\
\hline \multirow{4}{*}{$\begin{array}{c}\text { Mastery } \\
\text { experiences } \\
\text { (Time 1) }\end{array}$} & \multirow{2}{*}{ GC } & $\mathrm{S}(\mathrm{N}=335)$ & 4 & $5.54 \neq$ & 1.00 & 0.04 & {$[0.00,0.11]$} & 0.02 \\
\hline & & $\mathrm{C}(\mathrm{N}=341)$ & 4 & $11.4^{*}$ & 0.99 & 0.08 & {$[0.03,0.14]$} & 0.03 \\
\hline & \multirow{2}{*}{$\mathrm{OC}$} & $\mathrm{S}(\mathrm{N}=225)$ & 4 & $10.0 *$ & 0.98 & 0.09 & {$[0.02,0.16]$} & 0.04 \\
\hline & & $\mathrm{C}(\mathrm{N}=226)$ & 4 & $8.04 *$ & 0.99 & 0.07 & {$[0.00,0.15]$} & 0.03 \\
\hline \multirow{4}{*}{$\begin{array}{c}\text { Verbal } \\
\text { persuasion } \\
\text { (Time 1) }\end{array}$} & \multirow{2}{*}{ GC } & $\mathrm{S}(\mathrm{N}=335)$ & 9 & $27.6^{*}$ & 0.98 & 0.09 & {$[0.06,0.14]$} & 0.03 \\
\hline & & $\mathrm{C}(\mathrm{N}=341)$ & 9 & $12.2 \ddagger$ & 1.00 & 0.04 & {$[0.00,0.09]$} & 0.02 \\
\hline & \multirow{2}{*}{$\mathrm{OC}$} & $\mathrm{S}(\mathrm{N}=225)$ & 9 & $27.6^{*}$ & 0.98 & 0.09 & {$[0.06,0.14]$} & 0.03 \\
\hline & & $\mathrm{C}(\mathrm{N}=226)$ & 9 & $18.0 *$ & 0.99 & 0.08 & {$[0.02,0.13]$} & 0.03 \\
\hline \multirow{4}{*}{$\begin{array}{c}\text { Maintained } \\
\text { feeling-related } \\
\text { interest } \\
\text { (Time 2) }\end{array}$} & \multirow{2}{*}{ GC } & $\mathrm{S}(\mathrm{N}=335)$ & 2 & $2.40 \neq$ & 1.00 & 0.03 & {$[0.00,0.12]$} & 0.008 \\
\hline & & $\mathrm{C}(\mathrm{N}=341)$ & 2 & $0.480 \neq$ & 1.00 & 0.00 & {$[0.00,0.07]$} & 0.003 \\
\hline & \multirow{2}{*}{$\mathrm{OC}$} & $\mathrm{S}(\mathrm{N}=225)$ & 2 & $5.76 \neq$ & 0.99 & 0.10 & {$[\mathrm{NA}, 0.20]$} & 0.02 \\
\hline & & $\mathrm{C}(\mathrm{N}=226)$ & 2 & $0.738 \neq$ & 1.00 & 0.00 & {$[0.00,0.10]$} & 0.006 \\
\hline \multirow{4}{*}{$\begin{array}{c}\text { Mastery } \\
\text { experiences } \\
\text { (Time 2) }\end{array}$} & \multirow{2}{*}{ GC } & $\mathrm{S}(\mathrm{N}=335)$ & 4 & $3.47 \neq$ & 1.00 & 0.00 & {$[0.00,0.09]$} & 0.02 \\
\hline & & $\mathrm{C}(\mathrm{N}=341)$ & 4 & $4.11 \neq$ & 1.00 & 0.01 & {$[0.00,0.09]$} & 0.02 \\
\hline & \multirow{2}{*}{$\mathrm{OC}$} & $\mathrm{S}(\mathrm{N}=225)$ & 4 & $4.08 \neq$ & 1.00 & 0.01 & {$[0.00,0.11]$} & 0.021 \\
\hline & & $\mathrm{C}(\mathrm{N}=226)$ & 4 & $1.17 \neq$ & 1.00 & 0.00 & {$[0.00,0.05]$} & 0.01 \\
\hline \multirow{4}{*}{$\begin{array}{c}\text { Verbal } \\
\text { Persuasion } \\
\text { (Time 2) }\end{array}$} & \multirow{2}{*}{$\mathrm{GC}$} & $\mathrm{S}(\mathrm{N}=335)$ & 9 & $13.6 \neq$ & 1.00 & 0.05 & {$[0.00,0.09]$} & 0.02 \\
\hline & & $\mathrm{C}(\mathrm{N}=341)$ & 9 & $13.3 \neq$ & 0.99 & 0.05 & {$[0.00,0.10]$} & 0.02 \\
\hline & \multirow{2}{*}{$\mathrm{OC}$} & $\mathrm{S}(\mathrm{N}=225)$ & 9 & $20.6^{*}$ & 0.98 & 0.08 & {$[0.04,0.13]$} & 0.03 \\
\hline & & $\mathrm{C}(\mathrm{N}=226)$ & 9 & $27.4^{*}$ & 0.97 & 0.10 & {$[0.06,0.15]$} & 0.03 \\
\hline
\end{tabular}

${ }^{\mathrm{a}} \mathrm{GC}=$ General Chemistry, OC $=$ Organic Chemistry, ${ }^{\mathrm{b}} \mathrm{S}=$ Science, $\mathrm{C}=$ Chemistry.

$* 0.001 \leq p \leq 0.05$. $\neq p \geq 0.05$ 
A)

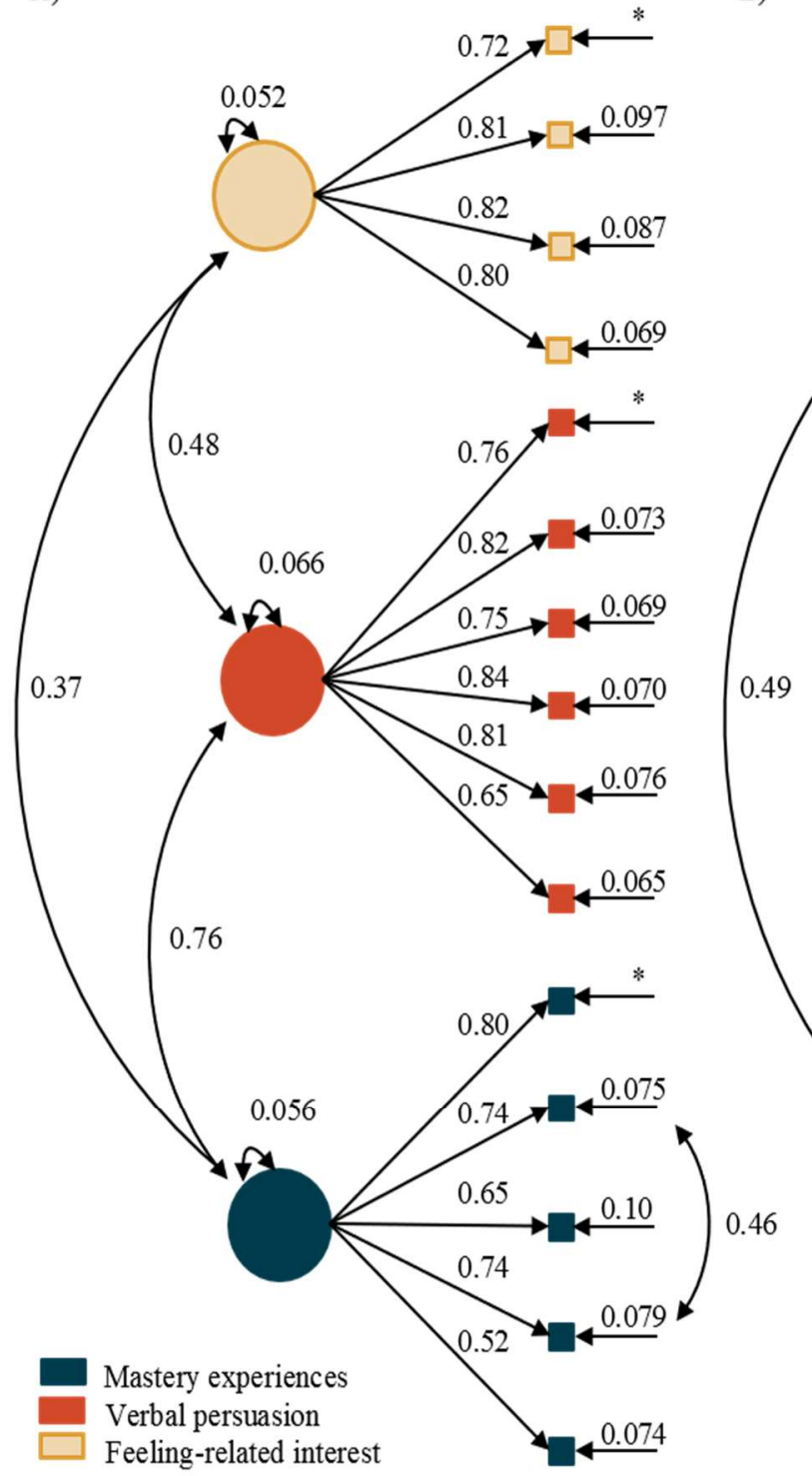

B)

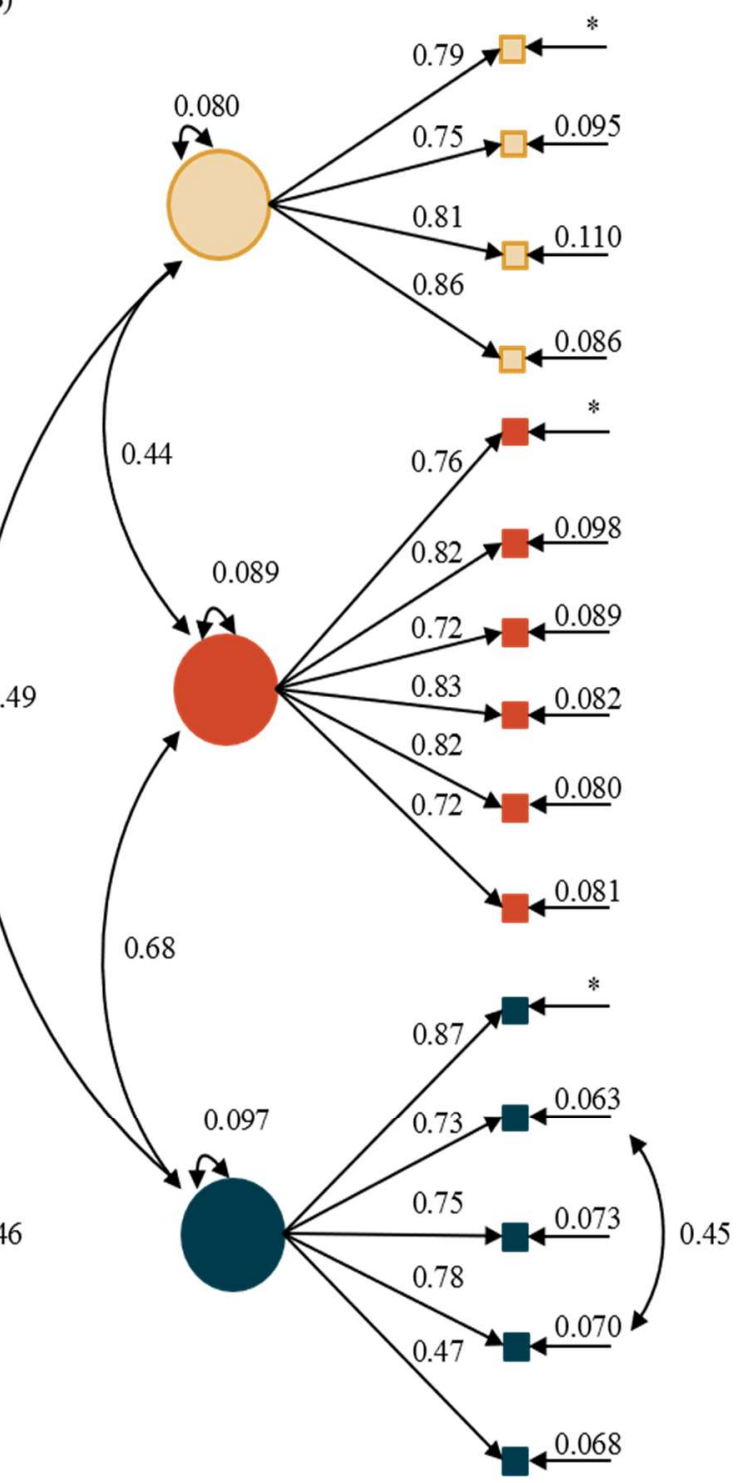

Figure B.1. Localized estimates for time 1, chemistry worded, three-factor correlated models within A) general and B) organic chemistry. *Indicates the reference variable for the model. 
A)

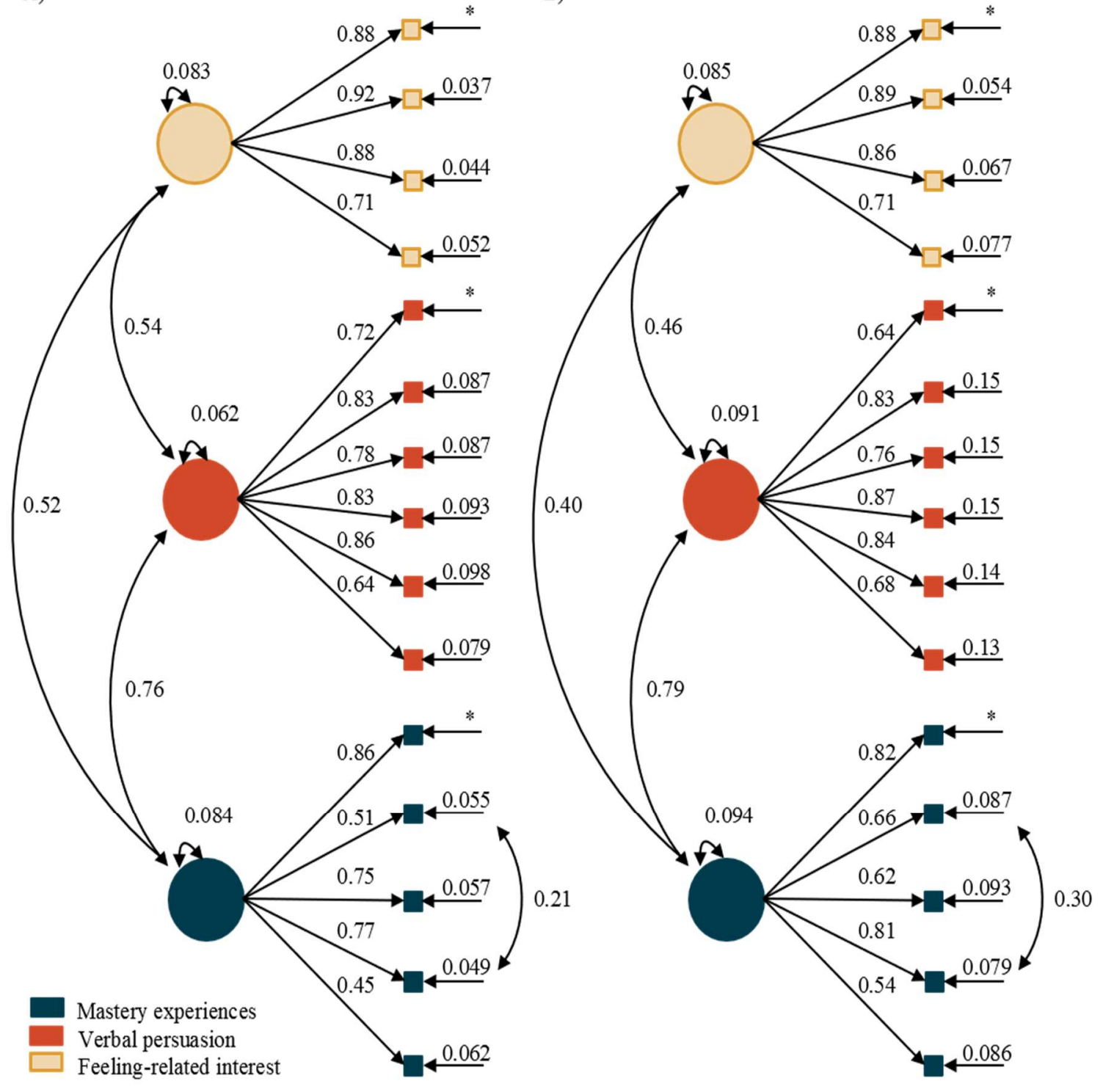

Figure B.2. Localized estimates for time 2, chemistry worded, three-factor correlated models within A) general and B) organic chemistry. *Indicates the reference variable for the model. 
A)

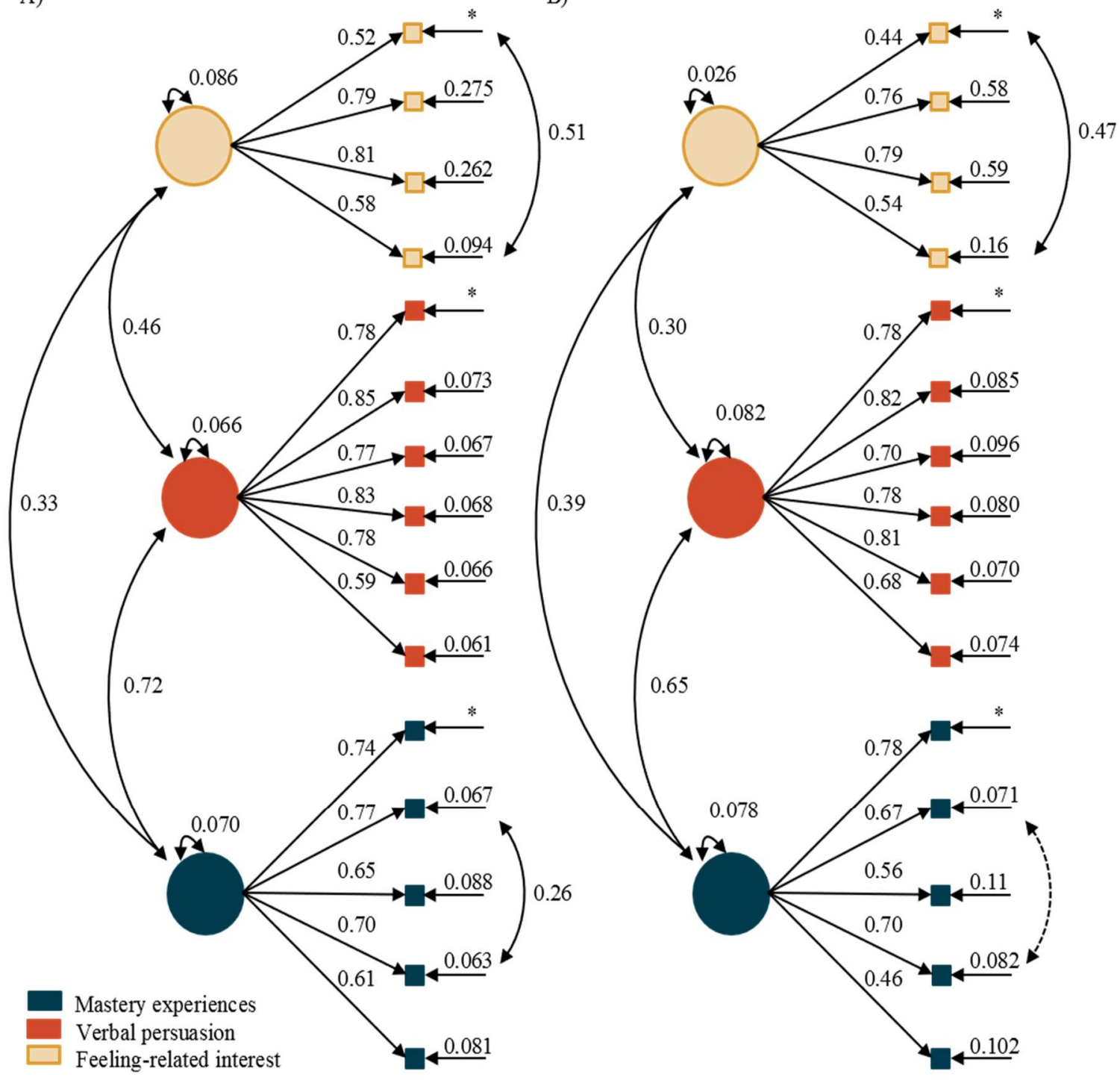

Figure B.3. Localized estimates for time 1, science worded, three-factor correlated models within A) general and B) organic chemistry. Dashed line indicates nonsignificant path. *Indicates the reference variable for the model. 
A)

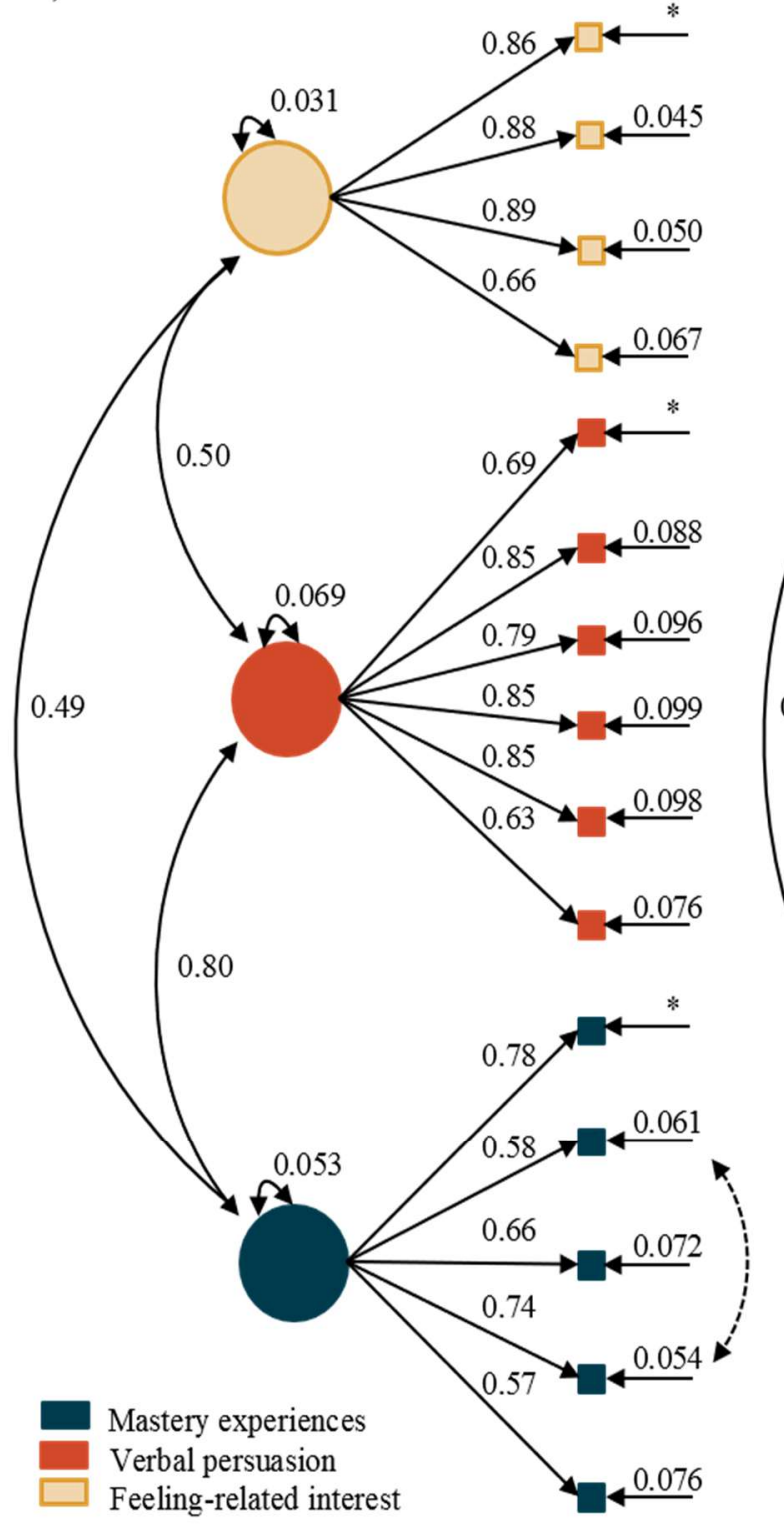

B)

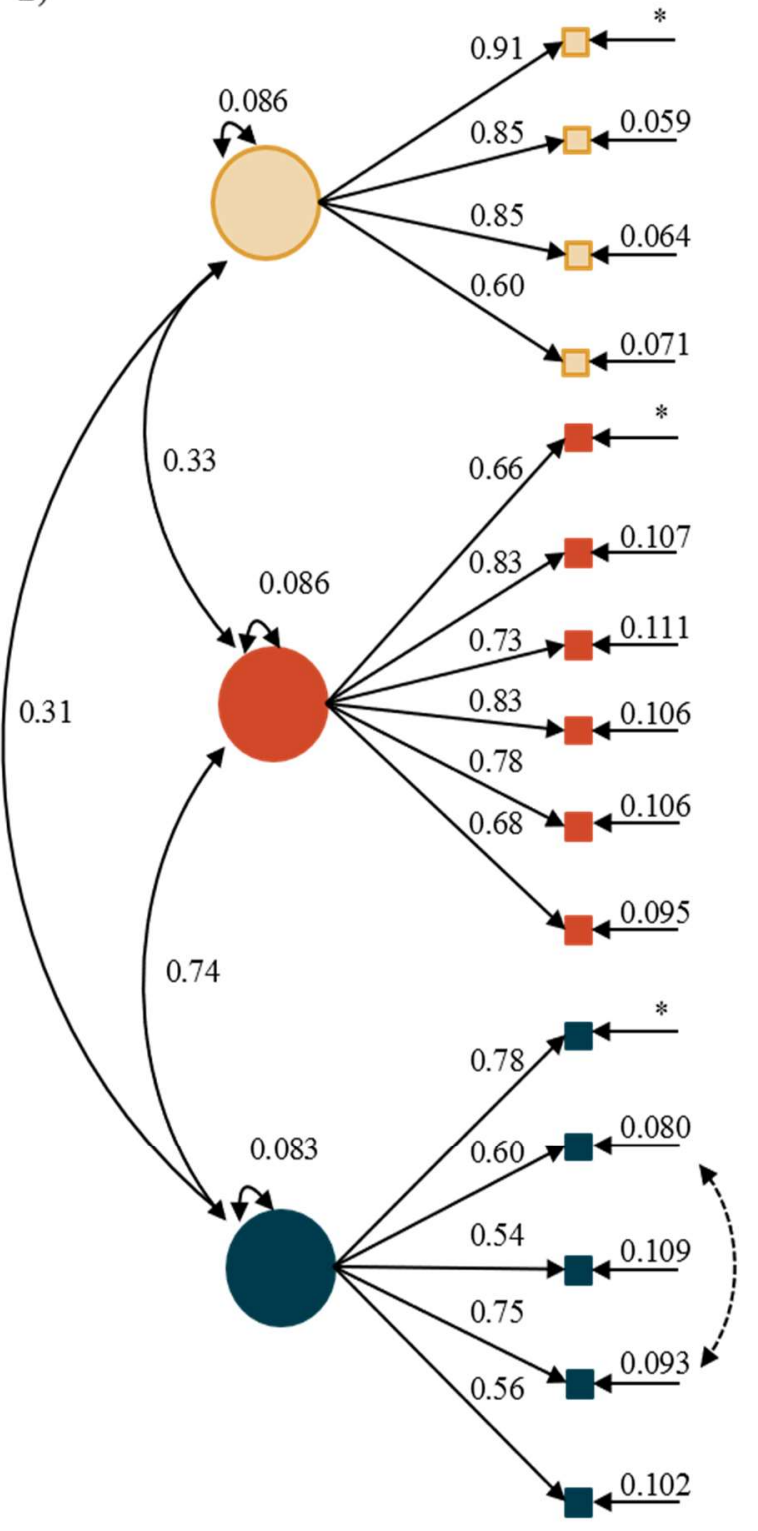

Figure B.4. Localized estimates for time 2, science worded, three-factor correlated models within A) general and B) organic chemistry. Dashed lines indicate nonsignificant paths. *Indicates the reference variable for the model. 